\title{
$\not 19.38$
}

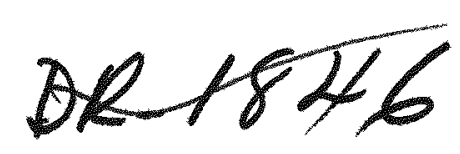

Wingint

\section{PUBLIC SAFETY}

$\&$

\section{UNDERGROUND}

NUCLEAR

DETONATIONS 


\section{DISCLAIMER}

This report was prepared as an account of work sponsored by an agency of the United States Government. Neither the United States Government nor any agency Thereof, nor any of their employees, makes any warranty, express or implied, or assumes any legal liability or responsibility for the accuracy, completeness, or usefulness of any information, apparatus, product, or process disclosed, or represents that its use would not infringe privately owned rights. Reference herein to any specific commercial product, process, or service by trade name, trademark, manufacturer, or otherwise does not necessarily constitute or imply its endorsement, recommendation, or favoring by the United States Government or any agency thereof. The views and opinions of authors expressed herein do not necessarily state or reflect those of the United States Government or any agency thereof. 


\section{DISCLAIMER}

Portions of this document may be illegible in electronic image products. Images are produced from the best available original document. 
TID-25708

\title{
PUBLIC SAFETY \& \\ UNDERGROUND \\ NUCLEAR DETONATIONS
}

\author{
by \\ Samuel Glasstone
}

June 1971

\begin{abstract}
This report was prepared as an account of work sponsored by the United States Government. Neither the United States nor the United States Atomic Energy Commission, nor any of thet employees, nor any of their contractors, subcontractors, or their employees, makes any warranty. express or implied, or assumes any legal liability or responsibility for the accuracy, completeness or usefulness of any information, apparatus, product or process disclosed, or represents that its use would not infringe pryately owned rights.
\end{abstract}

Published by the

UNITED STATES ATOMIC ENERGY COMMISSION 
Available as TID-25708 for $\$ 3.00$ from

National Technical Information Service

U.S. Department of Commerce

Springfield, Virginia 22151

Printed in the United States of America

USAEC Division of Technical Information Extension, Oak Ridge, Tennessee June 1971 


\section{FOREWORD}

This book is published in the hope that it will help to increase public knowledge and understanding of the extensive public safety program associated with underground nuclear testing, both in the Plowshare Program for peaceful applications of nuclear explosives, and in the vitally necessary weapons development program.

The book has been prepared through the coordinated effort of government agencies and contractors and is published by the U.S. Atomic Energy Commission. The Commission was fortunate in securing the services of Dr. Samuel Glasstone to author the book. Dr. Glasstone has the unique ability to present a highly technical and complex subject with clarity and in terms understandable to most of us.

The information in this book is as complete as possible within the limits of national security. The opinions of many competent U.S. scientists in a number of related disciplines are represented in the text.

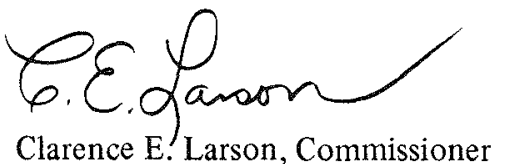

United States Atomic Energy Commission 
. 


\section{PREFACE}

The United States' program for testing nuclear explosive systems has two main objectives: first, to maintain the nation's security, and second, to develop peaceful applications of nuclear explosives for the benefit of all mankind. These objectives have been expressed in the Atomic Energy Act, passed by Congress in 1946 and amended in 1954, as follows: "The development, use, and control of atomic energy shall be directed so as to make the maximum contribution to the general welfare, subject at all times to the paramount objective of making the maximum contribution to the common defense and security." Among other things, the Act authorizes the U.S. Atomic Energy Commission to "conduct experiments and do research and development work in the military application of atomic energy." Such a research and development program requires the testing of new nuclear designs and concepts.

Furthermore, in 1963, the U.S. Senate gave its advice and consent to ratification of the treaty "banning nuclear weapons tests in the atmosphere, in outer space, and underwater." In taking this action, the Senate insisted on assurances by the President that certain safeguards would be implemented under the joint responsibility of the Atomic Energy Commission and the Department of Defense. One of these assurances is "the conduct of comprehensive, aggressive, and continuing underground nuclear test programs designed to add to our knowledge and improve our weapons in all areas of significance."

The maintenance of an assured deterrent against nuclear attack is a recognized aspect of national policy. This does not, of course, preclude efforts to achieve a workable international agreement for the limitation and control of nuclear armaments. But until a satisfactory agreement is reached and so long as other nations have the capability of launching a nuclear attack, a strong deterrent is mandatory. The development and testing of nuclear explosive devices is thus essential to national security.

Since the detonation of the first atomic bombs in 1945, there has been much interest in peaceful applications of nuclear explosives. Conventional (chemical) explosives have been and are being used in the excavation of canals, in mining and quarrying, in stimulating the flow of petroleum, and in other ways. In some applications it appears that nuclear explosives might have advantages over conventional explosives. Consequently, in 1957 the Atomic Energy Commission 
established the Plowshare program to develop peaceful uses of nuclear explosives. Among such possible uses are the excavation of canals and harbors, the fracturing of deep underground rocks to facilitate the flow of gas or oil, the breakup of ore bodies to permit extraction of minerals, the formation of large underground cavities for the storage of natural gas, liquid petroleum gas and oil, or liquid wastes, and the recovery of natural geothermal energy by cracking high-temperature rocks and utilizing their heat to produce steam for electrical power generation.

For the Plowshare program it is necessary to conduct underground nuclear detonations of two general types. The first is to develop and test nuclear explosives that are especially designed for their intended peaceful application; and the second is to perform actual experiments in the field to determine the most effective ways in which nuclear explosives can be used for specific peaceful applications. Both of these types of detonations are required if progress is to be made in the Plowshare program.

Nuclear explosions, like any other explosions, have the potential for some degree of risk to the public. By taking appropriate precautions, however, the risks can be reduced so that they are insignificant, especially in view of the importance of nuclear testing to the nation's security and economic progress. The purpose of this book is to describe and explain the Atomic Energy Commission's comprehensive safety programs for protecting the public from the effects of underground nuclear detonations. Experiments related to earthexcavation applications involve certain special aspects of public safety. Consequently, the specific safety procedures for these experiments, of which there have been only six in the Plowshare program, are treated in an Appendix.

In this book it will be shown that those responsible for ensuring safety in underground nuclear detonations have made, and will continue to make, every effort to implement their basic philosophy which has been summarized as follows: A nuclear device can be detonated safely only when it is ascertained that the operation can be accomplished without injury to people and domestic animals, directly or indirectly, and without an unacceptable damage risk to ecological systems (i.e., the balance of living things and their environment) and to natural and man-made structures.

I wish to take this opportunity to thank the members of the staff of the U.S. AEC Nevada Operations Office, in particular Robert E. Miller, Manager; Charles E. Williams, Deputy Manager; William R. Cooper, Assistant Manager for Plans and Budgets; William E. Gries, Director, Classification and Technical Information Division; and Robert R. Loux, Chief, Technical Information Branch, for their help and encouragement in writing this book.

Samuel Glasstone 


\section{ACKNOWLEDGMENT}

The following organizations cooperated in the production of this book by providing information and/or reviewing the draft manuscript:

U. S. Atomic Energy Commission Headquarters, Washington, D. C.

Division of Biology and Medicine

Division of Military Application

Division of Operational Safety

Division of Peaceful Nuclear Explosives

Division of Public Information

Division of Technical Information

U. S. Atomic Energy Commission Laboratories

Lawrence Radiation Laboratory, Livermore, University of California

Los Alamos Scientific Laboratory, University of California

Sandia Laboratories, Albuquerque

U. S. AEC Nevada Operations Office

Manager's Office

Test Manager

Assistant Manager for Administration

Assistant Manager for Engineering and Logistics

Assistant Manager for Operations

Assistant Manager for Plans and Budgets

Office of Chief Counsel

Office of Effects Evaluation

Office of Peaceful Nuclear Explosives

Office of Public Affairs

Classification and Technical Information Division

Radiological Operations Division

U.S. AEC Nevada Operations Office Contractors

Battelle Memorial Institute

Environmental Protection Agency, Southwestern Radiological Health Laboratory

Environmental Research Corporation

John A. Blume and Associates, Research Division 
National Oceanic and Atmospheric Administration, Air Resources Laboratory, Las Vegas, Nevada

National Oceanic and Atmospheric Administration, National Ocean Survey, Special Projects Party, Las Vegas, Nevada

Teledyne Isotopes

U. S. Geological Survey, Denver

The U.S. AEC Division of Technical Information Extension, Oak Ridge, prepared the line drawings for the illustrations and provided substantial assistance in compiling the Glossary. 


\section{CONTENTS}

Chapter 1 INTRODUCTION . . . . . . . . . . . . . . . . 1

Chapter 2 BASIC PRINCIPLES OF NUCLEAR EXPLOSIONS . . . . . 9

Chapter 3 UNDERGROUND NUCLEAR EXPLOSIONS: LOCAL EVENTS . . . . . . . . . . . . . . 33

Chapter 4 GEOLOGY, HYDROLOGY, AND SAFETY OF WATER SUPPLIES . . . . . . . . . . . . . . . 59

Chapter 5 GROUND MOTION AND THE RESPONSE OF STRUCTURES . . . . . . . . . . . . 82

Chapter 6 SEISMOLOGY AND WATER WAVE PHENOMENA $\quad \cdot \quad \cdot 121$

Chapter 7 METEOROLOGY AND RADIATION PREDICTIONS . . . 137

Chapter 8 THE RADIOLOGICAL SAFETY PROGRAM $\quad$. . . . . . 154

Chapter 9 THE BIOENVIRONMENTAL SAFETY PROGRAM . . . . 188

Chapter 10 SAFETY CONTROLS FOR NUCLEAR DETONATIONS . . 213 Appendix NUCLEAR EXCAVATION SAFETY . . . . . . . 228 REFERENCES .. . . . . . . . . . . . . . . 253 GLOSSARY . . . . . . . . . . . . . 255 INDEX . . . . . . . . . . . . . . . 266 


\section{Chapter 1}

\section{INTRODUCTION}

\section{TESTING OF NUCLEAR DEVICES}

For the reasons given in the Preface to this book, the United States is committed to a program of testing nuclear explosive devices for both peaceful and military purposes. In recent years, all these tests have been conducted underground, the only environment possible in order to comply with the limited test ban treaty signed by the United States in 1963. The treaty requires that no nuclear explosion be carried out "(a) in the atmosphere; beyond its limits, including outer space; or underwater, including territorial waters or high seas; or (b) in any other environment if such explosion causes radioactive debris to be present outside the territorial limits of the State under whose jurisdiction or control such explosion is conducted."

Most United States' underground nuclear tests have been conducted at the Nevada Test Site which at its closest point is about 65 miles northwest of Las Vegas, Nevada (Figs. 1.1 and 1.2). This will remain as the primary site for defense-related testing, but development of the Plowshare program for the peaceful uses of nuclear explosives would mean an increasing number of underground detonations in other areas.

As of the end of 1970 , there have been only three Plowshare tests outside the Nevada Test Site. The first such test was a scientific experiment near Carlsbad, New Mexico, in 1961. This was followed by joint U.S. Atomic Energy Commission and industry sponsored experiments for stimulating the flow of natural gas near Farmington, New Mexico, in 1967, and near Grand Valley, Colorado, in 1969. 


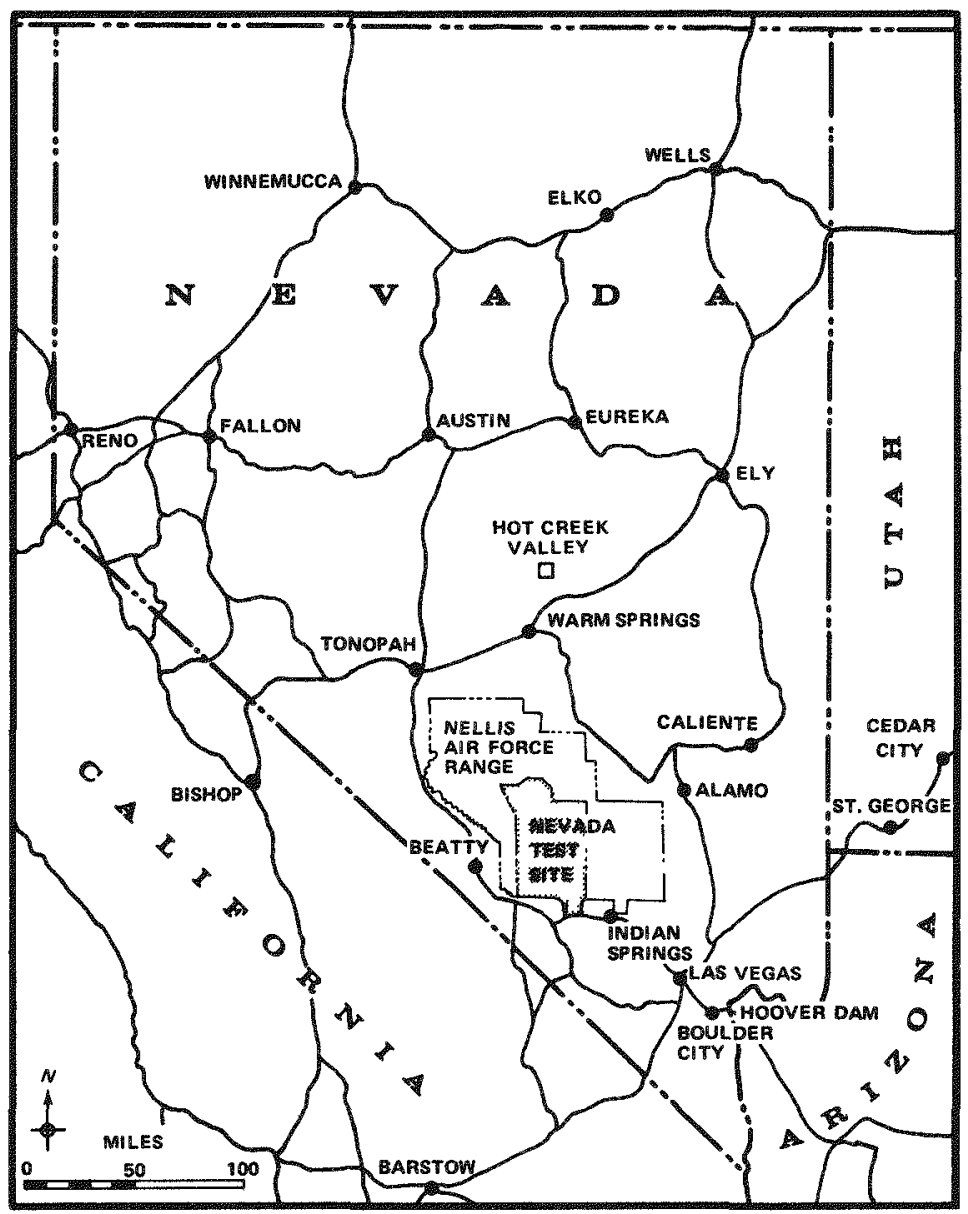

Fig. 1.1 Map showing locations of the Nevada Test Site and Hot Creek Valley, Central Nevada.

There have also been six other underground nuclear detonations elsewhere than the Nevada Test Site. Four of these (near Fallon, Nevada, in 1963; near Hattiesburg, Mississippi, in 1964 and 1966; and at Amchitka Island, Alaska, in 1965) were experiments in a Department of Defense program for improving means of detecting, locating, and identifying underground nuclear explosions. The other two were "calibration" tests at Hot Creek Valley, Central Nevada (see Fig. 1.1), in 1968, and at Amchitka Island, Alaska, in 1969. In a calibration test, a nuclear device of established energy release (or 


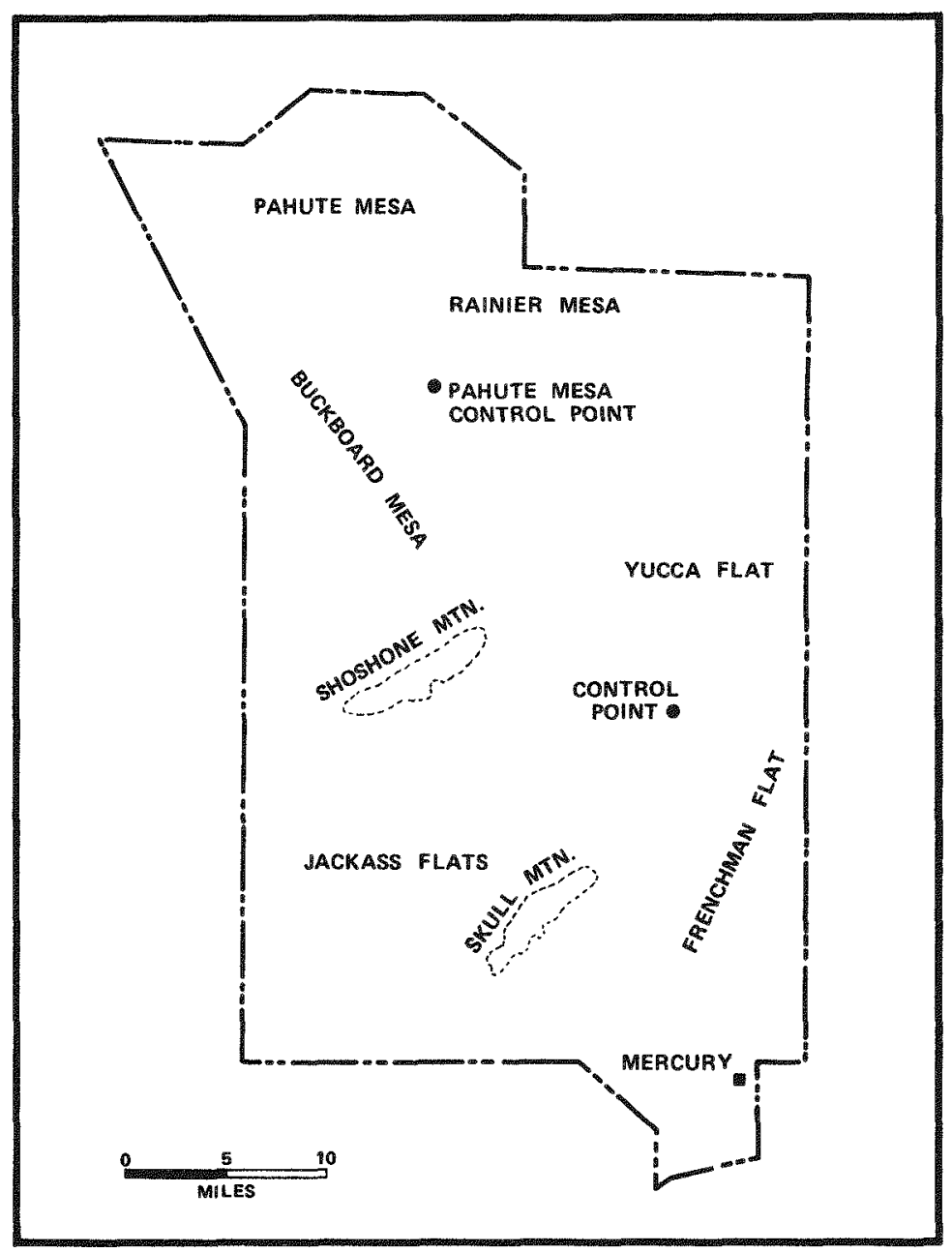

Fig. 1.2 Salient features of the Nevada Test Site.

yield) is detonated in a new area in order to determine the response of that area to an underground explosion.

The calibration tests were dictated by a need to develop supplemental sites for a limited number of underground tests of higher energy yields than are feasible at the Nevada Test Site. The limitation at this Site is the possible effect of the expected ground motion from detonations of large yield on off-site structures, especially high-rise buildings in Las Vegas. The test at Hot Creek Valley indicated that the area was suitable for events of higher yield, 
but an upper limit was set by the proximity of Las Vegas (175 miles) and Reno (200 miles). The Central Nevada Supplemental Area, as it is called, is presently held on a standby basis.

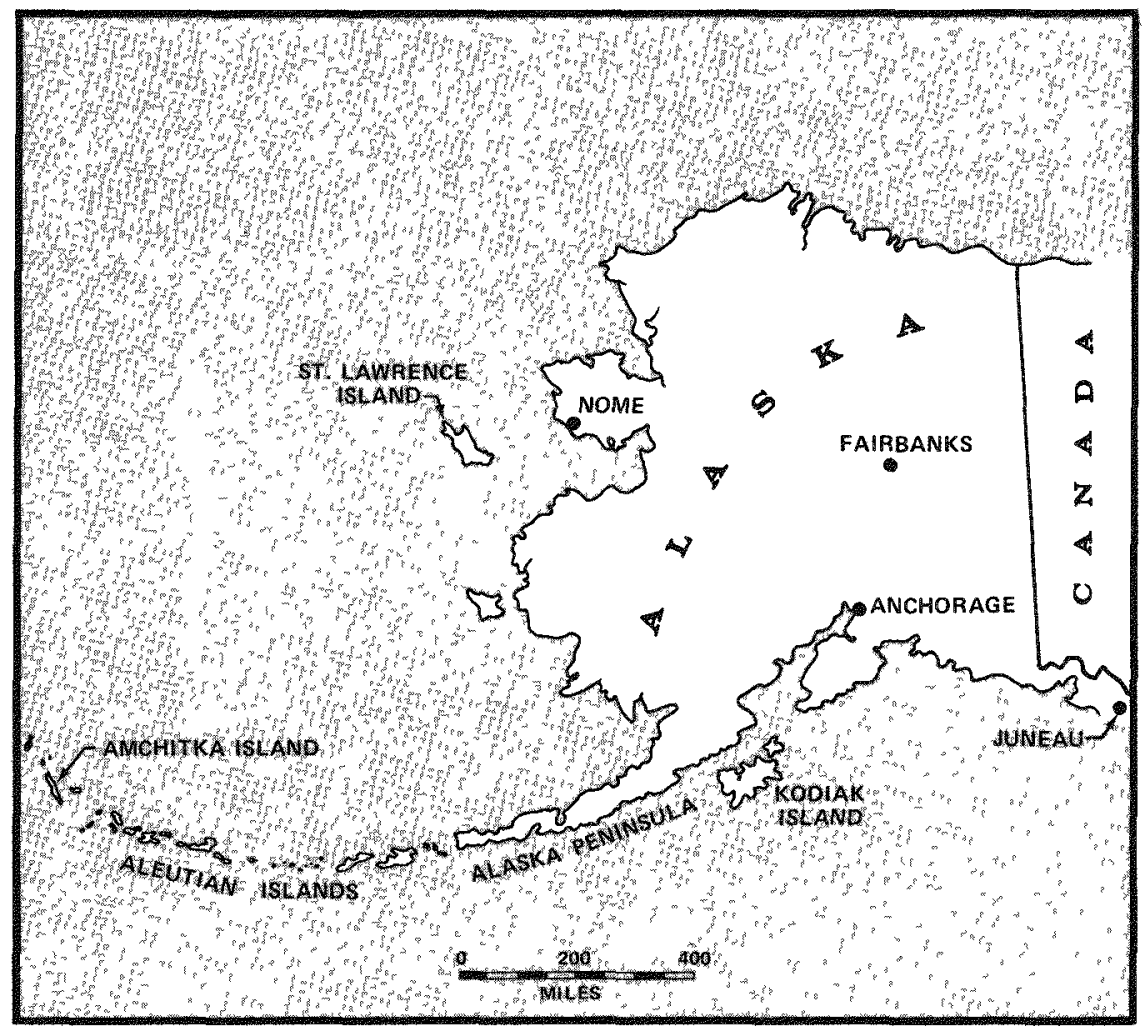

Fig. 1.3 Alaska and the Aleutian Islands showing location of Amchitka Island.

The calibration test on Amchitka Island showed that this island, near the western end of the Aleutian Islands chain (Fig. 1.3), could be used safely for the largest nuclear tests now envisioned. The nearest populated area is the Adak Naval Base about 200 miles distant, and the closest sizable communities are at least 1000 miles away. The Amchitka Island site is being prepared for the first underground weapons test in this area, probably in the autumn of 1971. 


\section{POTENTIAL HAZARDS IN NUCLEAR DETONATIONS}

Unless proper control is exercised, there could be harmful effects associated with any explosion. In an underground nuclear detonation such effects could arise mainly from the extremely high pressure generated in the ground by the explosion and from the radioactive residues of the nuclear device. These aspects of nuclear explosions will be considered more fully in later chapters.

For the present, however, it may be noted that the high pressure generates a shock wave which causes the ground to move, somewhat like an earthquake. If this motion were sufficiently strong, it could cause damage to man-made structures at considerable distances. In planning a nuclear test in Nevada or elsewhere, steps are taken to ensure that the effects of ground motion are minimal.

The great majority of underground nuclear detonations are performed at such depths that essentially all the radioactive residues are trapped in the ground. But if a nuclear device is exploded underground for an earth-excavation project, there is some release of radioactivity to the atmosphere. However, the operation would be conducted only when the conditions are such that there would be no harm to people and to animals, and to the environment outside the area disturbed by the explosion. If the purpose of an underground detonation in the Plowshare program is to recover gas, oil, or other commercial products, all possible precautions will be taken to assure that there is no radiation danger associated with any products that may eventually reach the public through normal supply channels.

\section{SAFETY PROGRAMS FOR NUCLEAR TESTS}

The directive of the U.S. Atomic Energy Commission to those responsible for conducting underground nuclear tests requires them to "take every precaution necessary, including delay or postponement of any detonation, to render negligible the hazards, both to the public and to on-site personnel, from any nuclear detonation." Similar considerations apply to structures of all kinds and to the environment. Consequently, in planning a nuclear explosion, regardless of its purpose, appropriate steps are taken to make sure that the test can be conducted without causing any injury to people and without significant damage to natural and man-made structures. 
Efforts are also made to prevent anything more than a minimum disturbance to animals and plants and their relationships to the local environment. The experience gained over a period of some fourteen years with more than three hundred announced underground nuclear detonations shows that these objectives have been met. The purpose of this book is to explain how this successful record has been achieved and how it will be maintained in the future.

Two separate but related programs have been established by the U.S. Atomic Energy Commission to make certain that all nuclear detonations, no matter where they take place, are conducted with safety to people, to structures, and to the environment. The first program is concerned with the safety measures adopted in preparation for each specific underground detonation project. This involves prediction of all conceivable effects and the development of detailed plans to prevent or mitigate those which are potentially harmful. These plans are carefully reviewed at all stages to ensure their adequacy and reliability.

The second aspect of the safety program involves long-range studies for continuously improving the understanding of the complex phenomena of underground nuclear detonations. These studies have resulted in better predictions of the effects and consequently in better safety precautions. Furthermore, the information obtained can be used to develop plans for new programs and for achieving safe explosions at new locations.

The two parts of the overall safety program are dependent upon each other. On the one hand, the long-range analyses utilize the many measurements made before, during, and after each underground explosion. The safety procedures associated with a specific detonation, on the other hand, benefit from the improved prediction capabilities that result from the long-range studies.

\section{OUTLINE OF THIS BOOK}

As a first step in understanding the overall safety problem, something is said in Chapter 2 of this book about those aspects of the atom that have a direct bearing on nuclear explosions and their effects. The two ways in which atomic (or nuclear) energy can be released in explosive form, namely by fission and fusion, are explained. 
In Chapter 3, the events in the immediate vicinity of an underground nuclear explosion are described. These events are the sources of the two potential hazards mentioned above-ground motion and radioactivity. It is shown that the escape of radioactivity can be prevented if the nuclear device is properly buried at a sufficient depth and major fractures in the ground are avoided.

The importance of the geology and hydrology of the explosion region is dealt with in Chapter 4 . The geology of the area has a bearing on the propagation of the shock wave generated by the explosion. Furthermore, studies in geology and hydrology, which indicate the presence and movement of water, are used to ensure that no significant amount of radioactivity will ever appear at a point where water is consumed by people or animals.

The motion of the ground at a distance from the underground nuclear detonation and the response of structures to this motion are examined in Chapter 5. It is shown how the responses of buildings and the probability of damage, if any, are predicted in advance of an actual explosion.

In Chapter 6 consideration is given to another aspect of ground motion, namely, the minor earth tremors (or aftershocks) that may continue for a time after an underground nuclear detonation. The aftershocks, which so far have always been of smaller magnitude than the initial seismic effect of the explosion, appear to result from the release of pre-existing strains in the ground. The possibility of generating large waves in the ocean or in enclosed bodies of water by an underground detonation is also examined in Chapter 6.

The extensive meteorological program associated with underground nuclear testing is outlined in Chapter 7 . The main objective of the program is to determine if the weather conditions are suitable for conducting the detonation, bearing in mind the possibility that some radioactivity might be accidentally released to the atmosphere.

The accidental release of radioactivity as the result of an underground nuclear detonation at the Nevada Test Site is relatively rare. If it does occur, the activity does not usually extend beyond the boundaries of the Test Site. Nevertheless, prior to each event, an extensive radiation protection program is established and it is continued as long as necessary after the detonation. This program is described in Chapter 8.

The bioenvironmental safety program is discussed in Chapter 9. The concern here is with the possible indirect and long-range 
consequences of the effects of underground nuclear explosions on all forms of life and their environment. In conducting a nuclear test, efforts are made to keep ecological disturbances to a minimum.

Finally, the extensive planning in advance of every underground nuclear explosion to ensure safety is reviewed in Chapter 10. It is only after careful evaluation of the conditions and possible effects of each such detonation by many experienced individuals that the event is authorized.

The treatment in Chapters 3 through 8 does not include the effects of experiments in the use of nuclear explosives for large-scale earth excavation. Since these experiments have represented a very small fraction of the total number of underground detonations and involve special precautions, they are discussed in an Appendix. 


\section{Chapter 2 \\ BASIC PRINCIPLES \\ OF NUCLEAR EXPLOSIONS}

\section{CHARACTERISTICS OF ATOMS}

\section{Elements and Atoms}

All naturally occurring substances on earth are made up from one or more of about 90 different kinds of simple materials known as "elements." Among the common elements are the gases, oxygen and nitrogen, which are the main components of air; solid nonmetals, such as carbon and sulfur; and various metals, including iron, zinc, and copper. There are also other elements, for example, mercury, that are normally liquids. A less familiar element, which has attained prominence in recent years because of its use as a source of nuclear energy, is the metal uranium.

The smallest part of any element that can exist, while still retaining the characteristic properties of the element, is called an "atom" of that element. Thus, there are atoms of hydrogen, of iron, of uranium, and so on, for all the elements. The hydrogen atom is the lightest of all atoms, whereas the atoms of uranium are the heaviest atoms found in nature. Still heavier atoms, such as those of plutonium, also important for the release of nuclear energy, have been made artificially starting with uranium.

\section{The Nucleus of the Atom}

Even the heaviest (and largest) atoms are extremely small. About 100 million of the larger atoms placed side by side would extend for 
a length of one inch. In spite of their small size, atoms have an internal structure. Every atom consists of an even smaller central region or "nucleus" surrounded by light particles called "electrons" (Fig. 2.1). The nucleus carries a positive charge of electricity, whereas the electrons have a negative charge. In the normal atom, the

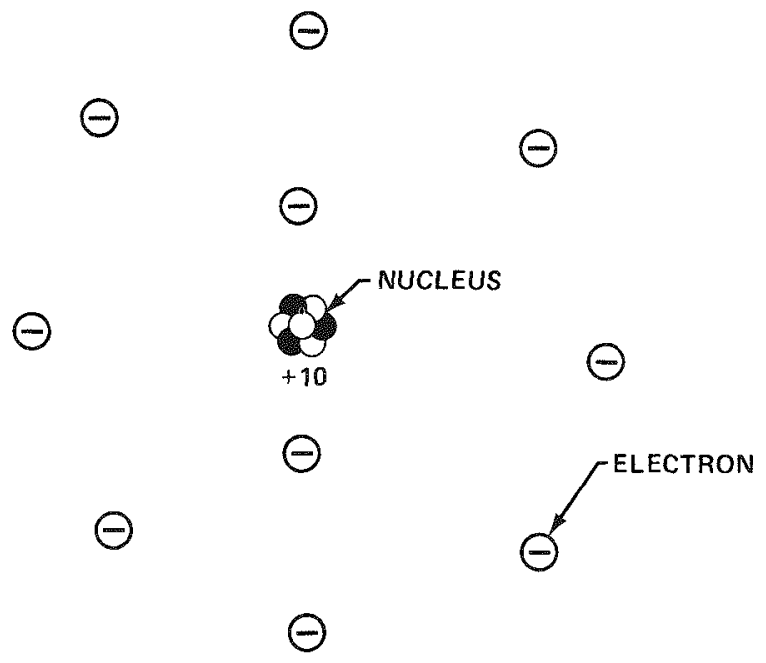

Fig. 2.1 Representation of the structure of an atom (not to scale). This particular atom has ten positive charges (protons) in the nucleus which is surrounded by ten electrons.

positive charges on the nucleus are exactly balanced by the negative charges of the electrons; the atom as a whole then has no net electrical charge. In other words, the atom is said to be electrically neutral.

It is possible to go a stage further and note that the atomic nucleus is itself made up of even simpler fundamental particles, referred to as "protons" and "neutrons." The nucleus of the light hydrogen atom is merely a proton, but the nuclei of all other atoms contain both protons and neutrons. These two particles have almost the same mass, but they differ in the following respect: the proton carries a single charge of positive electricity, whereas the neutron, as its name implies, carries no charge and is electrically neutral. It is, in fact, the protons that are responsible for the positive charge on the nucleus. However, both the protons and the neutrons contribute to the mass of the nucleus. Because the electrons have such a very small 
mass, the nucleus carries nearly all-99.94 to 99.98 percent of an atom's mass.

\section{The Existence of Isotopes}

The essential difference between atoms of different elements lies in the number of protons (or positive charges) in the nucleus; this is called the "atomic number" of the element. The nuclei of hydrogen atoms, as stated above, each contain one proton; helium atoms have two protons, uranium atoms have 92 protons, and plutonium atoms contain 94 protons. Although all the nuclei of a given element have the same number of protons, they may have different numbers of neutrons. The resulting atomic species, which have identical atomic numbers but which differ in their masses (i.e., total number of protons and neutrons), are called "isotopes" of the particular element.

There are two naturally occurring elements, in particular, whose isotopes are of special interest for the realization of nuclear explosions, namely, hydrogen and uranium. The element hydrogen exists in the form of three isotopes; they are called hydrogen (or
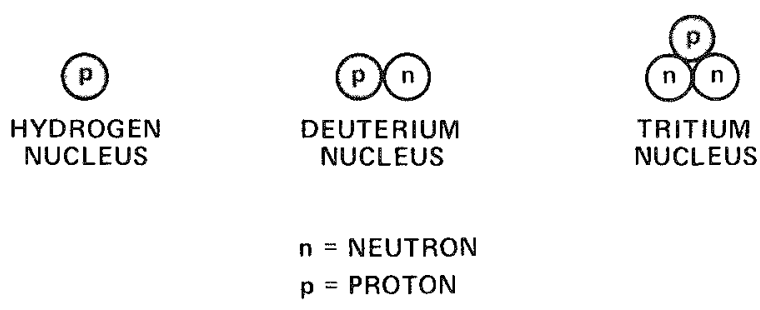

Fig. 2.2 Nuclei of the three isotopes of hydrogen.

light hydrogen), deuterium (or heavy hydrogen), and tritium, respectively. The compositions (Fig. 2.2) and masses of the nuclei of these three isotopes are given below. The mass or "mass number" is the sum of the numbers of protons and neutrons in each case.

\begin{tabular}{cccc}
\hline & Hydrogen & Deuterium & Tritium \\
\hline No. of protons & 1 & 1 & 1 \\
No. of neutrons & 0 & 1 & $\frac{2}{3}$ \\
Mass number & $\overline{1}$ & $\overline{2}$ & $\overline{3}$ \\
\hline
\end{tabular}


Uranium as found in nature consists mainly of two isotopes; they have mass numbers of 235 and 238 and are designated uranium-235 and uranium-238, respectively. In each case, the atomic nucleus contains 92 protons with the remainder of the mass made up by neutrons (Fig. 2.3). The lighter of these isotopes, uranium-235,
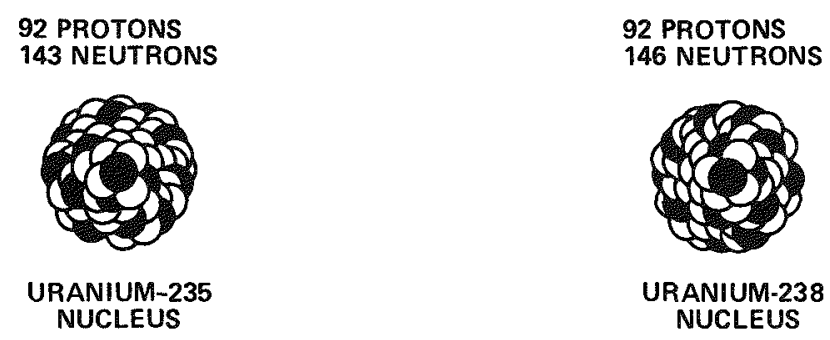

Fig. 2.3 Representations of the nuclei of uranium-235 and uranium-238.

which is by far the less abundant of the two, serves as the explosive material in many nuclear devices.

\section{Radioactive Isotopes and Their Radiations}

All but about twenty of the known elements exist in nature in two or more isotopic forms. Most of these natural isotopes are stable; that is to say, the nuclei do not change in any way over long periods of time. Some isotopes, however, have nuclei that are not stable; they continuously undergo changes by emitting radiations. These unstable isotopes are said to be "radioactive" and to exhibit the phenomenon of "radioactivity." The process of radioactive change is commonly referred to as "radioactive decay." About forty radioactive isotopes (or "radioisotopes") of twelve heavy elements, such as uranium and radium, occur naturally on earth. In addition, more than a thousand radioactive isotopes have been produced artificially by various nuclear reactions.

Three kinds of radiation are associated with the more common types of radioactive decay. First, there are "alpha particles," which consist of two protons and two neutrons, and are identical with the nuclei of helium atoms. Alpha particles from radioactive sources can travel no more than two or three inches in air (Fig. 2.4) before they pick up two electrons and thus become ordinary atoms of helium. In 
materials more dense than air, alpha particles cease to exist within much shorter distances. For example, they are unable to get through the outer layers of the human skin.

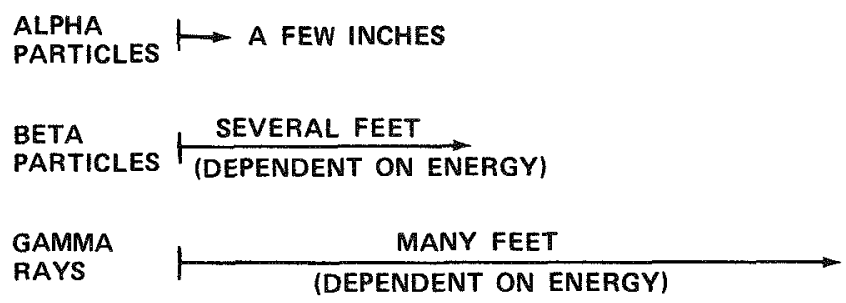

Fig. 2.4 Qualitative comparison of the penetrations of alpha and beta particles and gamma rays in air.

"Beta particles" represent the second type of radiation. These particles are actually electrons moving at very high speeds. However, they are not those electrons that normally surround the nucleus, as shown in Fig. 2.1. In the isotopes that emit beta particles, a neutron in the nucleus changes spontaneously into a proton and an electron. The proton remains, but the electron is expelled immediately as a beta particle (see Fig. 2.9). Beta particles can travel several feet through air before they are absorbed and they can penetrate the human skin to a depth of a very small fraction of an inch.

The nuclei of nearly all radioisotopes emit either an alpha particle or a beta particle. This emission is often accompanied by the third kind of nuclear radiation, called "gamma rays"; these are similar to $\mathrm{X}$ rays but generally have more energy. Gamma rays can travel great distances through air and can pass through appreciable thicknesses of denser material. Thus gamma rays are sometimes able to penetrate quite deeply into the body.

Exposure of living organisms to sufficiently large amounts of alpha or beta particles or gamma rays can have harmful effects. Because they travel only such short distances, alpha particles do not present any danger provided the source is outside the body. But if an appreciable quantity of a radioactive isotope gets into the body, through food, water, or air, the effects could be serious.* Thus, alpha

*It will be seen in Chapter 8 that the human body always contains significant amounts of radioisotopes of potassium and carbon derived from natural sources. These isotopes emit beta particles. 


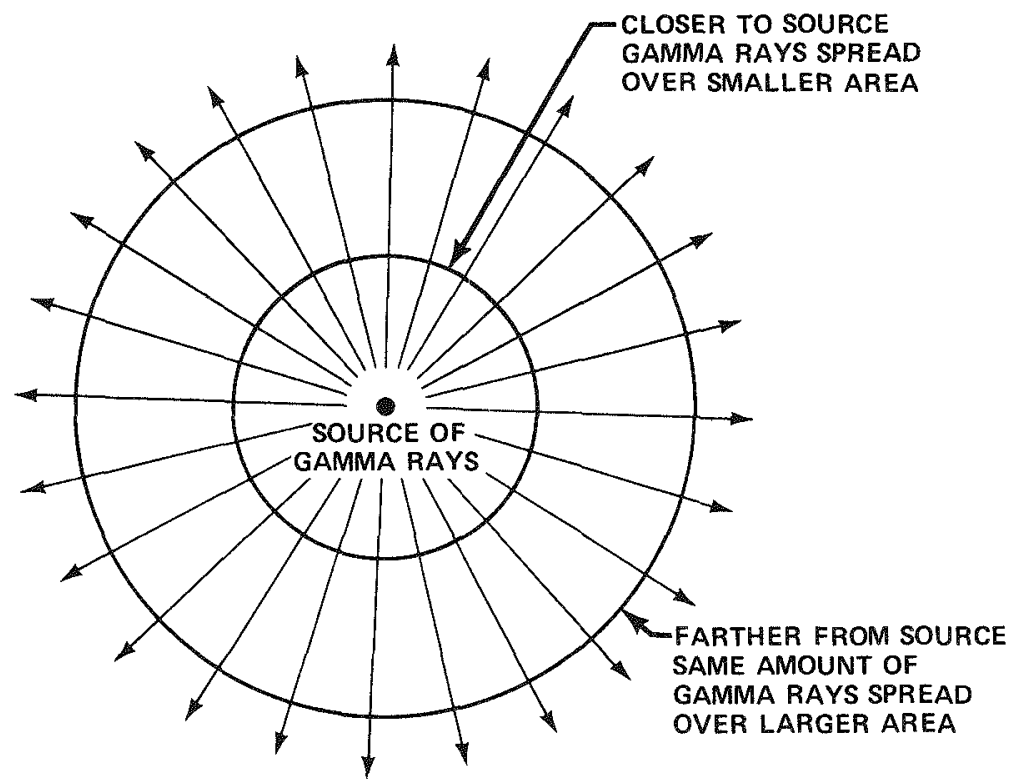

Fig. 2.5 The intensity of gamma rays decreases farther from the source.

particle emitters are largely a potential "internal" hazard. Sources of beta particles are also potential internal hazards, although if they remain in contact with the skin for a time they can cause severe burns.

Radioisotopes that emit gamma rays are important because they are potential "external" as well as internal hazards. These radiations can travel considerable distances in air and so they can cause injury even when they originate from sources at some distance from the human body. However, the farther the gamma rays travel, the smaller is their intensity (and hazard) from a given source (Fig. 2.5). Thus, the intensity at 20 feet is one-fourth and at 30 feet one-ninth of that at 10 feet from a point source.

\section{Radioactive Decay and Half-Life}

The emission of radiations from radioactive isotopes is a spontaneous process that is continuous and gradual. It takes place over a period of time at a rate depending on the nature of the radioisotope and upon the quantity present. Because of the continuous decay, the amount of the radioactive material and the rate of emission of radiation decrease steadily in the course of time. 
The rate of radioactive decay of a specified radioisotope, that is, the rate at which alpha or beta particles and gamma rays (if any) are emitted from its nuclei, is usually expressed in terms of a property known as the "half-life." This is defined as the time required for the radioactivity of a given amount of a particular isotope to decrease (or decay) to half its original value.

An important aspect of radioactive decay is that no matter how much of a given isotope is considered-it may be several pounds or a small fraction of an ounce-it always takes the same time for the activity (or rate of particle emission) to decrease to half of the initial value. The rate of decay of radioactivity in this manner is illustrated in Fig. 2.6.

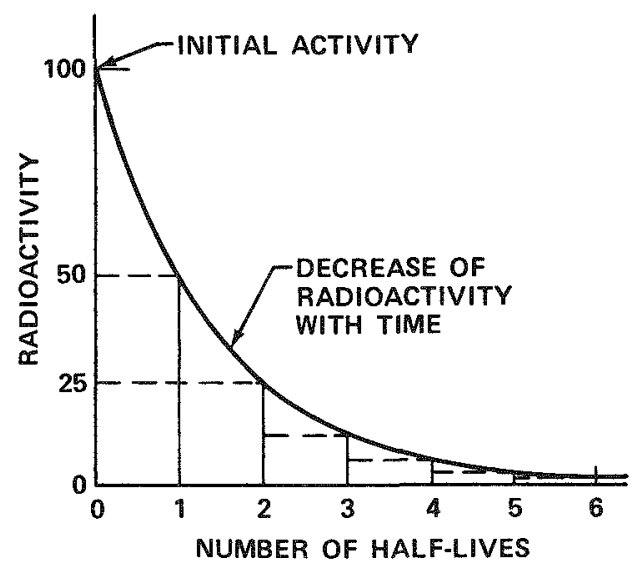

Fig. 2.6 Graphical representation of the decay of a radioactive isotope.

Suppose the activity of a certain amount of a radioactive material at any time is represented by 100 ; this corresponds to zero time on the curve. Then, after one half-life, the activity will be down to 50; another half-life later, it will have decreased to 25; after three half-lives, the activity will be down to 12.5 , and so on. Thus, it takes a little more than three half-lives for the activity of any quantity of radioactive material to decay to 10 percent of its initial value. In somewhat less than seven half-lives the activity will be down to 1 percent, and in ten half-lives it will be only 0.1 percent.

Each of the individual radioactive isotope species has a definite half-life which is a characteristic property of that isotope. These half-lives can be determined in the laboratory by means of instruments which measure how the rate of radioactive decay (or 
particle emission) changes with time. The half-life may be shorter than a millionth of a second or as long as billions of years, according to the nature of the isotope.

The half-lives of some radioisotopes of interest in connection with nuclear detonations are given in the accompanying table.

Half-Lives of Some Radioisotopes

\begin{tabular}{ll}
\multicolumn{1}{c}{ Isotope } & Half-Life \\
\hline Iodine-131 & 8 days \\
Krypton-85 & 10.8 years \\
Tritium & 12.3 years \\
Strontium-90 & 28 years \\
Cesium-137 & 30 years \\
\hline
\end{tabular}

As far as the potential hazard of radioactivity is concerned, radioisotopes of very short or very long half-life are usually not important. If the half-life is a matter of minutes or less, the activity will become insignificant within an hour or so. On the other hand, if the half-life is many thousands of years, the rate of particle emission is quite small unless the quantity of radioactive material is large. Special attention must therefore be paid to the isotopes with half-lives that are neither too short nor too long in relation to the human life span.

A radioactive substance of long half-life could be a hazard, however, if it entered the human body and remained there for a considerable time. Of special importance in this respect is the man-made radioisotope plutonium-239, which is used in some nuclear explosive devices. This isotope emits alpha particles and has a half-life of more than 24,000 years. If plutonium-239 gets into the body, it tends to collect in the bones, and because of its chemical nature and long half-life it remains there for many years continuously emitting alpha particles. Thus, plutonium-239 is a significant potential internal hazard.

\section{Radioactive Decay Products}

As the result of the expulsion of either an alpha particle or a beta particle, the nucleus of a radioactive substance is changed into the nucleus of another element, referred to as the "decay (or daughter) 
product." The nucleus remaining after alpha-particle emission contains two protons and two neutrons less than did the original ("parent") nucleus (Fig. 2.7). The atomic number (i.e., number of

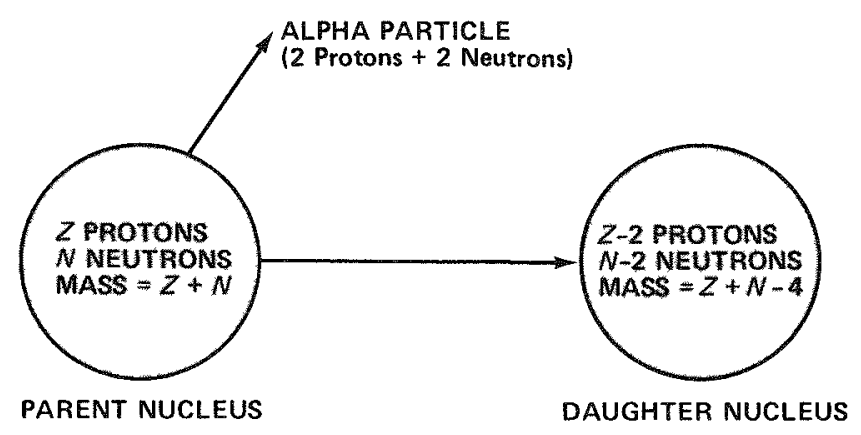

Fig. 2.7 Change in a nucleus by the emission of an alpha particle.

protons) of the daughter nucleus is thus two units less and its mass number (i.e., number of protons and neutrons) is four units less than the parent. In beta-particle emission, a neutron in the nucleus is changed into a proton, as stated earlier. Hence, the mass number of the daughter is the same as that of the parent, but the atomic number is one unit larger (Fig. 2.8). Gamma rays, however, are a form of energy and their emission leaves the numbers of neutrons and protons in the nucleus unchanged.

In some cases, the daughter nucleus produced by radioactive decay is stable, that is to say, it is not radioactive and does not decay further. In many instances, however, the daughter is also radioactive.

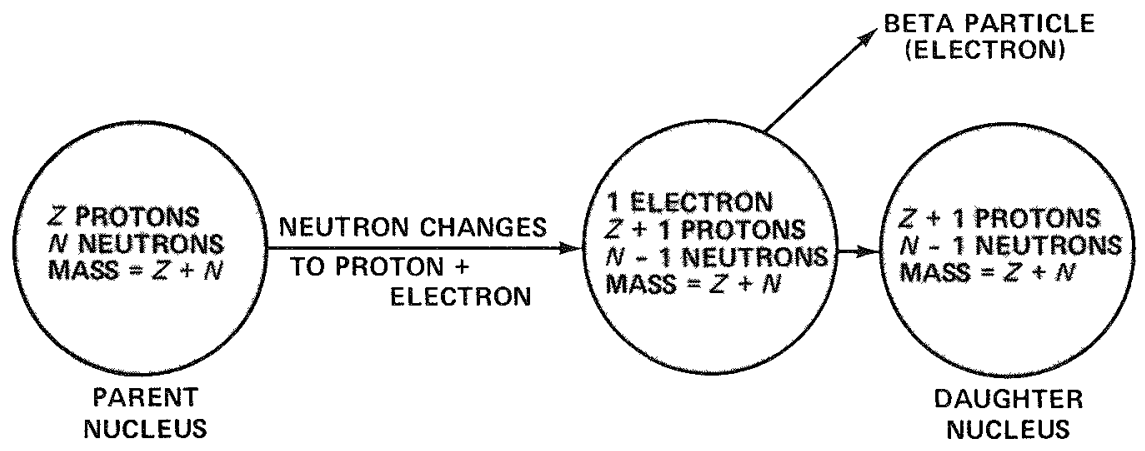

Fig. 2.8 Change in a nucleus by the emission of a beta particle. 
If so, it will decay with its own characteristic half-life, emitting either an alpha or a beta particle and possibly gamma rays. Eventually, after a series of radioactive changes, a stable isotope is formed (see Fig. 2.16).

\section{THE RELEASE OF NUCLEAR ENERGY IN EXPLOSIONS}

\section{Chemical and Nuclear Explosives}

Until the early 1940's, when means were found for releasing nuclear energy, the most important sources of energy depended on chemical reactions. This was true for explosives as well as for the production of electricity. Chemical high explosives are used extensively for peaceful purposes: in mining and quarrying, in earthexcavation projects, to stimulate flow in some gas and oil wells, and in other ways.

Almost 1400 tons of chemical explosive in a single charge were used, for example, to blow up Ripple Rock in British Columbia, Canada (Fig. 2.9). This rock, located in the Seymour Narrows, had previously been a hazard to shipping. It will be seen shortly that an equivalent explosive effect could have been produced with a very much smaller mass of a nuclear explosive.

The release of energy from conventional explosives and fuels, such as coal, oil, and gas, depends on chemical reactions or chemical changes. In such changes, atoms of different elements exchange partners; in other words, there are rearrangements among the atoms. These rearrangements involve only the electrons, with the nuclei of the atoms being unaffected. By contrast, the so-called atomic energy arises from rearrangements (i.e., splitting or combining) within the nuclei themselves. It is for this reason that the correct name is "nuclear energy."

The forces between the protons and neutrons in an atomic nucleus are millions of times as strong as those between electrons. As a result, a given mass of a nuclear fuel or explosive will produce several million times as much energy as the same mass of a conventional (chemical) fuel or explosive, such as TNT (Fig. 2.10). Nuclear energy may thus be thought of as a very "concentrated" form of energy.

In this book we are interested in nuclear explosions. It may be noted therefore that, in an explosive device, nuclear splitting (fission) 


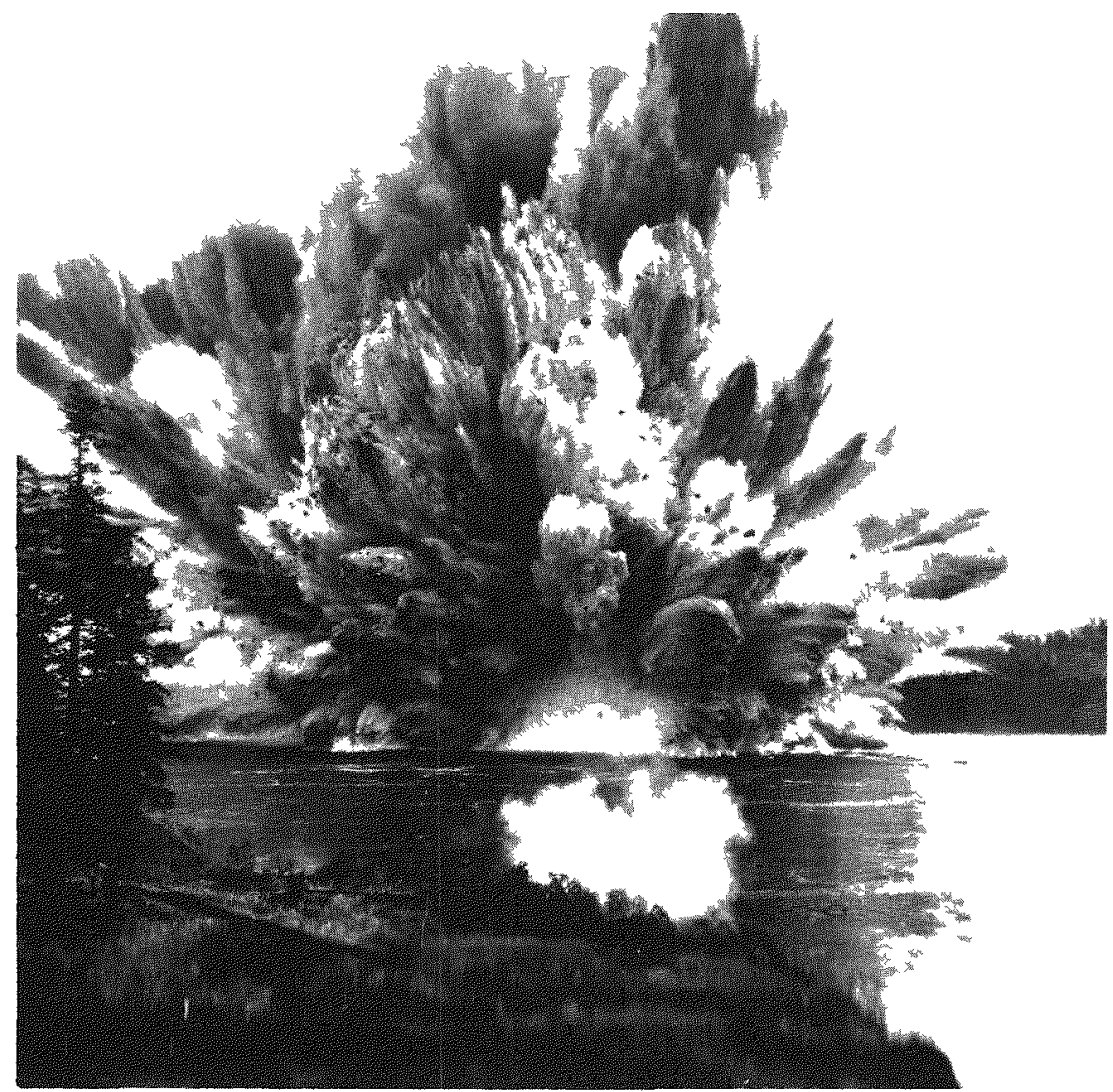

Fig. 2.9 Ripple Rock, in the Seymour Narrows, British Columbia, is destroyed by a single charge of 1400 tons of chemical high explosive.
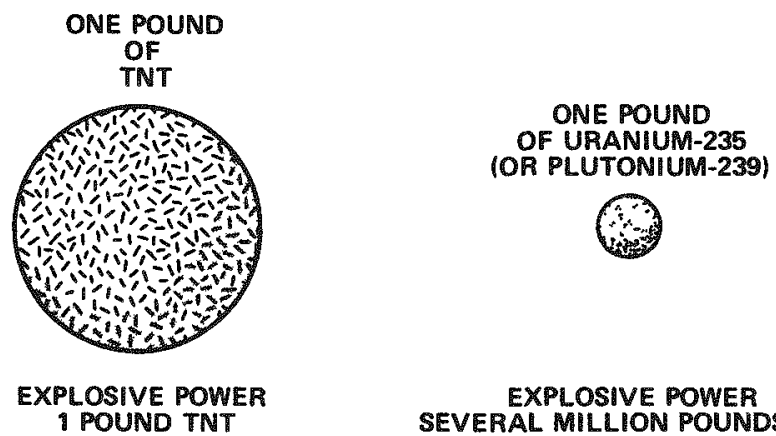

EXPLOSIVE POWER SEVERAL MILLION POUNDS TNT

Fig. 2.10 Comparison of explosive powers of equal weights of TNT and a nuclear explosive. 
of 1 pound of uranium-235 would produce as much energy as 16 million pounds or 8000 (short) tons of the chemical high explosive TNT. Not all of the uranium-235 nuclei present are "split" in a nuclear explosion. Nevertheless, a nuclear device is generally some millions of times more powerful than a chemical explosive of the same size or mass.

The "yield" of a nuclear explosive device is a measure of the amount of energy released when it explodes. It is the usual practice to state the yield in terms of the quantity of TNT that would produce the same amount of explosive energy. Thus, the yields are expressed as tons, thousands of tons (kilotons), or millions of tons (megatons) of TNT equivalent. Nuclear devices of low yield have TNT equivalents of a few kilotons or less, whereas those of high yield are equivalent in energy to about a megaton (i.e., a thousand kilotons) or more of TNT.* In most nuclear tests made by the United States, the yields have been in the range between a few kilotons and several hundred kilotons.

Two general methods are known for the explosive release of nuclear energy. They are called nuclear "fission" and "fusion," respectively. In fission, which means splitting, the nuclei of certain heavy atoms are made to break up into lighter particles. The total mass of all of the fragments formed is less than the mass before fission, and so there is a net loss of mass in the reaction. In nuclear fusion, on the other hand, a pair of very light nuclei combine (or fuse) together to form a nucleus of a heavier atom. Again there is a net loss of mass in that the products are lighter than the original nuclei. The conversion of this "lost" mass into energy accounts for the large amounts of energy released in both fission and fusion processes.

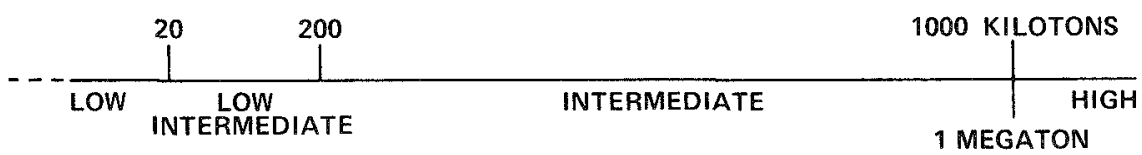

Fig. 2.11 Common convention for describing energy yields of nuclear explosions.

*According to a common convention, "low" yield means less than 20 kilotons; "low intermediate" means 20 to 200 kilotons; "intermediate" means 200 to 1000 kilotons (1 megaton); and "high yield" refers to more than 1 megaton (Fig. 2.11). 


\section{Energy from Nuclear Fission}

The main materials used in nuclear fission are uranium-235 and plutonium-239. They are called "fissile" isotopes. The former of these isotopes is present in natural uranium and is separated from the more abundant isotope, uranium-238. Plutonium-239, however, has not been found naturally on the earth; it is made from uranium-238 by certain nuclear reactions.

Although neutrons usually occur in atomic nuclei, it is possible to obtain free (or unattached) neutrons. When such a free neutron enters the nucleus of a fissile atom, it can cause the nucleus to split into two parts. This is the fission process, which is accompanied by the release of a large amount of energy. The smaller (or lighter) nuclei that are formed by the fission of the heavier nucleus are called "fission fragments."

The important point about the fission of a uranium-235 (or of a plutonium-239) nucleus by means of a neutron is that, in addition to the liberation of a large amount of energy, the process is accompanied by the instantaneous emission of two or three neutrons (Fig. 2.12). The fission reaction may thus be expressed as follows:

Neutron + uranium-235 $\rightarrow$ Fission fragments

+2 or 3 neutrons + energy

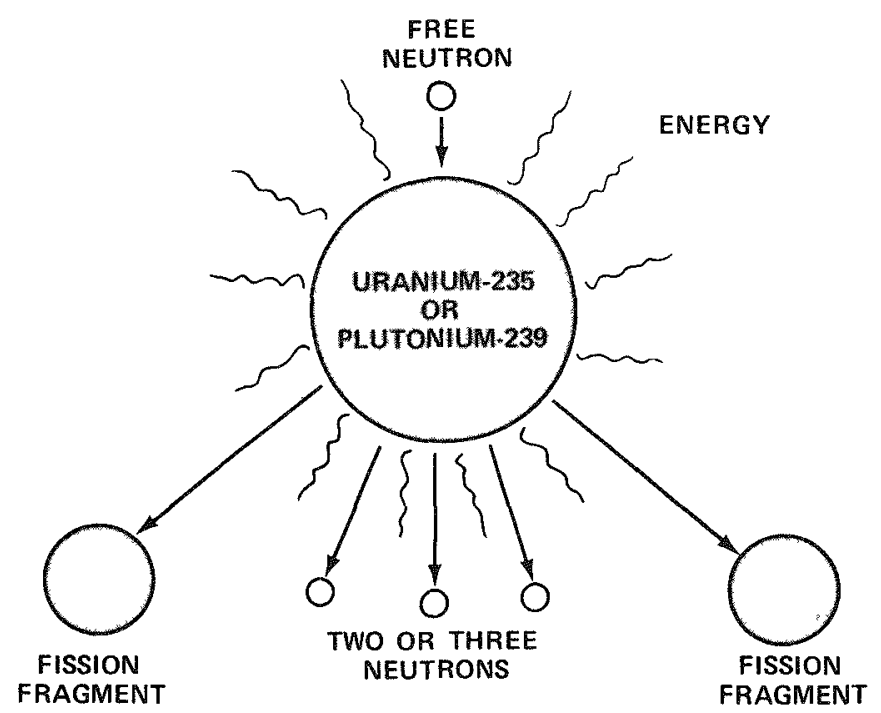

Fig. 2.12 The fission of a uranium-235 (or plutonium-239) nucleus by a neutron. 
The neutrons set free in the fission reaction are able to cause fission of additional uranium-235 (or plutonium-239) nuclei. Each such fission process is accompanied by the emission of more neutrons which can produce further fissions, and so on. Thus, in principle, a single neutron could start off a chain of nuclear fissions. In such a chain, as represented in Fig. 2.13, the number of nuclei undergoing fission, and the energy liberated, could increase very rapidly.

Although from two to three neutrons, on the average, are produced for each nucleus that undergoes fission, they are not all available for causing more fissions. The reason is that some of the neutrons escape altogether whereas others are removed in various nonfission reactions. Thus, in Fig. 2.13, one of the three neutrons shown as being released in the fission of a uranium-235 nucleus is indicated as "lost." But, for each act of fission, two neutrons are seen to be available to carry on the fission chain.

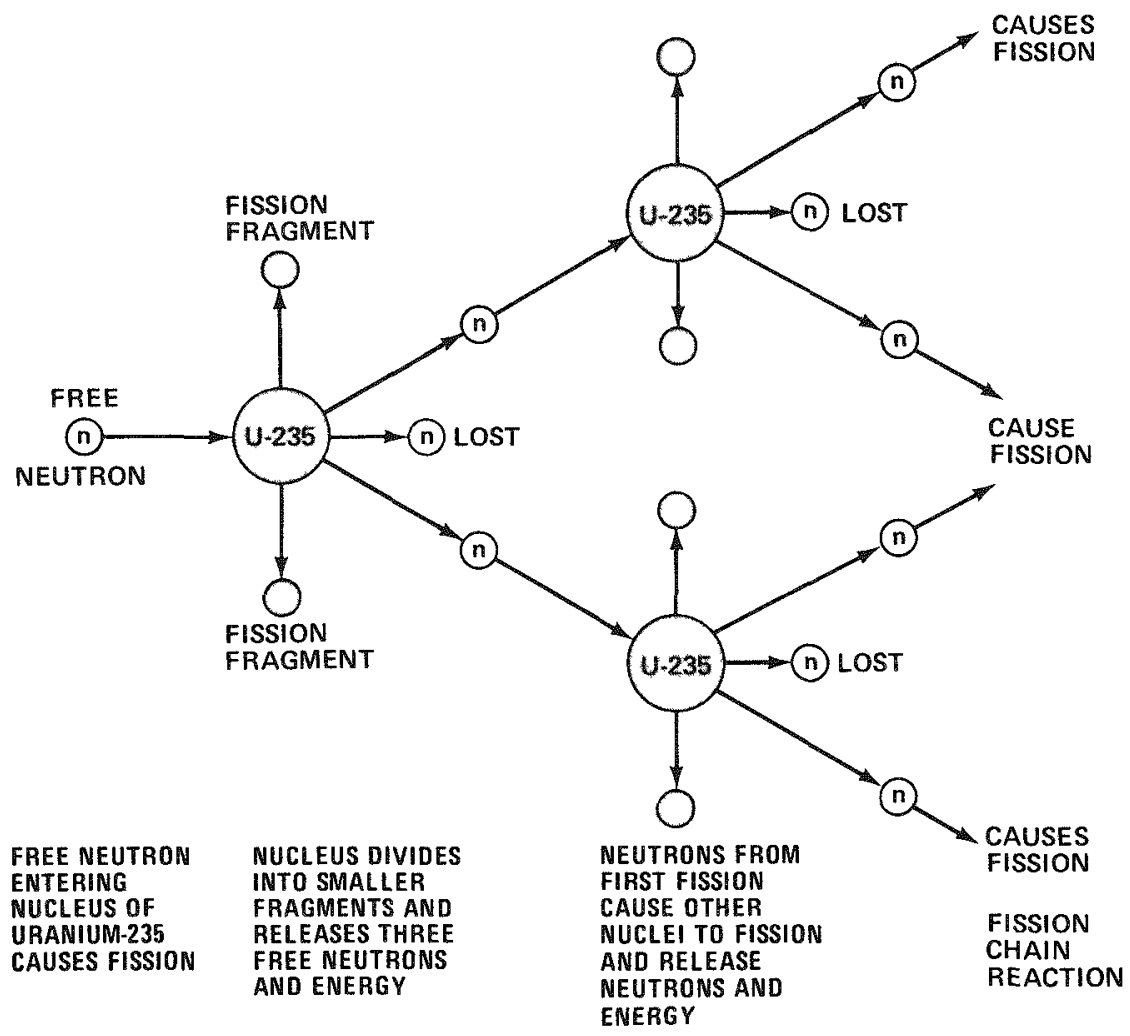

Fig. 2.13 Representation of a fission chain in a supercritical mass. 
In some circumstances, however, the loss of neutrons can be so great, relative to the number released by fission, that not enough neutrons are left to sustain the fission chain. Such an arrangement of fissile material is said to be "subcritical." In many subcritical systems, the loss of neutrons is due in large measure to their escape from the fissile material. If this loss by escape could be reduced, a self-sustaining chain reaction would be possible. The minimum quantity of a fissile isotope in which a self-sustaining fission chain can occur, under given conditions, is referred to as the "critical mass" for those conditions.

\section{Achieving a Fission Explosion}

In order to achieve a nuclear explosion it is necessary that the mass of fissile material be more than critical, that is to say, it must be "supercritical." Each act of fission can then result in more than one subsequent fission. The situation depicted in Fig. 2.13, for example, would represent a highly supercritical system, where each fission causes two additional fissions. Thus the first fission is followed by 2 , then by 4 , and by $8,16,32$, etc., in successive generations. Within a very short time, a large number of nuclei are undergoing fission and energy is being released at a very fast rate.

An explosion, nuclear or chemical, results when a large amount of energy is liberated in a very short time within a restricted space. A fission chain in a highly supercritical mass of material can satisfy these conditions. A few pounds of uranium-235 (or plutonium-239) can then be made to generate, within less than a millionth of a second, as much energy as accompanies the explosion of thousands of tons of TNT. This is the basic principle of a nuclear fission explosion.

Because stray neutrons could initiate a chain reaction, a supercritical mass of a fissile isotope might possibly explode. Before the device is actually detonated, each separate mass of fissile material must therefore be maintained in the subcritical state for the existing conditions. Even if stray neutrons started fission chains, such chains would soon die out for lack of neutrons. Consequently, the subcritical mass is completely safe as far as detonation is concerned.

To cause an explosion to occur, the subcritical mass (or masses) of fissile material must be made supercritical within a small fraction of a second. At the same time, free neutrons are introduced to make sure that fission chains are started. 


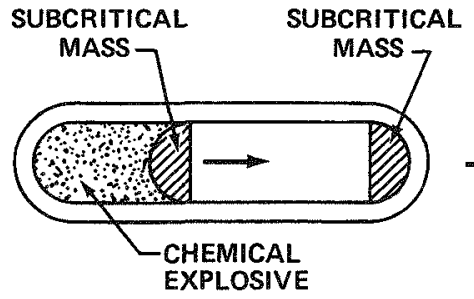

(BEFORE FIRING)

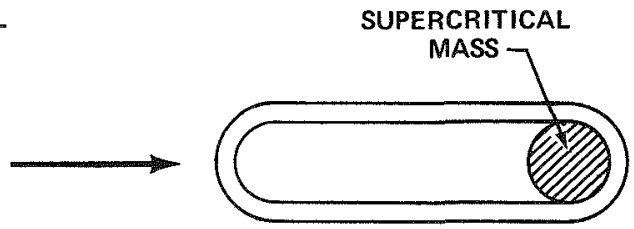

(IMMEDIATELY AFTER FIRING) THEN EXPLODES

Fig. 2.14 Principle of a gun-assembly nuclear device.

There are two ways in which a subcritical mass can be changed into a supercritical one to realize a nuclear explosion. In the first, a subcritical mass of fissile material at one (breech) end of some kind of gun-barrel device is forced rapidly by means of a chemical explosive onto another subcritical mass at the other (muzzle) end, as indicated in Fig. 2.14. The two separate subcritical masses then form a single, highly supercritical mass which explodes when neutrons are introduced. Nuclear devices of this type are known as "gunassembly" systems.

The second method of attaining supercriticality is to subject a subcritical mass of uranium-235 (or plutonium-239) to strong compression. The increase in density resulting from a decrease in volume upon compression can cause the material to become supercritical and explode. The compression may be achieved by surrounding a subcritical sphere of fissile material by an arrangement of chemical explosives designed to produce an inwardly-directed "implosion" wave (Fig. 2.15). Devices depending on compression to attain supercriticality are thus referred to as "implosion" systems.

Provided a nuclear device remains intact it is quite safe. There is a remote possibility that part of the chemical explosive could be detonated accidentally. But the devices are so designed that, if this should occur, the chemical explosion would be accompanied by no more than an extremely small nuclear yield. Furthermore, the danger of sabotage is obviated by an interlocking system of firing mechanisms.

\section{The Fission Products}

It has been mentioned that the lighter nuclei formed when uranium-235 (or plutonium-239) undergoes fission are called fission 


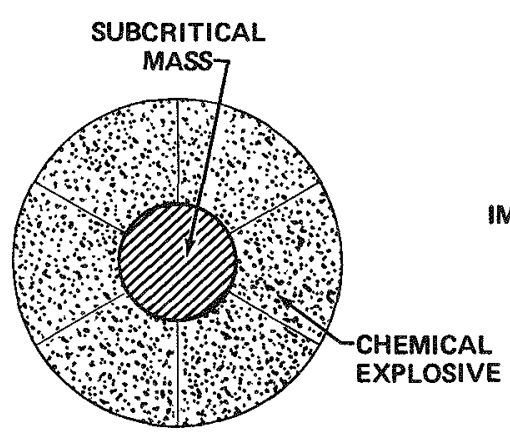

(BEFORE FIRING)

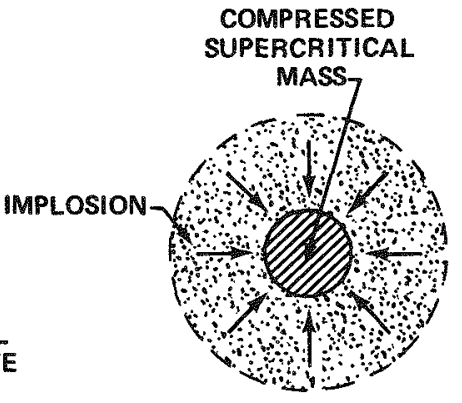

(IMMEDIATELY AFTER FIRING) THEN EXPLODES

Fig. 2.15 Principle of an implosion-type nuclear device.

fragments. Many different fission fragments, also referred to as "initial fission products," are formed in fission. This is because there are forty or so different ways (or modes) in which the fissile nuclei can break apart. Since each act of fission yields two fragments, some eighty different kinds of fission fragments are produced. The different modes of fission do not occur to the same extent; hence the proportions of the individual fission fragments vary over a wide range.

Most, if not all, of the approximately eighty fission fragments are the nuclei of radioactive isotopes of well-known elements with masses ranging roughly from 75 to about 160 . It will be noted that $75+160=235$, which is approximately the mass of the fissile isotopes. The radioactivity is usually manifested by the emission of beta particles, frequently, although not always, accompanied by gamma rays.

As a result of decay, the nucleus of a radioactive isotope is changed into that of another element (p. 17). In the case of the fission fragments, the decay (or daughter) products are generally also radioactive, so that they decay in turn. On the average, there are about three stages of beta-particle emission before a stable (nonradioactive) nucleus is formed (Fig. 2.16). The eighty or so fission fragments thus give rise to around two hundred different isotopes of 36 elements. The general term "fission products" is applied to this complex mixture containing many radioactive isotopes.

The exact composition of the fission product mixture, that is to say, the nature of the isotopes present and their respective proportions, varies with the material (uranium 235 or plutonium- 
239) undergoing fission and with the energy (speed) of the neutrons causing the fission. Regardless of their origin, however, the fission products are a mixture of two hundred or so isotopes, most of which are radioactive.

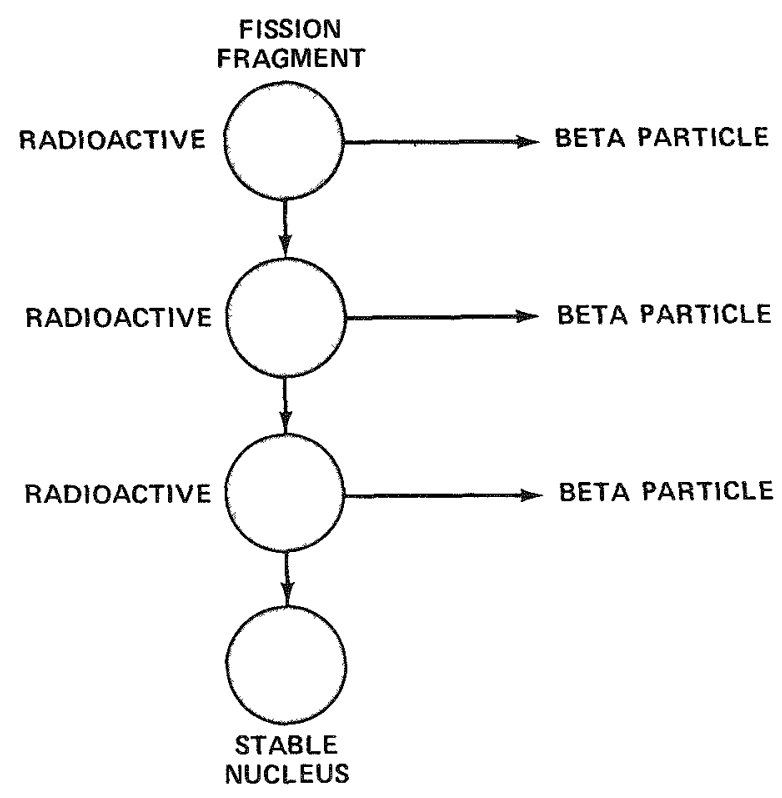

Fig. 2.16 A fission fragment nucleus undergoes, on the average, three stages of beta-particle emission.

Because of the beta particles and gamma rays emitted by the fission products, the latter represent a potential hazard if they should enter the atmosphere or water supplies. This matter will be referred to again later.

\section{Radioactive Decay of the Fission Products}

Every individual radioisotope present among the fission products has its own characteristic half-life; the half-lives of these particular isotopes range from about one millionth part of a second to over a million years. However, the mixture of fission products formed after a nuclear explosion is so complex that it is not possible to express the change in radioactivity of the mixture in terms of a half-life. Nevertheless, it has been found that the decrease in the radioactivity 
with time can be represented (approximately) in a fairly simple manner.

Suppose the radioactivity of the complex mixture of fission products at 1 hour after a nuclear detonation is taken to be 100 units. $^{*}$ Then, at 7 hours after the explosion, the radioactivity will have decreased to about 10 units, i.e., 10 percent of the amount after 1 hour. At $7 \times 7=49$ hours, roughly 2 days, the radioactivity will be down to 1 unit, i.e., 1 percent of the 1-hour value. At $7 \times 7 \times 7=343$ hours, roughly 2 weeks, the radioactivity will be 0.1 unit or 0.1 percent. These results are summarized in the accompanying table.

Approximate Radioactivity of Fission Products

\begin{tabular}{cc}
\hline Time after detonation & Relative activity (percent) \\
\hline 1 hour & 100 \\
7 hours & 10 \\
2 days & 1 \\
2 weeks & 0.1 \\
\hline
\end{tabular}

The rate at which the radioactivity of the fission products decreases in the first few hours after a nuclear detonation is also shown by the curve in Fig. 2.17. It is seen that initially the radioactivity falls quite rapidly as the isotopes of short half-life decay. Subsequently, the decay is at a slower rate. Nevertheless, at 12 hours after the explosion, the radioactivity of the fission product mixture is only about 5 percent of what it was after 1 hour.

It must be emphasized that the change of radioactivity with time described above applies only to the mixture of all the isotopes produced in fission. In some situations, however, individual isotopes, such as strontium-90, iodine-131, and cesium-137, become separated from the mixture. In calculating how the activity decreases in the course of time in these cases the half-life of each particular isotope must be used. Thus, it takes 8 days (the half-life) for the activity of iodine-131 to decrease to half its value at any specified time.

*The basic unit, called a "curie," in which radioactivity is commonly expressed is defined in Chapter 8. 


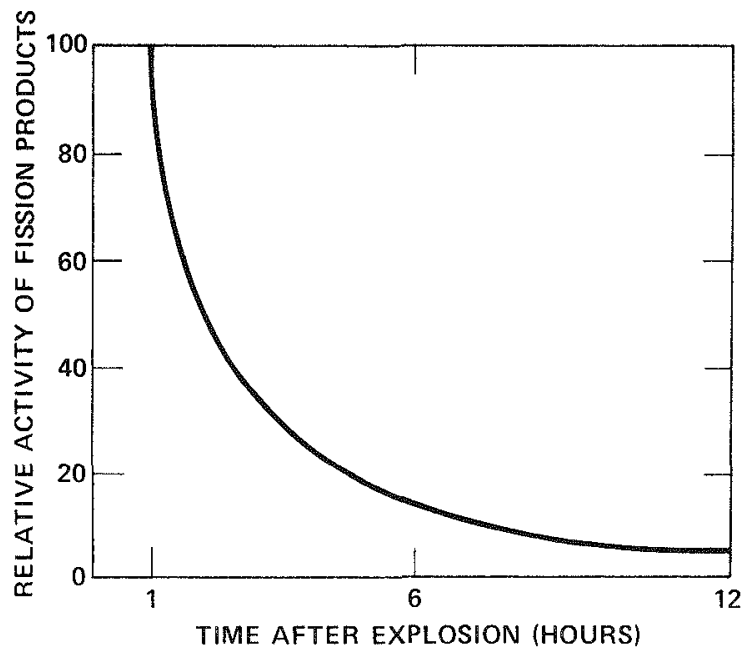

Fig. 2.17 Decay of a fission product mixture. (The total radioactivity is assumed to be 100 units at 1 hour after the detonation.)

\section{Neutron-Induced Activity}

In addition to the fission products, there is another source of radioactivity, especially in an underground nuclear detonation. It has been noted that some of the neutrons liberated in the fission process escape from the fissile substance. These neutrons may be captured by various stable nuclei in the surroundings; the latter include the structural material of the nuclear explosive device itself and the ground in which it is emplaced. As a result of capturing neutrons, many nuclei become radioactive. They then emit beta particles, often accompanied by gamma rays. The radioactivity produced in this way is referred to as "neutron-induced radioactivity" or, in brief, as "induced activity."

The extent of the induced activity depends on the design of the nuclear explosive, since this will determine the proportion of neutrons that escape, and on the particular elements present in the surroundings. The following elements found in rocks are important as far as induced radioactivity is concerned: sodium, silicon, aluminum, iron, and manganese.

There are two other possible sources of neutron-induced activity. If the ground should contain lithium, the interaction of atomic nuclei of this element with neutrons would lead to the formation of 
tritium. This heavy isotope of hydrogen is radioactive. Although tritium is itself a gas, it would generally appear combined with oxygen in the form of water, either vapor or liquid. Ordinary water is represented by the formula $\mathrm{H}_{2} \mathrm{O}$. Usually one hydrogen $(\mathrm{H})$ atom is replaced by one atom of tritium $(\mathrm{T})$, if it is available, thereby leading to the formation of tritiated water (HTO), as depicted in Fig. 2.18.
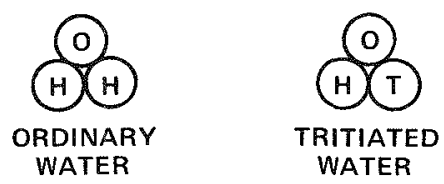

Fig. 2.18 Ordinary water and tritiated water.

When a nuclear explosion occurs in the atmosphere, reaction of the escaping neutrons with nitrogen in the air would give rise to a radioactive isotope of carbon, namely, carbon-14. The carbon would combine with atmospheric oxygen to form gaseous carbon monoxide $(\mathrm{CO})$ or carbon dioxide $\left(\mathrm{CO}_{2}\right)$. However, because there is very little nitrogen in most rocks, no significant quantity of carbon-14 will be formed in an underground nuclear explosion.

\section{Nuclear Fusion Devices}

The most important fusion reactions in nuclear explosive devices involve nuclei of the two heavier isotopes of hydrogen, namely, deuterium and tritium (p.11). The predominant source of energy is the combination (or fusion) of a nucleus of deuterium with one of tritium. In that fusion process a helium-4 nucleus is formed and a neutron is liberated, as represented schematically in Fig. 2.19. At the same time a large amount of energy is released. For equal weights of materials, fusion reactions between the isotopes of hydrogen can liberate three or four times as much energy as in the fission of uranium-235 (or plutonium-239).

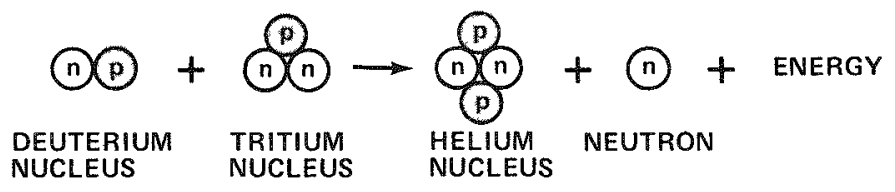

Fig. 2.19 The fusion of deuterium and tritium nuclei. 
In a nuclear explosive device, the fusion reactions take place at very high temperatures-millions of degrees. These high temperatures are generated initially by means of a fission explosion, which serves to trigger the nuclear fusion reactions. Once these reactions between deuterium and tritium nuclei have been started in this way, enough heat energy is produced to sustain the reaction in adjacent material. In contrast to a fission device, where the neutrons maintain the nuclear reaction chain, the chain in a fusion device is maintained by heat. For this and other reasons, explosive nuclear fusion devices are often referred to as "thermonuclear devices." Since the energy is liberated in reactions between hydrogen isotopes, they are also called "hydrogen devices" or, commonly, "hydrogen bombs."

\section{Radioactivity from Fusion Devices}

Fusion reactions as such do not leave any radioactive residues equivalent to the fission products. However, there will be some fission products from the device used to trigger the fusion reactions. Furthermore, a larger number of neutrons will escape from a fusion explosion than from a fission explosion of the same (or similar) energy yield. There will, consequently, be more neutron-induced radioactivity in the former case (Fig. 2.20).

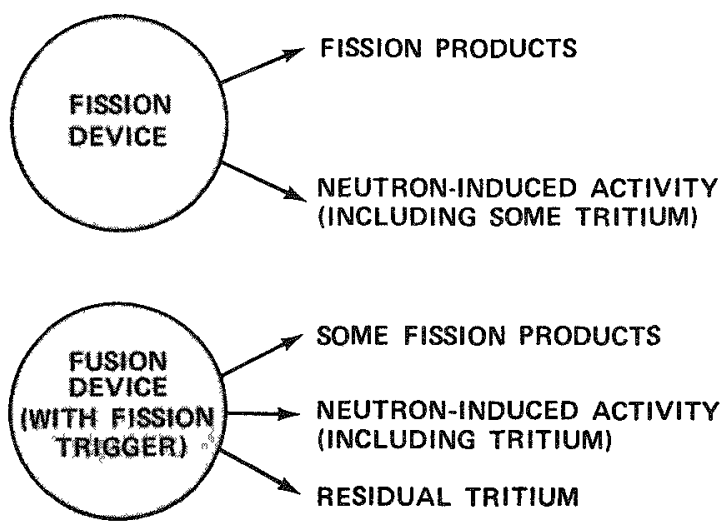

Fig. 2.20 Comparison of residual radioactivities from fission and fusion devices.

An important source of residual activity from the underground detonation of a fusion device is tritium, commonly in the form of tritiated water. The tritium would result in part from interaction of 
the escaping neutrons with lithium in the ground. But even if the rocks contain little or no lithium, some of the tritium from the fusion device itself will remain after the detonation. If lithium is present, then the amount of tritium formed by neutron capture will be greater than from a fission explosion of the same energy yield.

The amount of radioactive material produced in the detonation of a fusion device might be decreased in two ways. First, if the yield of the fission trigger for the fusion reactions is reduced, the quantity of fission products remaining after the detonation is decreased. The proportion of fission to fusion yield is determined by the design of the explosive device and cannot be changed arbitrarily. But exceptionally "clean" explosives have been developed in which the fission yield is only a small fraction of the total energy yield.

The second method for reducing the residual activity of a fusion device is to surround it with a material that absorbs neutrons without becoming radioactive. One such material is the element boron or a compound containing this element. The amount of neutron-induced activity would thus be significantly decreased. It is not always practical, however, to use a boron shield; for example, there may not be sufficient space in the underground chamber where the device is to be detonated. In any event, even if the neutrons were absorbed completely, there would still be some radioactive tritium that had not been consumed in the fusion reactions.

\section{Selection of Nuclear Explosives for Peaceful Projects}

If a nuclear explosive is to be utilized for a peaceful application in the Plowshare program, the explosive can be selected to minimize the potential biological hazard of the associated radioactivity. In an earth-excavation project, for example, the detonation would cause breaching of the surface. As a result, dust particles contaminated with a small proportion of the radioactive products of the nuclear detonation would enter the atmosphere. These particles will descend to earth as "fallout" (see Appendix) and might, in certain circumstances, represent both internal and external hazards.

For an earth-excavation application, therefore, a "clean" explosive device with most of its energy derived from fusion would be preferred. The materials used in the construction of the device would be such as to minimize the formation of those radioisotopes which, because of their chemical and radioactive characteristics, would 
represent a significant biological hazard. In addition, a neutronabsorbing shield would be used, if feasible, to decrease the amount of neutron-induced radioactivity. The quantity of radioactive tritium released from such an explosive would be greater than from a pure fission device of the same energy yield, but most of this would be widely dispersed in the atmosphere in a cratering operation.

Suppose, however, that the nuclear explosion is to be used to stimulate the flow of natural gas by fracturing the rock formation in which the gas is held. The explosive would then be buried at such a great depth that no radioactive material escapes into the atmosphere and there would be no fallout. The natural gas would initially contain gaseous radioisotopes remaining after the explosion, such as tritium and krypton-85 in particular (see Chapter 8 ). In this case, an explosive device would be preferred with most of the energy derived from fission, in order to minimize the amount of tritium. It does not appear possible to eliminate the tritium entirely, because this isotope is produced naturally in a small proportion of nuclear fission reactions.

\section{SUMMARY}

All atoms consist of a central (positively charged) nucleus surrounded by negatively charged electrons. The nucleus carries nearly all the mass of the atom and energy can be produced as a result of rearrangement of the particles (protons and neutrons) contained in atomic nuclei.

Nuclear energy can be released in explosive form either by the fission (splitting) of certain heavy nuclei or by the fusion (joining together) of some very light nuclei. Both fission and fusion explosive devices can be used for peaceful as well as for military purposes.

The residues remaining after a nuclear explosion are radioactive; that is to say, they emit radiations that are a potential hazard to people and animals. The extent and nature of the radioactivity depends on whether the explosive device is based primarily on nuclear fission or primarily on nuclear fusion. When an underground nuclear detonation is intended for a peaceful application, the explosive and its shielding can be selected so as to minimize the potential radiation hazard. 


\section{Chapter 3}

\section{UNDERGROUND NUCLEAR EXPLOSIONS: LOCAL EVENTS}

\section{INTRODUCTION}

In this chapter the local events associated with underground nuclear detonations will be described. This description will provide a basis for understanding the effects that arise from ground motion and from the presence of radioactive debris. The general conclusions are applicable to nuclear explosives of any type, fission or fusion.

The device to be detonated is located in a small underground chamber. The simplest and most commonly used method is to place the nuclear explosive at the bottom of a vertically drilled hole. In some nuclear tests, the detonation chamber is at the end of a tunnel mined horizontally in the side of a hill. The emplacement hole or tunnel is then filled in a suitable manner to prevent the escape of radioactive material into the atmosphere.

The filling operation is known as "stemming." Various stemming techniques have been used in the past and others will undoubtedly be developed in the future. The stemming procedure selected in any particular detonation depends on the circumstances. As a general rule, at the Nevada Test Site, emplacement holes have been stemmed with alternate layers of sand and gravel or of coarse and fine sand (Fig. 3.1). If the nuclear explosive is buried very deeply, as it is in some Plowshare projects, part of the hole could be stemmed with sand and gravel (or similar materials) and the remainder with the mud removed when the emplacement hole is drilled. 
GROUND LEVEL

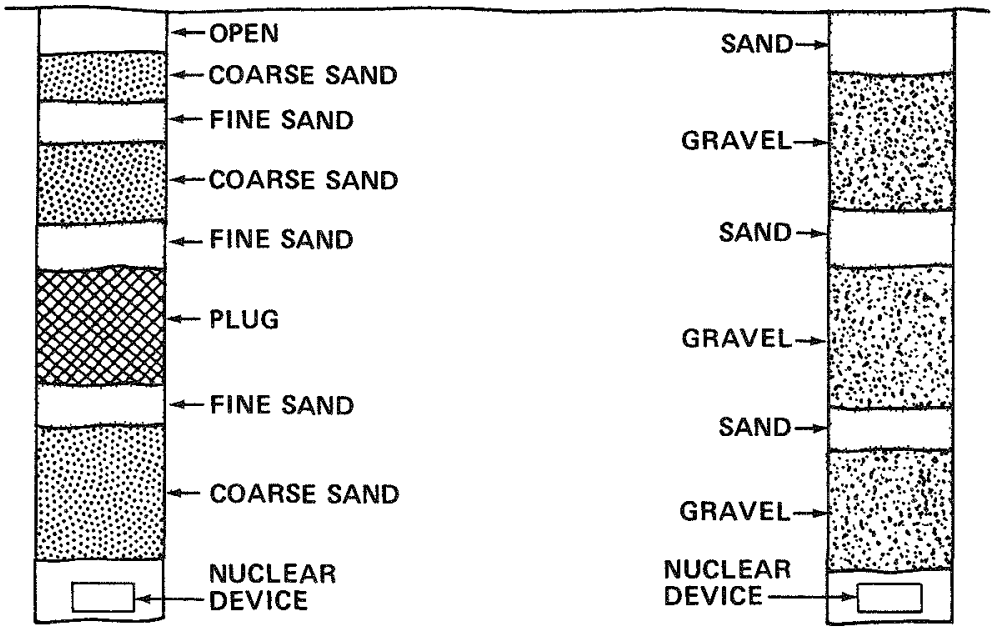

Fig. 3.1 Representations (not to scale) of possible methods of stemming emplacement holes for underground nuclear detonations.

Poured cement or plastic plugs may be used in two ways in an emplacement hole. A plug may be located several hundred feet below the surface to act as a "stemming platform." Its purpose is to support the stemming above the plug and thereby preclude the possibility that all the stemming might fall into the cavity or chimney formed by the explosion (p.39). A plug may also be used to prevent the diffusion of radioactive gases through the stemming material. Such a plug could, in principle, be placed anywhere in the hole, but it is usually located above the stemming platform if one is used.

\section{UNDERGROUND EXPLOSION PHENOMENA}

\section{Formation of Shock Wave}

When a nuclear explosive device is detonated, there is a rapid liberation of large amounts of energy as heat, resulting in temperatures of several million degrees, similar to those in the interior of the sun. This energy, carried predominantly by thermal (heat) radiation, fills the emplacement volume. The radiation is absorbed by a layer of 
surrounding rock which is thus heated and immediately vaporized.* The high-temperature vapor produced in this manner, both from the device materials and from the cavity walls, is at a very high pressure equal to many million times the normal pressure of the atmosphere. (The normal atmospheric pressure at sea level is equivalent to 14.7 pounds per square inch.)

Immediately after its formation, the sphere of vapor, at high temperature and pressure, starts to expand very rapidly. A sharp pressure wave, like a sudden blow, is produced in the surrounding medium. This pressure wave is called a "shock wave," which travels outward through the rock surrounding the detonation point. As it does so, some of the energy of the wave is used in heating the medium and, at the same time, there is an increase in the volume through which the shock energy is distributed. Thus, the pressure at the front of the shock wave (i.e., at the shock front) decreases as it moves farther and farther away.

The shock front forms within a few millionths of a second after the underground detonation. Subsequently, the shock wave propagates through the ground, as just mentioned, and produces certain effects therein. At the same time, changes are occurring in the medium immediately surrounding the detonation point. In order to describe the various effects, it is convenient to examine these two aspects separately.

\section{Propagation of the Shock Wave}

First, the effects of the shock wave as it moves away from the detonation point at high speed will be considered. As it travels through the rock medium, most of the energy of motion of the shock wave is converted back into heat. Initially the temperature is sufficiently high to convert the rock into vapor; then, as the shock front moves farther out, the rock does not get hot enough to vaporize but it is melted. At greater distances from the explosion point, the rock is only crushed and this is followed by a zone in which the medium is cracked extensively.

Farther out, where the shock wave is weaker, the rocks may become permanently distorted. This is described as the "plastic

*The word "rock" is used in this and later chapters in its geological sense as referring to the materials, both consolidated and unconsolidated, that constitute the earth's crust. 
deformation" zone. The weak shock wave, now carrying a few percent of the total energy of the nuclear explosive device, continues to travel outward, and at a considerable distance from the detonation point it will resemble an earthquake wave or "seismic wave." There is then only temporary displacement of the rock; recovery of the original position, after the initial disturbance, is generally accompanied by vibrations in the ground. A wave, like a seismic wave, associated with a temporary distortion is called an "elastic wave."

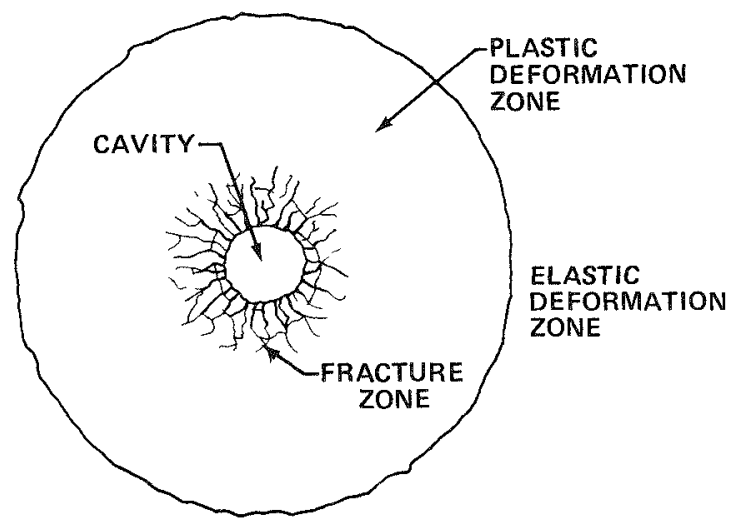

Fig. 3.2 Characteristic zones (not to scale) around an underground nuclear detonation.

The successive zones with increasing distance from the explosion point, as described above, are sketched in Fig. 3.2. The relative dimensions are not meant to be exact; actually, for a given energy yield they will depend on the nature of the rocks that constitute the medium in which the nuclear detonation occurs.

\section{Formation and Growth of Cavity}

Vaporization of material by the shock wave and heat results in the formation of a sphere of vapor, usually referred to as the "vaporization cavity." At this stage, the original material is still contained within its initial volume; hence, there is not strictly a cavity, but rather a sphere of material that has been changed from solid to vapor. The vaporization phase lasts less than a thousandth of a second. 
The size of the vaporization cavity depends primarily on the energy yield of the explosion and on the nature of the rock. For a yield of 1 kiloton TNT equivalent (p.20), the radius of the vaporization cavity in a soft silicate rock is calculated to be about 7 feet. This radius is proportional to the cube root of the yield, as will be explained below. Hence, for an explosion yield of 1 megaton (1000 kilotons) in the same rock, the radius would be roughly 70 feet (Fig. 3.3) at this stage.

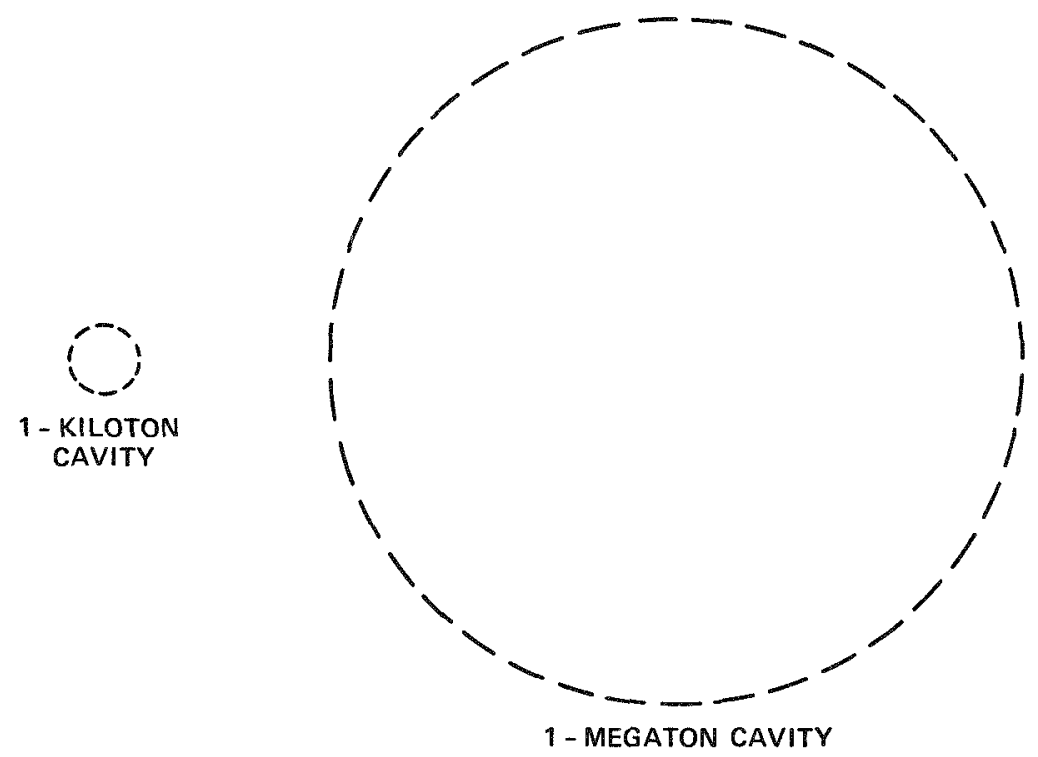

Fig. 3.3 Relative sizes of cavities formed by 1-kiloton and 1000-kiloton (1-megaton) explosions.

The pressure of the vaporized rock in the cavity is now in the neighborhood of a million times the normal atmospheric pressure. This pressure is very much greater than that arising from the weight of the overlying rocks (or overburden). The latter pressure is roughly equivalent to 1 atmosphere for every 15 feet depth of the explosion point; this means that at a depth of 2000 feet, the pressure of the overburden will be less than 150 atmospheres. Consequently, the vapor expands and pushes back the walls of the cavity. Thus, the cavity increases in size until the pressure inside has dropped to the point-a few hundred atmospheres or so-where it is about the same as that due to the overburden. When expansion ceases, the pressure in the "stable cavity" is somewhat greater than the pressure 
due to the overburden because the strength of the material resists the expansion to some extent.

The cube-root relationship between cavity radius and energy yield of the explosion arises from the following considerations. The volume of the cavity in a given rock is expected to be proportional to the energy released. Furthermore, the radius of a spherical cavity is proportional to the cube root of its volume. Hence, the cavity radius should be proportional to the cube root of the energy yield. It turns out that, because of the complex nature of cavity formation, this is not quite true. The approximate cube-root rule is, however, adequate for the present purpose and is applicable to both the vaporization cavity and the stable cavity.

With the formation of a stable cavity, a few tenths of a second after the detonation of the nuclear device, the cavity has reached its maximum size. For a 1-kiloton explosion the radius is now about 50 feet in soft silicate rock and it is proportional to the cube root of the energy yield of the explosion. For the detonation of a 1-megaton TNT equivalent device, for example, the maximum radius of the stable cavity would be roughly 500 feet. The relative sizes of the initial vaporization cavity and of the stable cavity when expansion ceases are shown in Fig. 3.4. For simplicity, the cavity is represented as a sphere, although in practice it will usually be only roughly spherical.

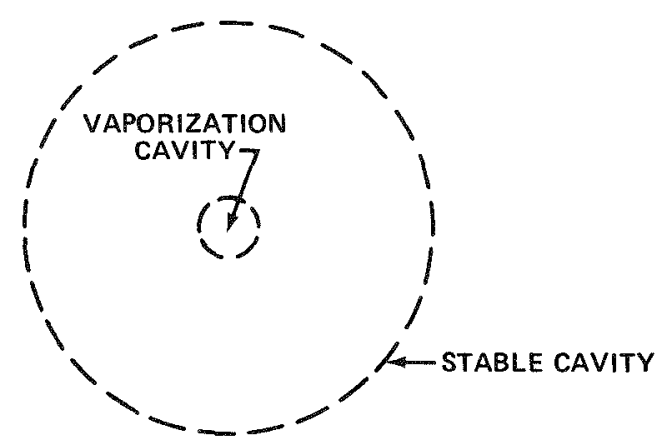

Fig. 3.4 Relative sizes of vaporization and stable cavities.

Since the cavity has been expanding against the pressure of the overburden, its final size will depend to some extent on the depth below the surface. With increasing depth the radius decreases, as will be seen on page 46 . The numbers quoted above are intended to give a 
general indication of what might be expected under average conditions.

When expansion of the cavity has stopped, its walls are lined with many tons of molten rock, some produced by the shock wave and some by the condensation of the rock vapor. Approximately eight times as much rock is melted as is vaporized. The molten rock flows downward and forms a puddle at the bottom of the cavity. Solid rock, crushed by the explosion, may fall from the ceiling and walls and melt in the puddle. Eventually, as it cools, the puddle of molten rock will resolidify to a glass-like mass which traps most of the radioactive residue of the nuclear detonation. Substances that do not condense when the rock resolidifies remain in the cavity initially as gases and vapors.

\section{Collapse of Cavity}

Formation of the rock puddle and its resolidification mark the completion of the phase of cavity growth. The details of the next stage depend to a large extent on the nature of the environment of the detonation point. The vapors and gases in the cavity cool as heat is transferred to the surroundings. As a result, the pressure falls until it is considerably less than that due to the weight of the overburden. There will then be a tendency for the roof of the cavity to collapse. Depending on the natural strength of the rock forming the roof of the cavity, collapse may occur within a few seconds to many days, or it may never occur.

In the Salmon event of October 1964, a nuclear device with a yield of 5 kilotons was detonated at a depth of about 2700 feet in a homogeneous intrusive salt mass or "dome" near Hattiesburg, Mississippi. An approximately spherical cavity, about 57 feet in radius, was formed. More than six years later, however, the roof of the cavity had not collapsed. Evidently, the natural strength of the salt ceiling was sufficient to support the weight of the overburden.

Partial collapse of the ceiling occurred after the Gnome event in December 1961, near Carlsbad, New Mexico. The 3.1-kiloton detonation was at a depth of 1200 feet in a layered salt bed, rather than in a salt dome. Rubble, consisting of large blocks of salt, fell from the roof until the cavity was roughly half filled. Collapse then ceased. The cavity now remaining is very roughly hemispherical, 134 to 196 feet wide and some 75 feet high. A photograph taken inside 


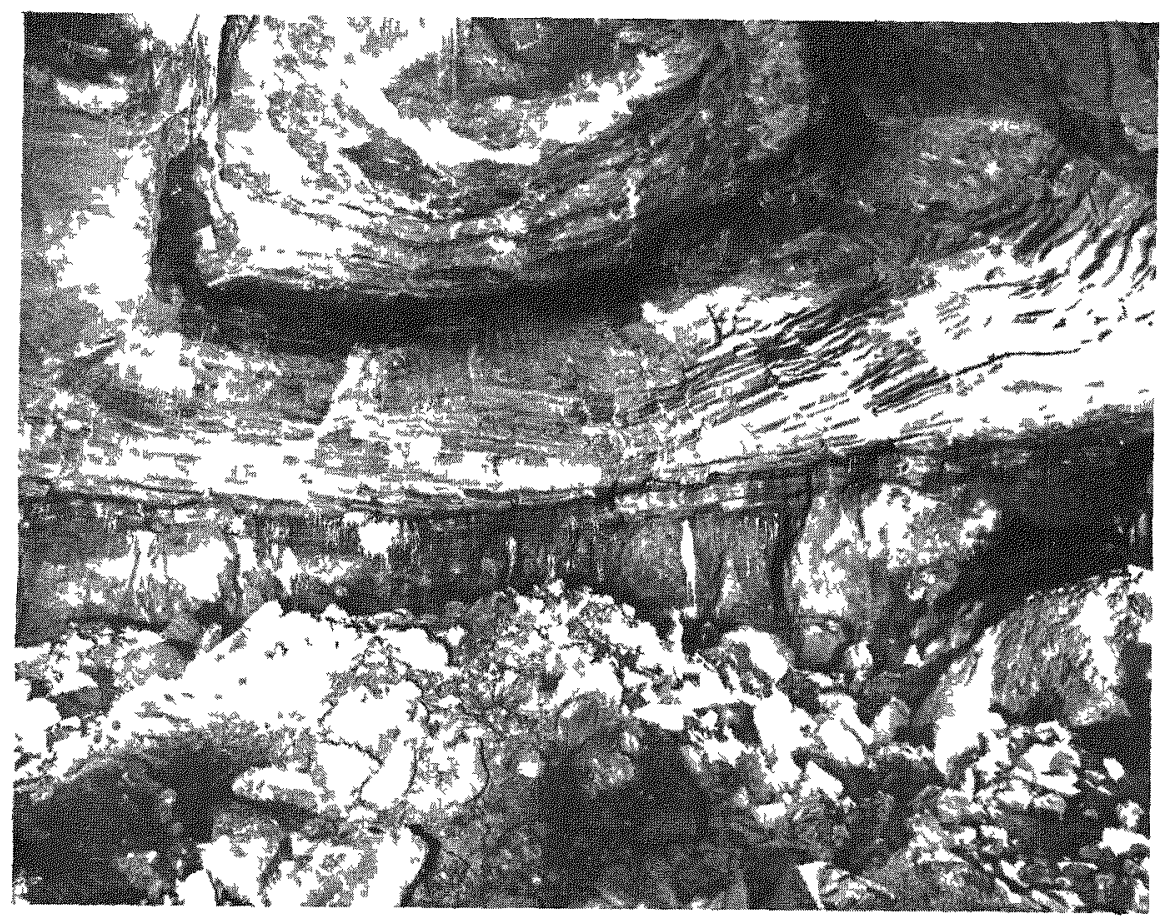

Fig. 3.5 The Gnome cavity.

the Gnome cavity is reproduced in Fig. 3.5. The size may be compared with that of a man seen standing on the fallen rubble just right of center.

\section{Formation of Chimney}

In most underground detonations, the roof is not strong enough to support the overburden. Consequently, the entire cavity ceiling falls into the cavity and this 1s followed by the progressive collapse of overlying rock. Within a short time, a tall cylinder, commonly referred to as a "chimney," is formed in the rock. The volume of the chimney is much greater than that of the original cavity and it is filled almost to the top with broken rock (or rubble). As a general rule, a small empty space remains at the top of the chimney (Fig. 3.6).

If the collapse of the chimney material reaches the surface, a depression called a "subsidence crater" will be formed (Fig. 3.7). An 


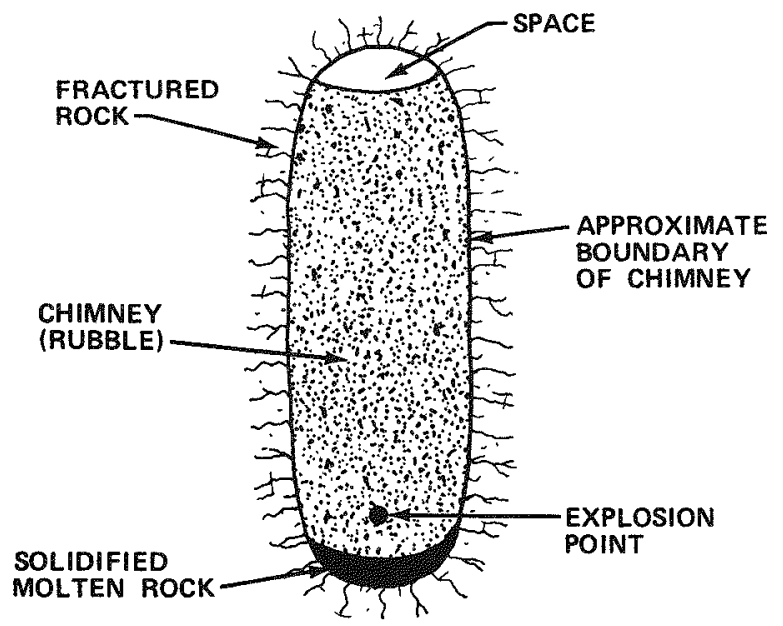

Fig. 3.6 The rubble chimney formed after collapse of the cavity in an underground nuclear detonation.

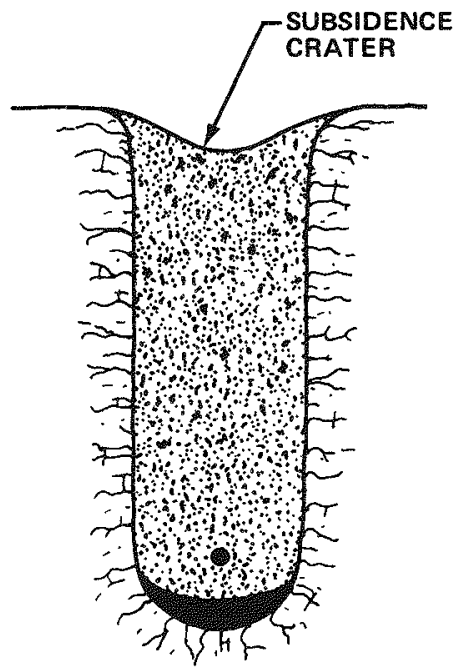

Fig. 3.7 Formation of a subsidence crater in an underground nuclear detonation.

aerial photograph of such a crater taken at the instant of formation is given in Fig. 3.8. On the other hand, if the top of the chimney is well below the surface, a low dome may remain. Here the earth has been pushed upward by the shock wave and stays in the raised position even after the chimney has formed. 


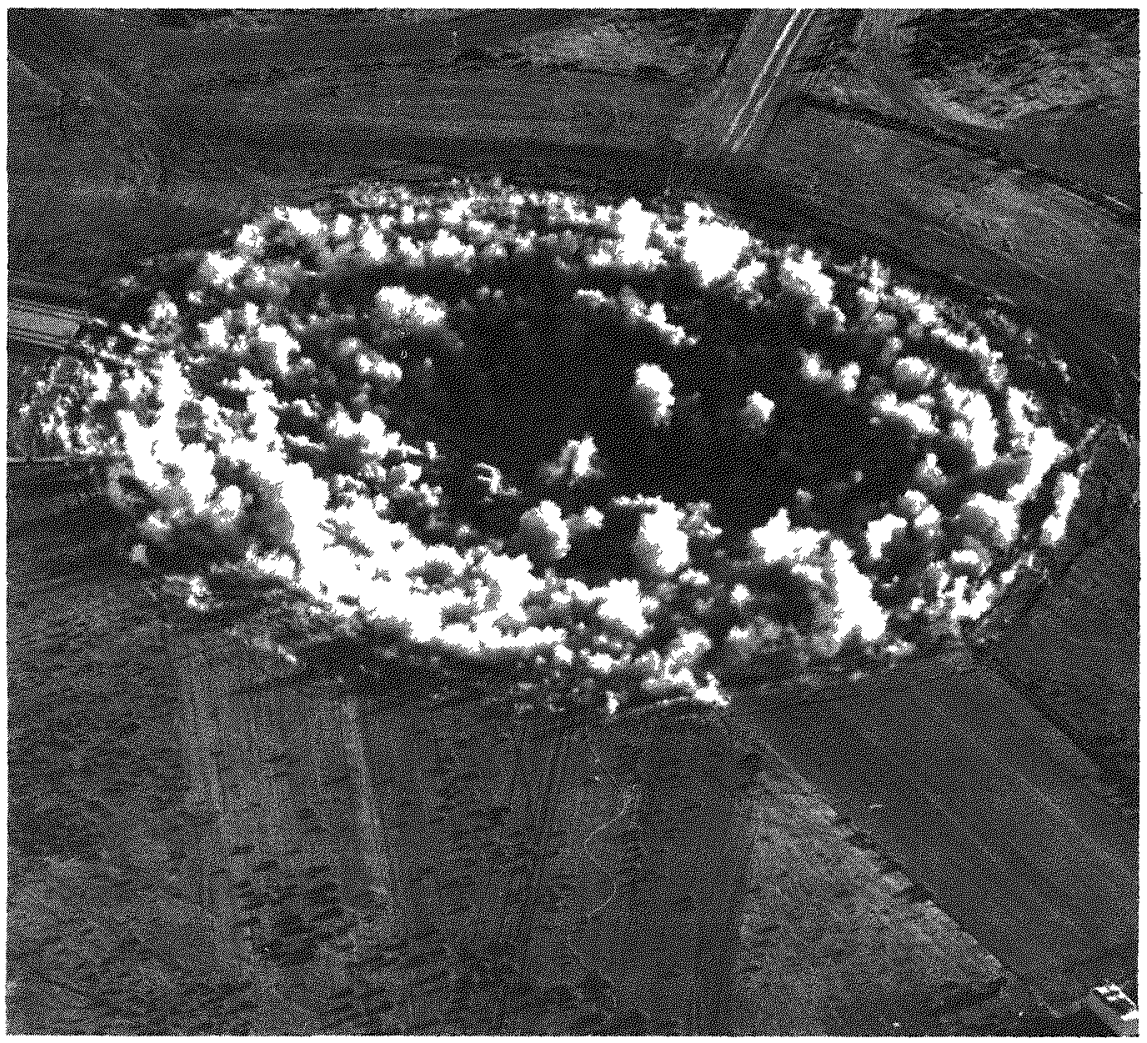

Fig. 3.8 Subsidence crater at the instant of formation, accompanied by dust clouds.

\section{Reflection of Shock Wave at the Surface}

The foregoing discussion has referred to nuclear detonations at such depths that there is no breaching of the surface. Explosions of this type, which represent the great majority, are said to be "contained." To see what distinguishes a contained explosion from one that is not contained, the behavior of the shock wave in the ground must be examined further. This wave travels in all directions away from the detonation point, but for the matter of immediate interest, only the motion upward, toward the earth's surface, needs to be considered.

When the shock wave reaches the surface, it is reflected back into the ground (Fig. 3.9). The reflected wave, however, is not a pressure 


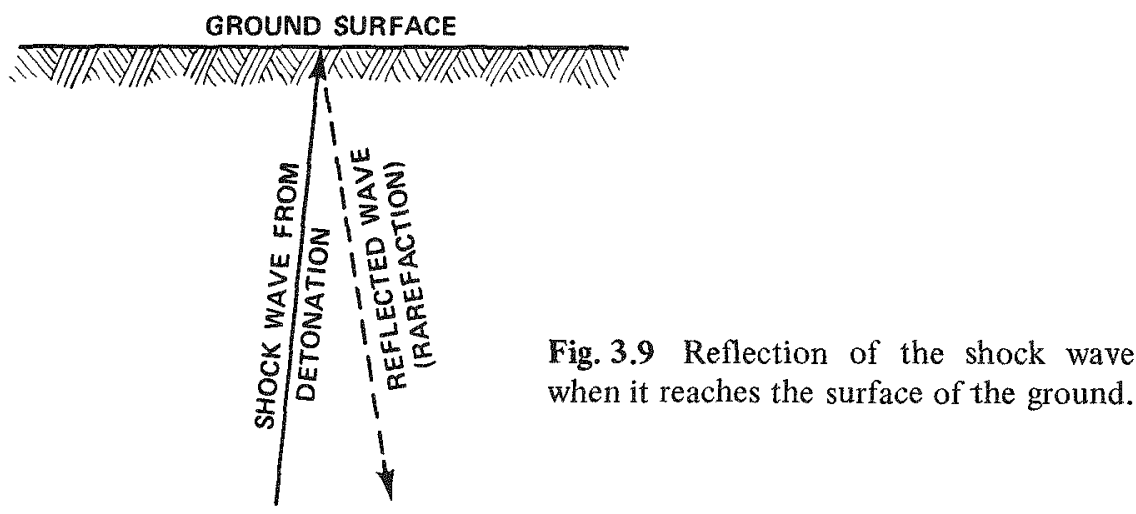

wave; it is, in fact, just the reverse. It is a tension wave, generally referred to as a "rarefaction wave," in which the pressure is less than in the surrounding medium. In the outgoing shock wave, on the other hand, the pressure is greater than in the surroundings.

In a contained underground explosion, the depth of burial is such that, by the time the reflected wave reaches the cavity formed by the explosion, the cavity has ceased to grow. Furthermore, the reflected wave, when it does reach the cavity, is so weak that it has no effect on cavity growth. In other words, in a contained explosion, because of the substantial distance between the detonation point and the surface, the cavity will have become stabilized before the return of the weak reflected wave. In an explosion at a smaller depth, however, the moderately strong reflected wave could reach the cavity while it is still growing, thereby assisting its upward growth. A breakthrough to the surface might then occur (see Appendix).

It may be noted, in passing, that when the shock wave is reflected back into the ground, there may be an upward separation of one or more layers of rock and earth near the surface. This phenomenon is called "spalling." It is significant for contained explosions that are not too deeply buried, but is observed with practically all underground nuclear detonations.

\section{CALCULATION OF EFFECTS OF UNDERGROUND DETONATIONS}

\section{Basis of the Calculations}

An important aspect of the safety program associated with underground nuclear detonations is the capability for calculating the 
expected behavior of the medium around the explosion point. The main purpose of these calculations is to predict the effects of the detonation before it is conducted. There are some uncertainties in the model used in the calculations, but by comparison of the predicted effects with results obtained in actual detonations the model can be refined. As a result, the predictions become more reliable.

The calculations are made by the use of appropriate computer codes which have been specially developed for this particular purpose. The treatment is based on well-known fundamental laws of physics, but it requires a knowledge of the properties of the rocks through which the shock wave travels. These properties are partially known from field and laboratory experiments, but sometimes assumptions must be made; in addition, use is made of the results of measurements in previous tests.

The initial condition for the calculations is that the energy corresponding to the expected (or actual) yield of the explosion is deposited in a given space, such as a sphere. The volume of this sphere is taken to be equal to that of the rock chamber where the nuclear device is detonated. The extent to which the shock wave causes vaporization of the surrounding medium can then be computed provided the "heat of vaporization" of the rock is known. This quantity, a measure of the heat required to convert the rock into vapor, can be derived from laboratory experiments.

The next step is to determine the volume of the rock vapor at the temperature and pressure in the cavity. For this it is necessary to know the relationship between the temperature, pressure, and density of the vapor over a wide range of conditions. Such a relationship is commonly referred to as the "equation of state" of the rock vapor. Because there are no experimental measurements at the existing very high temperatures and pressures, the equation of state is derived from assumptions based on accepted theories.

\section{Calculation of the Cavity Radius}

The volume, and hence the radius, of the stable cavity when rock vaporization ceases can now be calculated. Subsequently, the resulting high-pressure gas expands against the outside pressure due to the overburden. By using the assumed equation of state, it is possible to determine the radius of the stable cavity formed in a given material at a specified depth of burst. 
From calculations of the rate of motion of the rock surface, the manner in which the cavity radius increases with time can be evaluated. Typical results for a deep underground detonation are shown by the curve in Fig. 3.10. The radius is seen to increase, at first rapidly and then more slowly, until the stable value is attained. Eventually, of course, the roof may collapse and fill the cavity with rubble.

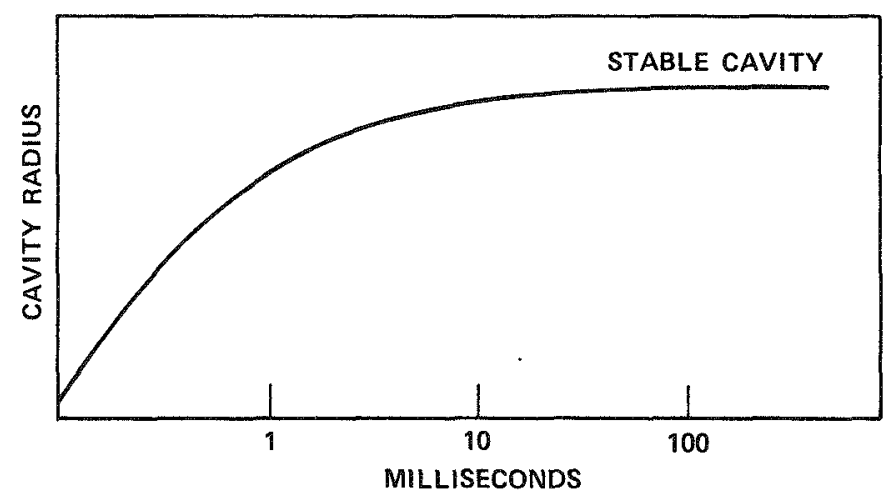

Fig. 3.10 Calculation of the growth of the cavity in an underground detonation.

The size of the stable cavity depends on the energy yield of the nuclear device, on the nature of the rock medium in which the detonation occurs, and to some extent on the depth of the explosion point. As far as the yield is concerned, the situation is fairly simple in principle. Theory requires that the radius in a given rock should depend on the cube root of the energy yield, as mentioned on page 38 . Therefore, to obtain the radius for any specified yield, all that should be necessary is to multiply the cavity radius for a 1 -kiloton explosion in the same rock by the cube root of the specified energy yield. As stated earlier, this rule is approximate and a better relationship, based on actual measurements, is commonly used.

Allowance for the nature of the rock medium in the immediate vicinity of the detonation is made by using the appropriate heat of vaporization and equation of state. These properties may vary significantly from one rock type to another, and calculations of the cavity radius have been made for several media. Some values of the radius of the stable cavity at the same depth in different rock types 
obtained in this manner will be given shortly (see Fig. 3.11). An alternative possibility is to use factors derived from previous measurements of the actual radius of the stable cavity in various media.

The deeper the explosion point, the greater will be the weight of the overburden that opposes expansion of the vapor cavity. The size of the cavity would thus be expected to be smaller as the nuclear device is detonated at greater depths. A relationship between the radius of the stable cavity and the depth has been derived from theoretical considerations and this seems to be quite satisfactory. The radius in a given medium is found to be inversely proportional to the one-fourth power of the depth of the explosion.

\section{Stresses in the Rock}

The shock wave is now followed as it travels through the medium surrounding the cavity. The high pressure at the shock front produces what is called "stress" in the material. This may be defined for the present purpose as the force (per unit area) generated in the rock in resisting the effect of an external force, namely, that due to the shock pressure. Stress can cause an internal displacement or distortion, referred to in this respect as "strain." In turn, this displacement of material can cause stress in adjacent material and so on throughout the rock medium.

The fundamental equations of mechanics provide a connection between the applied stress and the resulting acceleration of each point in the medium around the detonation zone. By a process of integration, which is a form of addition, over a short period of time, the acceleration gives the velocity of the material at each point. Integration of the velocity, in turn, gives the displacement of the point during the specified short interval of time. From the displacement, a new value of the stress is determined and the calculations are repeated by a computer, over and over again, for a series of successive time intervals. During these intervals the shock wave is moving farther and farther from the explosion point. Thus, the stresses can be evaluated for considerable distances in the surrounding rock.

The foregoing calculations require a knowledge of the equation of state of the medium within which the shock wave has a noticeable effect. If this medium is a single rock type, such as granite or shale, then only one equation of state is required. On the other hand, if the 
shock wave, as it moves outward, encounters other rock formations, the computations must take into account the change in the equation of state at the appropriate distance. Hence, the geology of the region must be understood if reasonably satisfactory predictions are to be made of the effects of an underground nuclear detonation.

Rocks have various "strength" properties which can be determined from laboratory tests. The applied stress must exceed a certain strength value before the rock can be crushed or cracked. If the stress is less than this strength, then there may be only plastic (permanent) or elastic (temporary) deformation. Consequently, by comparing the calculated stresses at various distances out from the explosion point with the known strength of the rock material, the range of cracking can be determined.

The results of calculations made for four different rock types, namely, dolomite, granite, sandstone, and shale, are represented in Fig. 3.11. The values of the stable cavity radius and of the limit of
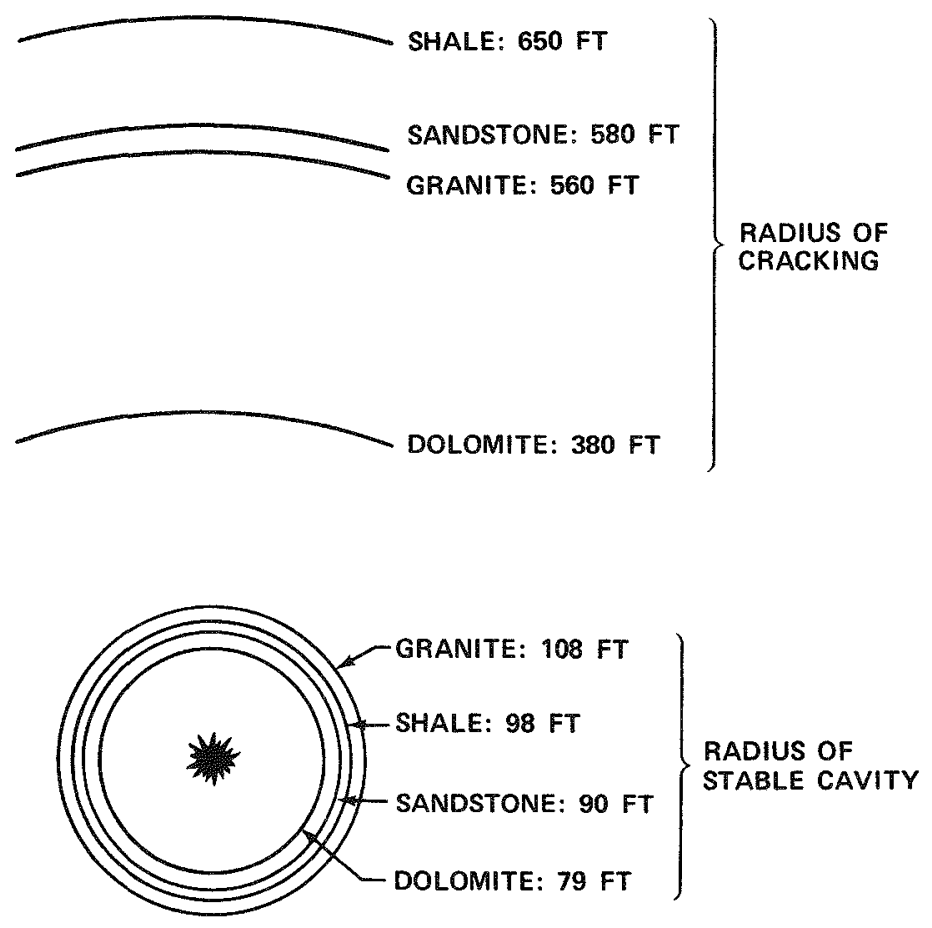

Fig. 3.11 Stable cavity and cracking radius calculated for a 30-kiloton explosion at a depth of about 1000 feet. 
cracking are for a detonation of 30 kilotons TNT equivalent at a depth of about 1000 feet. The radius of the cavity does not necessarily vary with the nature of the rock medium in the same way as does the cracking distance, because they depend on different properties of the rock.

\section{Dimensions of the Chimney}

It has been seen that if the explosion is contained, the roof of the cavity usually collapses and a chimney is formed. Although it is not yet possible to calculate the radius and height of the chimney from first principles, some indication of the height can often be obtained from other considerations, such as the radius of the fracture zone.

For example, for a 60-kiloton detonation at a depth of about 1540 feet in granite, calculations indicated that the rock material would be cracked to a distance of at least 800 feet above the explosion point. It was expected, therefore, that if the roof of the cavity collapsed, as it did, the chimney height would be 800 feet or more. The actual height was a little over 920 feet.

A different situation arose in connection with the Gasbuggy gas-stimulation test near Farmington in northwestern New Mexico (December 1967). The 29-kiloton nuclear device, at a depth of 4240 feet, was in a layer of shale. Above this was a layer of sandstone, overlain by coal ( 3915 to 3882 feet), and other layers which are not important for the moment. It was expected that the coal, which is easily compressed, would absorb the shock energy. Cracking and collapse should thus not extend much above the base of the coal bed. This actually proved to be the case. The top of the chimney was close to the bottom of the coal layer.

If other procedures are not available, a rough estimate of the chimney dimensions, for a detonation in a single rock type, may be made in the following manner. The chimney is assumed to be a cylinder with a radius equal to that of the stable cavity before collapse. To obtain the approximate height of the chimney, the radius is multiplied by a factor that depends largely on the nature of the rock in which the chimney is formed. The value of this factor, which is commonly in the range of 3 to 6 , is derived from actual measurements in that medium made at previous detonations.

\section{Comparison of Calculations and Observations}

Agreement between the calculated results and actual observations is generally quite good and is getting better. As more accurate 
equation-of-state data are obtained for a variety of rock types and more is understood about the strength characteristics of these materials, the predictions can be improved.

As an example, reference may be made to the Salmon test in a salt dome (p.39). The first computations indicated a radius of about 80 feet for the stable cavity. Subsequently, when better information concerning the strength of the salt became available, a recalculation gave a value of approximately 54 feet for the radius.

It will be recalled that the roof of the cavity produced in the Salmon event did not collapse. Consequently, it was possible to determine the cavity volume by pumping air into the cavity and measuring the accompanying increase in pressure. By assuming a spherical cavity, the radius was found to be roughly 57 feet. This is quite close to the value ( 54 feet) derived from the calculations using the better strength data.

There are more detailed ways of checking the accuracy of the calculations of the effects of underground detonations. Such properties as the variation of the maximum (or peak) stress with distance from the explosion point can be measured as well as calculated. Another quantity which can be measured is the maximum (or peak) velocity of the surrounding material at various distances from the detonation. Furthermore, the time at which the shock front in the rock surrounding the explosion point arrives at different locations has been observed and has also been calculated.

At many underground nuclear detonations, instruments are placed at various distances from the explosion to measure the peak stress, the peak particle (material) velocity, the time of arrival of the shock front, and other quantities of interest. The instruments near the nuclear device are destroyed, but before their destruction the readings are transmitted by cable and recorded at a safe distance.

The measurements are subsequently compared with the values predicted by the computer codes. If there are any significant differences, an effort is made to determine their cause, so that subsequent calculations can be improved. In Fig. 3.12, for example, the circles and dots give the observed peak (radial) stress and peak velocity of the material, respectively, at various distances from the explosion point in the Salmon event. The curves, on the other hand, represent calculated results.

It is seen that the measurements give somewhat higher values than do the calculations. This discrepancy can be removed by using a 


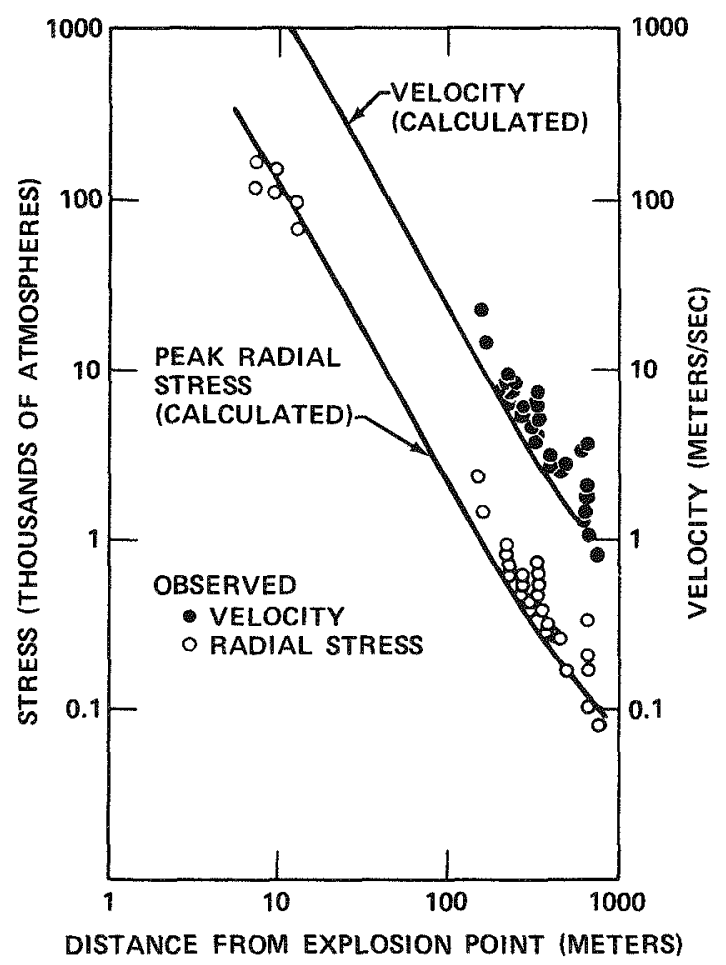

Fig. 3.12 Comparison of observed and calculated stress and particle (material) velocity for the Salmon test.

better equation of state for the salt. The computed results are then found to agree well with those recorded by the various instruments.

Pressures and Temperatures in the Cavity

The calculations described above are mainly concerned with the shock wave and its effects. An entirely different type of calculation is used to determine how the temperature and pressure of the cavity gas change with time as cooling proceeds. The cooling is considered in two separate stages: first, from the time the cavity ceases to expand until cracked material begins to drop from the roof and collapse starts, and second, during the period of collapse when relatively cool material is falling into the cavity.

From the limited measurements available, the agreement between the calculated pressures in the cavity and the observed values seems 
to be good. But more experimental data are needed to check the accuracy of the computations. Consequently, attempts will be made to observe the change in pressure, and possibly also of the temperature, of the cavity gas in future underground nuclear detonations. With the aid of such measurements, the calculations can be put on a firmer basis.

\section{General Conclusions}

The general conclusions to be drawn are that suitable methods are being developed for calculating the effects in the immediate vicinity of an underground nuclear detonation. If the geology of the site is known in detail and if equations of state and strength properties of the materials are available, the calculations may predict quite accurately what might be expected from a detonation of a specified yield at a given depth.

\section{CONFINEMENT OF RADIOACTIVITY IN UNDERGROUND EXPLOSIONS}

\section{Condensation of Vapors}

When the underground explosion cavity has reached its maximum size and become stabilized, just before the roof collapses, the temperature has dropped sufficiently for most of the vapors to condense. The chief gases and vapors still remaining are water vapor (from moisture in the rocks), possibly carbon dioxide (from carbonate rocks such as dolomite), krypton, xenon, and some iodine (from fission products), and tritium or tritiated water (from a fusion device or from the interaction of neutrons escaping from the nuclear detonation with lithium in the rock material).

When collapse occurs and rubble falls into the cavity from the roof, the gases and vapors remaining are cooled. As a result, most of the water vapor and iodine will condense, but the carbon dioxide, krypton, xenon, some tritium, and part of the iodine will remain as "noncondensable" gases. Only a fraction of the tritium will be in the gas; since tritium is an isotope of hydrogen, some of the hydrogen atoms in water will be replaced by tritium atoms (p. 29). Hence, part 
of the tritium will be left behind with the condensed water as tritiated water.

\section{Possible Escape of Radioactive Material}

There are two types of possible accidental release of radioactive material to the atmosphere from an underground explosion. One, called "seepage," is the slow diffusion of the noncondensable gases through the rubble in the chimney to the earth's surface. The other, which occurs quite quickly, is the release of both gases and particulate matter. It is referred to as "prompt venting" or merely as "venting."

Seepage of gases to the ground surface is hindered by the rubble in the chimney. In addition, the condensation of the vapors in the chimney causes the pressure to drop, possibly below the existing atmospheric pressure. Thus, unless significant amounts of carbon dioxide are present, the pressure driving the residual gas and vapor through the interstices in the rubble toward the surface is usually quite small. In fact, there may be a tendency for gases to move into the chimney rather than out of it.

Should seepage occur, it may commence from as early as a few minutes to as late as several hours after the detonation. As a general rule, it continues for a few hours but in unusual instances seepage has persisted for a few days. The amount of radioactivity released in this manner (as well as in prompt venting) has, however, never been more than a very small fraction of that formed in the nuclear detonation.

Prompt venting can apparently arise from the availability of a path of relatively low resistance that permits escape to the surface of radioactive debris, both gases and particles, under the pressure generated by the explosion. The few ventings that have been experienced have usually resulted from what might be described as mechanical problems, such as failures in stemming or closure devices, especially in tunnels, or in "line-of-sight" pipes leading to the chamber containing the nuclear device.

\section{Occurrence of Accidental Releases}

From the signing of the limited test ban treaty (p. 1) in August 1963 to the end of 1970, the United States has conducted 225 
announced underground nuclear detonations with yields ranging from less than a kiloton to more than one megaton (1000 kilotons) of TNT equivalent. These detonations were performed either in deep holes or in hillside tunnels at depths sufficient to prevent breakthrough to the surface. Furthermore, the stemming was considered to be adequate, so that no seepage or venting was expected. In seventeen instances, all at the Nevada Test Site, measurable radioactivity was observed off the Site.

In some of the detonations in which radioactive material escaped, the gases vented through ground fissures, starting within less than a minute after the explosion. In other instances, seepage of radioactivity was not detected until surface subsidence occurred. In no case did the radiation levels and doses at populated off-site areas approach the radiation protection guides recommended by the Federal Radiation Council for normal peacetime activities (see Chapter 8). (Details of the extent of these releases may be found in the monthly "Radiological Data and Reports," issued formerly by the U.S. Department of Health, Education and Welfare and now by the Environmental Protection Agency.)

An examination of the seventeen tests in which seepage or venting occurred shows that thirteen were either tunnel experiments and/or experiments involving line-of-sight pipes part way through the stemming. Thus, in only four fully buried and completely stemmed detonations did significant leakage of radioactivity occur.

Another point of interest is that in fifteen of the seventeen cases, the energy yields of the explosions were in the low range, that is, less than 20 kilotons. In the other two events accompanied by escape of radioactivity, the yields were in the low-intermediate range (20 to 200 kilotons). No off-site release of radioactivity has been observed in any underground detonation with a yield exceeding 50 kilotons.

The significance of these results, and the possible reason, will be considered shortly. But first, something must be mentioned about one of the tests of low-intermediate yield in which radioactivity escaped into the atmosphere. In this particular test (Nash, January 1967), the cavity extended into dolomite (carbonate) rock and large quantities of noncondensable carbon dioxide gas were apparently liberated by the heating of the dolomite. It has been suggested that, because of the porosity of the medium, some of the carbon dioxide diffused to the ground surface carrying with it radioisotopes of other noncondensable gases. 


\section{Scaled Depth of Burial}

In underground detonations that are designed to be contained, the larger the expected yield of the nuclear explosion the greater the depth at which the device is buried. In order to compare depths of burial for explosives of different yields, it is customary to define a "scaled" depth of burial. Based on considerations similar to those discussed on page 38 , this is obtained by dividing the actual depth of burial in feet by the cube root of the energy yield in kilotons. Suppose, for example, that the yield of the device to be detonated is 27 kilotons, and the actual depth of burial is 1200 feet. The cube root of 27 is 3 and so the scaled depth of burial is then $1200 / 3=400$ feet. $*$

It was thought at one time that if the scaled depth of burial was the same for nuclear devices of different energy yields, the behavior with regard to the containment of radioactivity would be the same. But this does not appear to be true. For a given scaled depth of burial, seepage of radioactivity has occurred on a few occasions with nuclear explosions of low yield, but rarely with those of higher yield.

The seepage problem has been studied by drilling holes down from the surface into the chimney some time after the detonation and determining the level at which radioactivity is first detected. The results confirm the conclusions reached from observations of actual seepage. For detonations with energy yields up to about 10 kilotons, the radioactivity is encountered near the surface. But for higher yields, the distance from the surface at which radioactivity is detected increases with increasing yield, for the same scaled depth of burial.

Clearly, the actual depth of burial is important and must be considered as well as the scaled value. For a scaled depth of burial of 400 feet, the actual depth for a 1-kiloton detonation would be 400 feet, whereas for a $27-$ kiloton explosion it would be 1200 feet (Fig. 3.13). Thus, for the 1-kiloton device, the noncondensable gases have to travel only 400 feet to reach the surface, compared with 1200 feet for the 27-kiloton device.

It appears, therefore, because of the shorter distance from the explosion point to the surface, that the gases are more likely to escape from a detonation of lower yield than from one of higher

*The correct units for the scaled depth of burial are feet/(kilotons TNT) ${ }^{1 / 3}$. For simplicity, however, the scaled depth is stated in feet in this book. 
yield at the same scaled depth of burial. It is true that more gas is liberated in the latter case, but its pressure, which provides the driving force for the seepage, would be about the same regardless of the yield of the explosion. Although the quantity of gas increases in

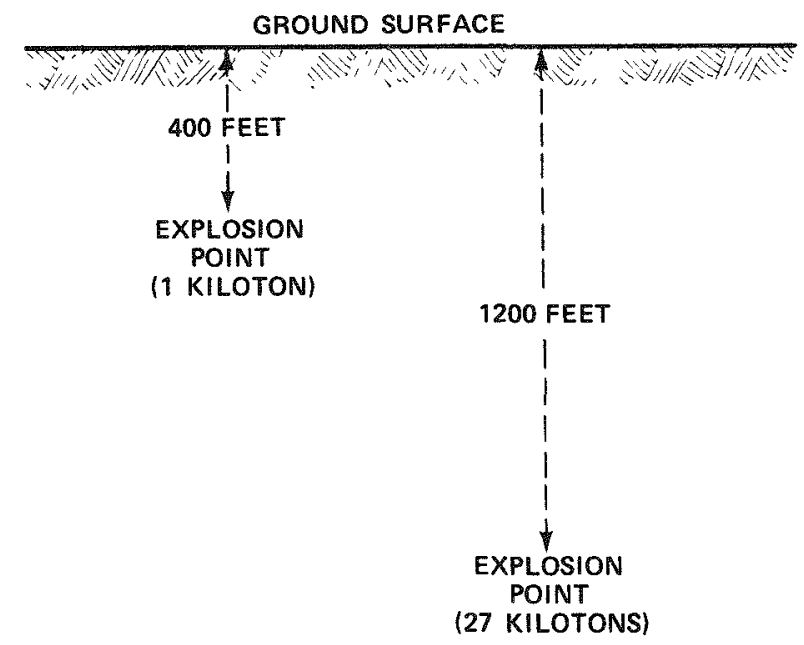

Fig. 3.13 Comparison of actual depths of burial for the same scaled depth.

proportion to the yield, so also does the volume occupied by the gas; hence, the pressure is (approximately) independent of the yield.

\section{Prevention of Seepage of Radioactivity}

The observations described above show that, if the escape of radioactive gases by seepage is to be avoided, the scaled depth of burial should be greater for nuclear devices of lower energy yield than for those of higher yield. However, since radioactivity escaping from an explosion of high fission yield might be serious if it should occur, scaled depths of burial are based on those required for low yields. Thus, there is an additional safety factor included in selecting the depth of burial for an underground nuclear detonation of high fission yield.

The minimum scaled depth of burial currently used for explosions that are designed for complete containment in tuff or dry alluvium is normally greater than 350 feet, even for nuclear explosive 
devices of high yield.* Furthermore, the actual depth of the explosion point is seldom less than 500 feet.

\section{Venting Through Cracks and Faults}

Many rock formations normally have gross structural discontinuities such as joints and faults. These geologic features will be considered more fully in Chapter 4 , but for the present it is sufficient to say that they are associated with breaks in the rocks. There is a possibility that, in an underground nuclear detonation, gases and solid particles may be vented through such natural breaks, as well as through cracks (or fractures) produced by the explosion.

A number of faults have been identified at the Nevada Test Site. Several nuclear devices have been detonated near these faults and in certain instances displacement of the fault has been observed (see Chapter 6). In a few cases, venting of radioactive material has occurred through cracks in the ground. The devices were all in the low yield (less than 20 kilotons) range. Fault motion and fracturing have been noted in explosions of much higher yield, but there was no venting, presumably because of the greater depth of burial.

There are reasons, based on experience, for believing that natural (or other pre-existing) cracks in the immediate vicinity of the expanding cavity in an underground detonation are not significant vent paths. It appears that such cracks that may have extended into the cavity or were a short distance from it are closed by the pressure of the shock wave. Consequently, the cracks no longer exist as such but rather they become tightly sealed fractures.

In some situations, pre-existing cracks may begin to open up again after the shock wave has passed. The same thing could happen to rock fractures near the cavity that are produced by the explosion. These cracks may sometimes progress backward toward the cavity. Molten rock, which now lines the cavity wall, will then be forced into the cracks by the pressure of the gases and vapor in the cavity. Because of the lower temperature the molten rock resolidifies

*There are several varieties of tuff, but they are all composed of compacted volcanic ash. Alluvium consists of particles of rock, sand, clay, etc., that have been deposited from water over long periods of time and have become partly compacted. Much of the earth's surface is covered with alluvium. 
immediately and seals the cracks. There is thus a tendency for possible vent paths close to the cavity to be shut off.

The sealing of cracks in this manner has been observed on several occasions when excavations have been made after nuclear explosions. An example of the phenomenon is given in Fig. 3.14; the photograph

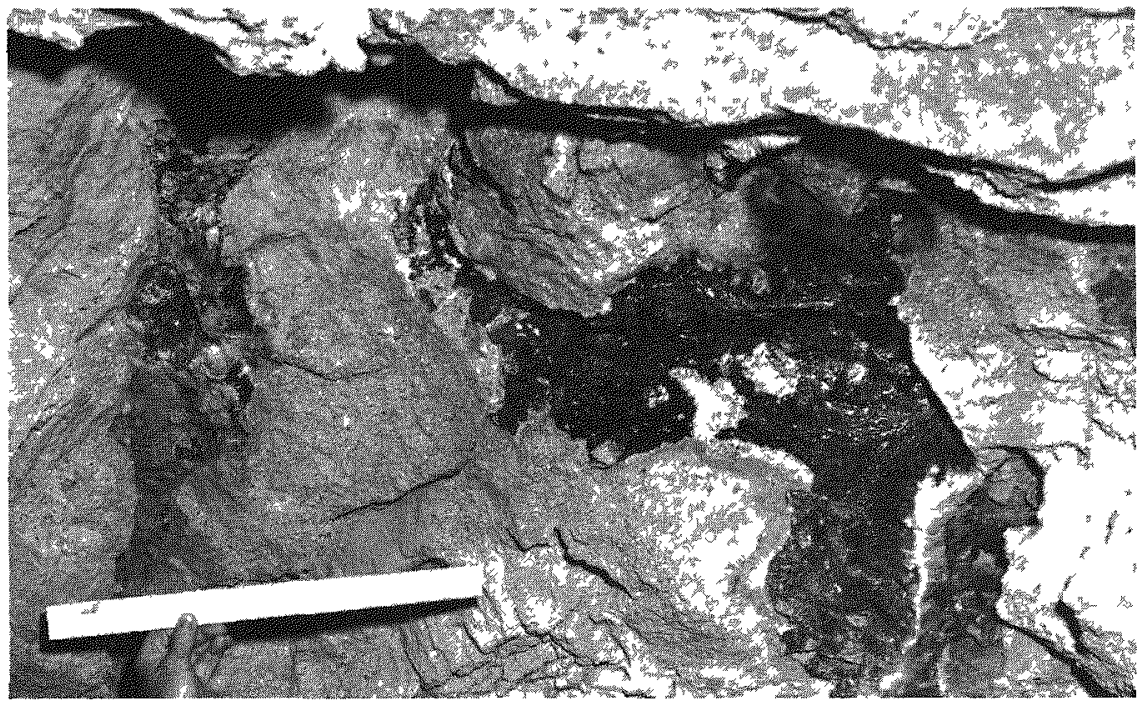

Fig. 3.14 Cracks sealed by resolidification of molten rock.

was taken in a tunnel dug in the vicinity of the cavity formed by an underground detonation. The darker areas were produced by the resolidification of molten rock forced into cracks. Filled cracks of this type have been found to extend into the surrounding rock as far as twice the stable cavity radius, that is, for several hundred feet around the explosion point.

If the hole, at the bottom of which the nuclear device is placed, is not properly stemmed and is intersected some distance above the explosion point by a fracture in the rock, a different situation could arise. The combination of poor stemming and the presence of a fracture could make it possible for gases and solid particles to vent from the expanding cavity.

The general conclusion drawn from experience with many nuclear tests is that, provided the emplacement hole is stemmed properly, faults and joints do not constitute significant vent paths. Nevertheless, it is the practice at present to avoid locating the nuclear 
device to be detonated on or immediately adjacent to a known fault. Geologic studies for the location of faults (see Chapter 4) are thus an essential aspect of safety analysis for underground nuclear explosions.

\section{SUMMARY}

An underground nuclear detonation is accompanied by the formation of a cavity as a result of vaporization of the rock surrounding the explosion point and the subsequent expansion of the vapor. The formation and initial rapid expansion of the vapor produce a sharp pressure wave (or shock wave) in the ground. As a general rule, if the detonation occurs at a sufficient depth, the roof of the cavity collapses after a time and as a consequence a tall column (or chimney) of broken rock and rubble extends upward.

Methods have been developed for calculating the radius of the cavity and for estimating the dimensions of the rubble chimney produced in underground nuclear detonations. The effects of the shock wave in the rock surrounding the explosion point can also be calculated provided the properties of the medium are known.

When the shock wave reaches the surface of the ground it is reflected back as a rarefaction wave. If the explosion is to be contained, and not break through the surface, the depth of burial must be such that, by the time the rarefaction wave is reflected back to the cavity, it is too weak to affect cavity growth.

To avoid seepage to the surface of noncondensable radioactive gases remaining after the nuclear explosion, the depth of burial must be appropriate to the energy yield. There is a small possibility of accidental venting of both gases and solid particles, but adequate closure (or stemming) of the hole or other opening leading to the underground detonation chamber should prevent such venting. Although faults and joints in the rock are not believed to constitute significant vent paths, efforts are made to avoid them. 


\title{
Chapter 4 \\ GEOLOGY, HYDROLOGY, AND SAFETY OF WATER SUPPLIES
}

\author{
INTRODUCTION
}

Geologic and hydrologic studies of sites to be used for underground nuclear detonations are intended to supply information primarily on the nature, arrangement, and special features of the rock formations in the area. The studies provide background information both for public safety and for the construction and stemming of the emplacement hole for the nuclear device (p.33). In Plowshare experiments for the recovery of natural resources, such as gas, oil, and minerals, for the creation of cavities to be used for underground storage, or for geothermal power generation (see Preface), the geology and hydrology of the region must, of course, be known in detail.

In addition to general background geology and hydrology, information is provided by the U. S. Geological Survey and others in three specific areas related to safety problems. One is concerned with the presence of faults through which radioactive matter might be released to the atmosphere. The second relates to geologic conditions that might lead to damage from ground shock and earth motion. Finally, situations are identified that could result in the abnormally rapid transport of contaminants in subsurface water, generally referred to as "groundwater."

An underground nuclear explosion always leaves some radioactive residues in the ground, consisting of fission products, tritium, 
and radioisotopes produced by the absorption of escaping neutrons. Most of this radioactivity is entrapped in the resolidified rock melt (p.39) at the bottom of the cavity and is not subject to transport by groundwater. The small proportion of the radioactivity remaining in soluble materials is, however, a potential source of water contamination to which consideration must be given.

Most high-yield nuclear tests are conducted at such depths that the detonation point is below the water table and lies within an aquifer, that is, in a rock formation through which groundwater moves. As a result, there will be some local contamination of the water. It will be seen in due course that entry of radioactive material into an aquifer and the subsequent movement away from the detonation point are both very slow. There is thus little or no danger of contaminated water emerging at wells or springs even within a few miles of the explosion site.

The essential purpose of hydrologic studies of the region in which an underground nuclear test is to be conducted is to identify existing ground (and surface) water systems and to determine where and how fast the water moves. The location for the nuclear detonation is then selected so as to ensure that radioactive contamination of useable water supplies is very unlikely to occur.

The geology and hydrology of the Nevada Test Site are well known as the result of numerous studies. When an underground nuclear detonation is to be conducted in a new area, in connection with the Plowshare program, for example, then use is made of the geologic and hydrologic data that have been accumulated over the years by the U.S. Geological Survey and by other organizations. If such information is not available or if greater detail is required for a particular test, it is obtained by appropriate studies.

\section{GEOLOGIC CONSIDERATIONS}

\section{Geology of Yucca Flat}

An example of geologic information developed as part of the safety program for underground nuclear detonations is given in Fig. 4.1. This diagram depicts the generalized geology of the Yucca Flat area of the Nevada Test Site (see Fig. 1.2) from a depth of 3000 feet below mean sea level to some 7000 feet above mean sea level. 
Most of the surface of Yucca Flat is alluvium (p.56), although other materials, which generally underlie the alluvium, are exposed in some places. The alluvium is underlain by several hundred feet of

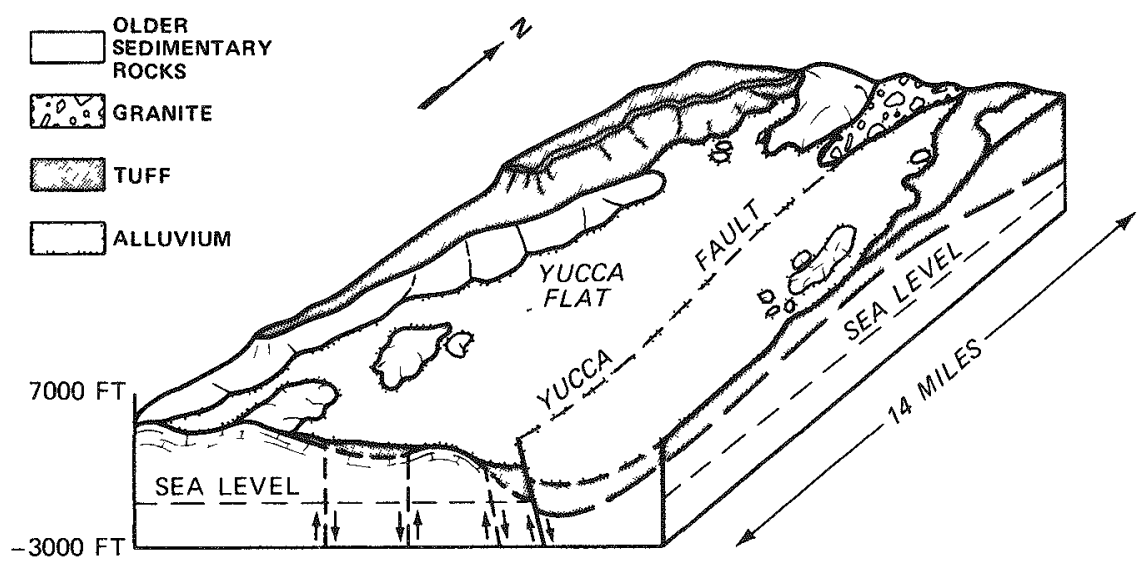

Fig. 4.1 Generalized geology of the Yucca Flat area of the Nevada Test Site.

bedded tuff (p.56) with varying physical characteristics. Below this are the older rocks consisting of carbonates (i.e., dolomite and limestone). At one place indicated at the north end of Fig. 4.1 there is an intrusion of granite.

\section{Observable and Concealed Faults}

Before proceeding to a further examination of Fig. 4.1, the geologic structure of Yucca Flat will be looked at in a little more detail. In the east-west section through the middle of the Flat, depicted in Fig. 4.2, attention is called to the feature marked Yucca Fault. A "fault" is a break or shear in the rock characterized by an observable or otherwise detectable displacement of one side relative to the other. A vertical displacement is seen clearly at the right of Fig. 4.2 (and also in Fig. 4.1). The arrows indicate the directions of displacement of the rocks.

The Yucca Fault is observable on the ground surface. There are in addition three inferred faults, also indicated by double arrows in Figs. 4.1 and 4.2, which are not seen on the surface in this area. In order to identify such concealed faults it might be necessary to carry out a detailed geological exploration. In some instances, however, a 


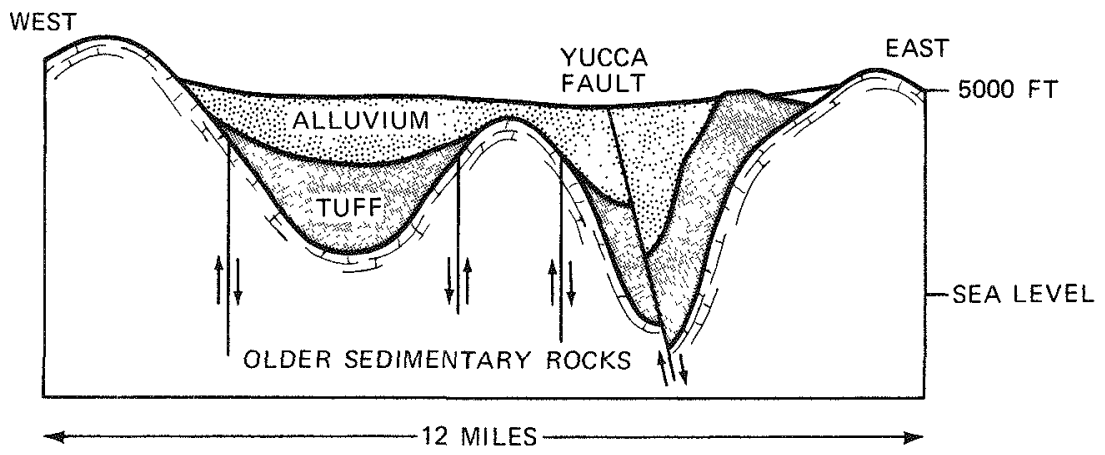

Fig. 4.2 East-west section through Yucca Flat showing observable and concealed faults.

fault which is not observed on the surface at one location is observable some distance away. The existence of concealed faults may occasionally be confirmed by underground nuclear detonations, because some of the surface fractures produced by the explosion tend to coincide with faults. This is illustrated in Fig. 4.3, which compares observed and inferred faults with surface fractures induced by four underground detonations, named Aardvark, Bandicoot, Bilby, and Haymaker.

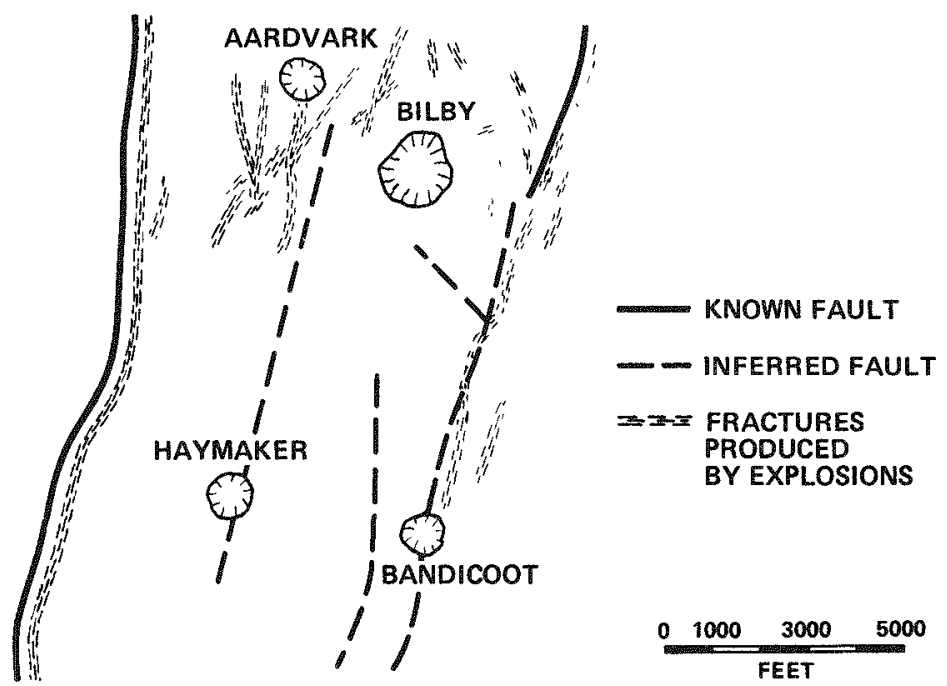

Fig. 4.3 Surface fractures produced in underground nuclear detonations and locations of faults. 
The low-yield Bandicoot event (October 1962), which may have been located on a hidden fault, was accompanied by the venting of radioactive matter. As mentioned in Chapter 3, venting through faults is believed to be improbable; however, pending further studies, it is the current practice to avoid locating an underground nuclear detonation in or near a fault. Every effort is therefore made to determine the positions of faults in advance of a test.

\section{Effects of the Geologic Environment}

The importance of the geologic environment of the explosion point may be illustrated by means of Fig. 4.1. Suppose, for example, a nuclear device were detonated underground at about the center of the granite mass at the north end of Yucca Flat. To the east, the seismic energy would propagate outward initially through a substantial layer of alluvium and tuff. To the west, on the other hand, the energy would move through the more dense carbonate rocks with a higher velocity. It is probable therefore that seismic effects such as ground motion would be more apparent in one direction than in the other.

A somewhat similar situation can be envisaged from Fig. 4.2. Consider an underground detonation in a deep hole somewhat west of the main fault in central Yucca Flat. To the east, the seismic energy would first have to traverse a region of alluvium and tuff, whereas to the west it would soon enter the buried ridge of carbonate rocks. Again, the effects might be different in opposite directions.

It is clear, therefore, that geologic knowledge of the area of the detonation site is required in order to properly evaluate and predict ground motion effects. Some of this information may be available from existing sources when a nuclear device is to be detonated underground in any new area. But frequently it must be obtained from special studies in preparation for a particular test. More will be said in Chapter 5 about the observation and the prediction of ground motion. The effects on structures will also be considered in that chapter.

\section{HYDROLOGIC CONSIDERATIONS}

\section{Objectives of Hydrologic Studies}

Broadly speaking, hydrologic and geologic studies in support of public safety in underground nuclear detonations provide informa- 
tion on the occurrence, movement, quality, and quantity of ground and surface waters that might be affected by the explosion. Hydrologic considerations are also important in locating the site and drilling the hole in which the nuclear device is to be detonated.

The hydrologic data obtained from exploratory drill holes include the location of aquifers, transmission rates for groundwater, and direction of flow. Further studies are then made to determine whether or not there will be radioactive contaminants introduced into the groundwater by the nuclear detonation. Should some contamination of the water be expected, predictions are made as to the path it will take away from the detonation site. These predictions are used to prevent the inadvertent use of this water.

The investigations carried out by the U. S. Geological Survey and others in relation to the safety of water supplies fall into three main categories, namely, regional, site, and special studies. These will be considered in turn.

\section{Regional Studies: The Nevada Test Site}

The principal aims of the regional studies are to define the main water-bearing rock formations (i.e., the aquifers), to determine the movement patterns of groundwaters through the aquifers to the discharge areas, and to follow the changes in mineral content of the groundwater as it moves through various rocks. Stream discharges and the yields of wells and springs are recorded both before and after the nuclear explosion to determine if there have been any changes.

The level of the water table is measured (and other geologic and hydrologic information is obtained) by drilling deep exploratory holes at several places, if necessary. As a general rule, the water table does not run parallel to the surface. Below Yucca Flat, however, the water table is essentially flat at about 2400 feet above mean sea level; this is some 1600 feet below the lowest surface areas of Yucca Flat.

For a nuclear detonation at a depth of less than 1600 feet, as is frequently the case at Yucca Flat, the explosion point will be above the water table. Hence, water contaminated by radioactive residues can reach an aquifer only by migrating downward. Downward migration is slow although it can increase periodically from rainfall and other precipitation. Since the precipitation at the Nevada Test Site is small, very little surface water penetrates to the groundwater level. 
Under the exposed ridges to the east and west of Yucca Flat and also under the buried north-south ridge in the central region the main body of water is in the carbonate rocks. These rocks are characterized by numerous fractures. Because of the fractures, the carbonate rocks transmit water more readily than do alluvium and the softer volcanic rocks. Therefore, groundwater from a considerable part of the Nevada Test Site moves through the carbonate rocks and they largely determine the migration of this water.

Information on the direction of movement of groundwater under the Nevada Test Site region is obtained from several different types of observations. These include determinations of the level of the water table and chemical analyses of the groundwater and of the discharges at springs or wells at various locations. Clues are also provided by the geologic structure of the area. However, the groundwater flow system has no connection with and differs markedly from the drainage patterns of surface water at the Nevada Test Site.

\section{Hydrology in Vicinity of the Nevada Test Site}

The present interpretation of the hydrologic setting in the vicinity of the Nevada Test Site is shown in Fig. 4.4; although the general features are essentially correct, additions and refinements are made from time to time as more data from continued studies become available. Many of the surface drainage basins have no outlets for surface water, thus confirming that the transport patterns of the surface and ground waters are not related.

As indicated by the arrows, groundwater from deep below Yucca Flat flows southward under the Nevada Test Site. The movement to the east and west is greatly retarded by underground ridges of quartzite and shale, which are relatively impervious to water. Figure 4.4 also shows that the level of the water table falls from north to south. It was mentioned earlier that most of the water movement occurs in the highly permeable carbonate rocks.

After passing under the Mercury area, the groundwater moves in a southwesterly direction and is probably discharged at springs in the Amargosa Desert, roughly 50 miles from Yucca Flat. This is the place where most of the water that might contain radioactivity from underground nuclear explosions at the Nevada Test Site would be expected to appear. But by the time the water reaches the discharge area, the radioactivity remaining would be insignificant (p.81). 


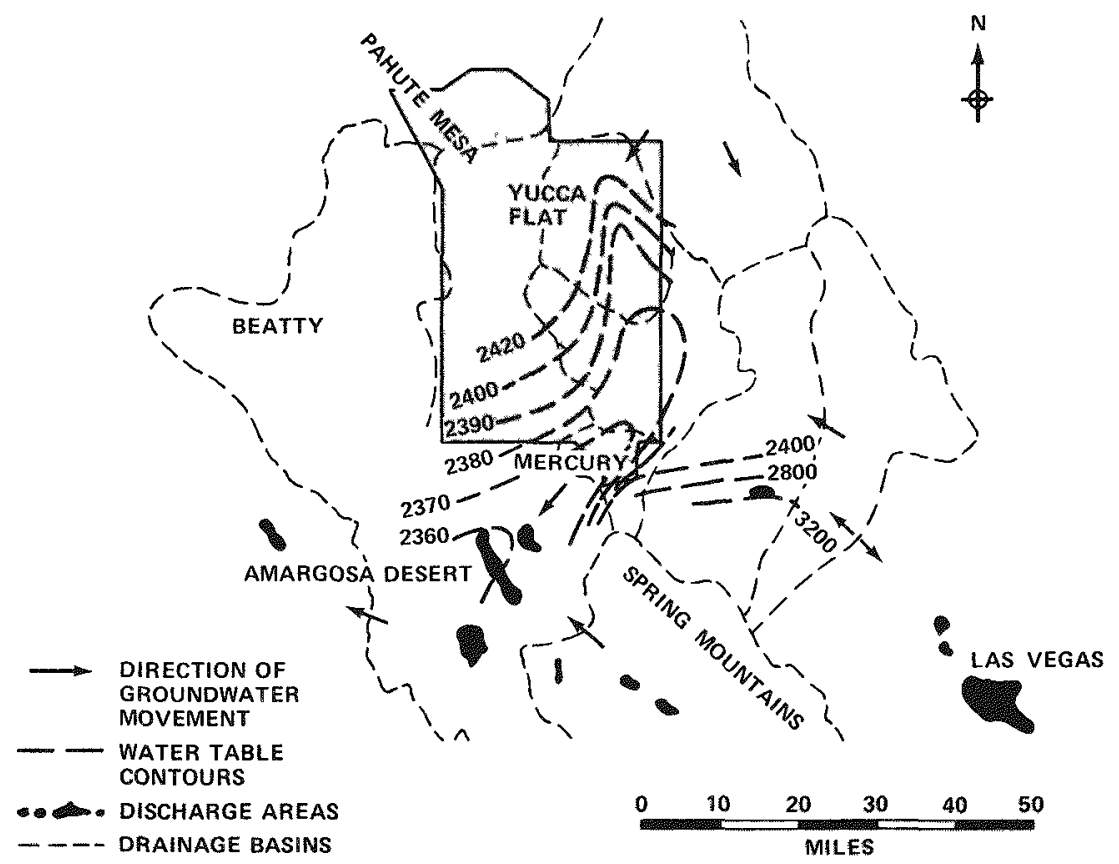

Fig. 4.4 Hydrologic setting in the vicinity of the Nevada Test Site.

The rate of movement of groundwater southward through the carbonate aquifers below Yucca Flat and beyond has been calculated from the known difference in level of the water table and the probable permeability of the rock. The rate of transport obtained in this manner for the carbonate aquifers ranges from 7.3 to 730 feet per year, depending on the assumed porosity of the rock. The rate of movement (or transport) of water in an aquifer is often referred to as a "flow" rate. It must be understood, however, that the water in an aquifer does not "flow" in the strict sense of the term. The water actually seeps through pores, cracks, crevices, and other spaces in and between the rock formation. This movement is consequently a slow process.

Even if the rate of movement of the groundwater is taken to be the maximum calculated value of 730 feet per year, it would require about 360 years for groundwater from Yucca Flat to reach the natural discharge areas. It would be considerably longer if the flow rate is less than 730 feet per year, as it probably is.

A knowledge of the transit time of the groundwater from Yucca Flat to the Amargosa Desert springs is important. Since the value 
given above, based on a calculated transport rate, is subject to uncertainty, efforts are being made to determine the transit time by direct observation. One way in which this can be done is to evaluate the permeability of the aquifer from the rate at which water can be pumped from it. This is combined with the known difference in water levels and an assumed porosity to calculate the rate of movement of the water. Another approach is to measure the amounts of natural radioactive isotopes, such as carbon-14 or tritium, at different points along the flow pattern of the water. From the known half-lives of these isotopes, it is possible to determine the time taken for the water to travel between the various places.

The data obtained so far by isotopic analysis indicate that the flow rate of water beneath Yucca Flat is roughly 7 feet per year. This is about the same as the minimum calculated value. The transit time of groundwater to the discharge area would then be something like 38,000 years, apart from special circumstances which might tend to speed up the flow locally, such as passage through a region of exceptionally high permeability.

It should be noted that the rate of flow of groundwater away from the explosion point is the maximum rate at which radioisotopes can travel in the water system. Most of the contaminating material actually moves much more slowly than the transporting water (p.73). Tritium as tritiated water, however, is an exception and travels at essentially the same rate as the groundwater.

\section{Hydrology of Central Nevada}

The hydrology of the Nevada Test Site area has been described in some detail because the region is the one in which most underground detonations are performed. Similar hydrologic studies have been made in Central Nevada where one nuclear device has been exploded in a calibration test (p.2).

The conditions in Central Nevada are different from those at the Nevada Test Site. There is more rain at the former location and perennial streams originate in the highlands but sink into the ground at the foot of the mountains. The water table in the valleys is then generally less than 600 feet below the land surface. In many of the valleys the water table is actually above the lowest places so that surface waters are present.

From studies of the water table levels and of the regional geology, indications have been obtained of the groundwater flow 
pattern. It appears that part of the water beneath Hot Creek Valley, where the underground detonation was carried out, will eventually reach discharge areas in the adjacent Railroad Valley. The crowflight distance between these valleys is about 40 miles, but the groundwater has to follow a much longer path through alluvium. The transit time between the explosion point and the discharge area is thus calculated to be several hundred years.

\section{Hydrology of Amchitka Island}

Beneath Amchitka Island, Alaska, which was selected for testing nuclear devices of high yield (p. 4), the water table is quite shallow. Furthermore, there is sufficient precipitation to maintain numerous lakes and small streams on the surface of the island. The rock underground in the vicinity of the test locations, however, has a very low permeability. Several faults through which water could possibly penetrate the rocks are known to exist, but the holes for the placement of the nuclear devices are drilled at locations where there are no detectable faults. On the basis of all available information, it is expected that transport by groundwater of the radioactive residues of an underground nuclear explosion at Amchitka Island will be extremely slow.

\section{Site Hydrology Studies}

Site hydrology, the second category of hydrologic studies, involves testing exploratory drill holes close to the proposed location of the nuclear device to be detonated. The water yielding potential is measured at various depths and samples of water and rock are collected for chemical analysis and identification.

In preparation for the Rulison gas-stimulation experiment near Grand Valley in western Colorado (September 1969), an exploratory drill hole indicated the geologic structure shown in Fig. 4.5. After a steel casing was cemented into this hole, the casing was perforated at various levels below 6000 feet in order to study the water potential. Small amounts of water were recovered but their analysis showed that they originated mostly from the drilling and cementing operations. Little or no free-moving water was found in the Mesaverde Shale/Sandstone, where the explosion point was located; this was apparently also true for the Ohio Creek Conglomerate immediately above it. 


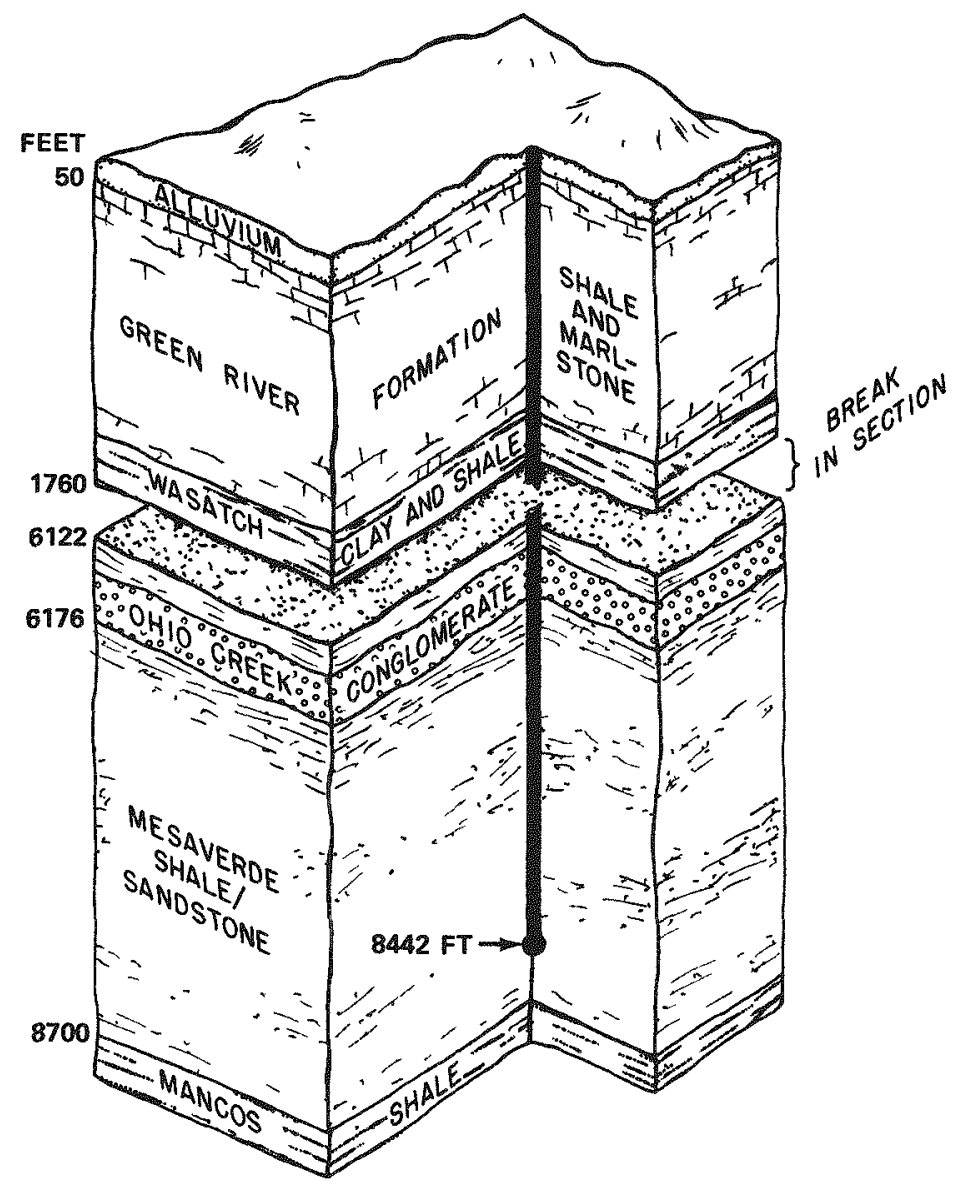

Fig. 4.5 Geologic structure of the Rulison site in Colorado.

Collection of groundwater samples, to be tested for certain natural radioactive isotopes, such as carbon-14 and tritium, traces of which are normally present in water, is part of the site hydrology program. The information so obtained is used in determining the rate of flow of groundwater (p.67). Chemical analysis is also utilized, as previously mentioned, to indicate the path of the groundwater.

\section{Special Hydrologic Studies}

At the present time, the special hydrologic studies are of two main types. One is to observe the changes in the water systems 
resulting from the effects on the rocks surrounding an underground nuclear detonation. For example, wells and pumping systems are examined for possible damage. In this regard, it may be mentioned that the water levels of wells in the vicinity of the Nevada Test Site have been found to vary by no more than an inch or so even after the largest underground explosions.

The second kind of hydrologic investigation that falls in the area of special studies is aimed at obtaining specific information about the character and extent of fractures in the carbonate rocks that underlie both the Nevada Test Site and the Central Nevada area. It is hoped in this manner to obtain better data on the rate of travel of the groundwater.

A procedure used in these studies is to add to the water a trace of an easily detectable substance, which is normally not present in the water. The material is introduced into the water at one point in an aquifer and the times of its arrival at various points at a distance are noted. Proposed substances for use in such investigations are potassium chloride, tritium (as tritiated water) or other radioactive isotope in harmless quantities, and a rhodamine (red) dye.

\section{MIGRATION OF RADIOACTIVE ISOTOPES IN GROUNDWATER}

\section{Possibility of Radioactive Contamination}

The discussion so far has referred to what has been (and is being) done to understand the behavior of water systems in and around the place where a nuclear device is to be detonated underground. The possibility of contamination of groundwater by radioactivity has been touched on briefly from time to time. Now this problem must be considered in more detail, since one of the basic purposes of the hydrologic (and related) studies is to identify and keep track of any contaminated water in both space and time.

Because of the slow movement of the groundwater, no contamination should reach any possible discharge area where water is used in Nevada for more than a hundred years after an underground detonation. In these circumstances, the original radioactivity will have decayed to such an extent that the water would be safe for human consumption. It is of interest to note that after fourteen years of underground testing of nuclear devices, no radioactivity above the 
normal background has been detected at springs or wells in Nevada (or elsewhere).

\section{Prediction of Radioisotope Migration}

The major part of the program for studying contamination of groundwater is concerned with the prediction of the migration rate and path of radioactive isotopes that may be transferred in the water. This program is conducted by Teledyne Isotopes, Inc., under contract to the U.S. Atomic Energy Commission. Most of the radioactive residues from an underground nuclear detonation are present in the glass-like material formed by resolidification of the molten rock which collects at the bottom of the cavity. This material dissolves very slowly in water and it probably contributes very little to the overall radioactive contamination. The chief potential sources of contamination of groundwater, representing a small fraction of the total radioactivity, are present in different forms.

The gaseous (noncondensable) isotopes krypton-90 and xenon137, formed in fission, have very short half-lives. They consequently decay within the chimney to form strontium-90 (by way of short-lived rubidium-90) and cesium-137, respectively. These decay products, which have relatively long half-lives (about 28 to 30 years), are deposited as very fine solid particles on rock (rubble) surfaces and also admixed with the rock dust formed by grinding action during chimney collapse. If groundwater enters the chimney, soluble compounds of strontium and cesium would be dissolved off the rock surfaces and leached from the rock dust. Some isotopes of iodine and ruthenium that are initially present in the noncondensable vapor may also solidify in the chimney when it cools. These isotopes and most of their decay products have such short half-lives that thay will have essentially disappeared before water starts to move out of the chimney.

Nuclear devices of high yield are detonated at great depths in order to prevent any release of fadioactivity to the atmosphere. There is then a possibility that part of the rubble chimney will be within an aquifer. The water entering the chimney would then become contaminated with radioactivity. The manner in which this can occur will be examined by considering a relatively simple situation.

Suppose the nuclear device is detonated below the level of the water table, but the rubble chimney extends above the water table. 
The first thing that happens after the formation of the chimney is that the surrounding water begins to flow into the chimney. Measurements in holes drilled in the chimney show the initial water levels to be below that in the surrounding area (Fig. 4.6). The chimney is thus said to constitute a "sink" into which the groundwater tends to move. The water movement is quite slow and it may take several years to fill the chimney to the level of the water table.

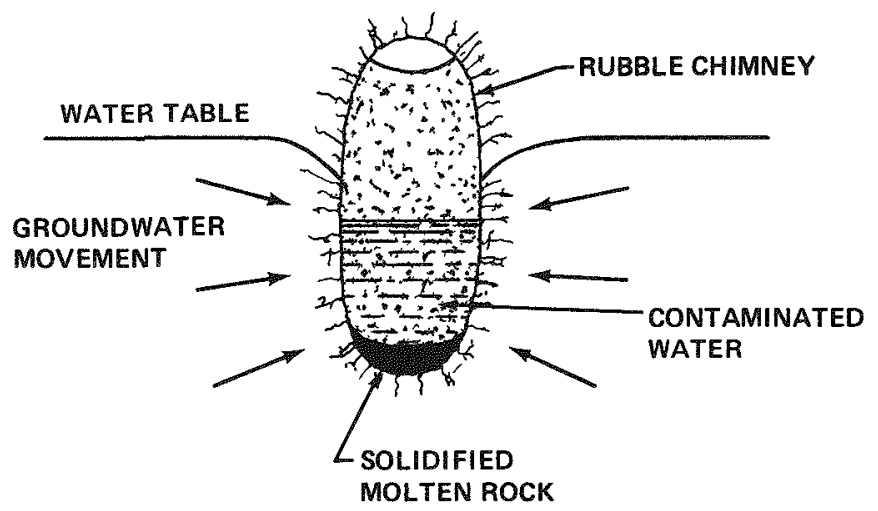

Fig. 4.6 Water entering the chimney formed by an underground explosion in an aquifer.

Water in the chimney will gradually dissolve some of the material containing radioactive isotopes. In addition, tritiated water will be present. In due time, the level of the water in the chimney rises and then the groundwater, including the contaminated water in the chimney, will resume its normal direction of transport. Fresh water will enter the chimney and the water moving out of the chimney will be contaminated (Fig. 4.7).

In considering the fate of the radioisotopes being transported, it is important to remember that the groundwater moves through pores, cracks, and interstices within the rock material (p.66). In this environment there is a continuous exchange of radioactive materials between the moving water and the rock surfaces. The behavior of tritium in water, however, is different as will be explained later.

\section{Sorption and Dispersion}

As the contaminated water moves into clean (uncontaminated) rock outside the chimney, the rock surface takes up much of the 


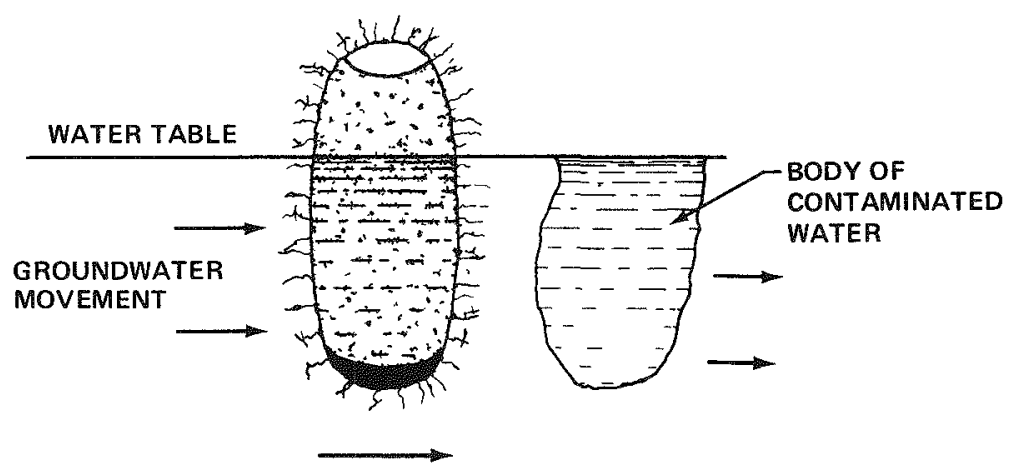

Fig. 4.7 Body of significantly contaminated water leaving the chimney (hypothetical).

radioactivity (other than that due to tritium) from the water. This process, called "sorption," tends to slow down the transmission of the radioactive contamination relative to the flow of the groundwater.*

Much of the radioactivity held by the rock surface (i.e., sorption) is later removed (i.e., desorption) by relatively uncontaminated groundwater. But the net effect is to extend the period of time available for the radioactivity to decay before the groundwater reaches a well or a spring.

Another factor that has the effect of reducing the concentration $\dagger$ of radioactive isotopes in the groundwater to some extent is "dispersion," that is, the mixing that occurs between contaminated water and uncontaminated water at the boundary where they meet. Because of this mixing, the radioactivity in the water is diluted and the concentration of the contaminating material is decreased. As a consequence of dispersion at the front of the contaminated water as it moves in the general flow direction, a very low concentration of radioactive material precedes the main body of water and indicates that larger concentrations may be on the way.

*Chemists use the word "sorption" as a general (or catchall) term to cover the different ways in which a solid can remove dissolved substances from a solution.

tThe concentration of any substance in a solution is expressed as the quantity (or mass) of the substance divided by the volume of water (or of solution) in which it is dissolved. In other words, the concentration is the quantity (or mass) of the substance in a unit volume of water (or of solution). 
It was seen in Chapter 2 that every radioactive isotope decays continuously and, hence, decreases in amount at a rate dependent on the half-life of the particular isotope. Calculations show that, from natural decay alone, the radioactivity of the more important residues of a nuclear detonation will become insignificant after 10 to 15 half-lives, depending on the total amount of radioactive material initially entering the groundwater. At the end of this period the activity will have decreased to less than one-thousandth (i.e., less than 0.1 percent) of its original value (p.15).

\section{Water Flow Pattern Through Chimney}

The first step in predicting the way in which radioactive isotopes produced in an underground nuclear detonation migrate with the groundwater is to consider the motion of the groundwater. The original radioactive water occupies the rubble chimney, but as this water moves into the aquifer it will tend to spread out. The reason is that the permeability of the surrounding rock is less than it is in the chimney.

A mathematical analysis has been made of the "flow" lines from a region of relatively low permeability, representing the original rock medium, into one of higher permeability, that is, the rubble chimney, and then out again into a medium of lower permeability. A horizontal cross section through the pattern obtained in this manner is given in Fig. 4.8.

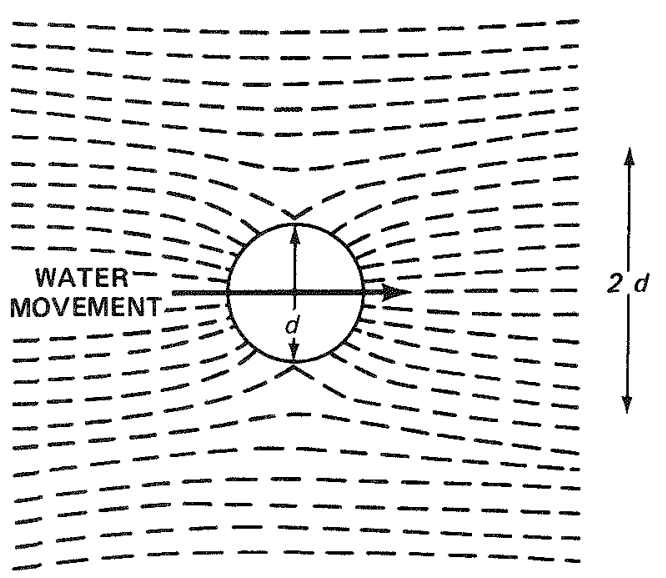

Fig. 4.8 Horizontal cross section of water pattern into and out of a chimney. 
The flow lines in the normal aquifer are seen to be drawn in as they approach the rubble chimney with its higher permeability. After leaving the chimney, the flow lines of contaminated water spread out again. The calculations indicate that if the permeability of the rubble is considerably higher than that of the surrounding rock, the width of the body of contaminated water that is eventually formed is roughly twice the width of the chimney.

It was implied on page 48 that the diameter of the rubble chimney is about the same as that of the stable explosion cavity. Consequently, the width of the body of radioactive water is approximately twice the diameter of the cavity. If the latter is $d$, the width of the contaminated water is $2 d$, as shown in Fig. 4.8.

A body of contaminated water of this width will then travel at the normal rate at which the groundwater moves in the aquifer. Since the flow rate is not known accurately (p.67), there is an uncertainty in predicting the migration of radioactivity in groundwater. Consequently, for purposes of calculation, the rate of movement is assumed to be fairly high, probably much higher than the actual rate. The results obtained then include a substantial margin of safety.

\section{Distribution Coefficients and Sorption}

The next stage in the calculation is concerned with the effects of sorption (and desorption), that is, the processes whereby the rock removes (and later gives up) some of the radioactive material present in the contaminated water. Allowance for sorption is made by means of a number called the "distribution coefficient." It is a measure of the way in which a particular isotope is distributed (or apportioned) between the solid rock material and the contaminated groundwater. If the distribution coefficient is large, much of the isotope is taken up by the solid, but if it is small most will remain in the water.

The value of the distribution coefficient for a given element depends primarily on (1) the character of the aquifer rock material and (2) the chemical form in which the element is present in the water. The temperature, pressure, and other dissolved substances in the water also have some influence. The distribution coefficient is a measurable quantity and, ideally, the measurements should be made in the aquifer. Since this would be difficult, it is the general practice to determine distribution coefficients in the laboratory using rock and water collected from the aquifer of interest. 


\section{Effect of Dispersion}

As a result of dispersion, the radioactive material (including tritium) is spread through an increasingly larger volume of water in the aquifer. Consequently, the concentration of radioactivity in the water is reduced. The situation may be illustrated by the diagram in Fig. 4.9. The full line represents the variation in radioactivity along

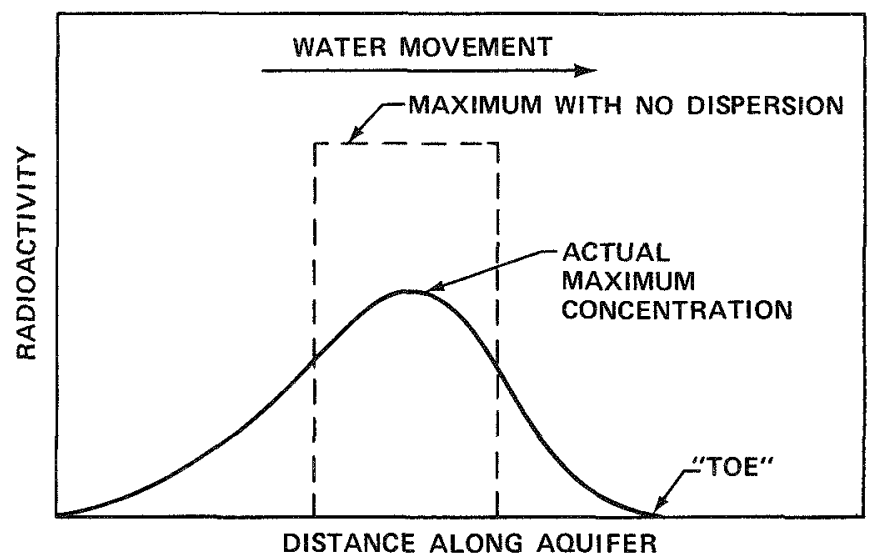

Fig. 4.9 Effect of dispersion on the concentration of radioactivity.

the length of the body of contaminated water as a result of dispersion, whereas the broken lines show what it would have been in the absence of dispersion.

Two effects of dispersion are apparent from Fig. 4.9. In the first place, the maximum concentration of radioactivity in the water, corresponding to the peak (or maximum) of the curve, is considerably less than if there had been no dispersion.

As a general rule, this particular effect of dispersion is more important when the diameter of the body of contaminated water is relatively small. This might be the case, for example, with a detonation of very low yield for which the cavity and rubble chimney would have a small diameter.

The second effect of dispersion is one which was referred to on page 73. It is seen from Fig. 4.9 that the right end (or "toe") of the curve extends some distance in front of the main body of contamination. Furthermore, the radioactivity concentration in the toe region is quite small. In other words, as a consequence of 
dispersion, water containing minute traces of radioactive materials, which can nevertheless be detected, will arrive at the discharge area much earlier than the bulk of the contamination. Thus, dispersion would provide an early and harmless warning in ample time for precautionary measures to be taken if necessary.

\section{Combined Effects of Sorption, Dispersion, and Decay}

An equation has been developed that expresses in mathematical terms the shape of a curve of the type in Fig. 4.9 for each individual isotope. Thus, the equation gives the concentration of the isotope in groundwater at any time after the contaminated water starts to leave the rubble chimney formed by the nuclear explosion. The rate of movement of the water obtained by standard hydrological methods is used for the calculations. Hence, the location of the radioactive contamination in the aquifer corresponding to any specified time can be determined.

The equation referred to above takes into account (1) the distribution coefficient, to allow for the effect of sorption, (2) the dispersion coefficient, and (3) natural decay of the radioactivity. By solving the equation, it is possible to predict the location and concentration of a given radioisotope in the groundwater at any time after an underground nuclear detonation in an aquifer.

The dispersion considered so far is in the direction of movement of the underground water; this is, in fact, the most important kind of dispersion of the radioactive material. There is, however, also some dispersion in both horizontal and vertical directions, at right angles to the main movement of the water. Allowance can be made in the calculations for these situations.

In carrying out a detailed computation of the migration of radioactive isotopes, it is necessary to know how much of each isotope is produced in the nuclear detonation. For illustrative purposes, however, it is sufficient to consider only two isotopes, namely, strontium-90 and tritium. From the standpoint of water consumption, strontium-90 is potentially the most hazardous product of fission and its behavior in groundwater may be taken to be characteristic of other fission products. Tritium, on the other hand, is the most significant radioisotope generated in a device in which the explosive energy is derived mainly from fusion reactions. Actually, all types of nuclear explosions will produce strontium-90 and 
tritium, although in different proportions. But since these two radioactive isotopes behave quite differently in an aquifer, it is convenient to consider them separately.

\section{Effect of Detonation Energy Yield}

First, something must be said about the effect of the energy yield of the explosion. Surprisingly, the yield is not of as much consequence as might appear at first sight, provided the ratio of fission energy to fusion energy is the same in every case. Although the total quantity of a given isotope produced is important, it is the concentration (i.e., the amount per unit volume) in the water that best characterizes the biological significance of the radioactive contamination.

The total quantity of any particular isotope formed in a nuclear detonation increases with the energy yield of the device (for a given fission to fusion ratio), but the volume of the chimney also increases to roughly the same extent. Hence, the original concentration of the isotope in the water present in the chimney is always about the same, regardless of the total yield. These arguments are valid provided the scaled depth of burial of the nuclear device, the surrounding rock, and other local factors remain unchanged.

\section{Migration of Strontium}

Laboratory experiments indicate that strontium is strongly sorbed by many rock surfaces; thus, the distribution coefficient is usually high and it will be the same for all the isotopes of strontium. Calculations, based on a reasonable value for the distribution coefficient, show that, as a result of sorption (and desorption), the migration of strontium-90 along an aquifer would be very slow, much slower than the rate of movement of the water. Consequently, by the time the contamination could reach a discharge area (some 50 miles away from Yucca Flat at the Nevada Test Site), normal decay would have reduced the concentration of the strontium-90 considerably below that at which it could constitute a hazard to people drinking the water.

The other important radioactive isotope remaining in the chimney after a fission explosion is cesium-137. This is also strongly sorbed and is expected to behave in the same way as strontium-90. In 
view of the marked effect of sorption for these two isotopes, dispersion does not need to be considered. The prediction is then that the fission product residues from the detonation of a nuclear device at the Nevada Test Site will cause no significant radioactive contamination of groundwater at a discharge area. The same situation would be required before any other location would be used for an underground detonation.

\section{Migration of Tritium}

For tritium, the circumstances are somewhat different; the sorption effect appears to be quite small and so it can be neglected in the migration calculations. The important factors are then dispersion and natural decay. An illustration of the predicted transport of tritium based on these calculations is given in Fig. 4.10. For this illustration, the rate of movement of the groundwater was taken to be 330 feet per year. The assumed direction of migration, in a southerly and somewhat westerly direction, is fairly typical of the

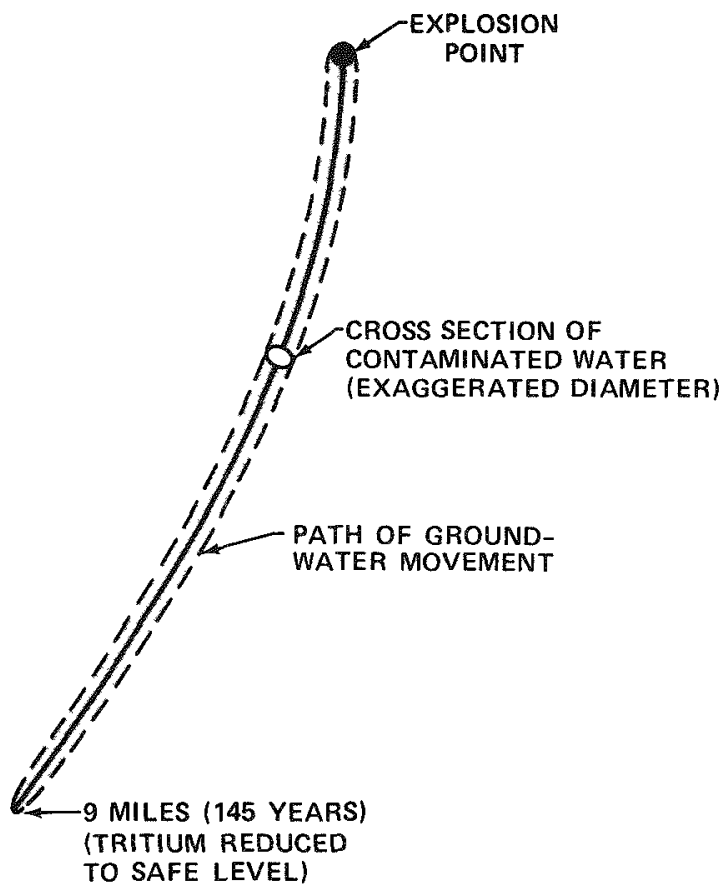

Fig. 4.10 Predicted transport of tritium in an aquifer based on a water movement of 330 feet per year. 
Nevada Test Site. The path of the water containing tritium, as depicted in Fig. 4.10, is in two dimensions in horizontal directions. There is, of course, a third (vertical) dimension that is not shown.

The water contaminated with tritium spreads out to a width approximately twice the diameter of the rubble chimney soon after it leaves the explosion zone. Then, some 145 years later, according to the calculation, dispersion and decay will have decreased the tritium to a concentration which is below that acceptable for continuous consumption of the water. During the 145 years, at the assumed rate of movement of 330 feet per year, the tritium will have traveled a total distance of about 9 miles from the explosion point. Since the half-life of tritium is a little more than 12 years, the migration has extended over some twelve half-lives. Normal radioactive decay would thus account for most of the decrease in the activity.

There is one point to which attention should be called because it is not apparent from Fig. 4.10. The map shows the whole region through which tritium will have moved at some time during the 145 years following the detonation. It must be understood, however, that only part of the water in that region is contaminated at any one time. The bulk of the tritium, although somewhat dispersed, moves forward with the same speed as the water in the aquifer, leaving almost uncontaminated water behind it. It takes only a few years for nearly all the tritium present in a body of water to pass any point in its path.

The calculated time of 145 years and travel distance of 9 miles given above are based on an assumed migration rate of 330 feet per year for the groundwater. In most cases, the water moves more slowly. The distance traveled by the tritium before the amount is of no significance in drinking water would then be much less than 9 miles. Thus, it appears that the tritium produced in an underground nuclear detonation would not be a problem as far as water supplies are concerned.

\section{Multiple Detonations}

The general conclusion to be drawn is that, by taking normal precautions, there is essentially no danger that groundwater contaminated with any significant amount of radioactivity will reach a discharge area in the vicinity of a single detonation, no matter what the explosive energy yield. The qualification that this applies to a 
single detonation is important. If there were several detonations at short intervals in the same general location in an aquifer, it is possible that water contaminated by the radioactive residues from one explosion would migrate into the chimney formed in another explosion, and so on. The concentration of strontium-90 and of tritium in a given quantity of water would then be greater than for a single detonation.

Although it is not at all probable, such a situation could conceivably arise at the Nevada Test Site. At a water migration rate as high as 730 feet per year, it would take about 370 years for water to reach the Amargosa Desert springs, the nearest discharge points, some 50 miles from Yucca Flat. The only important radioactive contaminant would be tritium, but the amount remaining, even from several hundred fusion devices, would still not be a hazard to people drinking the water regularly.

There is a possibility that a large number of nuclear devices might be exploded at short intervals in a natural gas field. In this event, the location would be chosen such that there would be no significant contamination of useable water supplies.

\section{SUMMARY}

Studies of geology and hydrology are important aspects of the program for ensuring safety in underground nuclear detonations. The geology of the explosion site and also of the surrounding area affects the propagation of seismic waves that can cause ground motions at a distance from the explosion. Fault locations are identified so that they can be avoided in the placement of the nuclear explosive device.

The main objective of the hydrologic studies is to provide the background information required to predict where groundwater that might be contaminated with radioactivity will be at all times after an underground detonation. Calculations show that only insignificant concentrations of potentially hazardous radioactive isotopes, particularly tritium and strontium-90, are expected at places where groundwater is used, that is, at springs and wells. If unexpectedly large amounts of radioactivity should enter the groundwater, its presence would be detected long before it became a hazard. Appropriate action would then be taken to prevent use of the contaminated water. 


\section{Chapter 5}

\section{GROUND MOTION \\ AND THE RESPONSE OF STRUCTURES}

\section{GENERAL CONSIDERATIONS}

\section{Introduction}

In Chapter 3 (p.36) it was stated that, at some distance from an underground nuclear detonation, the shock wave in the ground weakens and becomes an elastic (or seismic) wave. Such a wave causes the surface of the earth to vibrate or undulate, up and down, to and fro, and side to side, just as does an earthquake. Hence, buildings at considerable distances, possibly over a hundred miles away from the explosion point, might conceivably suffer some damage from an explosion of high energy yield. Studies of ground motion accompanying underground nuclear detonations and of the response of various structures to this motion are thus an essential aspect of the safety program. From such studies methods have been developed for predicting how structures of different types may be expected to respond to a particular detonation.

More than three hundred announced underground nuclear detonations have been conducted in Nevada, but there has been no structural damage to any high-rise building in Las Vegas, the nearest large city (see map in Fig. 1.1). As will be shown in due course, buildings of this type are the most susceptible to damage from an underground detonation at a distance. Motion of the ground and of buildings has been detected by instruments and by people in Las 
Vegas (and other places), but the extent of the motion was too small to cause anything but minor nonstructural damage.* Similar damage has been experienced from tests in other areas and this will be described later in the chapter.

It is of interest to note that human beings can detect motion much below the level at which even minor damage to buildings can occur. A rough comparison, in the form of rungs of a "threshold ladder," is made in Fig. 5.1. The lowest rung indicates the minimum (or threshold) motion that can be detected (and measured) by a

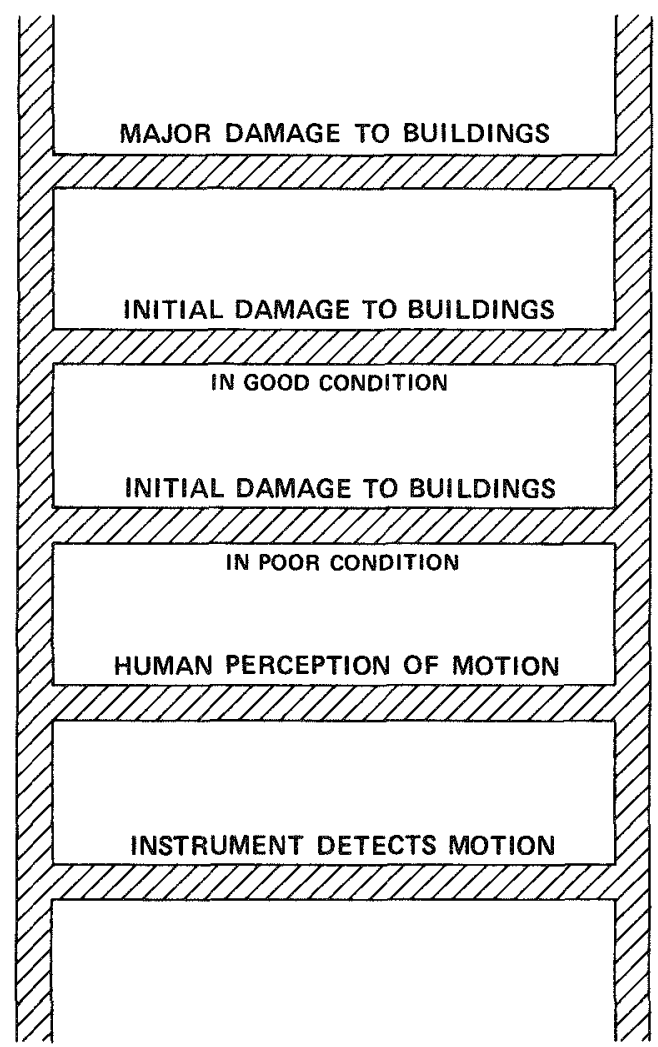

Fig. 5.1 Qualitative representation of relative sensitivities to ground motion.

* Nonstructural damage may consist, for example, of cracking of plaster and stucco, slight damage to panels and interior walls, and separation at joints in masonry (brick, block, or stone) walls. Such damage does not affect the use of the building. 
suitable instrument. The next rung represents the level at which human perception of motion would begin. Initial damage to buildings, even those in poor condition, would require a much larger ground movement.

The relative distances between the rungs in Fig. 5.1 must not be taken to have any precise numerical significance. The important point to be made is that there is a great difference between the motion level at which people would be conscious of movement in buildings (or of the ground) and that at which damage would begin even in a relatively weak structure.

Buildings that look alike can actually be very different in their ability to withstand motion. The variation in the perception of motion for different people is also quite large. Consequently, each rung of the threshold ladder in Fig. 5.1 is actually a broad band representing a range of motion levels. Nevertheless, the conclusion of the preceding paragraph is generally true. Even a person with poor perception of motion would be able to detect motion well before it reached the level at which a building would suffer even minor damage.

\section{Factors Affecting Ground Motion}

The ground motion recorded by an instrument at some distance from an underground nuclear detonation is the net result of a number of complex processes. As the seismic wave produced by the explosion travels through the ground, it encounters many major and minor geological discontinuities or boundaries. At each such discontinuity, the wave may be transformed into several other waves with different travel paths and characteristics. The energy may be partly reflected and partly refracted; that is to say, part of the seismic wave may be turned back and part may be bent somewhat away from its original direction of travel, as indicated in Fig. 5.2. As a result of these and other physical phenomena, there are many different transmission paths for the seismic energy. Consequently, the seismic disturbance at a distance from the explosion is generally a complex combination of waves of different types reaching a given point by several different paths (Fig. 5.3).

The detailed characteristics of the ground motion depend on many factors. The most important are the energy yield of the nuclear device, the depth of burial, the distance from the explosion point, 
and the nature of the ground where the motion is observed. Other factors being equal, for example, the ground motion measured on alluvium (p.56) is generally greater than on hard rock. The medium in which the detonation occurs and the geology of the transmission path to the observation point also have some influence on the ground motion.

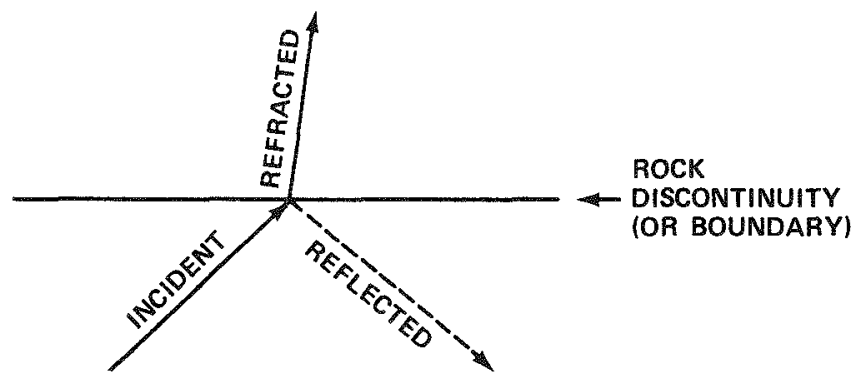

Fig. 5.2 Reflection and refraction of a seismic wave at a discontinuity.

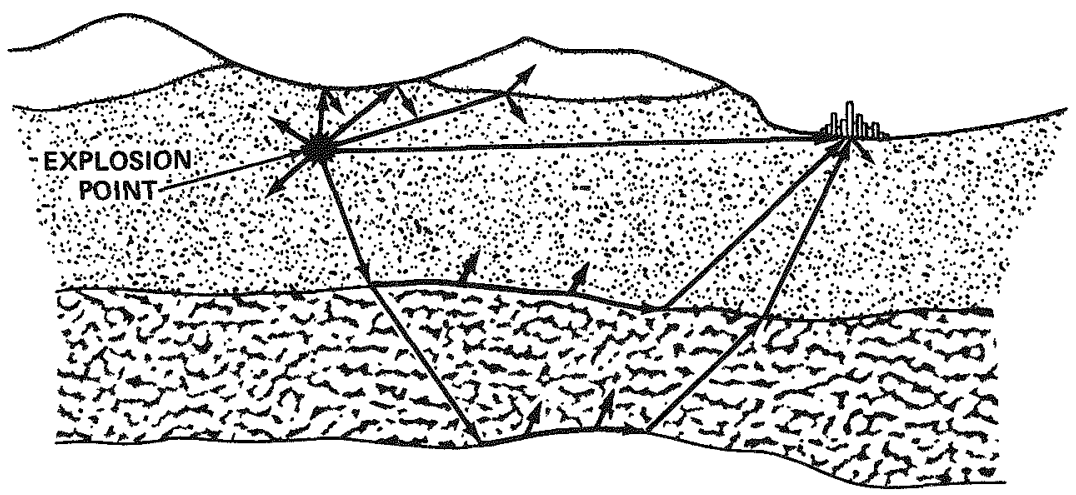

Fig. 5.3 Possible transmission paths of seismic waves from an underground explosion.

A combination of transmission path and local conditions can result in much more seismic energy being received than would be expected at the given distance from the explosion. Efforts are made to identify locations which may exhibit anomalous motion when a nuclear device is to be detonated underground. 


\section{Variations in Structural Response}

There are complexities associated with the response of a structure to ground motion just as with the motion itself. The capacity of apparently similar buildings to withstand ground motion is by no means always the same. This may be true even though the structures were designed under the same building code and constructed by the same contractor. There are unavoidable basic and inherent variations in materials, design, workmanship, etc., which lead to differences in the resistance of the structures to ground motion.

In addition to these structural variations, there are differences arising from stresses (p.47) in the structure or in the soil upon which the structure rests. Such stresses can arise from expansion or contraction (or both) in the structure and from shrinking or swelling of the soil due to changes in its moisture content. The stresses may reach the point where damage may arise from natural processes (e.g., differential settlement) without any seismic motion. On the other hand, even if the stresses are not enough for natural processes to cause any observable damage, it is possible that they may be so close to this level that otherwise minor ground motion could induce some damage.

One of the most important aspects of structural response to ground motion concerns the relationship between the characteristic (natural) vibrations of the structure and the actual vibrations of the ground due to seismic waves. This matter will be treated in some detail in a subsequent section (p.94).

\section{Measurement of Ground Motion}

Instruments called "seismometers" are used to detect ground motion and the associated response of structures. Three seismometers may be employed to observe motion in three directions at right angles to one another. These instruments are located both on the ground surface and on various structures at distances ranging from two or three miles to some 200 miles or more from the explosion point. The signals from the seismometers are amplified and recorded, frequently on magnetic tape. The components of a detecting and recording system, known as the L-7 seismograph system, which is used in connection with nuclear detonation studies, is shown in 
Fig. 5.4. Most of the seismograph stations have been operated by the U. S. Coast and Geodetic Survey (now the National Ocean Survey, National Oceanic and Atmospheric Administration), Special Projects Party, Las Vegas, Nevada.

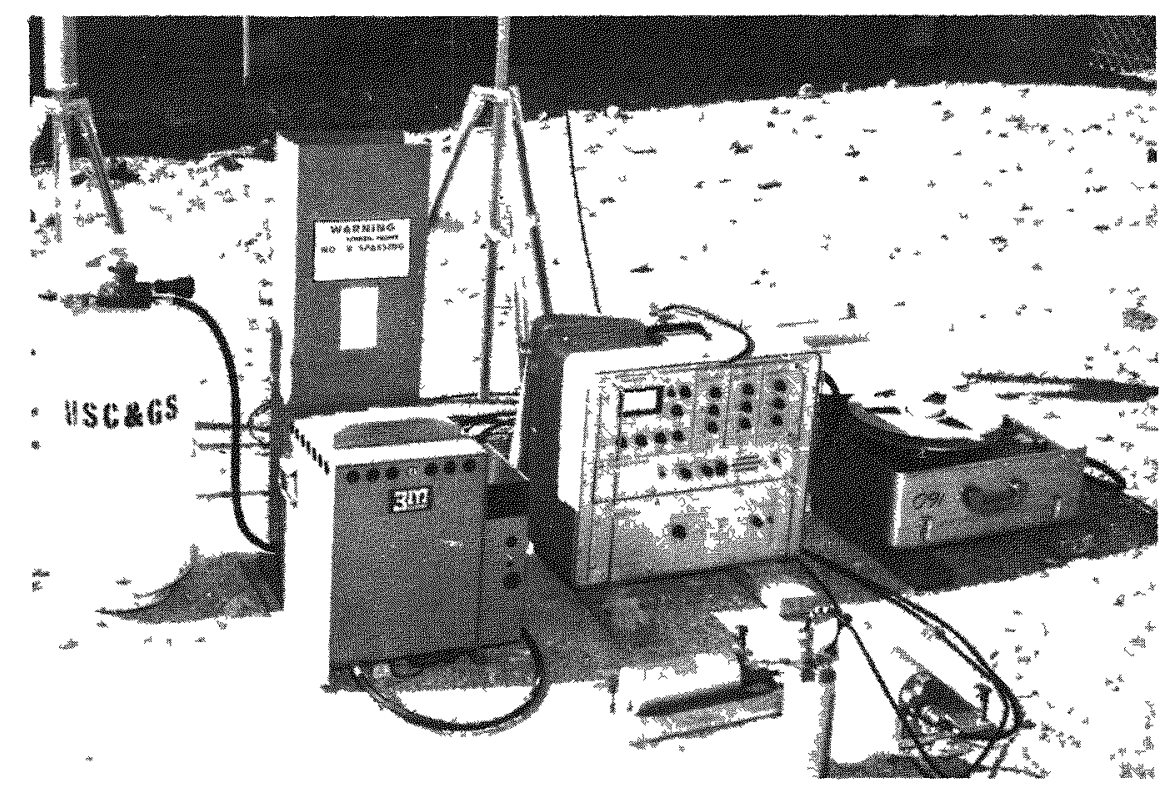

Fig. 5.4 Photograph of a L-7 seismograph system; the three seismometers are the cylindrical objects in the right foreground. (Bottled gas is used to provide electric power for operating the system.)

The number of seismic instruments used to record ground motion and their placement depends on the nature of the detonation and what data are considered to be significant for that particular event. For the Faultless calibration event, in Central Nevada in January 1968 (p. 2), about 130 seismic stations were in operation. The locations of the stations at distances of some 50 miles and more from the explosion point, identified by a star, are indicated in Fig. 5.5 .

Seismometers may respond to either acceleration, velocity, or displacement of the ground (or of a structure) over a period of time. Velocity seismometers are often used because the records (seismograms) can be readily converted into acceleration or displacement by means of a computer. An example of a seismogram showing the variation with time of the displacement of the ground at a distance 
from an underground nuclear detonation is given in Fig. 5.6. It is apparent that the ground motion is very complex and consists of many different amplitudes* at various periods.

\section{Measurement of Structural Response}

For structural response studies, especially of high-rise structures, seismometers may be placed at the top of the building, at two or more intermediate stories, in the basement, and on the ground at a distance from the building. The simultaneous recordings of motion of the structure obtained in this manner provide data on the response of the building and on the interaction of the structure with the soil upon which it stands. An example of the displacement seismograms recorded at various levels of a high-rise building following an underground nuclear detonation is given in Fig. 5.7.

Other types of structures are often instrumented to supply information on the effects of an underground nuclear detonation. In Nevada, for example, there are seismic instruments in and around Hoover Dam. The prime objective of these instruments is to monitor the behavior of the dam during earthquakes, but they also record the response (if any) from nuclear explosions.

For the Rulison event in Colorado (p.68), seismic instruments were located in many inhabited areas near the explosion site, and also on the Rifle Gap, Harvey Gap, and Vega Dams, all within 21 miles of the detonation. The dams were instrumented on the crest, on the downstream face, and on material downstream from the dam similar to that of the dam's foundation.

For the same event, more than forty instruments were installed in the U.S. Bureau of Mines Oil Shale Demonstration Mine about 10 miles away. Roof vibrations and deflections and pillar strain resulting from ground motion caused by the Rulison detonation were recorded.

\section{Other Observations}

In addition to measuring the complete time-history of the motion of the ground and of various structures affected by an

*In this book, the amplitude is the maximum displacement of the ground (or of a structure) from its undisturbed position in any single vibration of a series; the period is the time for a single cycle of vibration to occur (see Fig. 5.9). 


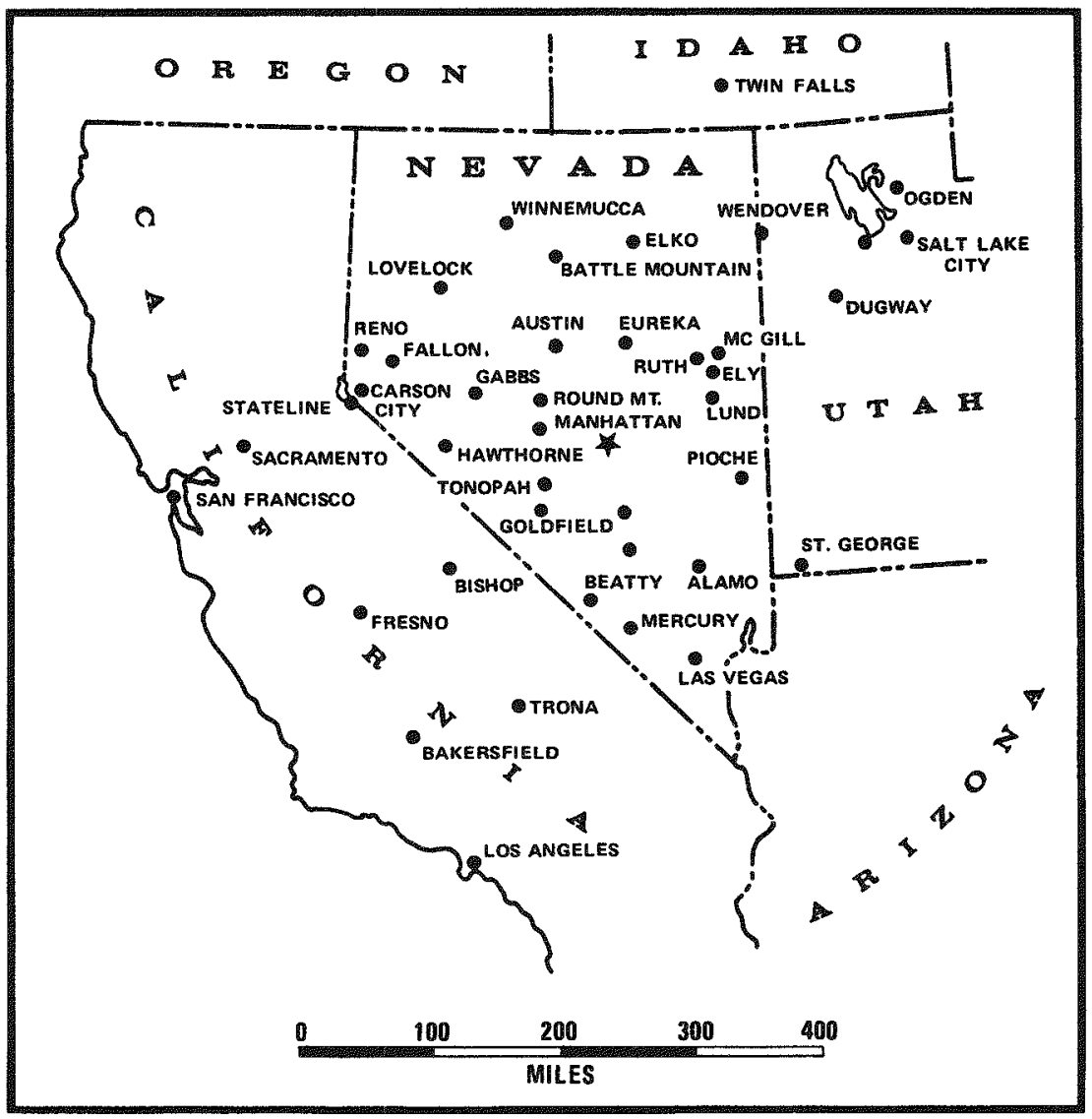

Fig. 5.5 Seismograph stations at 50 miles or more from the Faultless explosion point (indicated by the star) in Hot Creek Valley.

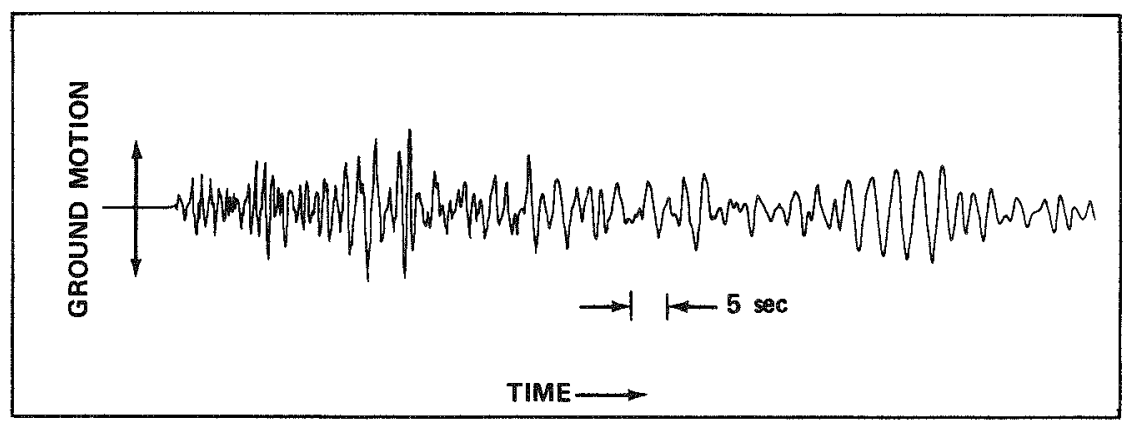

Fig. 5.6 Record of ground motion (seismogram) at some distance from an underground nuclear detonation. 


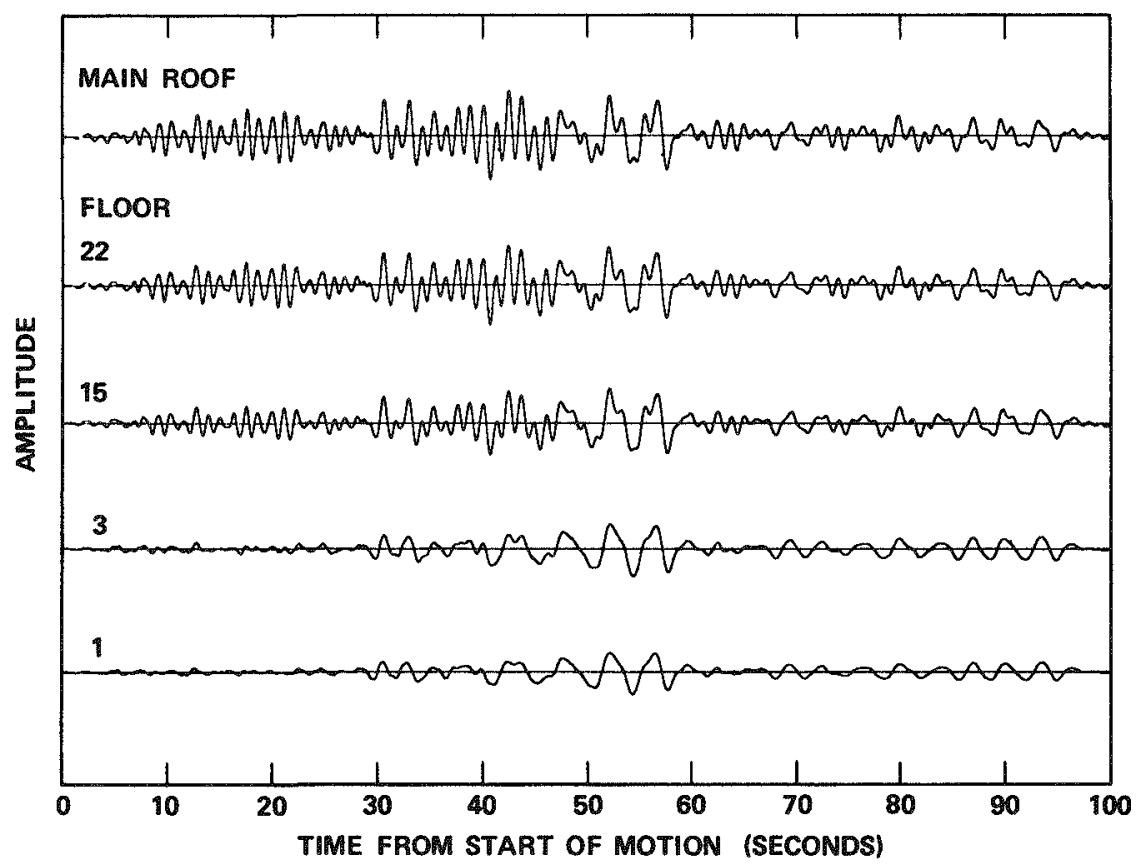

Fig. 5.7 Vibrations at different levels of a high-rise building as a result of ground motion from a nuclear detonation.

underground nuclear detonation, other information is collected. This includes observations on whether or not any damage occurred, measurement of changes (if any) in cracks in certain monitored buildings, and reports on human perception of motion.

The monitored buildings are currently some twenty structures on or near the Nevada Test Site and in Las Vegas in which existing cracks are studied regularly. Changes are noted from any and all causes, including variations in temperature and humidity, soil conditions, wind, and ground motion. The effects of an underground nuclear detonation on these buildings can thus be distinguished from the consequences of natural disturbances.

\section{NATURAL VIBRATIONS OF STRUCTURES}

\section{Causes and Characteristics of Vibrations}

It is natural for buildings and structures in general (e.g., bridges), to vibrate or oscillate to some extent. Apart from earthquakes and 
underground detonations, these vibrations can result from high winds, from sonic booms, and even from traffic on a nearby street. Consequently, structures are designed to withstand vibrations of reasonable magnitude.

Some simplified (or ideal) examples of the natural vibrations of buildings of different types are depicted in Figs. 5.8A, B, and $\mathrm{C}$.

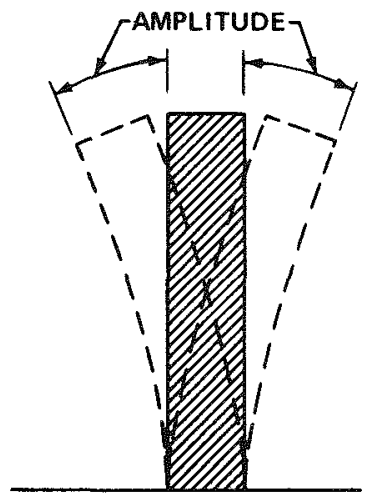

A

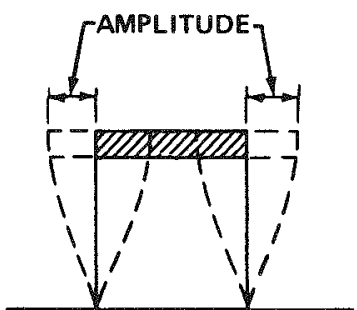

B

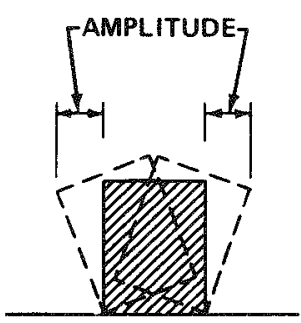

c

Fig. 5.8 Natural vibrations of idealized structures: (A) high-rise building, (B) one-story flexible frame building, (C) very rigid structure.

Thus, Fig. 5.8A might represent a high-rise structure; Fig. 5.8B might represent a flexible one-story frame building, and Fig. $5.8 \mathrm{C}$ the rocking of a very rigid structure. In each case, the undisturbed structure is shown by the solid lines, whereas the broken lines indicate - in an exaggerated manner - the extremes of the vibrational motion.

The movement from the undisturbed position of a structure to the farthest point of its motion, in a single vibration of a series, at any particular height is the amplitude of the displacement at that height. The displacement amplitude generally increases with the height above the ground; thus, motion may be felt at the top of a structure when it is not (or hardly) detectable at ground level. The time taken for the structure to move from one extreme position to the other and back again is the period of the vibration.

Every structure and indeed every element (or component) of a structure has many natural periods of vibration. For the majority of common structures, the most important of these periods is usually 
the longest one, which is associated with the simplest (fundamental) vibrational mode. This is generally a second or so for a tall building (10 to 20 stories) and a fraction of a second for a short one. Unless the structure experiences some damage, either nonstructural or structural, the natural periods of vibration do not change very much, regardless of the source of the disturbance. Minor nonstructural effects, such as partial detachment of architectural panels in high-rise buildings, have been known to produce changes on the order of 20 percent in the vibration periods. Nevertheless, for the present purpose, the vibration periods may be taken to be characteristic properties of each structure (or element).

\section{Damping of Vibrations}

If vibrations have been started in a structure, by ground motion or by any other temporary disturbance, the side-to-side movement will generally continue for some time, although with steadily decreasing amplitude, after the disturbance has died out. The variation in amplitude of a single vibration with time may be represented graphically as in Fig. 5.9; the amplitude is seen to decrease steadily until the structure comes to rest. The period of the vibration, however, changes very little, if at all.

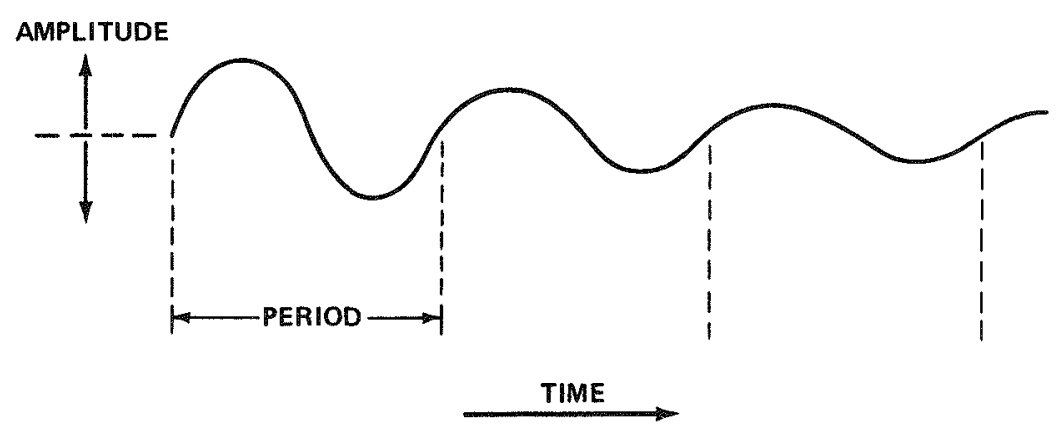

Fig. 5.9 Vibration amplitude decreases with time but the period remains essentially constant.

The property of the structure that determines how quickly the vibrations die out is called "damping." The damping represents a kind of internal friction which dissipates (or absorbs) the vibrational energy. An increase in the damping of a structure results in a more 
rapid dying out of the vibrational motion produced by a given disturbance. A structure is said to be "critically" damped when the damping is just enough to prevent it from continuing to oscillate after a disturbance has ceased, or if the structure is displaced to one side it will return immediately to the neutral (equilibrium) position without oscillating.

The degree of damping of a given structure is usually expressed as its "damping ratio," defined as the fraction (or percentage) that the actual damping is of the critical damping. Thus, if a building has a damping ratio of 0.05 (i.e., 5 percent), its damping is 5 percent of the critical damping. Observations of the damping present in typical buildings indicate that they have damping ratios of roughly 0.05 , although they might be as low as 0.01 or as high as 0.1 in certain structures. These ratios are used in calculating the expected responses of buildings to the ground motion caused by underground nuclear detonations. The effect of the damping ratio on the structural response is referred to on page 95 .

\section{Modes of Vibration}

The idealized vibrating motions (or vibrational modes) shown in Figs. 5.8A, B, and $\mathrm{C}$ are for very simple structures. Most actual structures, such as buildings and bridges, are much more complex. For example, in a typical high-rise building there are several natural modes of vibration, as will be seen shortly, but the most important is generally the fundamental mode.

There are also other complications arising from the interaction of nonstructural elements, such as interior walls, panels, plaster, etc., with the structural frame. Nevertheless, much can be learned by studying simple (ideal) systems and then applying the corrections that appear to be desirable for an actual structure.

As an illustration of the different modes of natural vibration of a structure, a high-rise building will be considered. The first three modes of vibration, based on actual measurements made on a 15-story (plus basement) office building in San Francisco, are indicated in Fig. 5.10. As before, the amplitudes of the vibrations are exaggerated in comparison with the height of the structure. At the roof, the displacement amplitude might be about 1 inch in a building 200 feet tall.

The building can vibrate in any or all of these (and other) modes at the same time. Each mode of vibration has its own period; for the 


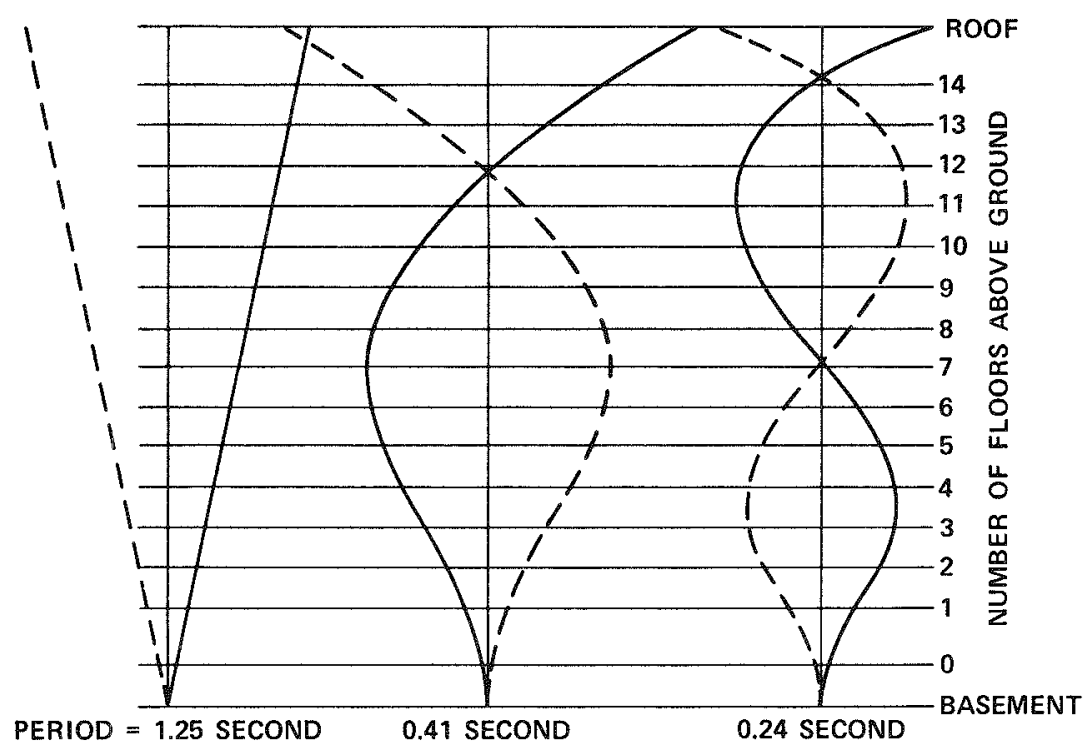

Fig. 5.10 Three modes of vibration of a tall building; the simplest, at the left, is the fundamental mode. (The vibration amplitudes are very much exaggerated in comparison with the height.)

structure referred to above, the periods of the first three modes are 1.25 (fundamental), 0.41 , and 0.24 seconds, respectively. Different types of ground motion can induce different combinations of vibrations in the various possible modes. Thus, a vibration record of a high-rise building undergoing several modes of vibration simultaneously as a result of ground motion has a complex wave form (see Fig. 5.7) which depends on the characteristics of the ground motion. However, an analysis of the record shows that it is mainly a combination of the natural modes of vibration of the structure.

\section{The Importance of Resonance}

For the matter of present interest, namely, the response of a structure to ground motion, the main point is that the response is amplified when the vibration period of the ground motion is approximately the same as one of the natural periods of the structure. The amplification effect, which is called "resonance," is illustrated in Fig. 5.11; this shows how the ratio of the maximum amplitude of the structure to that of the ground motion varies with 


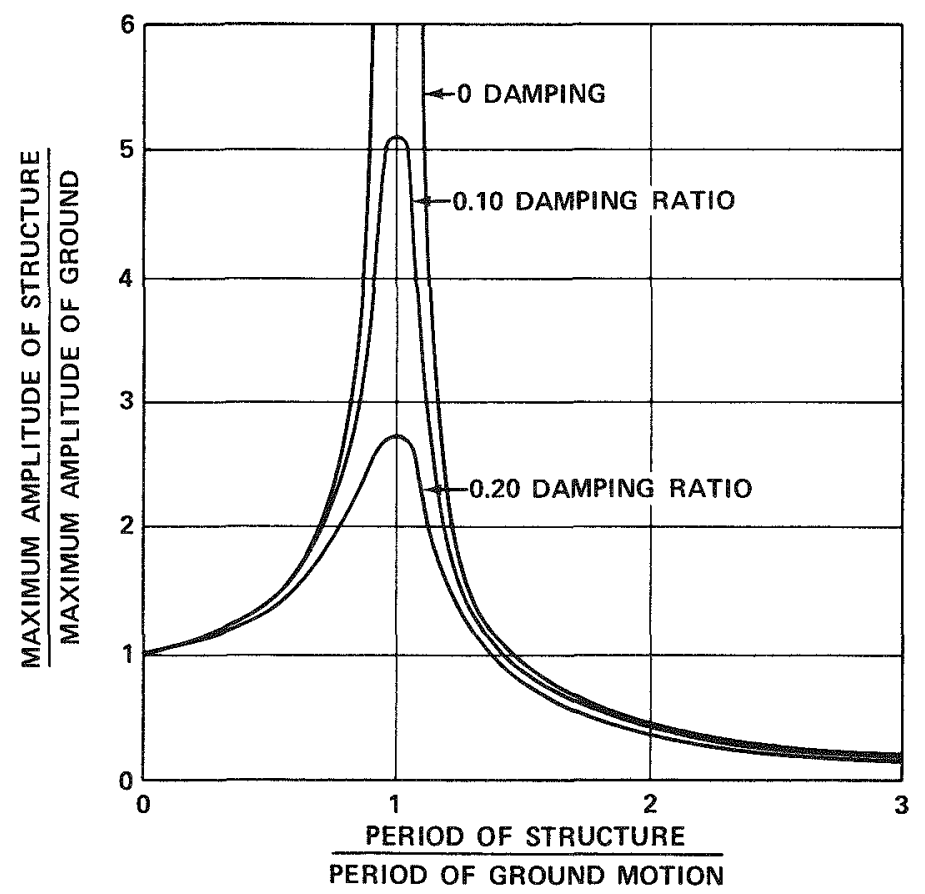

Fig. 5.11 Amplification of motion of a structure due to resonance.

the ratio of the period of the structure to that of the ground motion. The curves are for three different values of the damping. Such results as those in Fig. 5.11 are applicable only to idealized structures responding to ground motion of long duration and of only one frequency, but they are indicative of the expected trends in actual situations.

It is evident from Fig. 5.11 that the response of the structure is increased when its vibration period is roughly equal to that of the ground motion (i.e., when the ratio of the periods is in the vicinity of 1.0). When the period of the ground motion is significantly shorter or longer than that of the structure, the response is less than when the periods are about the same (i.e., at or near resonance).

The effect of damping of the structure can also be seen in Fig. 5.11. The numbers indicated are the damping ratios (or fractions of the critical damping) in each case. The more highly damped the structure, the smaller is the amplification at resonance.

It is well known that when a troop of soldiers march across a bridge they are sometimes commanded to break step. The purpose of 
this is to avoid the possibility of setting up resonance with the natural mode of vibration of the bridge. If the soldiers remain in step and the period of a stride happens to coincide with a vibration period of the bridge, the bridge could be induced to vibrate violently. As a result it might suffer damage.

The phenomenon of resonance is one of the most important factors in determining the response of structures to various disturbances. Whether resonance occurs or not depends on the vibration periods present in the ground motion and the natural period (or periods) of the structure. Thus, it is possible for one building to show a marked response to the ground motion caused by an underground nuclear detonation or an earthquake, whereas an adjacent building with a different vibration period might hardly be affected. This explains why peak ground motion by itself cannot be used to determine the response of a structure.

Ground motion caused by a distant underground nuclear detonation or by an earthquake contains vibrations of many different periods (see Fig. 5.6). But, as a general rule, at least one of the natural vibration periods of the structure is close to a period in the ground motion, so that some amplification of the ground motion by the building is to be expected due to resonance. At increasing distances from the explosion, there is a tendency for the seismic energy in the waves of shorter periods to be absorbed by the ground more readily than for waves of longer periods. In other words, at greater distances, a larger fraction of the available seismic energy appears in ground motions of longer periods. Hence, as a result of resonance, high-rise buildings, with their long (fundamental) vibration periods, often show considerable response to underground explosions some distance away whereas low-rise buildings do not.

\section{RESPONSE OF STRUCTURES TO GROUND MOTION}

\section{Studies of Structural Response}

If the precise history of the ground motion, that is, the variation of the motion with time, and the exact characteristics of a structure were known, it would be possible-at least in principle-to calculate the exact response of the structure. In practice, however, both the ground motion and the characteristics of real buildings are 
complex and are not completely understood. Because of the large number of variable factors involved, the time-history of the ground motion at some distance from an underground nuclear detonation cannot be predicted accurately at present.

In spite of the difficulties, however, considerable progress is being made in the studies undertaken to ensure safety of structures and of the people occupying them. These studies fall into two general categories.

The first is the empirical approach; this is based on actual measurements of ground motion accompanying underground nuclear detonations and the observed responses of various structures at different locations. There have been instances of nonstructural damage from underground nuclear detonations (p.111), but structural damage to inhabited structures has been virtually nonexistent. Consequently, there has been no direct information concerning the conditions under which structural damage might possibly occur. Some data have been obtained, however, from two four-story, reinforced concrete test structures located on the Nevada Test Site (Fig. 5.12) and also from destructive testing in the laboratory.

The test structures on the Nevada Test Site are used to observe the responses to underground detonations and also to determine the natural characteristics of these structures. One application is to study the effects of changes in vibration periods and damping ratios by utilizing a vibration generator bolted to a floor slab or to the roof. The speed of the vibration generator is changed in steps to produce vibrations with different periods. For each such forcing vibration period, the response of the structure is measured.

The results of a series of measurements, with the vibration generator located on the roof of the building, are given in Fig. 5.13. The resonances corresponding to three natural vibrational modes of the structure are clearly seen. There are other modes with shorter periods but they were not studied because of their lesser importance.

The second type of investigation of the response of structures to ground motion involves the use of theoretical methods. The procedures are actually semiempirical; that is to say, they are based partly on empirical (or observational) data and partly on theoretical equations. By their use it is possible to make reasonably good predictions concerning the effects of underground nuclear detonations on structures of different types. The methods for predicting ground motion from underground explosions and the responses of structures to such motion are described later. 


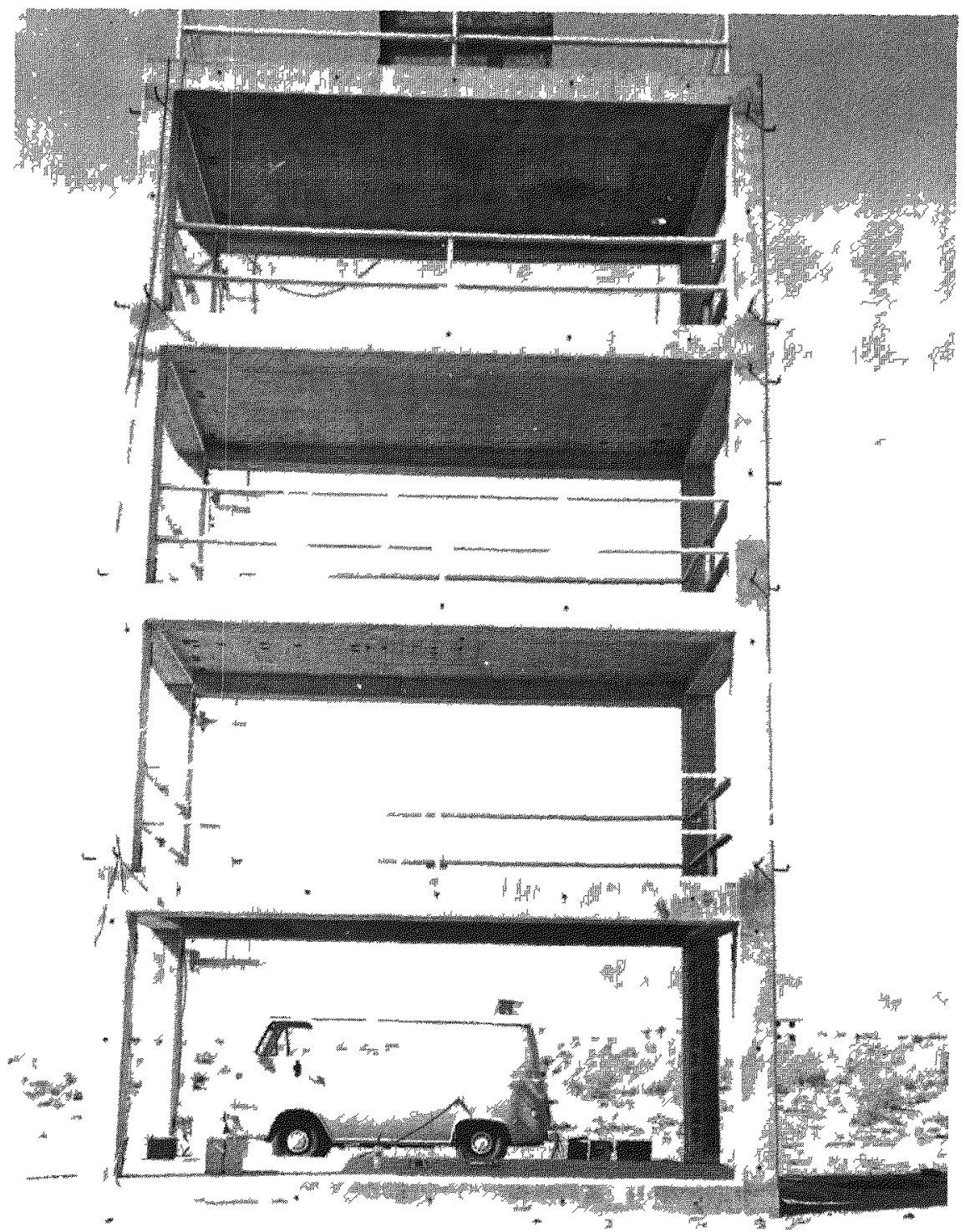

Fig 5.12 Reinforced concrete test structure on the Nevada Test Site

The Response Spectrum

One of the semiempirical methods for studying the response of structures to ground motion makes use of the "response spectrum " A linear oscillator with a single mode of vibration, which may be 


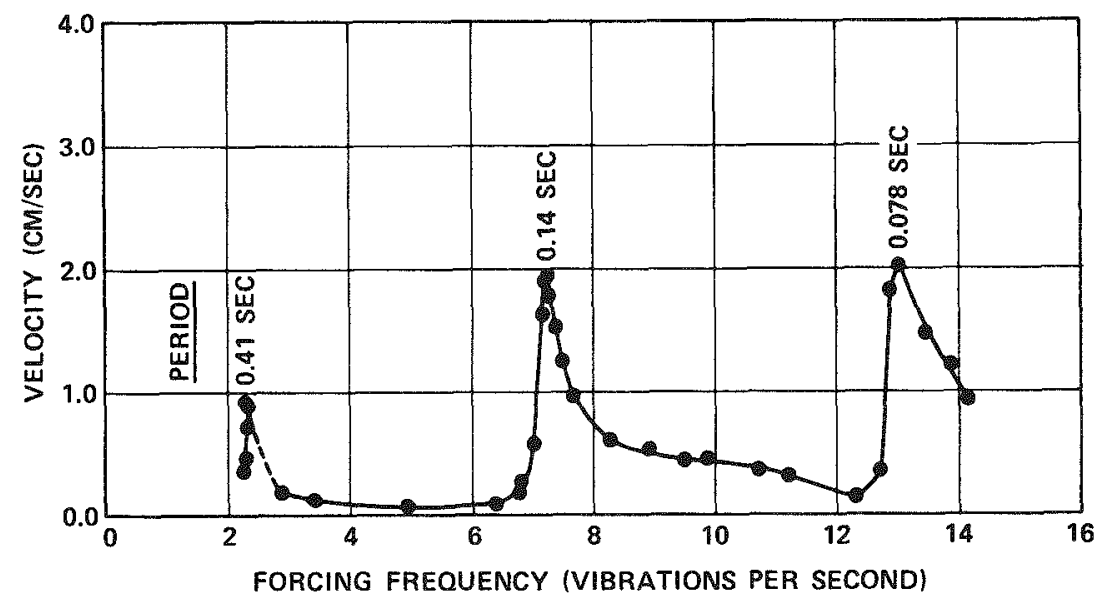

Fig. 5.13 Response of test structure to forced vibrations of the roof.

thought of as a simple idealized structure, is considered. It is assumed to be subjected to the entire time history of the ground motion as recorded by a seismic instrument at a given location. By means of mathematical equations derived from the familiar laws of motion, the maximum (or peak) response of the idealized structure can be calculated. For an elastic oscillator, that is, one which returns to its original position after the disturbance, the peak response to a given ground motion depends on only two quantities: the natural vibration period of the oscillator and the damping ratio, as defined on page 93.

A damping ratio, say 0.05 , is selected and the peak structural response is calculated for one specified vibration period, using the entire record of the ground motion at a particular location. The procedure is repeated for a range (or spectrum) of some fifty values of the structural vibration periods. The results are plotted on a special logarithmic graph paper, as shown in Fig. 5.14, to depict the response spectrum for the assumed damping ratio for the given ground motion. Because the peak velocity, displacement, and acceleration of a simple (harmonic) oscillator are related mathematically, a single curve on this graph paper gives the variation of these three quantities with the vibration period of the oscillator. The horizontal lines in Fig. 5.14 represent peak velocities (left-hand scale), those sloping downward from left to right are peak displacements, and those sloping upward from left to right are peak accelerations (right-hand scales). Vibration periods are given at the bottom of the figure. 


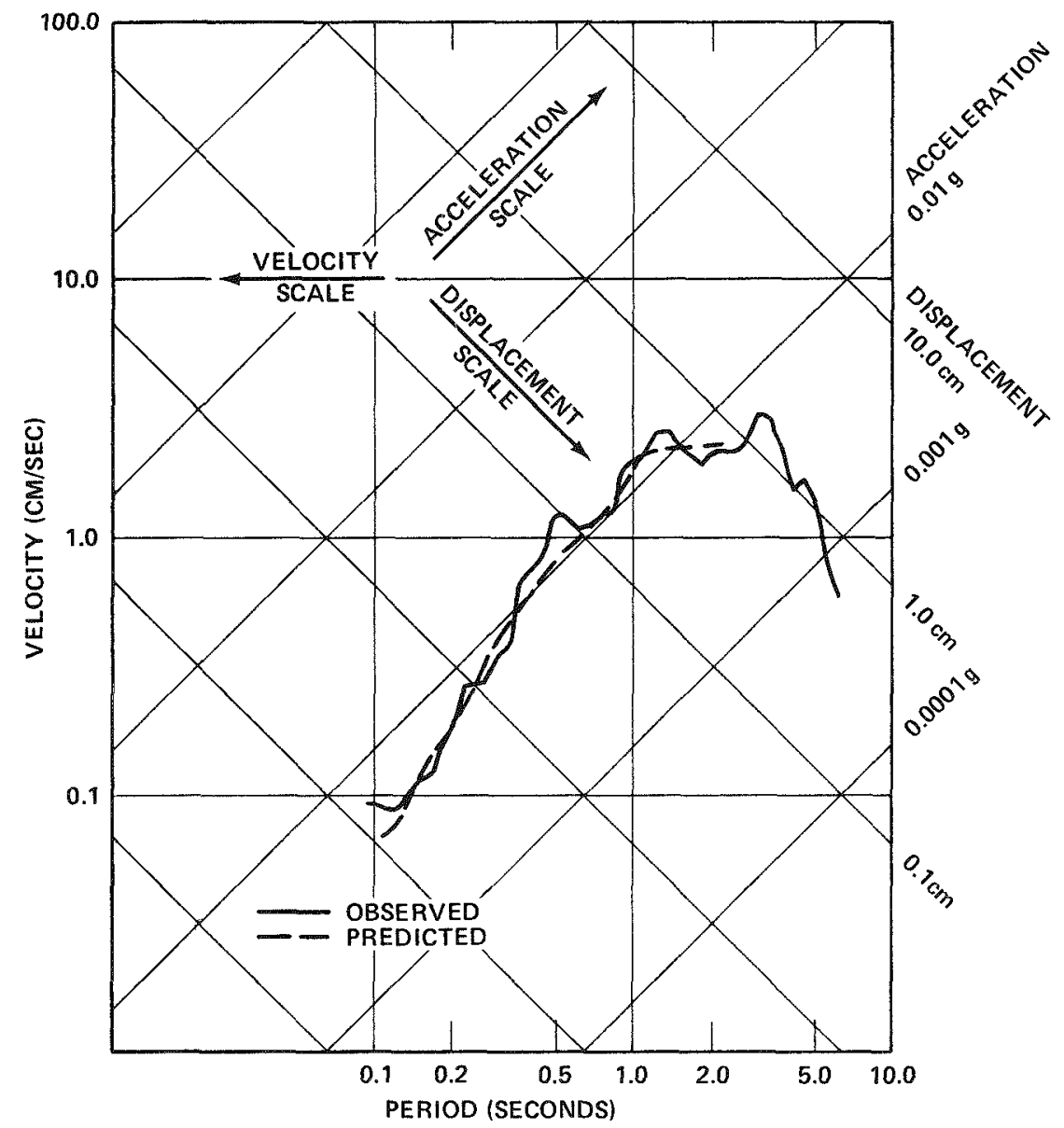

Fig. 5.14 Response spectrum at Las Vegas, Nevada, from a 1-megaton explosion at a distance of 110 miles.

The response spectrum (full line) in Fig. 5.14 was calculated for a damping ratio of 0.05 from the observed ground motion at Las Vegas (110 miles away) at the time of a detonation with a yield of about 1 megaton TNT equivalent at the Nevada Test Site. The square of the peak velocity for a specific vibration period provides an indication of the amount of energy of motion of the structure at that period. It is evident from Fig. 5.14 that, in this instance, a larger proportion of the seismic energy went into structures with longer periods $(0.5$ to 2.5 seconds) in Las Vegas than into structures with shorter periods (less than 0.5 second). 
As will be seen later, a procedure has been devised for predicting the response spectrum at a specified distance from an underground detonation of given yield. This spectrum is then used to estimate the expected response of (and possible damage to) different types of structures. By comparing the predicted response spectrum, such as the one indicated by the broken line in Fig. 5.14, with the observed spectrum, the reliability of the prediction methods can be tested. The general agreement between the two curves shows that, in this particular instance, the responses of idealized structures could have been predicted reasonably well for vibration periods between about 0.1 and 2.5 seconds.

\section{The Time-History Response Method}

In the response-spectrum method of studying the behavior of structures, as just described, an ideal oscillator with a single mode of vibration is considered and a correction is applied to give the peak response of a real structure. Although the results are approximate, the spectrum indicates the expected behavior of many structures with a range of vibration periods. A more accurate treatment, called the "time-history response" method, considers each structure individually. It should be noted that the qualification "time-history" refers to the response of the structure, since the procedure gives this response over the whole time-history of the ground motion. The response spectrum method makes use of the same time-history of the ground motion, but it yields only the peak response and not its complete time-history.

In the time-history response method, a mathematical model is made of a particular building with due allowance for various masses at different floor levels, varying stiffnesses and damping ratios, and other structural factors. The time-history record of the observed ground motion is then used with the model to compute the actual response. This is a very complex operation, possible only with high-speed computers of large capacity.

Because of the labor and complexity involved in the time-history response procedure, the method has been used only occasionally, for example, in studying the behavior of a special structure, such as a particular high-rise building. Another important application is to learn more about the behavior of real buildings with their many structural and nonstructural elements and materials. Such informa- 
tion is required for making reliable predictions of the responses of various types of buildings to underground nuclear detonations.

Among the facts that have been brought to light by studies of the kind described above is that the characteristics of some high-rise buildings may be markedly different from those intended by their designers. It has also been shown that certain methods of design that are still in use can lead to structures that have a more limited capacity to withstand horizontal (sideways) forces than intended. Results of time-history analyses have been found useful in identifying buildings that might be adversely affected by underground nuclear explosions or by earthquakes.

\section{PREDICTIONS OF GROUND MOTION AND RESPONSE SPECTRA}

\section{Introduction}

The ultimate purpose of the measurements of ground motion resulting from an underground nuclear detonation and of the methods used to calculate the accompanying response of structures is to be able to predict the effects of such a detonation in advance. Of course, if the ground motion at a given location could be predicted and if enough details were known about the buildings, it would be theoretically possible to foretell, with a good degree of accuracy, the responses of structures of various types.

Because of the large number of variable factors involved, it does not seem probable at present that the ground motion at a given location can be predicted exactly. Neither is all the required information about the building always available. Although progress is being made in both respects, the current approach is to identify and account for the variables thought to be the most significant. An estimate is then made of the uncertainty associated with the neglected variables and this is taken into consideration in the predictions of ground motion and the structural response to this motion. The probability of unexpected damage to structures from an underground nuclear detonation has always been small. But it has become even smaller over the years as more knowledge has been gained concerning ground motion and the responses of different structures to such motion. 


\section{Ground Motion Prediction}

The first step in predicting the effects of underground nuclear detonations on structures is to establish the maximum distance from the explosion point at which noticeable damage might be expected. In this respect, the peak ground acceleration has been found to be a useful general criterion. For example, there is usually no concern over damage to low-rise buildings provided the peak acceleration of the ground is less than one-hundredth of the normal gravitational acceleration (i.e., $0.01 \mathrm{~g}$ ). For high-rise structures, such as tall buildings and smokestacks, the limit is lower $(0.001 \mathrm{~g})$.

Numerous observations of ground acceleration (and related quantities) have been made with seismic instruments at various distances from underground detonations over a wide range of energy yields at the Nevada Test Site. Environmental Research Corporation, the ground motion contractor employed by the U.S. Atomic Energy Commission, has used statistical methods to analyze these data and has developed equations which relate the peak ground acceleration to the energy yield of the explosion and the distance. From these equations the peak accelerations at various locations can be predicted for detonations conducted at the Nevada Test Site.

Examples of such predictions are given by the full lines in Fig. 5.15 for an explosion with an energy yield of 1 megaton. The left side of the figure refers to peak accelerations on hard rock and the right side to alluvium. The broken lines, parallel to the full lines, indicate the range of uncertainty in the predictions. The small circles show the peak accelerations measured at various distances from the Boxcar detonation, for which the yield was close to 1 megaton. It is seen that in most instances the observed accelerations fall within the predicted range.

The predictions in Fig. 5.15 refer to typical contained explosions at the scaled depths of burial commonly employed at the Nevada Test Site (see Chapter 3). Because of the large amount of information accumulated over the course of more than three hundred underground detonations at this Site, reasonably good predictions are possible, as the figure shows. When a nuclear device is to be detonated in a new area, the results from Nevada are used as the basis for preliminary calculations. Modifications are then made, particularly for differences in the geology of the explosion medium and for the depth of burial, as may be required. 

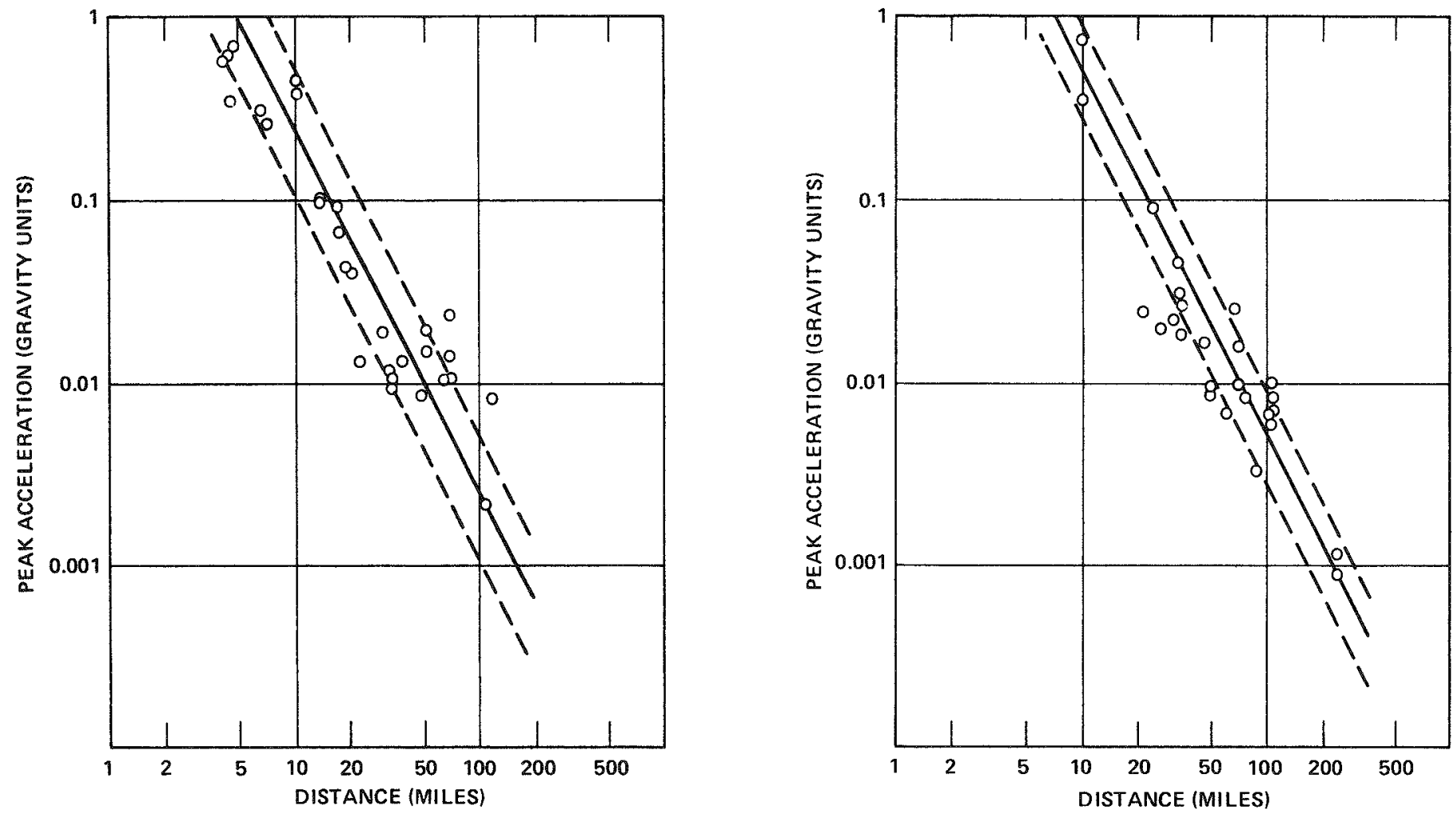

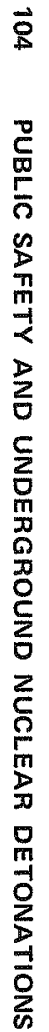

Fig. 5.15 Predicted peak accelerations on hard rock (left) and alluvium (right) from a 1-megaton explosion. (The broken lines represent the range of uncertainty.) The small circles are measured values obtained in the Boxcar test. 
In Plowshare experiments for stimulating the flow of natural gas, such as the Gasbuggy and Rulison events, the scaled depth of burial of the nuclear explosive may be much greater than is usual at the Nevada Test Site. In these circumstances, there is a substantial increase in the peak acceleration (and a decrease in displacement), especially at distances close to the explosion point. Theoretical studies by the ground motion contractor have led to the introduction of correction factors to allow for differences in the scaled depth of burial. Accurate predictions of ground motion were made for the Rulison experiment by applying these corrections to the Gasbuggy data, with scaling for yield and distance based on experience at the Nevada Test Site.

\section{Response Spectra Predictions}

Statistical analysis has also been applied by Environmental Research Corporation to the many response spectra, obtained in the manner described earlier, from seismic measurements made for Nevada Test Site events. As a result, the response spectrum at a given distance from an underground explosion of specified energy yield can now be predicted with reasonable accuracy over a range of vibration periods. A spectrum predicted in this manner, for an assumed damping ratio of 0.05 , is indicated by the broken curve in Fig. 5.14.

At the present time, there is considerable confidence in the ability of the ground motion contractor to predict response spectra for detonations at the Nevada Test Site. By allowing for the greater scaled depth of burial of the nuclear explosive and for differences in geology, reasonably good response spectra were predicted for the Rulison event. It is of interest that as the scaled depth of burial is increased, a larger proportion of the seismic energy goes into ground vibrations of shorter period. As a result, there is an increased potential for damage to low-rise (short-period) buildings, whereas that for high-rise (long-period) structures is decreased.

An examination of the predicted response spectra for various energy yields and distances has revealed some important aspects of the expected responses of structures to ground motion from underground nuclear explosions. The general conclusions are represented in a qualitative and simplified form in the accompanying figures. Figure 5.16 indicates (on a logarithmic scale) how the 
response of a simple structure would depend on the vibration period of the structure at a fixed distance from detonations of increasing energy yield. As might be expected, the response at any vibration period increases with the energy yield, but the increase is greater for longer than for shorter periods. A somewhat similar effect is evident from Fig. 5.17 which refers to various distances from an explosion of

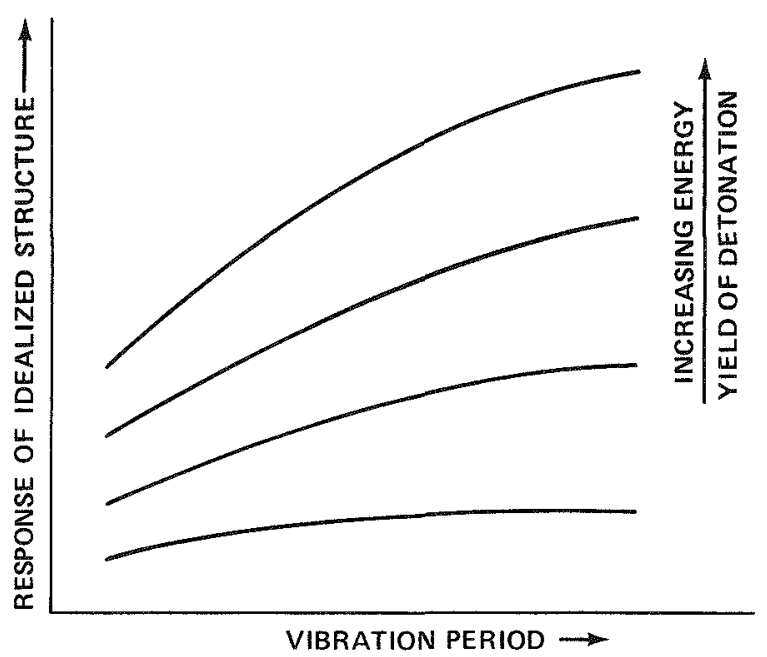

Fig. 5.16 Qualitative representation of the effect on the response spectrum of increasing energy yield at a given distance from the detonation.

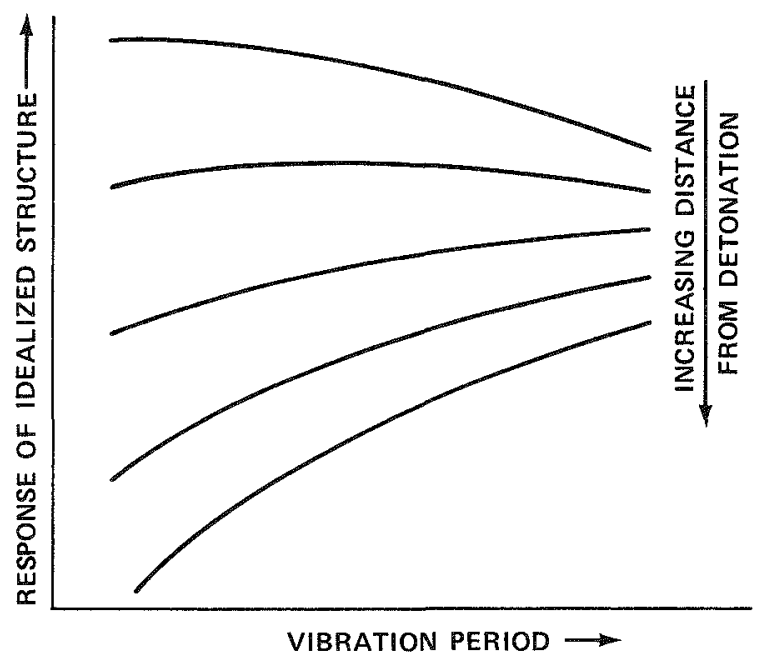

Fig. 5.17 Qualitative representation of the effect on the response spectrum of increasing distance from a detonation of given yield. 
specified energy yield. With increasing distance, the response decreases, but the decrease is relatively less for longer than for shorter vibration periods.

The foregoing conclusions, based on Nevada Test Site experience, imply that at greater distances from explosions of high energy yield there should be a tendency for a larger proportion of the available seismic energy to appear in ground motions of longer periods. As a consequence, the responses of high-rise buildings, with their longer vibration periods, are of special interest at greater distances (e.g., in Las Vegas) from detonations of high energy yield at the Nevada Test Site.

\section{DAMAGE PREDICTION}

\section{Introduction}

The peak acceleration and response spectra predictions referred to in the preceding section are used by the U.S. Atomic Energy Commission's structural response contractor, John A. Blume and Associates Research Division, San Francisco, California, to predict the effects of an underground nuclear detonation on actual structures in the vicinity of the explosion site. By comparing the capacity of structures to withstand ground motion with the predicted response spectrum, an experienced structural engineer can make an estimate of the extent of damage (if any) that might result from a particular detonation.

It will be recalled that the response spectrum applies strictly to a linear oscillator with a single mode of vibration and an assumed damping ratio. This may be identified approximately with a simple, idealized structure having the same respective vibration period and damping ratio. In real-life situations, however, buildings do not behave as ideal structures with a single vibration period and, moreover, the damping ratios vary. Consequently, allowances are included in the damage estimates for such factors as type, age, and condition of the structure, and also for other variables. In this manner, reliable predictions have been made of the effects of underground nuclear detonations on buildings and other structures.

A certain amount of nonstructural damage, such as cracking of plaster and disturbance of bricks and concrete blocks, is always possible as the result of the ground motion from an explosion. In the 
event of damage, the owners of the buildings would be compensated financially. Should the predicted damage be unacceptable, appropriate steps are taken to improve the situation, such as strengthening of weaker structures or changes in the conditions of the proposed detonation. In some cases, temporary evacuation of people from certain structures and locations may be considered advisable.

\section{Survey of Structures}

In situations where structural damage estimates are required, they are obtained by making a survey of all structures that might be subjected to significant ground motion. The area covered would extend, as a general rule, out to a distance from the explosion point where the peak ground acceleration is $0.01 \mathrm{~g}$. High-rise structures at greater distances would, however, be included because of their susceptibility to smaller accelerations (p.103).

In making the survey, the area of interest would be first divided into units, possibly cities, towns, or other regions, within which the characteristics of the ground motion caused by the nuclear detonation are not expected to vary greatly. If, however, geologic or other local circumstances, such as different areas where the buildings are either all old or all new, indicate that some variations are possible, the unit may be subdivided into zones (Fig. 5.18). Thus, a knowledge of the geologic conditions is necessary.

The types and characteristics of the structures in the area would be determined and then the structures classified into groups, according to their natural periods of vibration. Each group would include buildings whose periods fall within a narrow range. In other words, the natural periods of the buildings within each of the groups are so close that they can be treated as having essentially the same period.

Although the buildings within each group may be assumed to have the same period, they would not all respond in the same manner to a given ground motion. For example, there will be variations in age and condition that must be taken into consideration. Consequently, a further subdivision of structures into subgroups would be made within each group.

The classification of the buildings into groups, according to their vibration periods, and into subgroups, according to their characteristics, would be made for each zone, as defined above. Finally, the 


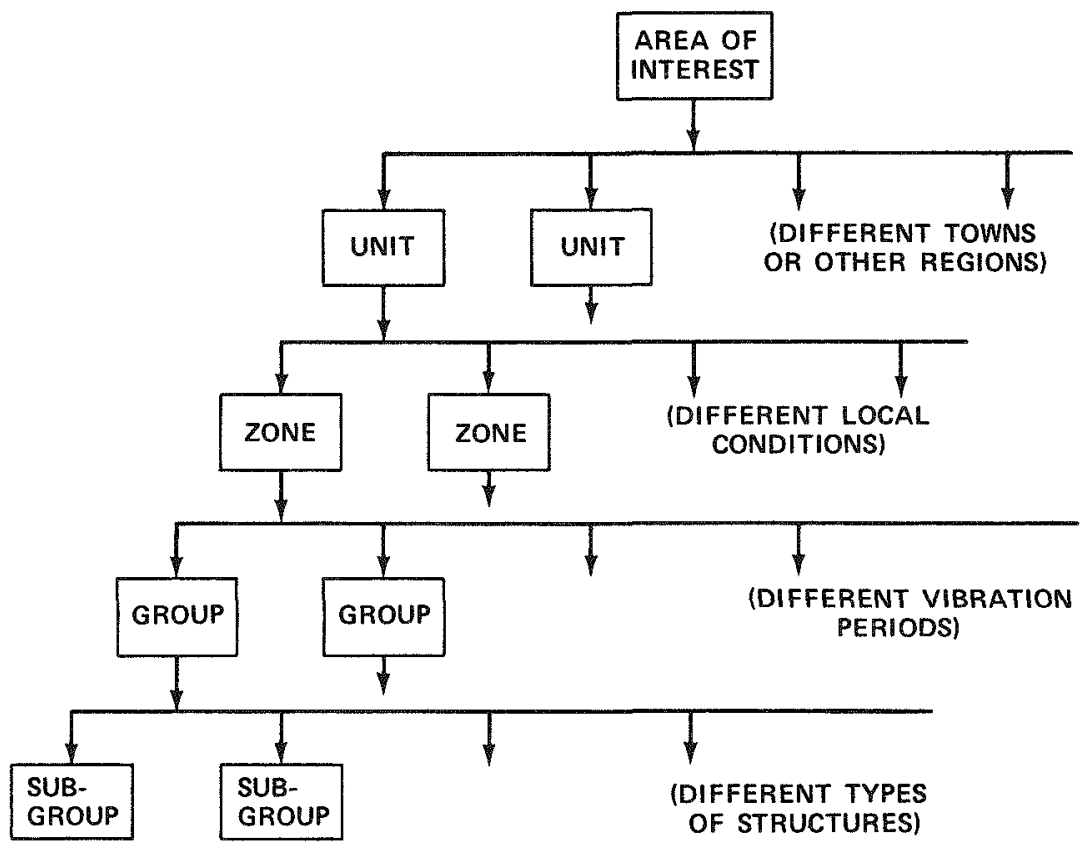

Fig. 5.18 Possible classification of structures in a survey.

dollar values of all types of structure that might be damaged would be recorded.

\section{Estimates of Probable Damage}

The procedure just described involves essentially a breakdown of all structures that might be affected by the nuclear detonation into a large number of subgroups, each consisting of structures with similar characteristics and damage susceptibility. An estimate of the probable damage to each subgroup is then made from the predicted response spectra. Summation of the damage costs for all the subgroups in the area surveyed would then give the total estimated damage. Since there are unavoidable uncertainties both in the predicted response spectra and in the capacities of similar structures to withstand ground motion, the damage estimates are expressed in terms of probabilities.

The calculation of these probabilities, which is in effect an extensive bookkeeping operation using many pieces of information, is conveniently performed by means of a computer. Furthermore, 
with a computer the effects of small variations in the input data can be readily determined. This provides an indication of the degree of confidence that may be placed in the damage estimates.

An example of the kind of results obtained in a damage estimate calculation is given in Fig. 5.19; the curve shows the damage probability versus the dollar value of the structural damage. The

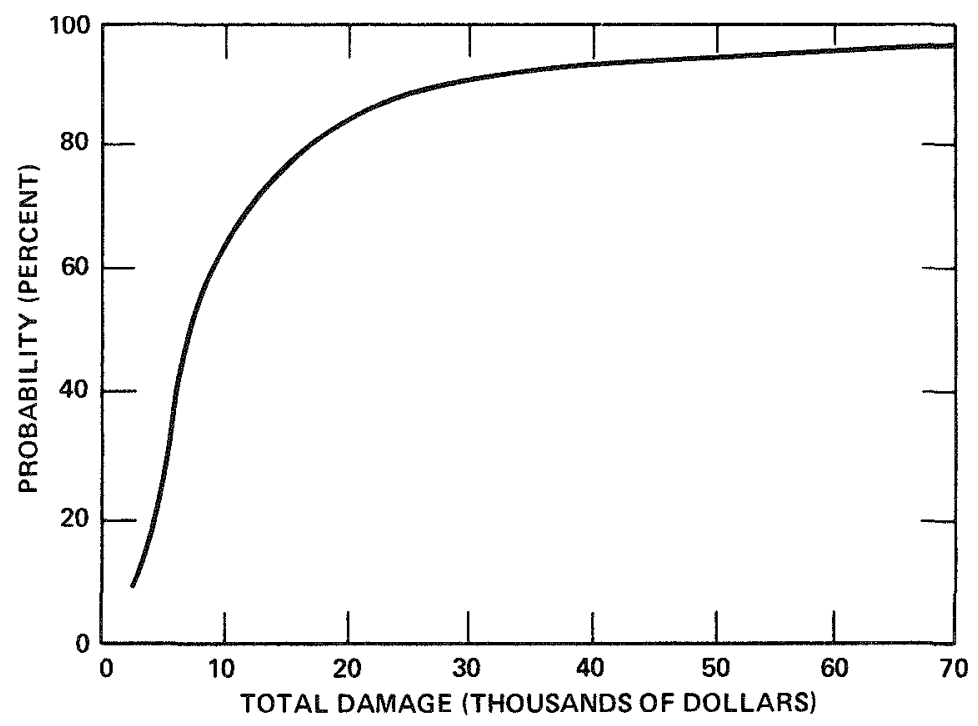

Fig. 5.19 Results of damage calculations (hypothetical).

probability on the vertical scale represents the chance (expressed as a percentage) that the actual damage will not exceed the corresponding value as read on the horizontal scale. Thus, in this case, there is a 50 percent chance that the damage cost will not exceed $\$ 9,000$ and a 90 percent chance that it will not exceed $\$ 25,000$.

If the area in which significant ground motion is to be expected includes a large city such as Las Vegas, Nevada, with buildings of high value, a special analysis may be performed for some individual structures. An analysis of this kind, made by the structural response contractor, led to the decision by the Atomic Energy Commission to strengthen the shear wall in a high-rise building in Las Vegas. This structural change not only improved the building's capacity to withstand ground motion from nuclear detonations, but it also greatly enhanced its earthquake resistance. 


\section{Experience of Damage to Structures}

The first significant experience of damage to structures as the result of an underground nuclear detonation was at the Salmon event (p.39). An unexpectedly large number of structures in Hattiesburg some 25 miles away suffered nonstructural damage, chiefly cracking of plastered walls and ceilings, of stucco, and of masonry (concreteblock and brick) walls. The type of damage observed was similar to that caused by naturally occurring phenomena, such as differential settlement, shrinkage of mortar due to drying, and expansion and contraction of materials resulting from temperature changes and humidity. Since Hattiesburg was well beyond the limit at which any significant response had been expected, this city was not included in the survey made before the detonation.

In many cases, poor foundation structures and soils and the inherent vulnerability of some masonry structures to ground motion undoubtedly contributed to the damage noted after the underground explosion. Furthermore, the Salmon event was conducted soon after passage of a hurricane and this may also have contributed to the claimed damage.

Prior to the Handley test on March 26, 1970, there had been little evidence of damage to any structure in Nevada (off the Test Site) that could be attributed to an underground explosion. The detonation of the Benham device in December 1968, with a high yield of 1.1 megatons TNT equivalent, however, had resulted in some minor damage to residential structures. This damage consisted mainly of cracking of plaster that could be readily repaired.

The detonation of the Handley device, with an energy yield somewhat greater than 1 megaton, resulted in nonstructural damage to 42 buildings in Las Vegas, Nevada. Claims were paid for damage to plaster and ornamental blocks and for cracks in masonry walls.

Three damage complaints were received after the Gasbuggy experiment in New Mexico (p. 48). Only one, from a ranch about 25 miles from the explosion point, was found to be valid. The damage apparently resulted from the aggravation of prior cracking by slight ground motion from the detonation.

Some nonstructural damage had been predicted before the Rulison event in Colorado (p. 68) and as a safety precaution people were evacuated from houses in the immediate vicinity of the explosion site. A total of 455 complaints were received of damage 
reputedly caused by the detonation; about 70 percent were found to be valid and the owners of the buildings were compensated without delay. The great majority of the complaints were for brick chimney damage (Fig. 5.20) and cracked interior plaster. There was also some cracking of exterior masonry (brick, stone, and block) and other walls and broken windows. In no case was the habitability of any structure affected.

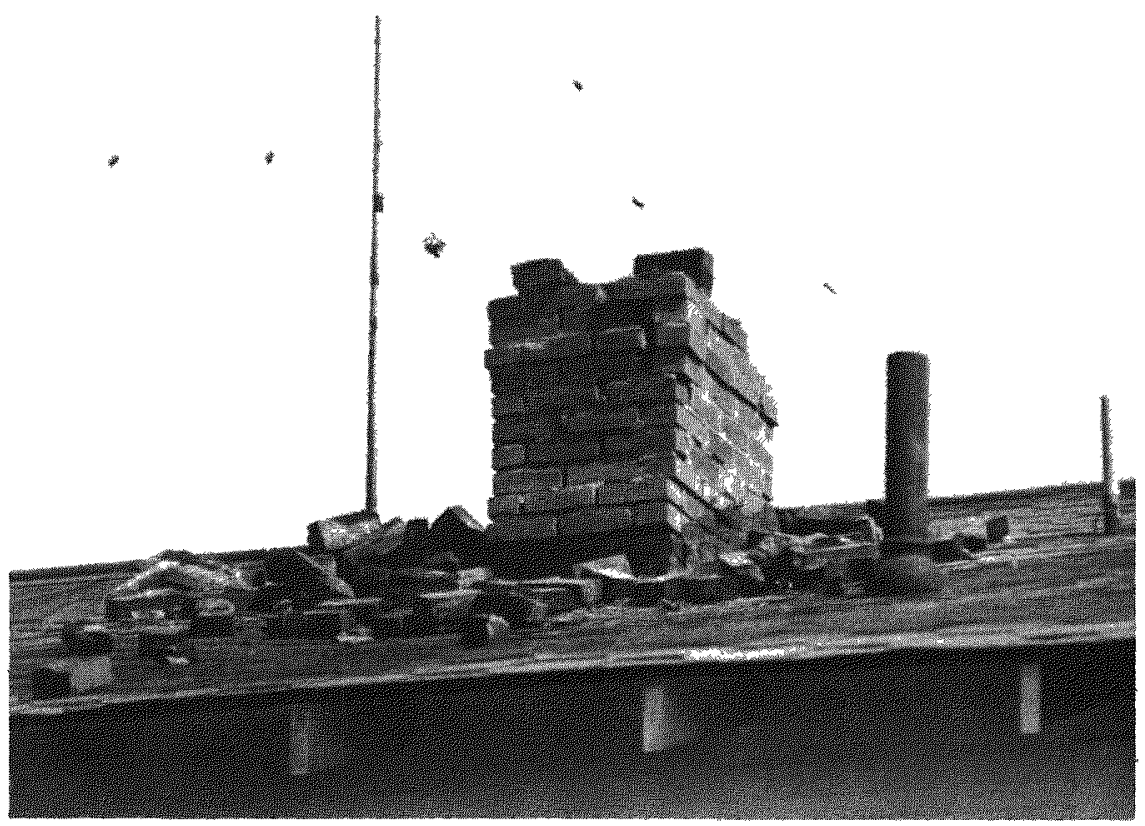

Fig. 5.20 Brick chimney damaged by the Rulison explosion.

In numerous instances where damage has been reported after an underground nuclear detonation, pre-existing conditions have been an important factor. For example, as a result of weathering and of repeated heating and cooling over the years, mortar joints of many chimneys in the Rulison area had become loose and the bricks were not bonded. Consequently, the ground motion due to the detonation caused the bricks to fall. Several of the chimneys damaged were noted in the survey made by the structural response contractor before the detonation to be a possible hazard in this respect. Newer chimneys in the same locations as the damaged ones were not affected by the explosion. 
RESPONSE OF UNDERGROUND AND RELATED STRUCTURES

\section{Introduction}

The discussion so far has referred in particular to structures, such as various kinds of buildings, above the ground. There is a possibility that other types of structures, for example, tunnels, mines, and wells, may be affected by the ground motion associated with an underground nuclear explosion. Although dams and steep slopes are not underground structures, they may be considered in the same general category.

Prediction of the effects of ground motion from a nuclear detonation on underground and related structures is based mainly on experience gained from other underground detonations and earthquakes. As a result, general criteria have been developed to indicate the extent of ground motion below which there will usually be no damage. By comparing this minimum level with the expected ground motion, as calculated for the proposed detonation, the probability of damage (if any) can be predicted.

The general criteria provide some indication of possible damage. But the actual physical conditions of tunnels, mines, and wells are variable from one structure to another of the same apparent type. Consequently, each case must be considered separately. The same is true for the effects on steep slopes; general criteria are useful but the actual prediction is based on experience gained from the observed effects of previous ground motions on these or similar slopes.

\section{Safety of Dams}

In estimating the effects of ground motion on dams, each such structure must be evaluated individually. By the use of standard engineering techniques, an idea can be obtained of the ability of a dam to withstand ground motion. In some instances, however, there may be unusual circumstances that make special attention necessary. Such was the case for the Harvey Gap Dam in Colorado in connection with the Rulison project.

This earthen dam, about 20 miles from the detonation point, had originally been lower than at present and had suffered failure. It had been repaired and its level raised by construction of a new section in 1910. The new section had apparently settled about 4 feet by early 
1969. Because of these considerations and the expected high reservoir water level at the time of the planned May detonation date, some uncertainty was felt about the ability of the Harvey Gap Dam to withstand the ground motion expected from the Rulison explosion.

For these and other reasons the detonation was postponed from May until September 1969. At this time, the level of the water in the reservoir would be lowered, by normal withdrawal for irrigation purposes, to at least 15 feet below the crest of the dam. Furthermore, by September the earth in the dam structure above the water level would have dried out to a considerable extent. Both of these factors were expected to reduce greatly the vulnerability of the dam to ground motion.

As a precautionary measure, preliminary arrangements were made to notify people in the area downstream from the dam to evacuate their homes in case examination after the detonation indicated the possibility of an uncontrolled discharge of water from the reservoir. But this precaution proved to be unnecessary. The Harvey Gap Dam, with its reduced water level, suffered no damage as a result of the Rulison detonation.

\section{Mine and Well Inspection Program}

There are two main objectives of the Mine and Well Inspection Program which is carried out by the U. S. Bureau of Mines in connection with underground nuclear detonations. The first is to ensure the safety of mines and oil and gas wells, and their operating personnel. The second is to establish a system of controls to assess and evaluate any damage to a mine or well that may be reported after a nuclear detonation. Information from the program can be used to determine whether the damage is or is not to be attributed to the effects of the explosion, specifically ground motion.

Prior to a nuclear event, a general survey would be made of the mines and oil and gas wells in the area of the explosion site. From these, a number may be selected for detailed study both before and after the underground detonation. Areas in each mine or well that are considered to be vulnerable to motion would be pinpointed for the observation of changes that may occur as a result of the nuclear explosion. In the course of the inspection weak features may be revealed and recommendations would be made that they be 


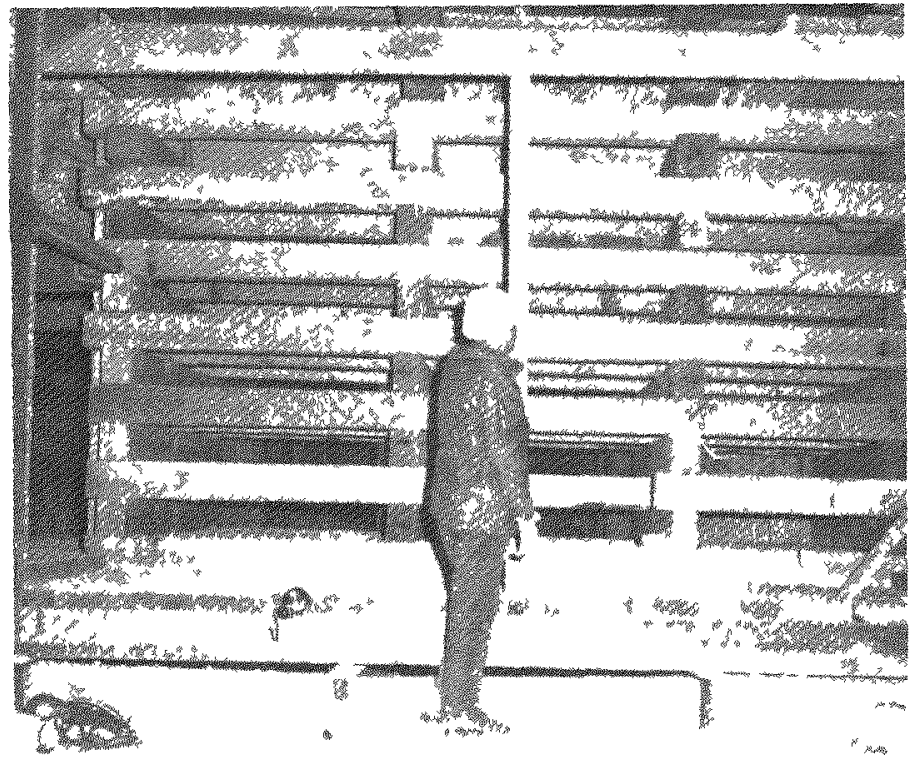

Fig. 5.21 Protective shoring installed in a mine shaft.

strengthened (F1g. 5.21). Other measures may be suggested to prevent or reduce potential damage to a mine or well that may result from earth motion of any kind.

\section{Inspection of Mines}

In underground mines, the shaft is the most important single structural feature. Men and material enter the mine through the shafts and the ore is removed through them Possibility of damage to a shaft is greater than to the mine tunnels and repair is more expensive. Consequently, special attention would be pard to the inspection of mine shafts.

In the course of the inspection made before a nuclear test, cracks in the shaft linings or in the rock walls would be noted. Weaknesses in any timber or steel structural members and other features that might be affected by the planned detonation would also be recorded. Remedial measures may then be taken where necessary and practical. Photography plays an important role in the inspection program 
because comparison of before and after photographs can provide an indication of the effects of a nuclear detonation.

Working areas of mines would also be inspected. A detailed examination of all the working areas in large mines is not practical. Consequently, those areas that are deemed critical, that is, most sensitive to ground motion, would be selected for study.

One and possibly two inspections before the detonation and at least one after it would be scheduled for each mine. A predetonation inspection would be made as close as possible to the planned explosion time and another inspection as soon as possible thereafter. In addition to these inspections immediately before and after the explosion, various measurements may be made over a period of time. Seismic instruments, for example, could record minor ground motions for several days. Furthermore, pumping records would indicate if water inflow into the mine has increased, decreased, or remained unchanged after the underground detonation. Changes, if any, in water table levels would also be noted.

Other continuing observations may be made of roof sag or of the convergence of roof and floor in mine tunnels. Long metal pins have been inserted in a special way into the roof and floor of a tunnel. An instrument called an "extensometer" is used to measure the distance between roof and floor pins to within a one-thousandth part of an inch. Any shortening of the distances between the pins that occurs after installation would indicate movement of the roof or floor or both.

\section{Inspection of Oil and Gas Wells}

Because of structural and other differences between oil and gas wells, on the one hand, and mines, on the other hand, the inspections and records would not be the same. Since inspection of the bore of a well is rarely possible, it is necessary to rely on pre-existing records. For wells of special interest, because of their known condition or location, details of casing installation and condition, well pressures, and other special features may be compiled. Furthermore, seismic instruments may be installed in locations where it appears that useful information could be obtained.

\section{Evacuation of Workers}

Workers may be evacuated from underground and surface mines and from oil and gas well area facilities in the near vicinity at the 
time of a nuclear detonation. Special re-entry teams, which have inspected the mine or well area before the explosion, would make an inspection after the explosion to assure that work can be resumed with complete safety.

\section{IMPROVEMENT OF PREDICTION TECHNIQUES}

\section{Studies of Ground Motion}

Advances have been made in recent years in connection with the prediction of possible damage to structures from ground motion caused by underground nuclear detonations. It is certain that this progress will continue as a result of several studies that are in hand to improve the understanding of all the stages, beginning with the nuclear explosion and ending with the responses of various types of structures and their components.

The characteristics of the seismic waves propagated from an underground nuclear detonation depend on the geology of the region. In practice, the geologic conditions in the area between the explosion site and a distant structure are so complex that they cannot be treated theoretically. Nevertheless, valuable clues can often be obtained from a mathematical study of a greatly simplified model of the geology. The results serve, in certain circumstances, to account for local variations from average experience. In this way, the probability of unexpected seismic effects arising from a nuclear detonation is being reduced.

A major problem in making accurate calculations of the response of a structure, even when a fairly complete mathematical model of the structure is available, is the prediction of the ground motion. It is unlikely that sufficient information will be available in the foreseeable future to permit the exact form of the ground motion, as shown by the seismogram in Fig. 5.6, to be predicted. However, "typical" ground-motion records are being developed theoretically which have the correct amplitude and vibration period content for use in the analysis of actual buildings by the time-history method (p.101). Better predictions should thus be possible of the effects of underground detonations on particular structures of interest.

It is clear from Fig. 5.15 that ground motion on alluvium is substantially greater than on hard rock; this increase is larger at some 
vibration periods than at others. The degree of amplification, in fact, depends in a complicated manner on both the type of seismic wave and on its vibration periods. A fundamental study is being made of the propagation of different wave types in an alluvial layer. The objective is to determine the effect of the layer on the observed ground motion at different vibration periods. As a result of these continuing studies, there has been a substantial improvement in the prediction of response spectra at sites located on alluvium.

\section{Studies of Structural Response}

Predicted and actual responses of various types of structures to ground motion are compared statistically by response spectrum techniques to evaluate the accuracy of present prediction methods. Thus, it is possible to determine how well the idealized models used in mathematical analysis correspond to the structure as actually built. In this way better models, as well as new theoretical approaches, can be developed and tested.

Because there has been no structural damage to high-rise buildings caused by underground nuclear detonations, information on damage thresholds is obtained from the four-story test structures at the Nevada Test Site referred to on page 97. These have been subjected to forced vibrations developed by a vibration generator as well as to the ground motion from nearby underground detonations. In addition, laboratory measurements are providing data on the behavior of many different kinds of partition walls and wall materials.

A continuing survey is in progress to monitor existing conditions in a number of buildings in order to observe changes that may occur, especially as a result of underground nuclear detonations of large energy yield. These buildings include low-rise and high-rise structures in Las Vegas as well as various structures nearer to and on the Nevada Test Site.

An active program is under way to determine the properties of foundation materials in regions exposed to ground motion from underground nuclear detonations. By means of properly placed instruments, studies of the interaction of a structure with its foundation soil are made concurrently with the forced vibration testing of the four-story test structures mentioned above. From the data obtained in this manner, the influence of the interaction of the 
soil and the structure on the response to ground motion is being evaluated.

A comparison of the effects of nuclear detonations at the Nevada Test Site on the ground motion near a church in Tonopah, Nevada, and at a motel some 200 feet away is of interest in this respect. The church stands on an outcrop of rock, whereas the motel was built on a pile of mine tailings. Analysis showed that the motion of the ground at the motel would be amplified by the mine tailings and should thus be appreciably greater than the ground motion at the church. This has proved to be the case.

In some instances, the predictions may indicate that a planned underground nuclear explosion will cause some damage to existing buildings. If these buildings are relatively small in number, then bracing or other measures can be recommended. But if an entire city or other populated area is involved this is not practical. The damage potential must then be decreased by modifying the conditions for conducting the detonation, such as a change in the energy yield or in the location and depth of the explosion point. Various methods for preventing or minimizing damage to buildings are being studied and developed.

\section{SUMMARY}

An underground nuclear detonation produces a seismic wave that causes the ground at some distance away to undergo complex motions. The effect of these ground motions on a structure depends on the characteristic vibration period (or periods) of the structure. Ground motion of the same vibration period as the structure can produce a significant response but motions with different periods have a smaller effect. High-rise buildings respond to ground vibrations with longer periods, whereas low-rise buildings are affected more by ground motions of shorter periods.

In the response spectrum method of analysis, the response of a simple oscillator, with a single vibration period, to the groundmotion record is calculated. A plot of the results over a range of periods gives the response spectrum at a particular location. By using data accumulated from many nuclear events at the Nevada Test Site, equations have been derived from which response spectra can be predicted. Response spectra have indicated that special importance 
must be attached to the effects of detonations of high energy yield on high-rise buildings at a distance from the explosion point.

In the time-history method of studying structural response, the observed ground-motion record is used to compute the response of the components of a particular building by applying the record to a mathematical model of the building. Important information concerning the characteristics of the building, which are not readily apparent, are obtained in this manner. Ground-motion prediction techniques are being developed theoretically which can be used in the time-history method to analyze the expected response of individual structures to underground explosions.

If it appears desirable, a survey is undertaken prior to a nuclear test of the structures within the area where significant ground motion is predicted. An estimate can then be made of the probable damage (if any) that may result from the planned detonation. Should this appear to be unacceptable, suitable changes are made to reduce the potential damage. In some cases, the survey may reveal existing defects in structures that can be rectified at a relatively small cost. 


\section{Chapter 6 \\ SEISMOLOGY AND \\ WATER WAVE PHENOMENA}

\section{INTRODUCTION}

\section{Aftershock and Other Effects}

It has been known for some time that, in addition to producing the ground motion described in Chapter 5, underground nuclear detonations are followed by a number of minor seismic tremors called "aftershocks." (This term is the same as that used to describe the secondary tremors that usually occur after the main shock of a large earthquake.) In many instances, the aftershocks from underground explosions have been directly related to the postdetonation phenomena of cavity collapse and chimney growth (Chapter 3 ).

In recent years, however, it has been observed that underground detonations in the higher yield range at the Nevada Test Site are often accompanied by aftershocks that originate near the explosion point but outside the region involved in chimney growth. These aftershocks are generally considered to result from small movements along pre-existing fault planes and to represent the release of natural strain (deformation) energy.

Aftershocks from nuclear events have in no way constituted a danger to people or to structures off the Test Site. Nevertheless, they have focused attention on the general area of concern related to the probability of triggering significant earthquakes as the yields of nuclear explosions are increased or as other test sites are used. Consequently, the problem has been receiving a great deal of 
attention. In particular, the U.S. Atomic Energy Commission is supporting an extensive program by various universities and Government agencies to obtain objective relevant data on seismic phenomena associated with underground nuclear detonations of high energy yield. It may be mentioned that, although many detonations, with yields ranging up to somewhat more than a million tons of TNT equivalent, have been conducted at the Nevada Test Site, no relationship has been found to the occurrence of natural earthquakes in the vicinity (p. 131).

In order to understand better the connection of underground nuclear detonations with subsequent seismic effects, events of high yield, since 1968, have been extensively instrumented for the detailed observation of aftershocks. Very few of these aftershocks have been found to originate beyond about 12 miles or so from the respective explosion points and no seismic activity associated with these nuclear detonations was detected at distances greater than 25 miles.

The energies of the largest aftershocks recorded to date for any underground nuclear detonation have never been more than a few percent of the seismic energy generated by the detonation (p. 36). In the great majority of cases the aftershock energies have been an even smaller percentage of the initial seismic energy. It was seen in Chapter 5 that most people can detect low levels of ground motion. Yet, so far as is known, no one outside the immediate test area has reported feeling an aftershock from a nuclear event.

In addition to aftershocks, underground nuclear explosions in the higher yield range can cause displacement of neighboring faults. Such displacements are discussed in the first part of the next section. This is followed by a more detailed description of the aftershock phenomena referred to above. The question of the formation of potentially destructive water waves, as a result of the seismic effects of underground nuclear detonations, is considered at the end of the chapter.

\section{Definitions of Terms}

Before proceeding, some of the terms to be used in the following sections will be defined with the aid of Fig. 6.1. The "hypocenter" (or "focus") of an earthquake is the location on a fault where the motion (or slip) responsible for the earthquake started. In Fig. 6.1, the fault shown is a hidden one, and the displacement is not observed 
at the earth's surface (p. 61). However, significant earthquakes sometimes originate at faults that are apparent at or near the surface.

The "epicenter" of the earthquake is the point on the earth's surface vertically above the hypocenter. The distance between the

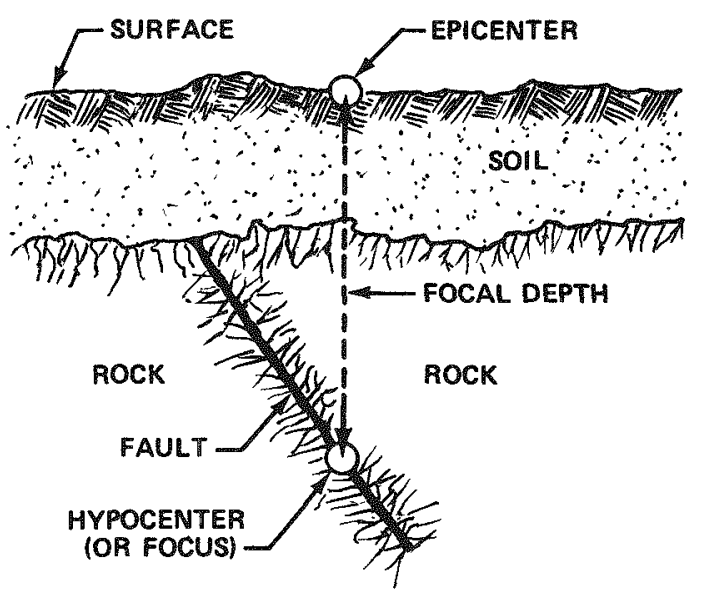

Fig. 6.1 Definitions of earthquake (and related) terms.

epicenter and the hypocenter (or focus), that is to say, the depth of the hypocenter below the surface, is called the "focal depth" of the earthquake.

\section{SEISMIC EFFECTS OF UNDERGROUND DETONATIONS}

\section{Fault Displacements}

In Chapter 4 it was stated that surface fractures and cracks along (or close to) pre-existing fault lines are often produced by underground nuclear detonations (see Fig. 4.3). For devices of high (or fairly high) energy yield, measurable permanent displacements of nearby faults have been observed in both vertical and horizontal directions. The largest of such displacements recorded were for the Faultless calibration test in January 1968 at Hot Creek Valley, Central Nevada (see Fig. 1.1), where there are several known faults; the maximum vertical and horizontal displacements were 15 feet and 3 feet, respectively. As a general rule, however, the displacements are less than a foot. Displacements along faults, although not con- 
tinuous, may extend for a distance of several miles. For the same (or similar) conditions, the linear extent of fault displacement is roughly proportional to the yield of the explosion.

The Benham device with an energy yield of about 1.1 megatons was detonated under Pahute Mesa at the Nevada Test Site (see Fig. 1.2) in December 1968. The locations and vertical displacements (in feet) of surface faulting observed after the event are shown in Fig. 6.2. The thin lines indicate the positions of known (pre-existing) faults whereas the thick lines show where the displacements occurred as a result of the explosion.

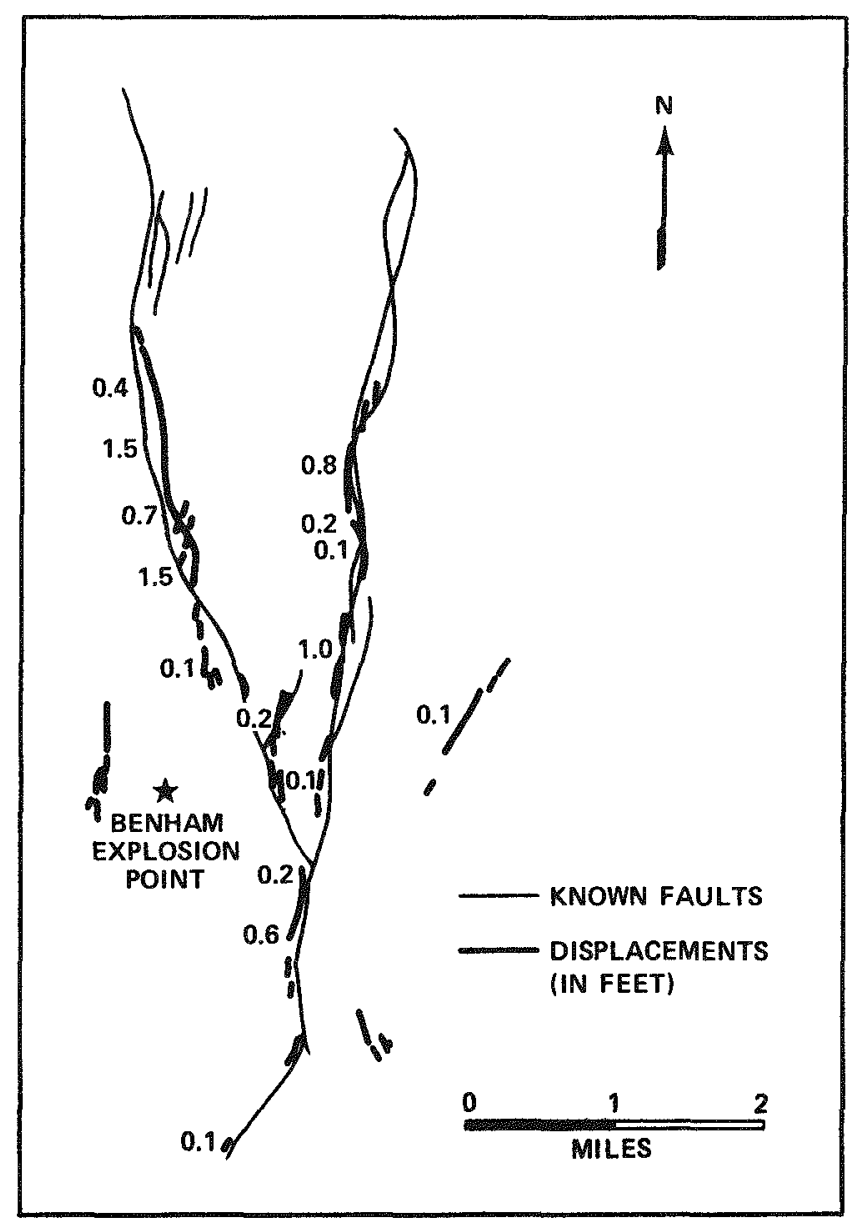

Fig. 6.2 Vertical displacements observed on Pahute Mesa after the Benham test. 
It is clear from Fig. 6.2 that most of the displacements in the vicinity of the Benham explosion point were along pre-existing fault lines. The maximum permanent vertical displacement was 1.5 feet, observed at places 1.5 to 2.5 miles north of the detonation point. Surface fractures occurred over a total distance of some 5 miles north and south.

Previously, in April 1968, the Boxcar device, with a yield similar to that of Benham, had been exploded underground in the same general area. The Boxcar detonation caused fractures along preexisting faults at distances of about 4 miles. Although a displacement of 3 feet was observed at one point within 1 mile of the Boxcar site, vertical displacements were generally less than 1 foot.

Since the Benham event, the Jorum event of somewhat less than a megaton and the Handley event of slightly more than a megaton yield have been conducted on Pahute Mesa. Jorum, which was located about 1.5 miles north of Boxcar, caused additional movement on some faults which had been affected by the Boxcar explosion. The Handley device, detonated some 4 miles west of Boxcar, caused fault displacements of a magnitude comparable to Jorum but not on the same structures.

The Milrow calibration test, with an energy yield of about a megaton TNT equivalent, was made at Amchitka Island, Alaska (see Fig. 1.3) on October 2, 1969. This was the first high-yield detonation on the island. A vertical displacement of 1.5 feet along a 1500-foot lineament was observed in the tundra at a surface distance of 2300 feet southeast of the explosion area. A small fracture was also seen in a fault on the intertidal bench, approximately 5000 feet west of the detonation point; the maximum vertical displacement was less than a foot.

A rough "rule of thumb" has been developed from observations at the Nevada Test Site. According to the "rule," displacement along a fault line may occur only if the surface distance (in feet) from above the explosion point is less than about 1000 times the cube root of the energy expressed in kilotons of TNT equivalent. Thus, for a 1-megaton (1000-kiloton) detonation, displacement would be expected only if the fault were within a distance of roughly $1000 \times \sqrt[3]{1000}=1000 \times 10=10,000$ feet (about 2 miles). In other words, faults that are nowhere closer than 2 miles from a 1-megaton explosion would not be significantly affected. However, if any part of the fault is within 2 miles of the detonation site, the actual 
displacement may be observed along that fault at a greater distance (see Fig. 6.2).

Photographs taken at the time of underground detonations show that the fault movement occurs within the first few seconds after the explosion. Since the displacement is invariably within a few miles of the detonation site, no person or (inhabited) structure will be close enough at the time of a high-yield test to be affected. Moreover, although the fault movement undoubtedly produces seismic waves at a distance, they are weaker than those caused directly by the explosion. If the ground motion from the latter is not a hazard, then that from the former will certainly not be. Hence, fault displacements are not currently considered to be a safety problem.

Further understanding of the cause and effect of fault displacement is being sought. Several experimental programs, involving measurements of earth strains and motions before and after detonations, have been implemented to provide the desired information.

\section{Frequency and Locations of Aftershocks}

Aftershocks that continued beyond the period of chimney growth were observed following the Faultless and Boxcar events in Nevada. In order to make a more detailed study of aftershock phenomena, the U.S. Atomic Energy Commission established a permanent network of seismograph stations on or near Pahute Mesa in preparation for the Benham and subsequent events. This network is generally augmented by temporary stations at the time of a test. The stations have been operated by the U.S. Coast and Geodetic Survey (now the National Ocean Survey of the National Oceanic and Atmospheric Administration) and by the U. S. Geological Survey: National Center for Earthquake Research.

In the 12 days preceding the Benham event eight earthquakes were recorded by the seismograph network; of these, three were minor and five were extremely small. During a period of six weeks following the detonation, some 10,000 aftershocks were detected, all within a few miles of the explosion point. The initial rate was roughly 1000 per day and this fell off, with an occasional increase, to about five per day at the end of the period. A few minor earth tremors were detected a long time after the high aftershock activity stimulated by the Benham explosion had ceased, but their origin is uncertain. 
From the thousands of aftershocks recorded, 640 were chosen for detailed study by scientists of the U.S. Geological Survey to delineate the zone of seismic activity around the Benham explosion point. The locations of the epicenters, as determined from the seismograph signals, are given in Fig. 6.3. The thin lines indicate the

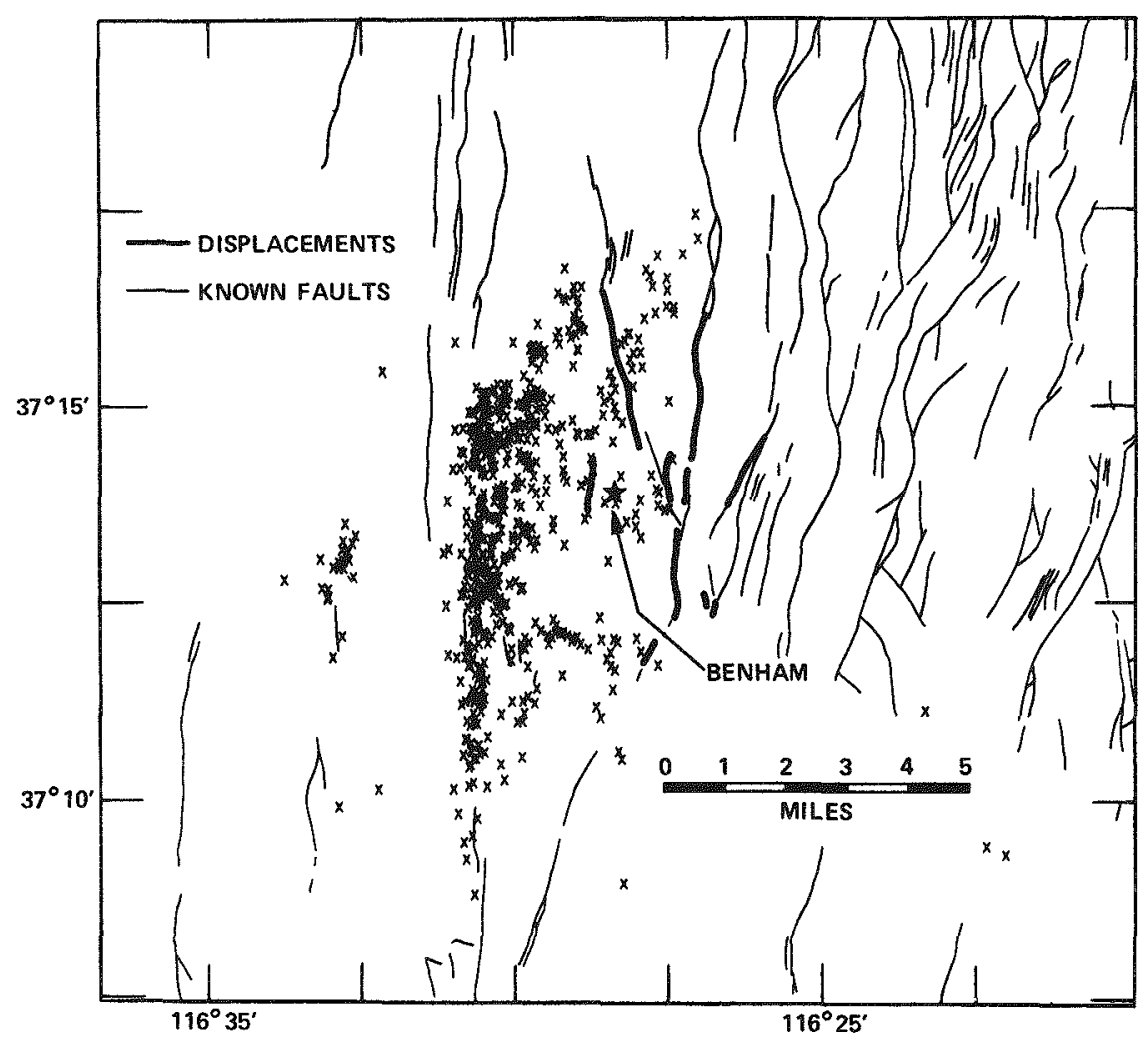

Fig. 6.3 Locations of 640 aftershocks from the Benham test.

positions of known faults and the thick lines show approximately where fault displacements were observed (as in Fig. 6.2) immediately after the detonation.

All but three of the 640 aftershocks had their epicenters within a radius of some 8 miles from the explosion point. The three events located outside this area, at distances of roughly 21 miles away, may not have been related to the Benham event.

It can be seen from Fig. 6.3 that a large number of the aftershocks had their epicenters along a linear region, running north 
and south and passing within about 2 miles west of the explosion point. Several small faults were found to coincide with this zone of activity. Geologists think that these minor faults may be the surface expression of a buried discontinuity along which the aftershocks occurred.

An interesting point about the location of the epicenters of the Benham aftershocks is that only a small number were close to the faults that had suffered significant surface displacement at the time of the detonation (Fig. 6.2). There is evidently a difference between the phenomena of immediate displacement and the occurrence of aftershocks. Both are, however, thought to result primarily from the release by the nuclear detonation of pre-existing natural strains within the rock.

The focal depths (see Fig. 6.1) of the aftershocks following the Benham event were found to range from almost zero (i.e., hypocenter near the surface) to nearly 4 miles. Most of the hypocenters were at depths of 0.6 to 3 miles, lying in a layer of older volcanic rocks. Natural earthquakes in Nevada, however, usually have greater focal depths. The significance of the depth distribution of the hypocenters of the aftershocks following the Benham detonation is not yet known, but studies of the subject are being continued.

The aftershocks (a few hundred) from the Jorum event were much less than from the Benham test in the same general area. Most of the epicenters were within a distance of 2.5 miles from the detonation point and fell generally into two groups. One group clustered around the cavity, whereas the other followed and extended a linear pattern first observed in the northeastern sector of the Benham aftershocks. At 10 days after Jorum, the seismic activity in the area was roughly the same as before the test.

The epicenter pattern of the Handley aftershocks showed a main cluster about 1.5 miles west from the explosion site and a diffuse trend extending some of the epicenters associated with the Benham event along a line about 8 miles southward. There was also a scattered distribution of epicenters east of the Handley location where Benham and Jorum aftershocks had been observed. The total number of aftershocks from Handley was between those for the Benham and Jorum tests.

The aftershocks from the high-yield Milrow test on Amchitka Island are of special interest. This island is located in one of the earth's most seismically active regions and some fears had been expressed that a high-yield explosion might initiate a significant 
earthquake. A previous test (Long Shot) on Amchitka Island in October 1965 was of lower yield (about 80 kilotons TNT equivalent) and it had not been instrumented to detect local seismic effects.

After the Milrow detonation, a few hundred aftershocks of very shallow (about 1 mile) focus were recorded. All of the identifiable aftershock activity following Milrow ceased abruptly when the explosion cavity collapsed 37 hours after the detonation. The energy of the largest aftershocks was less than 1 percent of the seismic energy of the explosion.

The epicenters of the Milrow aftershocks were all within a surface distance of a little more than 3 miles from the detonation area. The pattern of the aftershocks extended to the west in the same general direction as known faults that cut across Amchitka Island (Fig. 6.4). This, together with the shallowness of the hypocenters, suggests that the aftershocks were related to the adjustment of pre-existing strains around these relatively shallow faults.

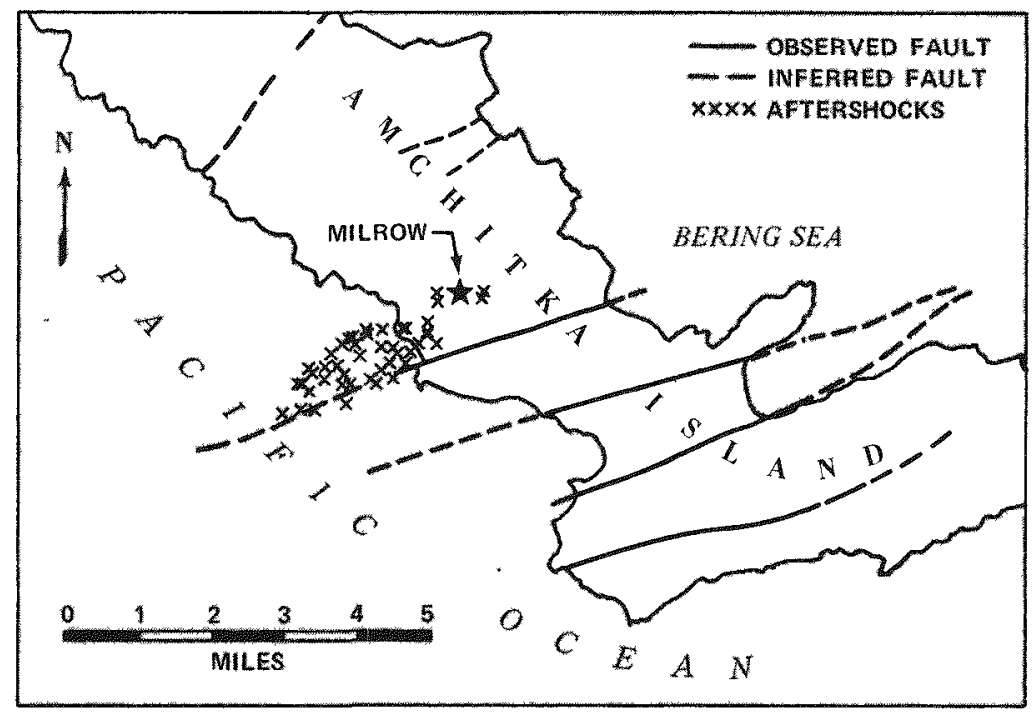

Fig. 6.4 Locations of aftershocks from the Milrow test.

There was no indication of disturbance near the deeper faults (12 to 25 miles) where motion on major structural blocks might take place. If a large earthquake were to occur, it would be associated with motion of this type. Thus, the observed seismic effects of the Milrow explosion gave no indication of any sensitivity to the 
initiation of a dangerous earthquake in the Aleutians by an underground nuclear detonation on Amchitka Island.

\section{More Distant Seismic Effects}

The observations of seismic effects of underground explosions beyond the immediate area of the detonation fall into two main categories. One is concerned with the measurement of temporary or permanent ground strains at various distances up to a few hundred or so miles. Strain meters have been installed, both in Nevada and the Aleutians, to measure the amount of ground distortion resulting from the explosions and also to observe the strain changes due to natural causes during the periods between tests. Familiarity with the strain effects of underground explosions is an important factor in the general understanding of interactions with the earth's crust that might cause the initiation of distant earthquakes.

The strain data show a pattern about as expected, with large strain effects close to the explosion site but a rapid decrease with distance. At distances from 50 to 100 miles the effect of an underground detonation is no greater than the periodic deformations caused by the gravitational fields of the sun and moon (earth tides). Observations of earth strain between tests serve as a basis for comparison with detonation effects. They are also being used to determine if earth strains might provide an indication of early warning of natural earthquakes. This effort is still in too early a stage to have yielded any important conclusions.

Since earth strains may have a direct bearing on the relation between underground detonations and seismic phenomena, the program for the measurement of such strains has been expanded. Organizations taking part in strain studies include the California Institute of Technology, the Colorado School of Mines, the University of Nevada, the University of Utah, the Earthquake Mechanism Laboratory of the National Oceanic and Atmospheric Administration, and the U. S. Geological Survey: National Center for Earthquake Research.

The second type of study of distant effects of underground nuclear explosions makes use of statistical analyses of the frequency of seismic events to seek indications of changes (if any) in the natural rate of earthquake occurrence over a large area. The results of an examination by the National Center for Earthquake Research of 
existing earthquake records are of special interest. A circular region with a radius of 535 miles around the Nevada Test Site, but excluding a small rectangular area about the Site itself, was selected and the numbers of earthquakes were counted for equal time periods before and after 235 underground explosions from September 15, 1961 through December 19, 1968, the date of the Benham event. The total numbers of earthquakes recorded in the 104-hour periods before and after all these detonations were 620 and 616 , respectively. Thus, there appears to be no correlation between underground nuclear explosions and natural earthquakes in the area under consideration.

Other statistical studies of earthquake occurrences before and after nuclear detonations were made for several high-yield explosions by establishing seismograph stations at locations as far as 200 miles away. Some sites were selected because of the relatively frequent occurrence of fairly small earthquakes nearby; if there were any distant effects of underground explosions, it would seem most likely that they would be observed at such places. Other stations were situated where the sensitivity and accuracy for the determination of the size and location of possible earthquakes would be optimum. In no case were there significant variations in the frequencies of detected earthquakes that could be attributed to the Benham event or to any subsequent test.

\section{WATER WAVE EFFECTS}

\section{Formation of Tsunamis}

Tsunami, pronounced tsoo-nah'-me, is a Japanese word meaning literally a tidal wave, but a "tsunami" is not a tide-related phenomenon. It is actually a train of traveling waves generated in the ocean by a large-scale submarine disturbance. Such a disturbance can apparently arise only from a substantial vertical displacement of the sea floor as the result of a major earthquake. The disturbance starts a large oscillation at the ocean surface and the long, low waves produced move away from the source. A landslide either below or above the water surface can cause significant waves, but not a major tsunami.

Tsunami waves are estimated to be about a foot or less in height in the open ocean; they have long periods $(5$ minutes or more 
between the passage of successive waves), and they span a long distance (usually over 100 miles). The speed of propagation depends on the ocean depth, but it is commonly in excess of 500 miles per hour.

Because of their small height and great length, tsunami waves have essentially no effect on ships at sea. The waves are destructive only when they reach a shoreline, where the decreasing water depth can produce an enormous amplification of the wave height. The extent of the enhancement in wave height depends mainly on the configuration of the local ocean bottom near the shore and on the direction from which the tsunami wave approaches. Thus, some particular coastal regions have been found to be much more susceptible than others to inundation by tsunamis.

The records show that a wave height of 93 feet was observed on the island of Miyako, between Formosa and Japan, in 1896. It is evident, therefore, that tsunamis are capable of causing considerable damage by flooding. There is a rough correlation between the magnitude of the underwater disturbance and the destructive potential of the tsunami. But since circumstances are usually different, disturbances of equal magnitude do not always produce equivalent tsunami effects.

\section{Tsunamis and Submarine Earthquakes}

Nearly all observed tsunamis, and certainly all the major ones, are associated with large submarine earthquakes. The principal mechanism of generation, as stated above, is thought to be a large vertical displacement of the sea floor. Because of the inaccessibility of the source region, the exact details of tsunami formation are difficult to elucidate. Data collected over many years show that potentially destructive tsunamis are to be expected only from very strong earthquakes. Furthermore, the focal depth is not likely to be more than about 30 miles. As a general rule for earthquakes of equal magnitude, the shallower the earthquake hypocenter (or focus), the larger the resulting tsunami.

The area in which tsunamis are generated coincides with the region of seismic activity; this includes the whole area where aftershocks are experienced. The dimensions of the aftershock area appear to be directly related to the length of the fault over which the motion responsible for the earthquake has occurred. It has been 
estimated that fault displacement over a length of at least 70 miles is required to generate a potentially damaging tsunami.

\section{Underground Nuclear Detonations and Tsunamis}

There are several ways whereby an underground nuclear explosion on an island, such as Amchitka Island, might conceivably produce a tsunami. First, those that seem to be of minor importance will be mentioned. Then consideration will be given to a mechanism that appears to be more plausible.

The view has been held, although not commonly at present, that a tsunami can be initiated by a submarine landslide (or slump) in an area where there is much sedimentation and little seismic activity. Sediments would then be unstable and a moderate disturbance might cause a considerable slide. An underground nuclear detonation might generate a tsunami in this manner. In the region of Amchitka Island, there is little unstable sediment and the area is seismically active. A major submarine slump would seem to be out of the question and the worst expected effect of a nuclear explosion in this regard would be localized wave formation.

The suggestion has been made that seismic waves traveling along the surface, rather than through the body of the earth, could interact (or couple) with water waves and transfer energy to the latter. This coupling mechanism is considered to be unlikely because seismic waves travel faster than water waves. In any event, in underground nuclear explosions a smaller proportion of the energy goes into seismic surface waves than in an earthquake of equivalent magnitude.

Now a more serious possibility will be examined. If an underground nuclear detonation should initiate a major submarine disturbance, a tsunami might occur. As was noted earlier, only a very strong earthquake would be capable of generating a tsunami. Calculations have shown that no proposed detonation would produce a submarine disturbance of the required magnitude, either as a direct effect or as a result of the aftershocks. Actual measurements have provided general confirmation of these calculations.

Suppose, however, that the unexpected happened and a nuclear explosion on Amchitka Island did trigger a major earthquake. Such an earthquake, which would almost certainly be in the western Aleutians, appears unlikely to produce a significant tsunami. This opinion is based on the fact that there is no historical record of a 
damaging tsunami having been generated by any earthquake in the Aleutian Islands west of the Amchitka Pass, which includes Amchitka Island, although many earthquakes have occurred in this region. The reason is apparently related to a characteristic absence of significant vertical displacement from earthquakes in the western Aleutians.

On the whole, taking all the known facts into consideration, the conclusion was reached that underground nuclear tests on Amchitka Island would produce only minor wave effects localized near the test area. Instruments placed offshore to measure permanent ground displacements in about 100 feet of water showed no detectable change after the Milrow test (p. 125). Furthermore, deep-sea gauges near Amchitka Island recorded no waves associated with the explosion above the normal background variations of the instruments, which were about half an inch.

A program of theoretical and experimental research on tsunami propagation has been under way for some time (p. 222). This program will be continued as long as underground nuclear explosions are planned for Amchitka Island. In this way, the understanding of tsunamis and their possible generation by such detonations will be improved.

\section{Formation of Seiches}

Like a structure, a lake, bay, or other body of water that is almost (or completely) landlocked has a natural period of oscillation (see Chapter 5). As a result of resonance, severe shaking of the earth can set the water into its natural oscillation which may last for days. An up-and-down surface oscillation of this kind is called a "seiche," pronounced saysh. Strong earthquakes are known to produce seiches, and sometimes a seiche results from the arrival of a tsunami in a bay.

Since seiches have the potential for causing damage by flooding, the possibility that an underground nuclear explosion could generate a seiche must be considered. The only bodies of water where this could happen are lakes or reservoirs impounded by dams fairly near the explosion point. As far as the Nevada Test Site is concerned, the most important lake is Lake Mead, behind Hoover Dam; this dam is about a hundred miles from the center of the Nevada Test Site.

Of the many earthquakes that have occurred around and under Lake Mead, none has been known to cause a significant seiche. The 
motions of Hoover Dam accompanying these earthquakes have been about a hundred times greater than those produced by the underground explosions in Nevada. On the basis of observations and calculations, there is no probability that nuclear detonations might induce significant seiches in Lake Mead. The ground motion amplitudes are too small and the periods too short to develop resonances in the lake.

It was mentioned on page 113 that before the Rulison event in Colorado, the possibility was considered that ground motion from the explosion might cause some damage to earthen dams in the area. At the same time, a study was made of the generation of seiches in the reservoirs. This study indicated that the Rulison detonation would not cause seiches to develop and measurements confirmed this expectation. Similar studies would be made in any area in which a nuclear detonation is planned to make sure that no seiche would result.

\section{SUMMARY}

Surface fractures and cracks along pre-existing fault lines are of ten produced by underground nuclear detonations. For devices of high (or fairly high) energy yields, permanent surface displacements of a foot or more in both horizontal and vertical directions have been observed in nearby faults.

The largest underground explosions, like earthquakes, are followed by aftershocks. The energy of the strongest of such aftershocks has so far never been more than a few percent of the seismic energy generated by the detonation, but in the great majority of cases the aftershock energy has been even smaller. For explosions of high energy yield, aftershocks may continue, although at a greatly reduced rate, for many days after the underground test. Very few of such aftershocks have originated more than about 12 miles from the explosion point. For the high-yield Milrow calibration test on Amchitka Island, Alaska, the aftershocks ceased abruptly when the explosion cavity collapsed.

Fault displacements and aftershocks caused by an underground explosion apparently result from the release of natural strain (deformation) in pre-existing faults. Neither has represented a hazard to people or to structures off the Test Sites. Records of earthquakes 
in the western United States show no correlation between underground nuclear detonations and the frequency of natural earthquakes.

A strong earthquake may produce a tsunami wave system in the ocean that can, in certain circumstances, cause flooding at distant shorelines. It is not expected that any contemplated nuclear detonation on Amchitka Island would cause an underwater displacement sufficiently large to generate a tsunami. There is no reasonable possibility of the formation of a seiche in connection with any underground detonation, either past or future, at the Nevada Test Site. 


\section{Chapter 7 \\ METEOROLOGY AND \\ RADIATION PREDICTIONS}

\section{INTRODUCTION}

\section{The Meteorological Program}

In an underground nuclear detonation, at a depth of burial appropriate to complete containment for the expected energy yield (Chapter 3 ), the probability that a significant amount of radioactivity will escape into the atmosphere is small. Nevertheless, in the interest of safety, the possibility of an accidental release of radioactivity is always taken into consideration in planning and conducting an underground nuclear event. Consequently, an extensive "radiological safety" program, about which more will be said in the next chapter, has been established for the purpose of minimizing all possible effects that might arise from radiations associated with nuclear detonations.

The fate of the radioactive material that may escape from the site of an underground nuclear explosion depends to a large extent on the atmospheric conditions. Hence, meteorological support is required to provide for the evaluation of these conditions and their influence on the transport and diffusion of any radioactive residues that might escape into the atmosphere. Such support is an essential aspect of the radiological safety program. In this chapter, the importance of meteorology in connection with underground nuclear detonations will be considered.

Meteorological support for the nuclear explosions conducted by the U. S. Atomic Energy Commission is provided by the Air 
Resources Laboratory, Las Vegas, Nevada, of the National Oceanic and Atmospheric Administration (formerly the Environmental Science Services Administration). The program has two main and related aspects. One is to provide assurance, prior to a test, that if there is an accidental escape of radioactivity, the material will be carried by the wind away from any populated areas in the vicinity. The second is to estimate, as far as possible, what the total radiation exposures might be at various distances downwind from the explosion site in the event that an escape of radioactivity should occur. Since there are always people somewhere downwind, the radiation must not represent a hazard to these people.

The chief properties of the atmosphere that affect the movement of any released radioactive material and consequently influence safety decisions are the wind speed and direction, mixing (turbulence) within the atmosphere, and possible precipitation. The measurement and importance of these factors are described in the next section (p. 140).

Observations of wind speeds and directions, of air temperature and pressure, and of precipitation are obtained from the extensive network of the National Weather Service, as well as from a supplementary network in the vicinity of the detonation site. From the data, the conditions of the atmosphere at the planned time of the explosion are predicted.

For the Nevada area, typical weather patterns have been discerned from the large amount of information that has been collected over the past years. These have been very helpful in improving the accuracy of the predictions and thus in determining the best time for each test to be made. For example, it is known that there is a pronounced tendency for the winds near the surface over Yucca Flat (see Fig. 1.2) to be northerly (i.e., from the north) at night, shifting through east to southerly (i.e., from the south) during the day, to southwesterly in the late afternoon. The changeover from northerly to southerly winds occurs earlier in the day in the summer and later in the winter.

If a detonation is to be performed in a new area, a study is first made of climatological data obtained from weather archives and from an observation program. These indicate the frequency of occurrence and the predictability of conditions that are favorable, as well as unfavorable, for the planned nuclear event.

In contained Plowshare experiments outside the Nevada Test Site, the explosion depth has been much greater than the minimum 
depth of burial required to prevent the seepage of radioactive gases. Consequently, the probability of the release of radioactivity to the atmosphere has been extremely small. Nevertheless, in the interest of safety, strict weather precautions have always been observed. As more experience is gained with very deep underground detonations, the meteorological program for such explosions may be modified appropriately.

\section{Determination of Detonation Time}

No matter what the locality, underground nuclear tests are conducted only under weather conditions that meet safety requirements. To aid in making final preparations, weather briefings have been held on the afternoon of the day preceding that scheduled for the explosion, and again two or three hours before the planned detonation time. If wind and weather conditions are acceptable, a final schedule is determined.

If the conditions are not acceptable, the detonation is rescheduled, perhaps by short time increments, until suitable weather is observed in a persistent or regular pattern. But if the immediate situation does not appear to be favorable, the nuclear detonation is postponed to a later date to allow for a major change. Moreover, any planned explosion can be cancelled or delayed up to within a second (or so) of the scheduled detonation time. Thus, late changes in the weather can cause, and sometimes have caused, a last-minute postponement.

\section{Meteorology After the Detonation}

If an accidental venting or seepage of radioactivity were to occur, meteorological conditions would be observed for some time after the detonation. In the unlikely event that a radioactive cloud should form, its motion and the possible associated fallout can thus be predicted and confirmed by observations from aircraft and by ground monitors. Appropriate action would be taken to prevent radiation exposures that might approach the accepted guideline limits described in Chapter 8.

When an underground explosion is used to stimulate the release of natural gas in "tight" rock formations, the effects on the gas reservoir are evaluated by controlled withdrawal of gas at different 
rates from the rubble chimney. This gas has been "flared," that is, burned, at the top of a tall stack. The combustion products then contain some radioactive tritium, as tritiated water vapor (p. 29), and the fission product gas krypton-85. The concentrations of these substances, which decrease as flaring proceeds, have been monitored continuously, and at the same time the meteorological conditions have been observed. Flaring can be stopped at any time, by merely closing valves, if any combination of radioactivity and weather should indicate that this is desirable.

\section{METEOROLOGICAL MEASUREMENTS FOR THE NEVADA TEST SITE}

\section{Wind Speeds and Directions}

Large-scale wind data in the general area of the explosion site-the western United States for the Nevada Test Site-are obtained from the National Meteorological Center, Suitland, Maryland. At this center, surface and upper-air observations from a large network of fixed weather stations and from satellites are collected and analyzed. From the results, the large-scale pattern of air motion at any particular time can be established. Figure 7.1 is an example of an instantaneous representation of the streamline air motion at an altitude of 10,000 feet above mean sea level over the western United States. In this case, the air motion over Nevada is toward the northeast.

The large-scale streamlines of air motion indicate the general direction in which escaping radioactive material would be carried from an underground nuclear detonation. To provide more detailed information, however, these streamlines must be supplemented by local observations. Thus, wind speeds and directions near the surface are measured regularly at some fifteen stations distributed over and near the Nevada Test Site. In addition, wind speeds and directions above the surface are obtained from a temporary local network of stations established before each event. Rising balloons are tracked optically, by radar, or by radio signals transmitted from instruments on the balloons (Fig. 7.2). From the motion of the balloons, the winds at different altitudes and locations can be determined. 


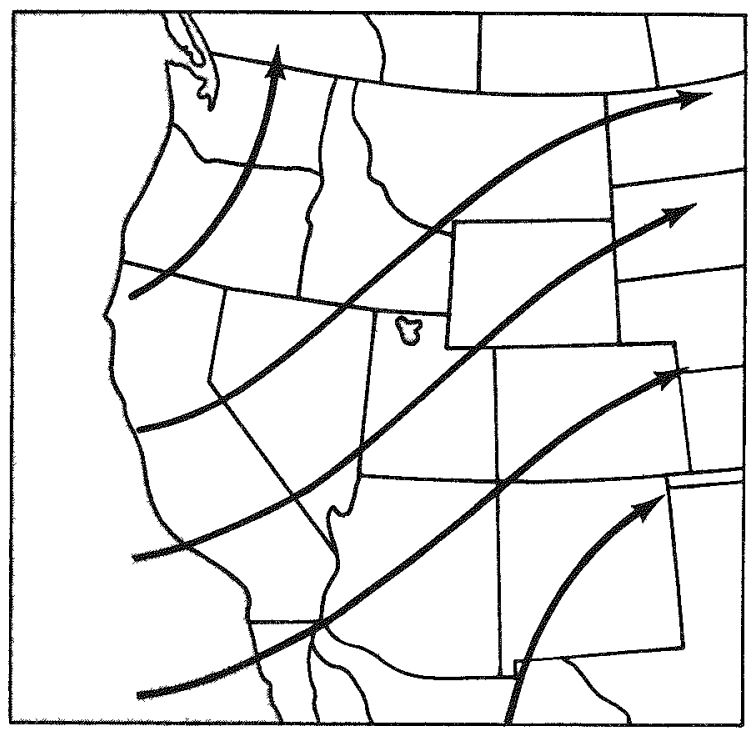

Fig 71 Example of streamline arr motion over the western United States

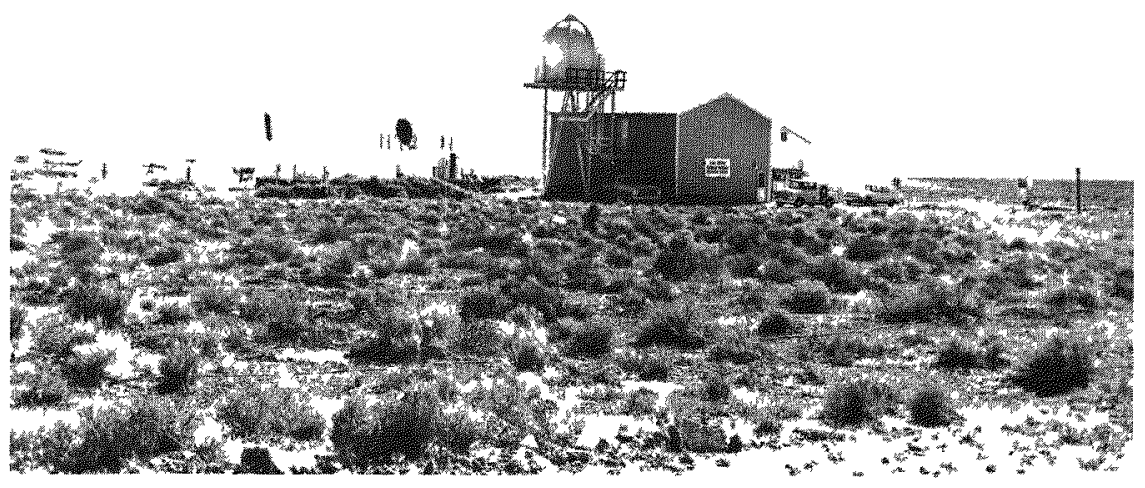

Fig 72 Weather station at the Nevada Test Site showing tracking radars 
Direction and Speed of Wind at Various

Altitudes Above Yucca Flat

\begin{tabular}{ccc}
\hline $\begin{array}{c}\text { Height above } \\
\text { surface (feet) }\end{array}$ & $\begin{array}{c}\text { Direction } \\
\text { (degrees) }\end{array}$ & $\begin{array}{c}\text { Speed (miles } \\
\text { per hour) }\end{array}$ \\
\hline 5000 & 210 & 20 \\
4000 & 200 & 15 \\
3000 & 200 & 10 \\
2000 & 180 & 5 \\
1000 & 170 & 5 \\
- & 10 & 5 \\
\hline
\end{tabular}

The accompanying table provides an example of how the speed and direction of the wind might change with altitude above Yucca Flat at a given time. The direction of $0^{\circ}$ (or $360^{\circ}$ ) indicates air flow from the north, $90^{\circ}$ from the east, $180^{\circ}$ from the south, and $270^{\circ}$ from the west. It can be seen from the table that at the surface the wind is coming almost from the north at a speed of 5 miles per hour. But at 1000 feet above the surface the direction has changed significantly, although the speed is about the same. The wind at this altitude is now blowing almost from due south. At higher levels, the wind becomes more southwesterly but the change in the direction with altitude is much less than near the surface in this particular case. However, the speed increases from 5 to 20 miles per hour. Vertical changes of wind speed and direction, called "wind shear," must be known if the fate of the radioactive material that escapes from an underground nuclear detonation is to be predicted with reasonable accuracy.

Wind speeds and directions change with time, often quite rapidly. One way of displaying the variations is shown in Fig. 7.3; the directions and speeds of the winds at six-hour intervals are indicated at three levels, namely, at the surface and at heights of 1000 and 5000 feet above the surface. Surface winds are measured continuously, but those above the surface are determined by tracking balloons and so are observed only at intervals.

\section{Final Meteorological Support}

The final preparations for a nuclear detonation begin several days ahead. For long-range weather forecasts, much reliance is placed on 

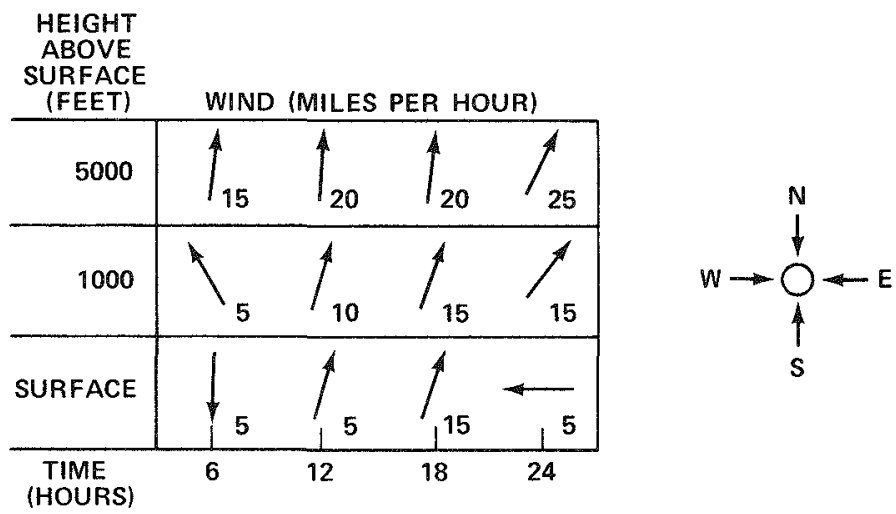

Fig. 7.3 Example of changes in wind speed and direction with time at three levels.

the large-scale prognostic charts prepared by the National Meteorological Center. As the detonation time draws closer, increasing reliance is placed on the skill and experience of local meteorologists in predicting wind conditions to be expected at the detonation site and the surrounding area at the time of the detonation and for a period thereafter. To prepare these forecasts, meteorologists at the Air Resources Laboratory, Las Vegas, make use of their own data analyses and those provided twice daily by the National Meteorological Center. They also utilize relationships between wind and other local circumstances, such as those mentioned earlier, that have been observed over many years. Efforts are continually under way, however, to improve the prediction of local wind characteristics.

During the period of a few hours before the proposed detonation time, when the final safety decisions are being made, special wind measurements are taken at short intervals. They are continued after the explosion until there is no longer any risk that radioactive residues will escape into the atmosphere.

Some of the observations are made by means of instruments mounted on a network of towers on the Nevada Test Site (Fig. 7.4). In addition, pilot balloon measurements, called "pibals" for short, are normally made at stations out to distances between 25 and 30 miles from the explosion point. The rising balloons are tracked optically to provide data on upper wind conditions. Radar is also used at one or more stations to track the balloons, thereby assuring wind data even when the weather is cloudy. These special wind 


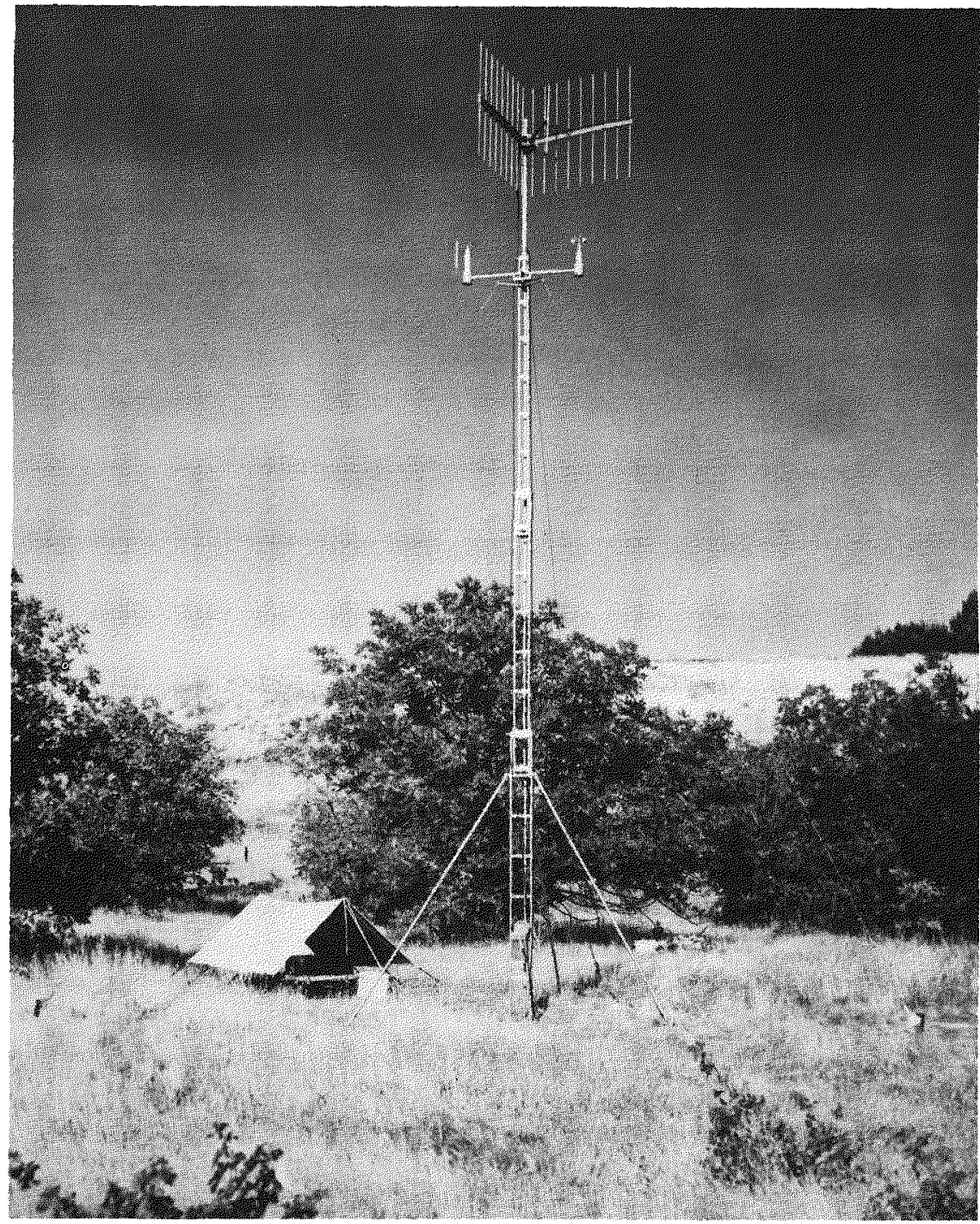

Fig. 7.4 Radiotelemetry station for recording surface winds at the Nevada Test Site.

measurements are taken at hourly or half-hourly intervals up to altitudes of 15,000 to 20,000 feet. The data are transmitted by radio or by telephone to the Control Point for the particular nuclear event and are available within 20 to 30 minutes after release of a balloon. In certain circumstances, such as when the atmospheric conditions are changing rapidly, the number of pibal and other wind- 
measuring stations is increased above that normally in use. Additional stations are located off the Nevada Test Site, especially in the downwind direction, that is, in the direction in which any radioactive material in the atmosphere is expected to move.

\section{Precipitation and Clouds}

Predictions of precipitation make an essential contribution to the meteorological safety aspects of an underground nuclear detonation. If the radioactive matter should encounter a region where precipitation is occurring, then some may be brought to the ground by rain or snow. As a result, there may be local areas where the radioactivity is much higher than would normally be expected in the absence of precipitation. Such areas of higher radioactivity are referred to as "hot spots." At distances beyond the hot spots, the radioactivity would be correspondingly less than otherwise expected. Standard meteorological methods are employed for evaluating present and future moisture cloud distributions. Moreover, in order to provide the best possible information about the probability of precipitation and to identify where such precipitation has occurred, radar installations at Federal Aviation Administration traffic control centers in the western United States are used for weather surveillance. Data about positions of precipitation are sent hourly (or oftener) to the Nevada Test Site. A map of weather distribution, such as that in Fig. 7.5, can then be prepared.

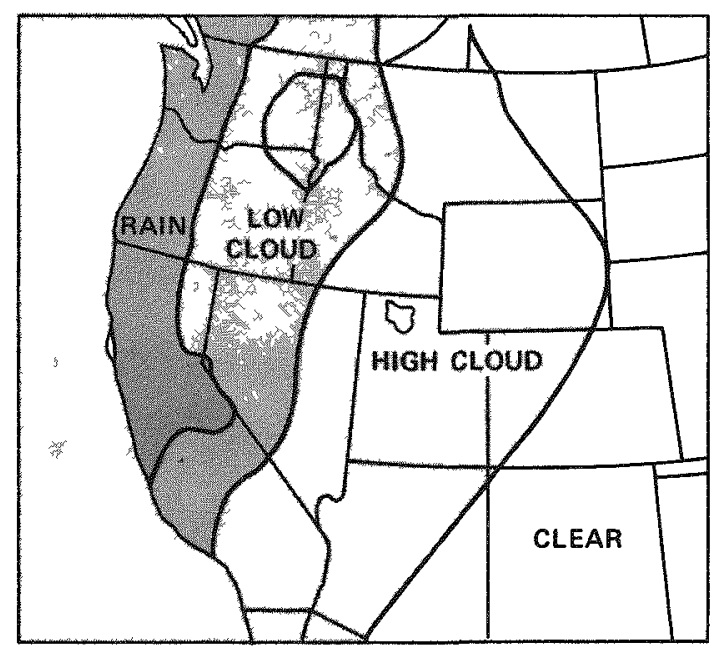

Fig. 7.5 Example of weather distribution in the western United States. 
Precipitation prediction is, however, not just a matter of watching rain conditions move across the country. Rain can form suddenly in an area without a recent history of precipitation. There is, of course, some probability that this may happen in the general vicinity of the Nevada Test Site. Consequently, several studies are being made locally to develop procedures for giving maximum advance warning of rain. Schemes for improving forecasts of spring rains and summer thunderstorms appear to be promising.

Aircraft would play an important role in tracking the movement of radioactive material that might be released to the atmosphere by an underground nuclear detonation. The presence of clouds interferes with this tracking operation. Hence, the most favorable conditions at the Nevada Test Site would include a virtually cloudless sky, especially in the main downwind direction.

\section{Long-Range Trajectories}

In cratering experiments with nuclear explosives (see Appendix), a radioactive cloud is formed and it rises rapidly to a height of many thousand feet. This cloud is then transported away from the detonation site by the winds at the cloud level. In the past, such radioactive clouds have been tracked by aircraft, but the procedure has had its drawbacks. Another technique makes use of plastic balloons, called "tetroons" because of their tetrahedral (four-sided) shape. These balloons are inflated with gas at such pressures as will permit them to float at various preselected altitudes (Fig. 7.6). Tetroons are also a valuable aid in the assessment of long-range trajectories because they can be utilized even in the absence of a radioactive cloud.

The movement of the tetroons at different altitudes is followed by radar. For distances up to 20 or 30 miles, the tetroons are tracked by radar installations based on the Nevada Test Site; for greater distances, up to hundreds of miles from the site, Federal Aviation Administration and U.S. Air Force radars are used. The information so obtained provides fairly reliable data on air parcel trajectories at different altitudes over great distances.

Tetroons have some important advantages over aircraft. The former can be flown within the radioactive cloud, if there is one, and can be tracked continuously. Consequently, tetroons provide tracking at all times of an air mass containing radioactivity. Aircraft, on the other hand, must land occasionally for refueling and to permit 


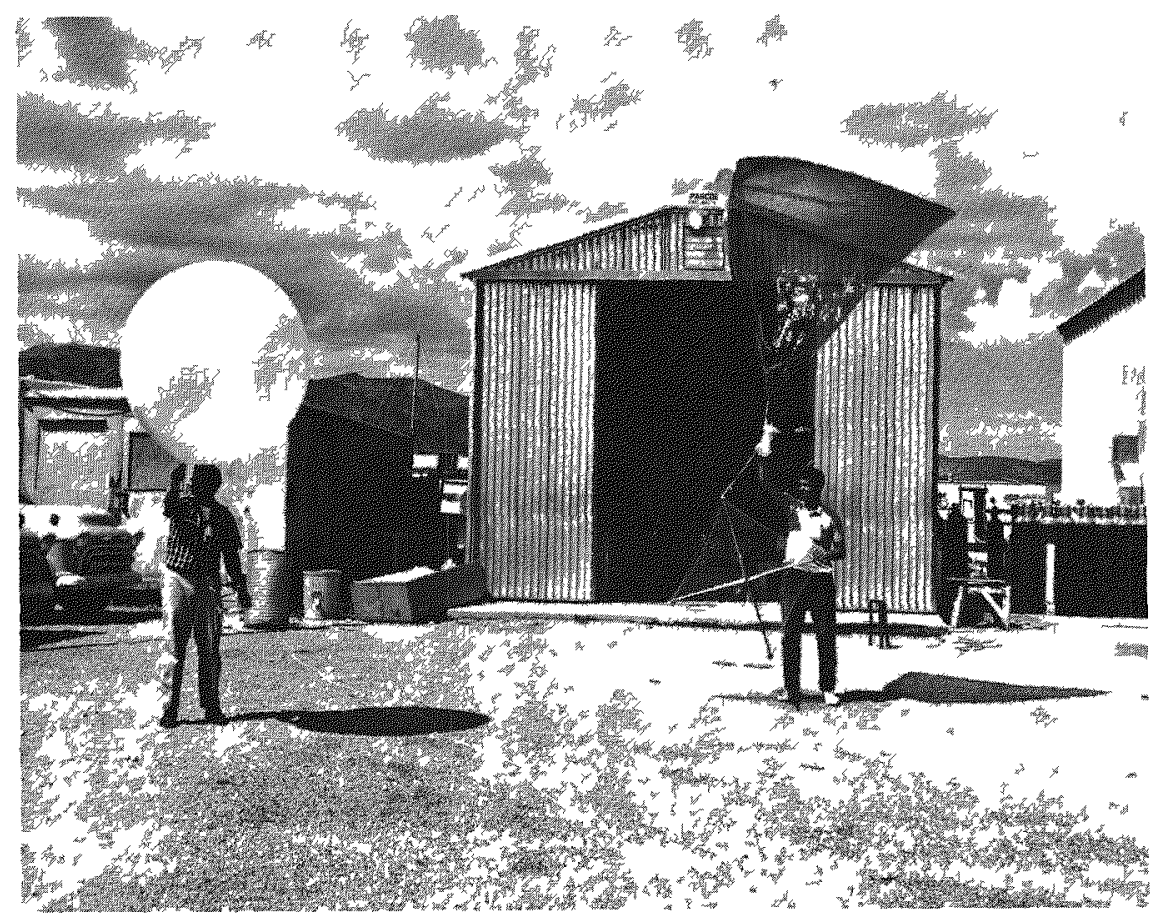

Fig. 7.6 Tetroon with radar reflector attached and tow balloon.

the crew to 1est (or be replaced), furthermore, alrcraft cannot drift with the radioactive cloud as tetroons do

The tetroons have three handicaps they must be flown above mountain ridges in order that they may be detected by distant radars, they do not alwdys follow the motion of the cloud where the main part of the radioactivity is located, and they descend if they get wet or leak gas Tetroons have sometimes been tracked for as long as 24 to 48 hours, but in many cases contact has been lost after shorter times In spite of these drawbacks, tetroons have contributed significantly to the assessment of long-range trajectories of the cloud of debris that may be produced by an underground nuclear detonation

\section{Atmospheric Turbulence}

If a radioactive cloud should be formed, the height to which it ascends would be important in estimating possible radiation exposures Consequently, prediction of the maximum cloud height is an 


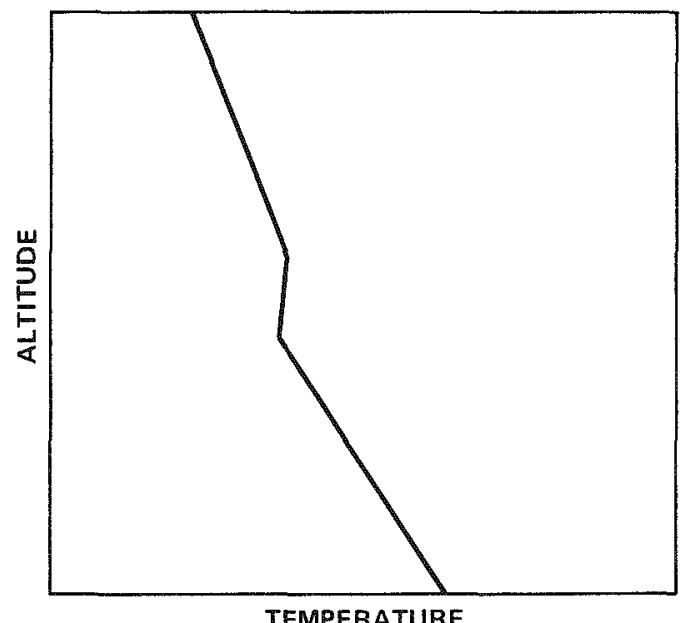

TEMPERATURE

Fig. 7.7 Example of the change of air temperature with altitude.

important aspect of the meteorological program even when a cloud is not expected. Cloud height is determined to a great extent by turbulence in the atmosphere, and this depends in part on the vertical distribution of temperature. Temperature measurements are taken regularly twice daily at Yucca Flat on the Nevada Test Site. Other observations are made when more details are needed of expected changes in the atmosphere. The rate at which the temperature changes with increasing altitude, as depicted graphically in Fig. 7.7, is called the "lapse rate." By using accepted assumptions, meteorologists can then determine the local vertical thermal structure of the atmosphere from relatively few temperature measurements.

A mathematical procedure developed for the study of air pollution has been found useful in estimating the maximum height the cloud might attain. The basic data required for the calculations are the latest available soundings of temperature, the lapse rate, and the daily variation of surface temperature at the location of interest. It happens that, as a result of the frequently cloudless desert, the daily change in surface temperature is one of the most easily predicted meteorological quantities for the Nevada Test Site.

\section{Effect of Prediction Errors}

It is well known that weather conditions cannot always be predicted with great certainty. The reason is that the atmosphere is a 
very complex system and the state of even a relatively small portion at a particular time may be known only imprecisely. Consequently, the radiological safety program based on forecasts of the state of the atmosphere must allow for large deviations from these forecasts.

Errors in predicting the early movement of the radioactive cloud, if one should be formed in an underground nuclear detonation, are of greatest concern. The reason is that plans for clearing operating personnel and equipment from the downwind areas near the explosion site depend on this forecast. Such action must be taken quickly and there would be no time to correct possible prediction errors. It is the standard practice, therefore, to provide a large exclusion area around and immediately downwind of each detonation.

At greater distances from the explosion site, the longer travel time of any airborne radioactive debris would allow clearance of additional areas in case of unexpected changes in the predicted cloud trajectory. The capability of reacting to sudden changes in the atmospheric conditions is facilitated by the continuous monitoring of surface winds and the half-hourly soundings of upper-level winds, as described earlier.

Confirmation that radioactive material, if present, is moving as the predicted flow would suggest or as the measured winds would indicate is provided by aircraft tracking (see Chapter 8). These aircraft, carrying radiation detection instruments, sweep across all areas in which radioactivity could conceivably be present. The data are transmitted to the ground by radio and they provide exact information concerning the local trajectory of the radioactive debris. If there are deviations from the expected pattern, action could be taken for the protection of people and domestic animals in the areas where there might be significant exposure to radiation. An extensive program of radiation monitoring, described in Chapter 8 , would be carried out on the ground in all areas where appreciable radiation exposures are possible.

\section{PREDICTION OF MAXIMUM RADIATION EXPOSURES}

\section{Introduction}

For underground nuclear detonations that are designed to be completely contained, no radioactivity is expected to escape into the 
atmosphere. Although there have been some accidental releases in the past, as a result of seepage or venting (see Chapter 3 ), in the great majority of underground nuclear tests there has been no leakage of radioactivity. Nevertheless, the possibility of an accidental release is provided for on the basis of the worst previous case.

Experience has shown that this is a conservative approach, especially for Plowshare experiments at great depths of burial and when nuclear devices of high yield are detonated at an appropriate scaled depth (p. 54). Studies are therefore being conducted to throw more light on the problem. These studies will result in a better understanding of the mechanisms leading to the escape of radioactive residues from underground explosions. More realistic estimates can then be made of the probable consequences of an accidental release of radioactivity.

\section{The Fallout Sector}

Although no fallout of radioactive particles is expected, and has not often been experienced, from an underground explosion designed for complete containment, it has been the practice at the Nevada Test Site to predict the geographical area over which fallout might occur in the event of the formation of a radioactive cloud. This projected area, which is based on meteorological data, is called the "fallout sector." Since the fallout sector is much more important for nuclear excavation projects than for explosions designed for containment, it is described in the Appendix. The maximum conceivable radiation intensity in the fallout sector is predicted and the results are presented at the weather briefing.

\section{Radiation from Escaping Gases}

If noncondensable radioactive gases escape unexpectedly by seepage from an underground nuclear explosion, the gas is considered to form a vertical column or "plume," like a plume of smoke. The radioactive plume is carried along by the wind and at the same time it spreads upward and outward as a result of turbulence in the atmosphere.

Except possibly for some radioiodine and tritium (as tritiated water), there will be very little radioactive material deposited on the ground as the gas plume passes over a particular area. Nevertheless, people and animals may be exposed to radiation from the plume. However, once the plume has passed, the external radiation exposure 
ceases. The situation in this respect is thus different from that in which solid particles contaminated with radioactivity are left on the ground (see Appendix).

By the use of diffusion theory and the results of field experiments, an equation has been developed from which the radiation dose on the ground from a gas plume can be computed. The calculation takes into account the quantity of radioactive gas released, the wind speed, the distance from the explosion site, and the vertical and lateral (crosswind) spread of the plume.

In the Gasbuggy event (p. 48), the explosive device was emplaced at a scaled depth of burial of over 1400 feet. This is about four times the scaled depth that would normally have been used to assure containment of all radioactive residues for a device of the same energy yield ( 29 kilotons TNT equivalent). For the Rulison experiment (p. 68), the scaled depth of burial was even greater. Accidental venting was consequently deemed to be highly improbable. On the basis of both calculations and previous experience the conclusion was reached that the maximum hypothetical release would be by seepage of radioactive gases. This assumption was then used to predict maximum hypothetical radiation exposures in the vicinity of the explosion site.

There was no detectable radioactive seepage from the Gasbuggy and Rulison detonations. The only release observed immediately after the Gasbuggy event was an insignificant amount of fission product gas that escaped when the electrical cable for firing the nuclear device was cut.

As already noted, the combustion products from the flaring of the natural gas released by the underground nuclear detonation contain radioactive tritium and krypton- 85 . These are primarily beta-particle emitters and the emission of gamma rays is very small. In addition, the effective release height of the flared gas, because of its momentum and buoyancy, is well above the surface. Hence, the radiation exposure on the ground from the plume formed by the flared gas is negligible. The potential effects of the tritium and krypton- 85 when they have become widely dispersed in the atmosphere will be discussed in the next chapter.

\section{WEATHER AND RADIATION BRIEFINGS}

Mention was made earlier of the weather briefings held prior to the scheduled time of a planned nuclear detonation. With the 
background provided in this chapter, the information included in typical weather briefings, which are intended to assure the maximum possible degree of radiological safety, can now be described. The order of presentation of the various meteorological and related data given below are not meant to indicate their relative importance. All aspects of the existing situation are taken into account before a decision is made either to proceed with the detonation or to delay it, possibly for a few hours or perhaps for a day or more. The items enumerated here refer specifically to the Nevada Test Site, where the great majority of nuclear explosions are performed.

1. A broad-scale picture is presented of the predicted mean airflow over the area, as expected at the proposed detonation time. An example of such a picture for the western United States is given in Fig. 7.1.

2. The predicted wind directions and speeds, in 1000-foot layers over the proposed explosion site at the detonation time, are displayed, as in the tabulation on page 142.

3. Since the wind will not remain constant, the predicted variations of speed and direction with time for the postdetonation period of interest are presented. The data are similar to those shown in Fig. 7.3.

4. In order to predict the height that would be attained by a radioactive cloud, should one be formed, the variation of the atmospheric temperature with height is described (see Fig. 7.7). Two or more curves of air temperature versus height may be given in order to indicate the changes that may be expected with time.

5. The predicted long-range trajectory, for two or more different levels in the radioactive cloud, is presented.

6. The local geographical area that may be subject to fallout is projected as a fallout sector (see Fig. A.6). If the cloud is not expected to rise very high, so that it is transported by essentially the same wind at all levels, the fallout will be restricted to a fairly narrow band in the predicted wind direction.

7. The calculated maximum radiation intensity expected along the centerline of the fallout sector is presented to supplement the information in Item 6 (see Fig. A.9).

8. In order to determine the possibility that radioactive material in the atmosphere may encounter precipitation as it travels downwind, the briefing would include a map, such as that in Fig. 7.5, showing areas having different kinds of weather. 
For Plowshare events in which the nuclear explosive is buried at very great depths, the chances that any significant amount of radioactivity will escape into the atmosphere are virtually nonexistent. It may then be possible to modify the meteorological program and the weather and radiation briefings described above without affecting the safety of the operation.

\section{SUMMARY}

In an underground nuclear detonation designed for containment, the probability of the escape of radioactivity into the atmosphere is small. Nevertheless, precautions are taken to assure that should an accidental release occur it would not represent a hazard. The fate of the radioactive material would depend mainly on wind speeds and directions from ground level to high altitudes and over considerable distances. Consequently, an extensive meteorological program is conducted as an aspect of radiological safety.

In making final preparations for an underground test at the Nevada Test Site, weather and radiation briefings are held on the day preceding the event and again about two or three hours before the scheduled time for the detonation. At these briefings, the presentations include the latest meteorological information and predictions of the worst anticipated distribution of radiation exposures that could result from the planned explosion. Unless the conditions are deemed to be satisfactory, the event is postponed until such conditions are established and are expected to continue. In the interest of safety, this procedure has been used even if the probability of the release of radioactive material to the atmosphere is extremely small. For explosions at very great depths, modifications may be permissible without affecting the safety of the operation.

After the nuclear detonation has been carried out, a program of radiation monitoring, both from the air and ground, would be instituted if necessary. The nature of this program and the actions taken to protect people and domestic animals from the effects of radioactive material, should any escape, are described in the next chapter. 


\section{Chapter 8 \\ THE RADIOLOGICAL SAFETY PROGRAM}

INTRODUCTION

The basic purpose of the radiological safety program is to ensure that public health and safety are protected from radioactivity resulting from underground nuclear detonations, no matter where they are performed. Even when the nuclear explosive is buried at a depth such that containment of the radioactivity is virtually certain, the program is designed to deal with any possible release of radioactivity to the biosphere.

Some of the important objectives of the radiological program, based on an understanding between the U.S. Atomic Energy Commission and the U. S. Public Health Service, have been stated as follows:

1. To verify the off-site (i.e., outside the controlled access area) radiological situation associated with tests to ensure protection of the public from radiological (and other) effects of nuclear testing. In the event unacceptable situations develop, to effectuate measures as prescribed by the Atomic Energy Commission.

2. To document through radiation monitoring and environmental surveillance the radiation exposures to off-site areas.

3 . To assure the public through personal contact, and a program of community relations and public education, that all reasonable safeguards are being employed to protect their health (and property) from the effects of testing. 
4. To document any increase of environmental levels of radioactivity due to nuclear testing.

5. To conduct special studies to determine the transport of radioiodine in environmental and biological systems and to determine its effects on man.

\section{RADIATION PROTECTION STANDARDS}

\section{Exposure to Radiation}

It has been known for many years that exposure to radiations, such as $\mathrm{X}$ rays and radiations emitted by radioactive substances, may be harmful to man. However, all human beings (and animals) are continuously exposed to low levels of radiation from natural sources. These sources of what is called "background radiation" include radioactive isotopes present in the ground, in brick and stone, in the air, and even in the body itself. In addition, cosmic rays, which apparently come mainly from outer space, are always bombarding the earth, and they cannot easily be avoided. Man has thus evolved and reached his present state of development in the presence of a certain amount of radiation exposure.

More than 40 years ago, when $\mathrm{X}$ rays and radium were in general use only for medical and dental purposes, standards were established for protecting operators against the possible effects of radiation. Originally, these standards were applied to radiologists and others in related activities, but with the development of nuclear power and other nuclear energy programs in many countries, the standards were extended to cover the general population, as well as those who were occupationally concerned in such programs.

In setting the protection standards, it was realized that radiation exposure might possibly involve some degree of risk, but the benefits were such that the risk was regarded as being small in comparison. With this in mind, several groups of experts have studied the problems of radiation protection and have made recommendations which are considered to be conservative, that is to say, they provide a margin of safety. These recommendations, which are regularly reviewed and are revised as may be required, are based on a considerable body of data, including extensive experience of medical radiologists in the use of $X$ rays and radium, the effects of various 
accidental (occupational) exposures to radiation, and the results of laboratory experiments. Among other things, they provide practical guides to what may be regarded as "acceptable" exposures to radiation for the general public. It is important to bear in mind that these exposures are far below those at which any effects have ever been observed on man or animals.

\section{Radiation Units}

In order to describe the recommended guidelines and standards for protection, something must first be said about the units that are commonly used to express doses of radiation. The first radiation unit to be introduced was called the "roentgen," now represented by the symbol R. Strictly speaking, the roentgen unit applies only to X rays and gamma rays and is a measure of the effect of these radiations on the air through which they pass rather than on the body in which they may be absorbed. For this reason, the roentgen is said to be a unit of "exposure" to radiation.

It was realized in due course that the effects of radiation were not determined so much by the exposure but by the amounts actually absorbed by the body. This led to the concept of an "absorption dose," which is expressed in terms of a unit known as the "rad," an acronym for "radiation absorbed dose." The rad unit is applicable to other kinds of radiations, such as alpha and beta particles and neutrons, and not merely to $X$ rays and gamma rays.

The dose in rads indicates the amount of energy that is absorbed by (or deposited in) any material (e.g., the human body), when exposed to various radiations. It has been found, however, that the amount of energy deposited by radiation in the body is not always a complete measure of the biological effect. The absorbed dose, expressed in rads, must be multiplied by quality (or effectiveness) factors for the given radiations and this gives the "biological dose." It is the biological dose, now referred to as the "dose equivalent," that is the most meaningful expression of the radiation dosage because it provides an indication of the expected effect of the radiation on the body. The unit of the dose equivalent is the "rem"; this term arose from its original use as an acronym for "roentgen equivalent (in) man."

Although the important quantity is the dose equivalent in rems, it is still common for radiation doses to be stated in roentgens or 
rads, as these are the measured quantities. This does not matter greatly, since radiological safety in underground nuclear detonations is primarily concerned with radioisotopes that emit beta particles and gamma rays within a relatively narrow range of energies. For these radiations the exposure (in roentgens), the absorbed dose (in rads), and the dose equivalent (in rems) are roughly the same for the exposure of the whole (or critical parts) of the body. In this book, the term "radiation dose" or "dose" will generally imply the dose equivalent in rems.

\section{Radiation Guidelines}

The guides for limiting radiation doses for people who are exposed to radiation in their daily work as well as for the general public have been developed on the basis of recommendations made by bodies consisting of authorities on radiological safety. These bodies are the International Commission on Radiological Protection, which was inaugurated in 1928, and the National Council on Radiation Protection and Measurements, established in the United States in 1929. In addition, problems of radiation protection of the population at large are considered by the United Nations Scientific Committee on Effects of Atomic Radiation and by the Committee on Biological Effects of Atomic Radiation of the U.S. National Academy of Sciences-National Research Council.

In 1959, legislation was enacted which created the Federal Radiation Council (FRC), composed of six members of the President's cabinet and the Chairman of the U.S. Atomic Energy Commission, to provide a national policy on human exposure to radiation. In developing this policy, the FRC relied to a considerable extent on the information developed by the bodies referred to in the preceding paragraph. The Federal Radiation Council was abolished by the President in December 1970 as part of a general reorganization of federal environmental programs and its functions were assumed by the Environmental Protection Agency.

The recommendations, in the form of Radiation Protection Guides, made by the FRC have been formulated into standards and regulations by the U. S. Atomic Energy Commission (U.S. AEC). The FRC has stated that there can be different guides with different numerical values depending on the circumstances. The standards for radiation protection given in the accompanying table apply to the 
general population (i.e., those nonoccupationally exposed) for normal peacetime operations. The standards are the radiation doses in rems per year (exclusive of the contributions from natural background radiation and from radiations used for medical and dental purposes) which are considered to be well below those that might be expected to have any significant biological effect.*

Radiation Protection Standards for the General Population

\begin{tabular}{lcc}
\hline & \multicolumn{2}{c}{ Rems per year } \\
\cline { 2 - 3 } & Individuals & Average of a suitable sample \\
\hline Whole body & 0.5 & 0.17 \\
Thyroid gland & 1.5 & 0.5 \\
\hline
\end{tabular}

It is seen from the table that the radiation protection standards for exposed "individuals" are three times as large as for the average of "a suitable sample." The significance is that where individual exposures are known from actual measurements, the annual wholebody dose equivalent should not exceed 0.5 rem. However, where the individual doses are not known, the average whole-body dose to a suitable sample of the exposed population, which can be estimated from radioactivity levels in the air, food, water, etc., should be not more than 0.17 rem per capita per annum. $f$

One of the considerations behind this difference in the standards for an individual and for the average of a suitable sample of the exposed population is that, in a large population, the radiation exposures will inevitably vary over a range of values. It is reasonable to assume on statistical grounds that only a small fraction of the individuals in a population group are likely to receive more than three times the average exposure. Hence, starting with the maximum of 0.5 rem per annum for the individual, the standard for the average whole-body dose for a suitable sample of the exposed population for

*The standards are sometimes stated in millirems $(0.001$ rem) per year; the numbers would then be 1000 times as large as those in the table, but would have exactly the same meaning.

†A "suitable sample" may be regarded as a fairly large group of people living in a given area who derive their food and water from more or less the same sources. For exposure to iodine-131, the "suitable sample" would include only young children. 
normal peacetime operations was set at one-third of this, namely, 0.17 rem per year. Moreover, for the population sample the average accumulated dose (above background and medical exposures) over a period of 30 years would not exceed about 5 rems. This has been considered to be tolerable from the genetic standpoint.

There is a specific standard for the thyroid gland because a large part of any radioactive (or stable) iodine isotope that enters the body is rapidly taken up by this gland. Since the thyroid is a small organ, the concentration of radioiodines could become quite large, and the radiation dose could thus greatly exceed the whole-body dose. Because it is somewhat less sensitive to radiation than many other parts of the body, however, the standard for the thyroid gland was set at three times that for the whole body. This means that the effective dose to the thyroid should not exceed 1.5 rems per annum (for an individual), even though the whole-body dose is less than 0.5 rem per annum, as it may well be. The corresponding average dose for a suitable sample of the exposed population would then be one-third of $1.5 \mathrm{rems}$, that is, $0.5 \mathrm{rem}$ to the thyroid per annum.

The standards for occupationally exposed individuals are about ten times those for nonoccupationally exposed individuals as given in the table above. Standards for occupational exposures were developed by considering an adult worker population of limited size wherein exposures are carefully controlled and monitored. Exposure is not continuous and the most sensitive members of the population, namely children, are not involved.

There is an important matter that must be pointed out in connection with the radiation protection standards. The policy of the U.S. AEC (and its contractors) is not merely to prevent exposures from exceeding the appropriate standards, but to keep them as far below as practicable.

Some scientists have argued that the current standards for radiation protection of the general public are too high for exposure over many years. Although this opinion is not widely accepted, the FRC, prior to its abolition, initiated a comprehensive review of the situation with regard to radiation exposures. This review is being continued under the direction of the Environmental Protection Agency. If it appears necessary, appropriate changes will be made in the Radiation Protection Guides and in the U. S. AEC's protection standards. It may be noted that a report entitled "Basic Radiation Protection Criteria," issued early in 1971 by the National Council on 
Radiation Protection and Measurements (Report No. 39), contains recommendations that essentially substantiate the FRC guidelines.

\section{Radioactivity Concentration Guides}

Radiation exposure of the body (or parts of the body) can arise from radioactive sources, particularly emitters of gamma rays, outside the body (p.14). This is referred to as "external exposure." In some circumstances, radioactive substances might enter the body by the ingestion of food and water, by inhalation, and by absorption through the skin. There is then a possibility of an "internal exposure" to radiation. The exposure of the thyroid gland to the radiations from radioactive isotopes of iodine deposited in that organ is an example of internal exposure.

For internal exposure, the FRC proposed Radioactivity Concentration Guides, which are fundamentally equivalent to the Radiation Protection Guides for external exposure. The former have been converted into concentration guides and cited in U.S. AEC standards, expressed as activity per unit volume of specific isotopes in air and water. These concentration guides are such that the internal dose (in rems) resulting from the unrestricted use of air and water by radiation workers and by the general public would not exceed the applicable Radiation Protection Guides. One-third of the concentration guide for an individual is taken as the guide for a suitable sample of the exposed population. The concentration guide for water is also used for milk consumed by the public.

The unit of activity commonly used to express the concentration guides is the "curie." This represents the quantity of any radioactive isotope undergoing $3.7 \times 10^{10}$ nuclear transformations (or disintegrations) per second. Because the curie is too large a unit for the concentrations that are normally encountered in connection with underground nuclear explosions, a fractional unit, called the "picocurie," is commonly employed. This activity unit is a millionth of a millionth part $\left(10^{-12}\right)$ of a curie, and represents a decay rate of 2.2 nuclear disintegrations per minute (i.e., one nucleus decays every 27 seconds, on the average).

Two radioisotopes of importance as potential internal radiation hazards are iodine-131, which could enter the body and be concentrated in the thyroid mainly as a result of the use of milk (e.g., by young children), and tritium, the radioactive isotope of 
hydrogen, which is most likely to be present in drinking water and other fluids. For iodine-131 in milk, the concentration guide is 0.1 picocurie per milliliter, based on the assumption that the intake of milk is about 1000 milliliters, that is, 1 liter ( 1.1 quarts) per day. The comparable concentration guide for tritium, consistent with the FRC recommendations, is 1000 picocuries per milliliter in water for regular consumption. Clearly, in equal concentrations, tritium is much less important than iodine-131 as a source of internal exposure to radiation. More will be said about tritium later (p.163).

\section{Background Radiation}

It is important that the FRC Radiation Protection Guides and U.S. AEC standards be placed in some sort of perspective. The natural background radiation, to which everyone on earth is exposed and cannot avoid, arises from cosmic rays, from radioactive uranium and thorium and their decay products and potassium-40 in the ground and in building materials, from radioactive gases in the air resulting from the decay of uranium and thorium, and finally (about 250,000 picocuries) from potassium-40, carbon-14, and other radioisotopes in the body. A rough breakdown, showing the average whole-body doses contributed at sea level by each important source for an adult, is given in the accompanying table.

Some people in the United States receive less natural background radiation than that given in the table, although rarely less than $0.09 \mathrm{rem}$, whereas others receive more but usually not more than

Average Whole-Body Doses from Natural

Background Sources at Sea Level

\begin{tabular}{|c|c|}
\hline Sources & Rems per year \\
\hline \multicolumn{2}{|l|}{ External } \\
\hline Cosmic rays & 0.030 \\
\hline $\begin{array}{l}\text { Uranium, thorium, potassium- } 40 \text {, } \\
\text { etc., in soil and building materials }\end{array}$ & 0.050 \\
\hline \multicolumn{2}{|l|}{ Internal } \\
\hline Potassium-40 in the body & 0.020 \\
\hline Carbon-14 and other isotopes & 0.005 \\
\hline Total & 0.105 \\
\hline
\end{tabular}


0.20 rem per year. People living in wooden houses at sea level have the lowest exposures, whereas those living in stone, brick, or adobe houses at high altitudes experience the largest doses. The latter building materials contain more naturally occurring radioactive substances than does wood, and at high altitudes cosmic rays are more intense than at sea level (e.g., 0.070 rem per annum at 5000 feet). The cosmic-ray intensity also increases with latitude from the equator to $50^{\circ}(\mathrm{N}$ or S) latitude.

In addition to the natural background radiation, many people receive radiation doses over limited parts of the body from medical and dental diagnosis and therapy. The dose equivalents from such exposures vary over a wide range, but an estimate of the average for the people of the United States, based on data published by the U.S. Public Health Service, is the equivalent of approximately $0.07 \mathrm{rem}$ per annum for the whole body.

It is of interest to mention that in certain parts of Brazil and India the background radiation levels are from 1.5 to 5 rems per year because of the large amount of radioactive thorium in the soil. Limited studies of the population in a high-thorium area of Brazil have so far yielded inconclusive results concerning the possible harmful consequences of radiation doses in excess of 1 rem per year.

\section{Important Radioisotopes in Underground Tests}

As far as potential radiation exposure is concerned, the important (or critical) radioisotope will depend on the circumstances. If there has been an accidental release of radioactivity from what was intended to be a contained underground detonation, the main radioisotopes in the air will be those of iodine and of the noble (i.e., almost chemically inert) gases krypton and xenon, all of which are fission products (p. 51). Radioactive isotopes of the same three elements may be released in very small amounts as a result of drilling operations in the chimney zone immediately after an underground detonation.

If natural gas, liberated by an explosion designed for the purpose of stimulating gas flow, is flared (i.e., burned) some months after the explosion ( $\mathrm{p}, 140$ ), the principal radioisotopes remaining are tritium and krypton-85. Because of their short half-lives, the isotopes of xenon and iodine will have decayed in the ground to negligible proportions. At the time of flaring, only a fraction ( 5 to 20 percent) 
of the tritium left by the explosion is found in the gas, most of the remainder being trapped as tritiated water (HTO). In the gas, the tritium is present mainly as tritiated methane $\left(\mathrm{CH}_{3} \mathrm{~T}\right)$ with a small percentage of tritiated hydrogen (HT). When the gas is burned in the air, the tritiated methane and hydrogen form nonradioactive carbon dioxide (and some carbon monoxide) and tritiated water. Hence, tritium, in the form of tritiated water vapor, and krypton gas are the significant isotopes that can enter the atmosphere when the natural gas is burned. Some of the tritiated water may subsequently be deposited on the surface, but the krypton- 85 remains in the air and its concentration is gradually decreased as a result of dispersion.

The most important substances for the radiological aspects of underground nuclear detonations, as far as entry into the atmosphere is concerned, are thus the radioisotopes of iodine, xenon, krypton, and tritium. These will be considered in turn. As noted earlier, radioiodine is primarily a problem as a potential internal hazard. Of the radioactive isotopes of this element, iodine-131 is normally the critical one. The most important biological pathway for iodine intake into the body is through the ingestion of cows' milk. For this situation, a concentration guide has been established (p. 161).

Both krypton and xenon are unreactive gases, chemically and biologically, and their solubility in body fluids is small. They can enter the body chiefly by inhalation, thereby leading to a certain amount of internal exposure to radiation. But this is very small and nearly all the radiation exposure from xenon and krypton would be external exposure arising from immersion of the body in the gaseous effluent in the atmosphere.

Tritium is negligible as a source of external radiation and only the possible internal exposure from this radioisotope needs to be considered. Tritiated water can enter the body by the ingestion of food (including milk) and water, by inhalation, or by absorption through the skin. In a short time the tritium becomes distributed throughout the water in the body. This isotope does not tend to concentrate significantly in any part of the body, as does iodine in the thyroid gland. For this reason and also because tritium emits beta particles of low energy and no gamma rays, it is regarded as one of the least potentially harmful radioisotopes.

The radioactive half-life of tritium is a little over 12 years, but the effective half-life in the body is usually about 12 days, on the average, because of the rapid elimination of water in normal 
metabolism.* That is to say, if a certain amount of tritium enters the body, it will decrease to half by the end of 12 days, to one-fourth after 24 days, and so on. As a consequence of the relatively short effective half-life, the amount of tritium in the body water does not accumulate and increase indefinitely even as the result of continuous consumption. Hence, the concentration guide for tritium in water (and other fluids) for unrestricted use has been set at 1000 picocuries per milliliter.

\section{Possible Radioactivity in Consumer Products}

As applied to public safety considerations, the radiation protection standards and concentration guides refer to external and internal exposures that may arise from radioactive materials that find their way into the air, into water, or into food supplies. With the development of certain aspects of the Plowshare program for peaceful applications of nuclear explosions, there is a potential source of radiation exposure in consumer products that may be used in the home or by industry.

The Gasbuggy (p. 48) and Rulison (p. 68) experiments, for example, were made to determine if underground nuclear detonations could stimulate the flow of natural gas in "tight" formations by breaking up deep underground rock. If these experiments continue to show promise, more complex experiments of a similar nature will probably be conducted. The initial gas released has contained small amounts of radioactive tritium and krypton- 85 which will be reduced still further by the development of appropriately "clean" nuclear explosives. But before any gas from a stimulation project is distributed commercially, regulations will be promulgated for the control of radioactivity in the gas reaching the consumer.

\section{RADIOLOGICAL SAFETY PROGRAM AROUND THE NEVADA TEST SITE}

\section{Introduction}

The U.S. AEC's radiological safety program off the immediate Nevada Test Site is conducted mainly by the Environmental

*In some tissues the effective half-life is longer, but these tissues would contain only a small proportion of the tritium present in the body. 
Protection Agency (formerly the U.S. Public Health Service) through the Southwestern Radiological Health Laboratory, Las Vegas, Nevada.* Support for the program is provided by the Air Resources Laboratory, Las Vegas, of the National Oceanic and Atmospheric Administration (formerly Environmental Science Services Administration), which supplies meteorological data and predictions of the expected trajectory and dispersion of possible radioactive effluent (see Chapter 7 ).

\section{Aerial Tracking and Sampling}

The chief objectives of aerial tracking are, first, to confirm if there has been an accidental release of radioactive residues from an underground explosion that was designed to be contained. And second, if such a release has occurred, the trajectory of the effluent is established so that radiation monitors on the ground may be informed about the areas where measurable exposures might occur.

Prior to each detonation at the Nevada Test Site, one or more airplanes carrying radiation detection instruments fly over and circle around the test location. The crews maintain contact by radio with the Control Point for the operation and with radiation monitors on the ground. After the detonation, the aircraft make low passes immediately over the explosion point to confirm whether or not there has been any detectable escape of radioactivity. The first indications would generally come from the RAMS array on the ground, described below.

If a release has occurred, the boundaries of the effluent are defined by utilizing sensitive instruments on board the aircraft, and the movement of the material is tracked until the radioactivity is no longer detectable. The information about the rate and direction of movement is used to determine where and when radioactive effluent may be expected to reach the ground off the Test Site. Radiation monitors can then take up positions at such locations prior to the arrival of the radioactivity.

At a later stage, an aircraft may be required to fly through the effluent so as to determine the peak radiation intensities at various distances from the test location. The data are then used, in conjunction with ground observations, to indicate the amount of

\footnotetext{
*Radiological safety support on the Test Site is provided by another contractor.
} 
radioactivity released to the atmosphere and the potential environmental contamination.

As a general rule, two aircraft follow any radioactive emission from an underground nuclear detonation. One is mainly occupied with tracking, whereas personnel on the second aircraft collect samples of the radioactive material. Some of these samples are tested immediately by instruments on board (Fig. 8.1), and others are taken to the Southwestern Radiological Health Laboratory for more detailed analysis.

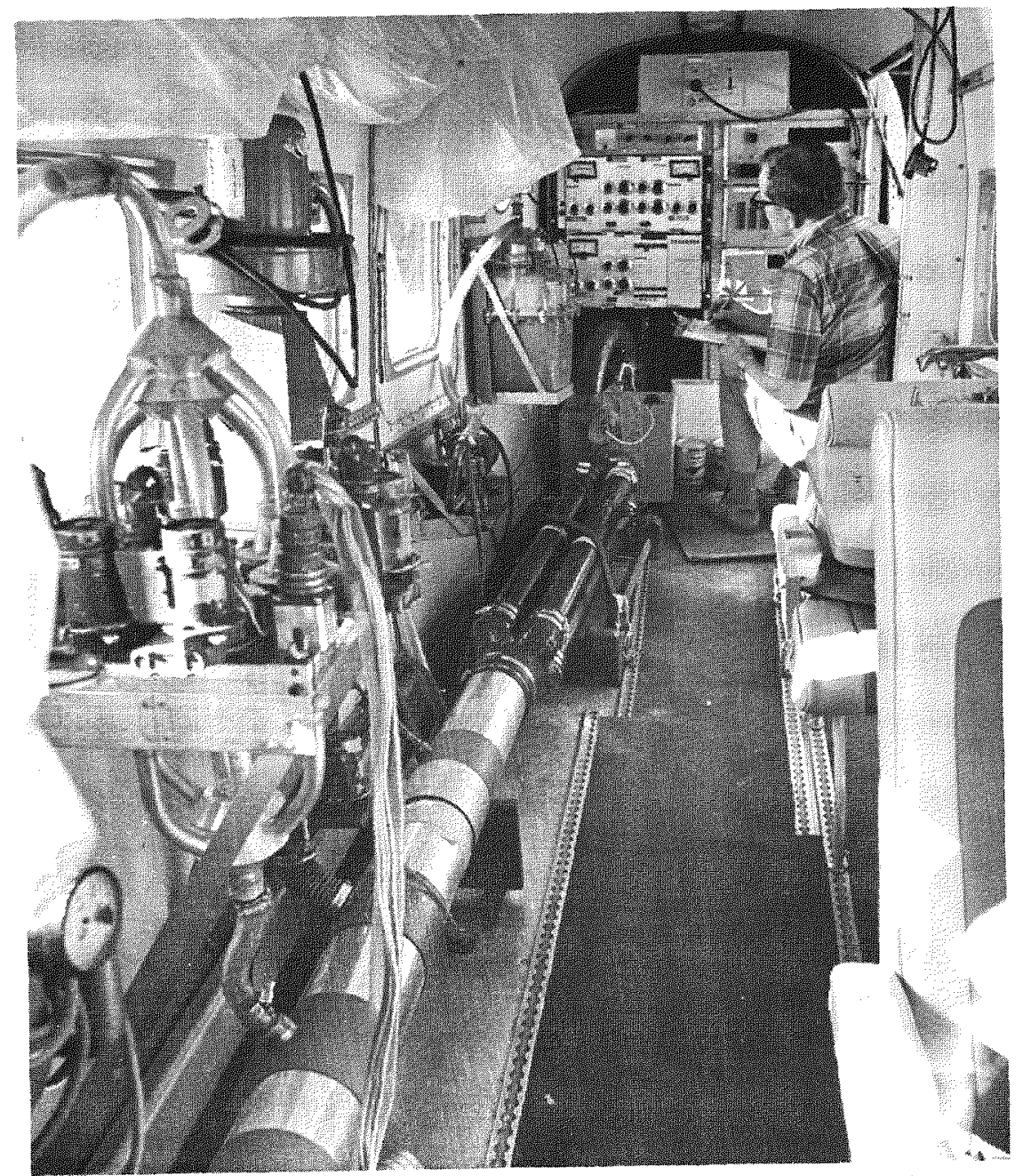

Fig. 8.1 Measuring instruments and sampling equipment in a Southwestern Radiological Health Laboratory aircraft. 


\section{Radiation Monitoring on the Ground}

If there has been a release of radioactivity to the atmosphere, the first indications of the occurrence, the extent of the release, and the direction in which the material is moving are usually obtained from the RAMS, an acronym for $R$ emote $A$ rea $M$ onitoring $S y$ stem.* This consists of arrays of permanent and temporary instrument stations for monitoring gamma-ray exposure rates on the ground within the Nevada Test Site. The temporary stations are set up for each event at fairly close-in points surrounding the particular explosion area. The instrument readings are recorded automatically at the Control Point for the event. Thirty permanent stations, which operate continuously, are distributed over the Test Site; if the radiation exposure rate at any station exceeds a preset level an alarm is sounded and appropriate action is taken. $\dagger$

For maintaining a continuous record of total radiation exposures in the area around (but outside) the Nevada Test Site, a network of almost a hundred permanent monitoring stations has been established (Fig. 8.2). At each of these stations there are instruments, called "dosimeters," which measure the total (external) exposure to gamma rays accumulated over a period of time. Two types of dosimeters have been used, namely, radiation-sensitive film badges, generally referred to as "film badges," and thermoluminescent dosimeters (or TLD's). Currently, only TLD's are being employed chiefly because they will record lower gamma-ray exposures than film badges.

The film badges were developed, like ordinary photographic film, and the degree of blackening was a measure of the exposure to gamma rays. The TLD's contain certain chemicals which become luminescent (i.e., they emit light) when heated after absorbing

*The RAMS is operated for the U. S. AEC by the on-site radiological support contractor.

$\dagger$ The radiation intensity or exposure rate is the amount of radiation to which an individual (or an instrument) would be exposed per unit time (e.g., per hour). The total (or accumulated) radiation exposure is the amount of radiation that would be received over a period of time (e.g., many days). Generally, different instruments are used to measure exposure rates and total exposures. However, total exposures can be computed (by integration) if the exposure rates are known over the exposure period. Exposure rate may be compared to the speed of an automobile (in miles per hour) and total exposure to the distance covered (in miles). 


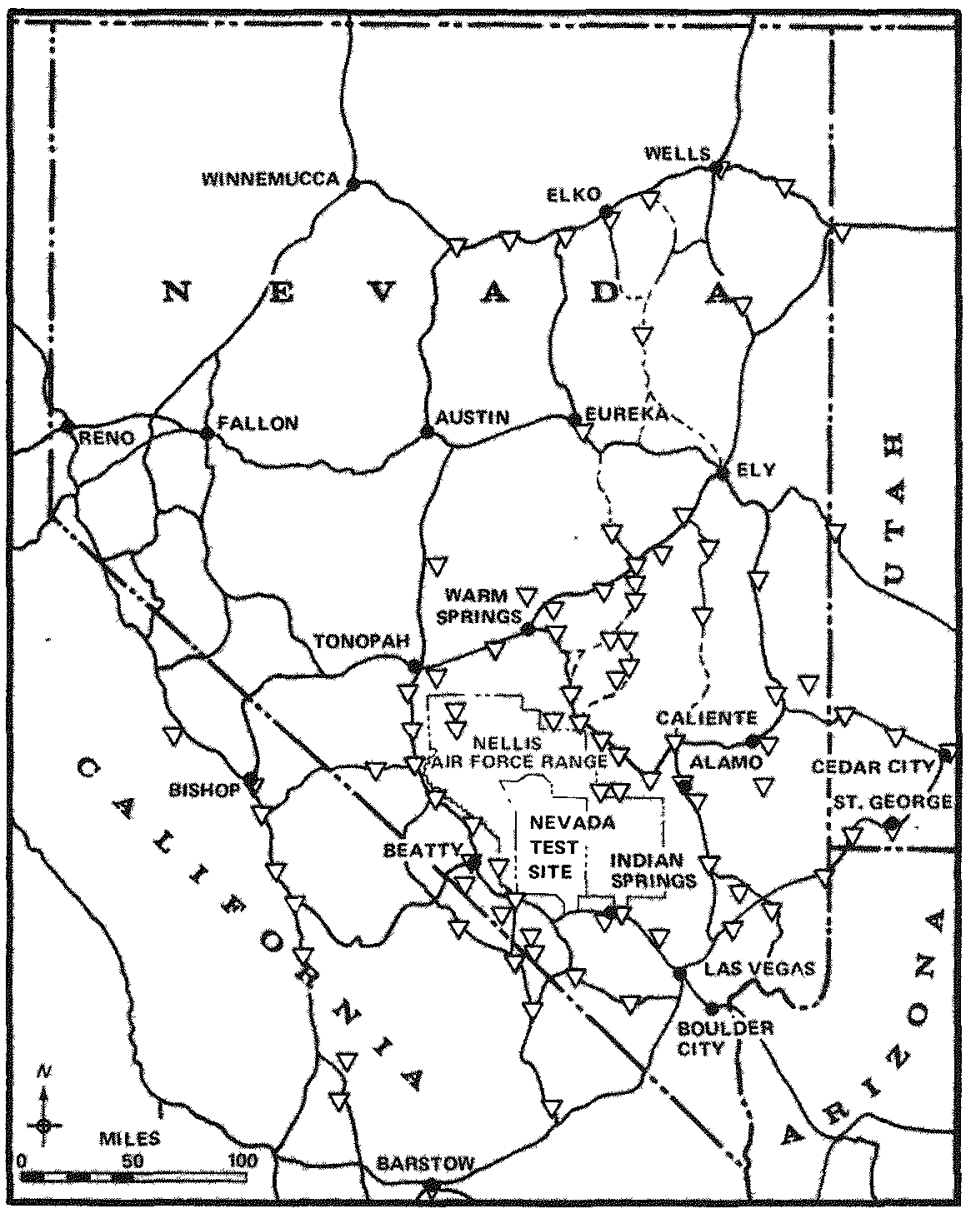

Fig. 8.2 Radiation dosimeter stations $(\nabla)$ around the Nevada Test Site.

radiation. The intensity of the light is related directly to the radiation exposure.

In normal circumstances, the dosimeters are collected (and replaced) monthly and taken to the Southwestern Radiological Health Laboratory to be read. If there is a release of radioactivity following an underground detonation, the dosimeters are read at shorter intervals. The readings indicate the amount of external exposure to gamma radiation that would have been received by people living in the vicinity of the various stations during the intervening period. Since the stations operate on a routine basis, the 
instruments provide a continuing record of external radiation exposures both before and after an underground nuclear detonation.

Furthermore, a few permanent residents living in the vicinity of each of the routine monitoring stations within a 200-mile radius of the Nevada Test Site wear dosimeters. These are usually collected for reading at monthly intervals and are replaced by fresh ones. In this manner, the actual radiation exposures of individual persons, as well as in the general area, can be estimated.

In order to obtain a more rapid indication of any changes that may occur in the radiation situation, suitable instruments are located at about one in four of the stations referred to above. These instruments provide a continuous record on charts of the local exposure rate. The charts are changed daily if the radiation levels are significantly above the normal background (Fig. 8.3).

In addition to routine monitoring, exposure rates and total exposure are measured after every underground nuclear detonation with both mobile and stationary instruments. The number of mobile monitoring vehicles deployed in the field depends on the type of event, and their positions are determined by the trajectory of the radioactive material. Each mobile monitoring unit is operated by trained personnel who can make measurements and report the results by means of a two-way radio. The vehicles carry instruments for indicating radiation exposure rates as well as equipment for collecting samples of air, water, milk, etc. (Fig. 8.4).

The data compiled by the mobile monitors are supplemented by a number of portable exposure-rate meters placed at suitable locations where they operate unattended. They provide a continuous record of radiation intensities immediately after the detonation. The total external exposure in the first few days is determined from TLD's placed on roads in a number of arcs across the predicted downwind fallout sector (p. 236). If necessary, the accumulated exposure can be estimated by integration (p. 47) of the exposure-rate meter records and from field monitoring results.

\section{Off-Site Environmental Surveillance for the Nevada Test Site}

The off-site environmental surveillance aspect of the radiological safety program for the Nevada Test Site includes networks for the collection and analysis of samples of air, water, and milk. Analysis is done regularly on a routine basis, but more frequently after an 


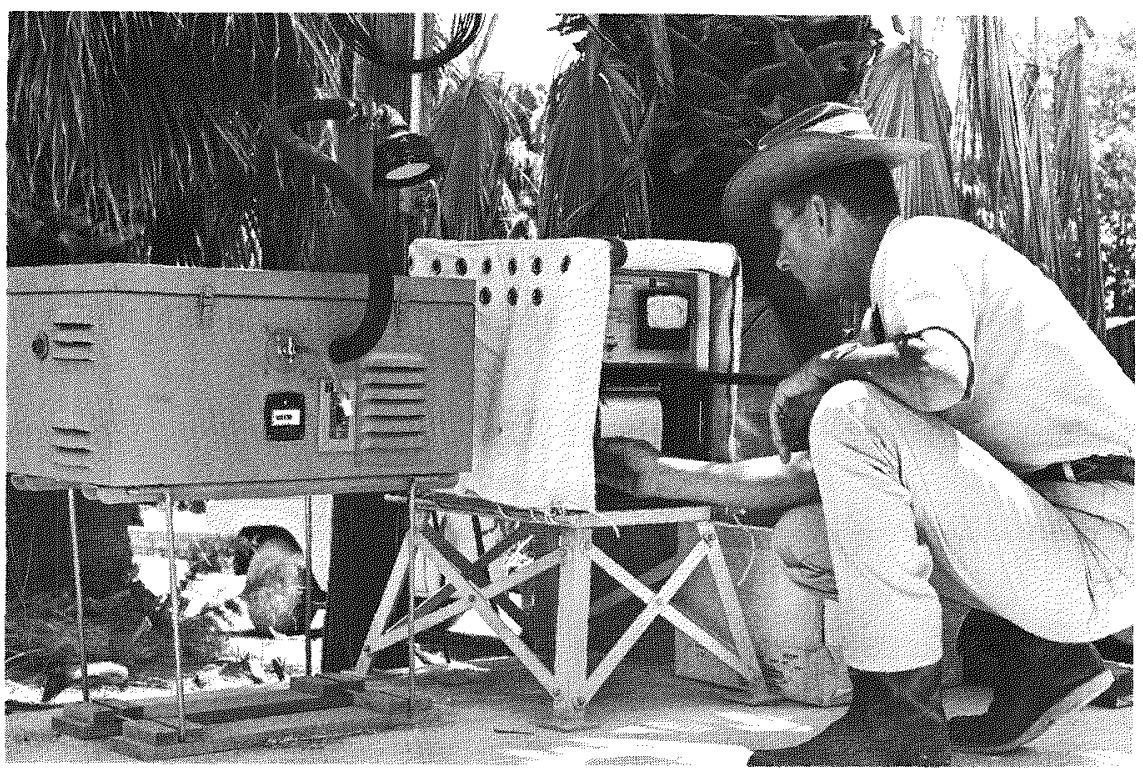

Fig. 8.3 Continuous radiation exposure rate recorder with strip chart (right) and an air sampler (left) at a ranch in Nevada.

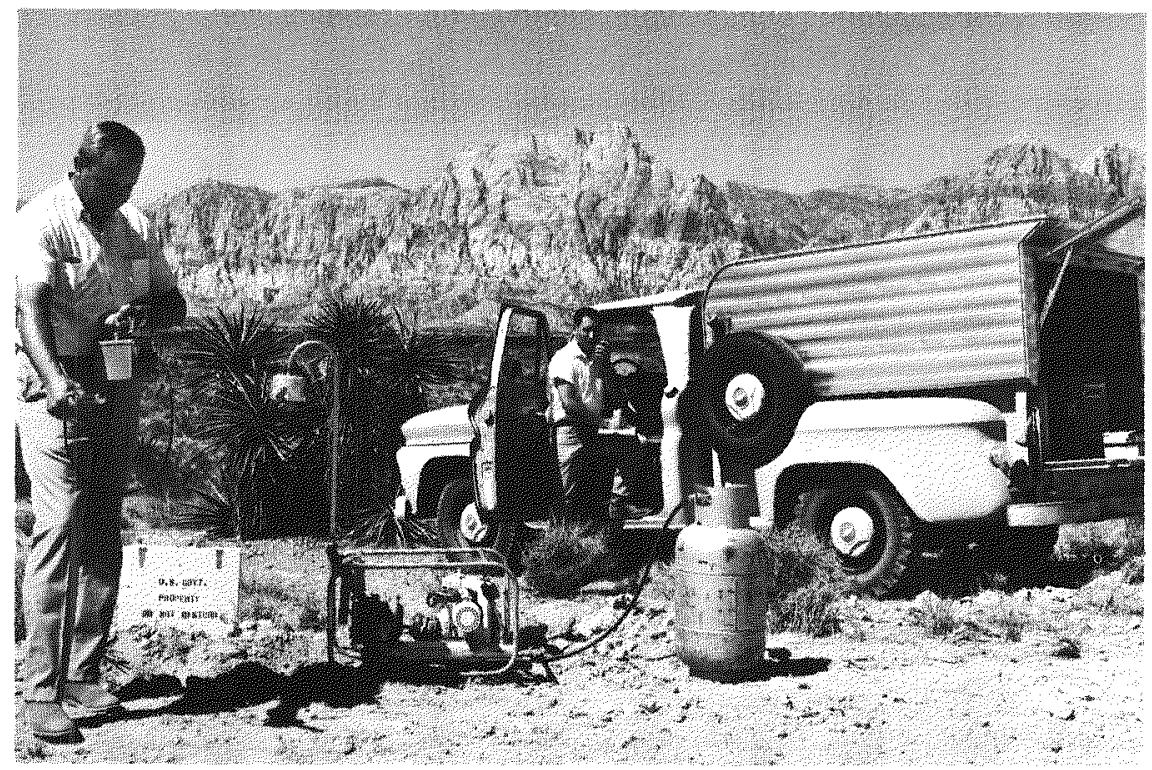

Fig. 8.4 Mobile monitoring station in a desert valley in Nevada. (Bottled gas is used to drive the motor for an air sampler.) 
underground detonation which may have been accompanied by the release of radioactivity to the atmosphere.

There are almost a hundred permanent air sampling stations (see Fig. 8.3) and ten standby stations, with at least one in nearly every state west of the Mississippi River. In most cases the sampler contains only a filter for removing particulate matter from the air. The stations are operated by private citizens who mail the filters daily to the Southwestern Radiological Health Laboratory where they are tested for beta-particle activity. If this activity is high enough to warrant it, the samples are also analyzed for gamma-ray emitters. In some twenty of the stations, the sampler includes an activated charcoal cartridge for absorbing iodine and other elements. The gamma-ray emission from the cartridge is analyzed to identify the radioactive isotopes present and to determine their amounts. Cartridges are also available at the stations where they are not used regularly, and they can be inserted by the operator upon notification by telephone.

If the observations made from aircraft and by ground monitors indicate that radioactive matter is passing over a particular area, mobile air samplers, similar to those used in the routine network, are put into operation. Normally, the exposed filters are taken immediately to the laboratory and are tested to determine the affected area and the general concentration of radioactive effluents at ground level. From the latter, the potential hazards from inhalation and from ingestion, especially of radioiodine in milk, are evaluated.

In the search for radioisotopes that might have originated from nuclear explosions, water samples, from both surface and subsurface sources, are collected on a monthly basis from stations in Nevada, California, and Utah (Fig. 8.5). They are tested for total radioactivity and for specific radioisotopes. Selected samples are also analyzed for radium, uranium, and tritium, which are all naturally-occurring radioactive substances.

Some forty milk producers within a 250 -mile radius around the Nevada Test Site (Fig. 8.6) cooperate in the milk sampling program. The sources range from family milk cows to grade-A dairies. Samples are collected monthly and are analyzed for various radioactive isotopes. In addition, commercial (processed) milk samples for analysis are obtained weekly from 63 major cities throughout the United States from the Pasteurized Milk Network of the Environmental Protection Agency. 


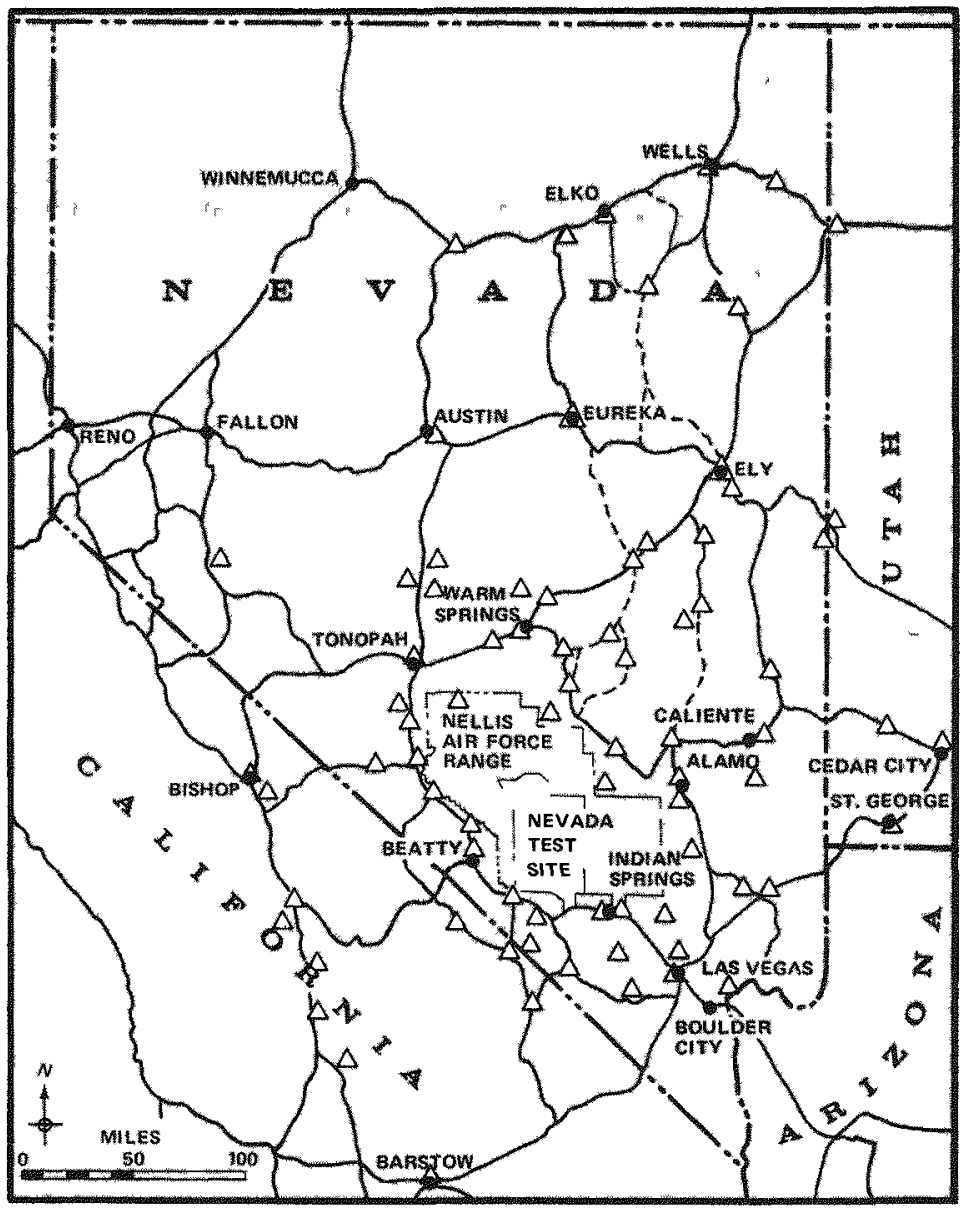

Fig. 8.5 Water sampling stations $(\Delta)$ around the Nevada Test Site.

After an underground nuclear detonation in which there has been a release of radioactivity to the atmosphere, milk samples are taken more frequently in the area over which the radioactivity is known to have passed. Should there be any reason to suspect that radioactive material has been transported a considerable distance from the Nevada Test Site, milk samples can also be obtained at short notice from a standby network of 150 grade-A milk processing plants in eleven western states.

The sampling locations are changed from time to time, as may be indicated by the results of the analyses. In selecting these locations, 


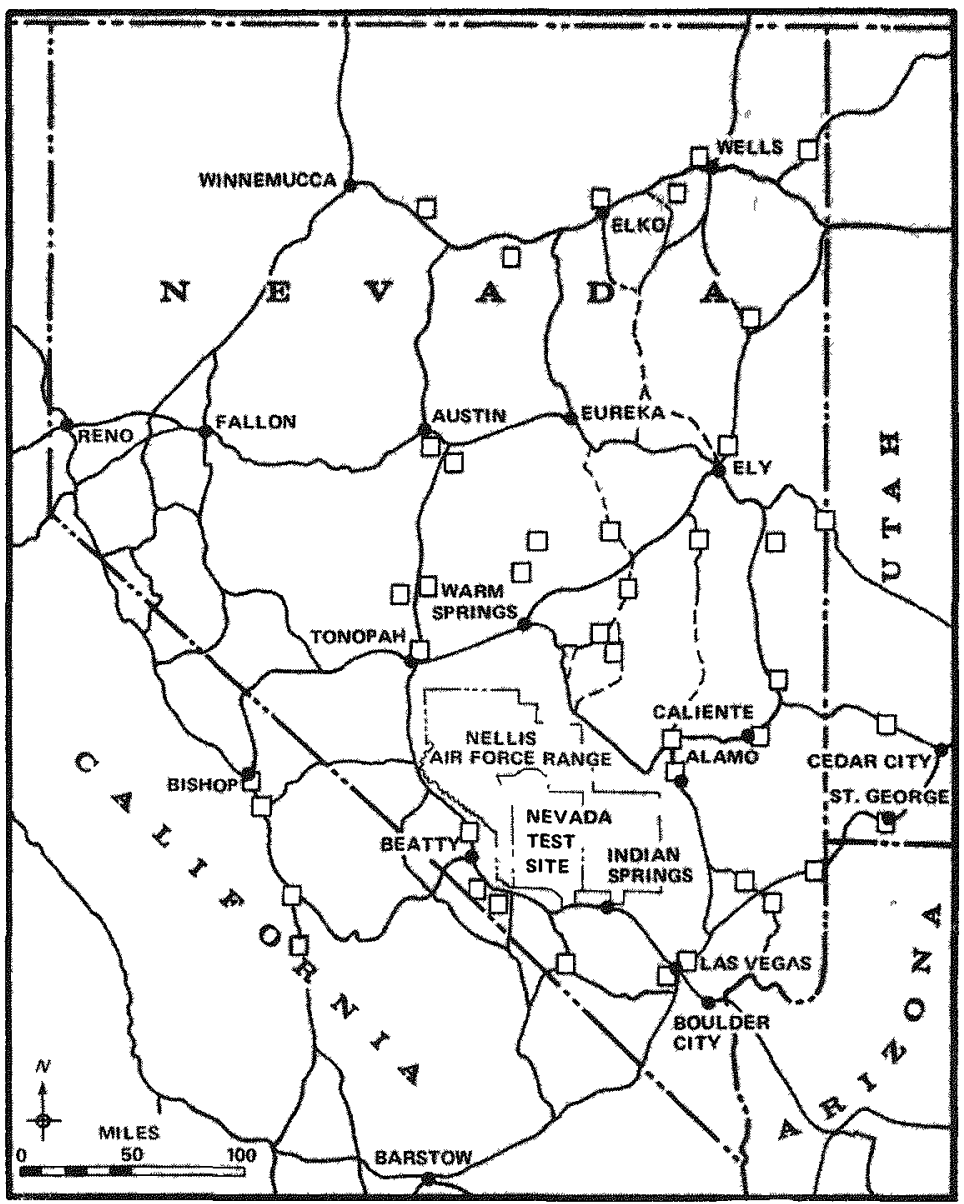

Fig. 8.6 Milk sampling stations ( $\square$ ) around the Nevada Test Site.

the fact is kept in mind that the peak concentration of iodine-131 in milk is not reached for 2 to 5 days if the cow's intake was by ingestion of contaminated feed. If the iodine-131 is present as a result of inhalation, then the peak is reached after about a day.

In order to assess the consequences of a particular release of radioactivity, samples from some special cities in the Pasteurized Milk Network may be obtained daily instead of weekly. Analysis of milk samples from this network, as well as from the standby network referred to above, would be continued for a week or so until the iodine-131 concentration reached normal background levels. 
If there is any significant release of radioactivity from an underground detonation, samples of growing vegetation and of cows' fodder are collected in the downwind direction and analyzed. The results are used to determine the area and centerline of cloud passage and also the expected concentrations of radioactive isotopes in milk.

One of the objectives of the surveillance program is to maintain a constant awareness of the levels of radioactivity in various materials that may be consumed by people and animals. If the surveillance should indicate that protective measures for the general public are desirable, appropriate actions could be taken immediately. Some of these are described later in this chapter.

\section{Measurement of Internal Exposure}

If an underground nuclear detonation at the Nevada Test Site has resulted in the release of radioactive materials to the atmosphere, measurements can be made of radioisotopes, such as tritium and iodine-131, that may have entered the human body either by ingestion of food and drink or by inhalation. The crews of the tracking aircraft and members of ground monitoring teams are assayed as necessary for the presence of internal radioactive material.

An instrument called a "whole-body counter" is used to measure the amounts of individual gamma-ray emitting isotopes in the body. A form of the same type of instrument, referred to as a "scanner," can detect and measure these isotopes in specific parts of the body. For example, the amount of iodine-131 in the thyroid gland can be determined in this manner. Stationary whole-body counters and scanners are located at the Southwestern Radiological Health Laboratory. A mobile scanner is also available for use in the field.

Since tritium does not emit gamma rays and the beta particles have low energies (and short ranges), it cannot be measured by the whole-body counters and scanners. Consequently, analyses are made in the laboratory of samples of urine. From the rate of excretion of tritium, the amount of this radioactive isotope in the body and the dose equivalent at any time can be estimated.

The foregoing techniques for determining levels of internal radiation are used for those who are occupationally exposed and also for off-site residents when it appears to be desirable. 


\section{RADIOLOGICAL SAFETY FOR DETONATIONS OFF THE NEVADA TEST SITE}

\section{Introduction}

Detonations off the Nevada Test Site fall into two main categories: those which are related in some manner to the weapons program, atthough not necessarily actual tests of weapons, and those which are part of the Plowshare program. For all such detonations, a radiological monitoring program is established, usually by the Southwestern Radiological Health Laboratory, and a network may be set up for sampling air, water, and milk, in particular. The general procedures have been similar to those employed in the vicinity of the Nevada Test Site, but modified to suit the particular location and type of detonation. The Air Resources Laboratory also provides the appropriate meteorological support services as in Nevada (p. 165). Since the radiological safety operation will vary with the circumstances, some of the following material is written in conditional form. However, a definite program is prepared and implemented for every operation.

\section{Radiation Monitoring}

For detonations outside the Nevada Test Site, the probability that radioactivity will be inadvertently released to the atmosphere is very small. Nevertheless, aircraft may fly over the explosion vicinity immediately after the detonation and the RAMS (p. 167) may be used to detect radioactive material that might escape into the atmosphere. Furthermore, an appropriate radiation monitoring network may be established on the ground in the offsite area before each event as the particular situation may require. The network might consist of fixed stations, usually at population centers and along existing roads and highways, and mobile units. The latter would carry continuous exposure-rate recorders so that significant radiation levels could be detected immediately. Dosimeters for recording total accumulated exposures to gamma radiation would be located at the fixed stations.

In a Plowshare gas-stimulation experiment, it would be necessary to drill back into the chimney some time after the event in order to evaluate the results. A radiation monitoring program would then be 
operated along the lines indicated above. Fixed and mobile stations may be supplemented by aerial surveys. If gas is to be flared, an environmental monitoring program may be conducted, if necessary, during the first few days with instrument stations and sampling systems in places indicated by the prevailing meteorological conditions. The program would be continued at an appropriate level as long as flaring is in progress.

The monitoring instruments referred to above serve mainly to detect and measure gamma-ray exposures. Although tritium and krypton- 85 are among the least hazardous of all radioactive species, they are the important isotopes in the flared natural gas. Tritium emits no gamma rays and krypton- 85 does so in only a small fraction of its disintegrations. Consequently, the amounts of these isotopes would be determined by taking samples of air and water for analysis, as described in the next section.

\section{Environmental Surveillance}

An important aspect of the environmental surveillance program off the Nevada Test Site is associated with detonations conducted to stimulate the flow of natural gas. To determine the radioactivity of the gas and its decrease with time, samples withdrawn from the rubble chimney are collected at regular intervals. They are analyzed for tritium and krypton-85, in particular, using procedures capable of detecting very small concentrations of these isotopes (Fig. 8.7).

To assure public safety, gas would be flared only if the analyses and meteorological conditions were satisfactory. As a result of turbulent mixing and of spreading by winds, the level of radioactivity in the surrounding air is very much less than in the flared gas. In any event flaring can be stopped instantly should it be desirable (p. 140). As indicated on page 164, nuclear explosives under development will produce even less tritium than those used in the past for gas stimulation experiments.

The radiological safety plans for drillback and flaring operations associated with the Rulison experiment embraced surveillance of air, food (including milk), and water supplies using samples collected at a network of local stations. More distant stations were available on a standby basis if required. Prior to and in the course of the operations, samples of food and water normally consumed by people, by domestic livestock, and by selected wildlife (e.g., deer) 


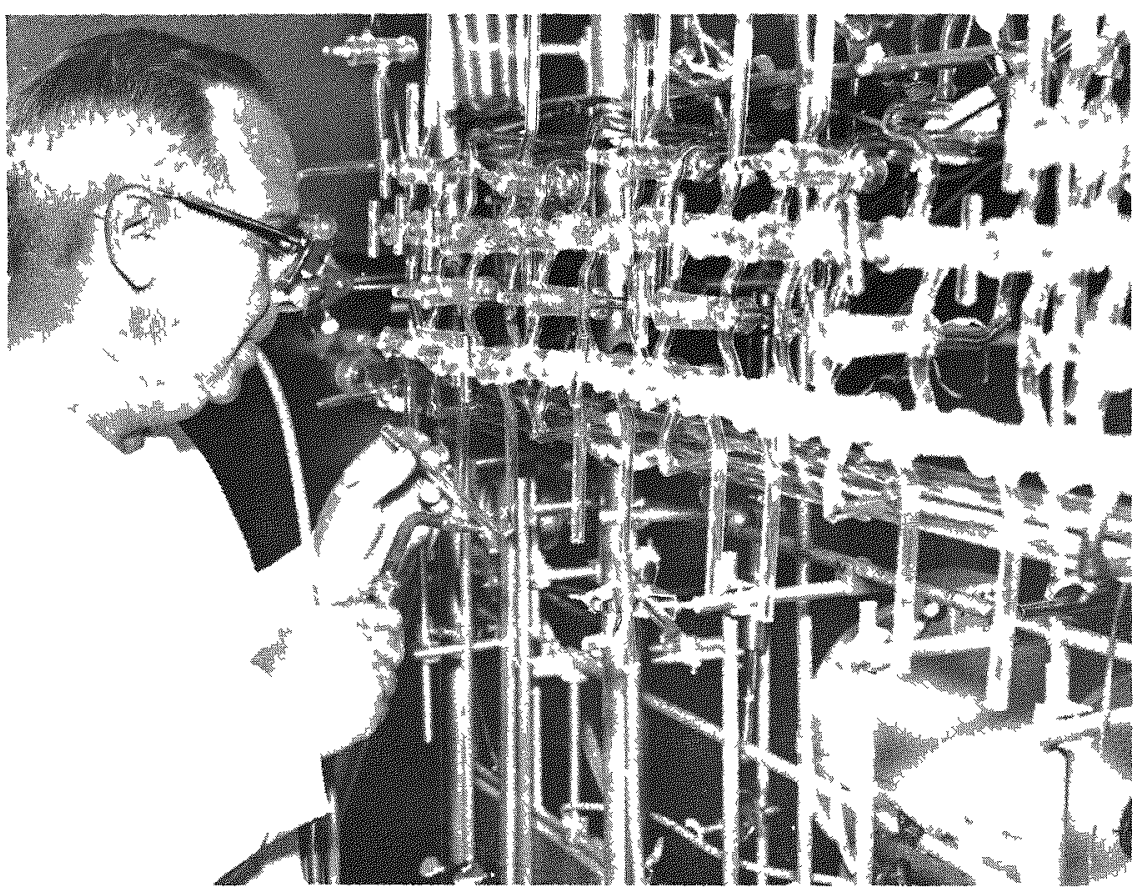

Fig. 8.7 Equipment at the Southwestern Radiological Health Laboratory for the separation of gases prior to analysis.

were collected. These and air samples were analyzed for tritium and other radioisotopes; only the air was tested for krypton- 85 since it is negligible as an internal hazard and the concentrations in food and water are always extremely small. Specimens of vegetation and soil and also of urine from the inhabitants were obtained for analysis from representative locations around the flaring site. Samples of wildlife in the area were taken prior to drillback and also during flaring, and selected organs were tested for fission products and tritium.

\section{PROTECTIVE STUDIES AND ACTIONS}

\section{Radioiodine Program}

Because of the critical importance of the uptake of radioactive isotopes of iodine by the thyroid gland, especially of young children, 


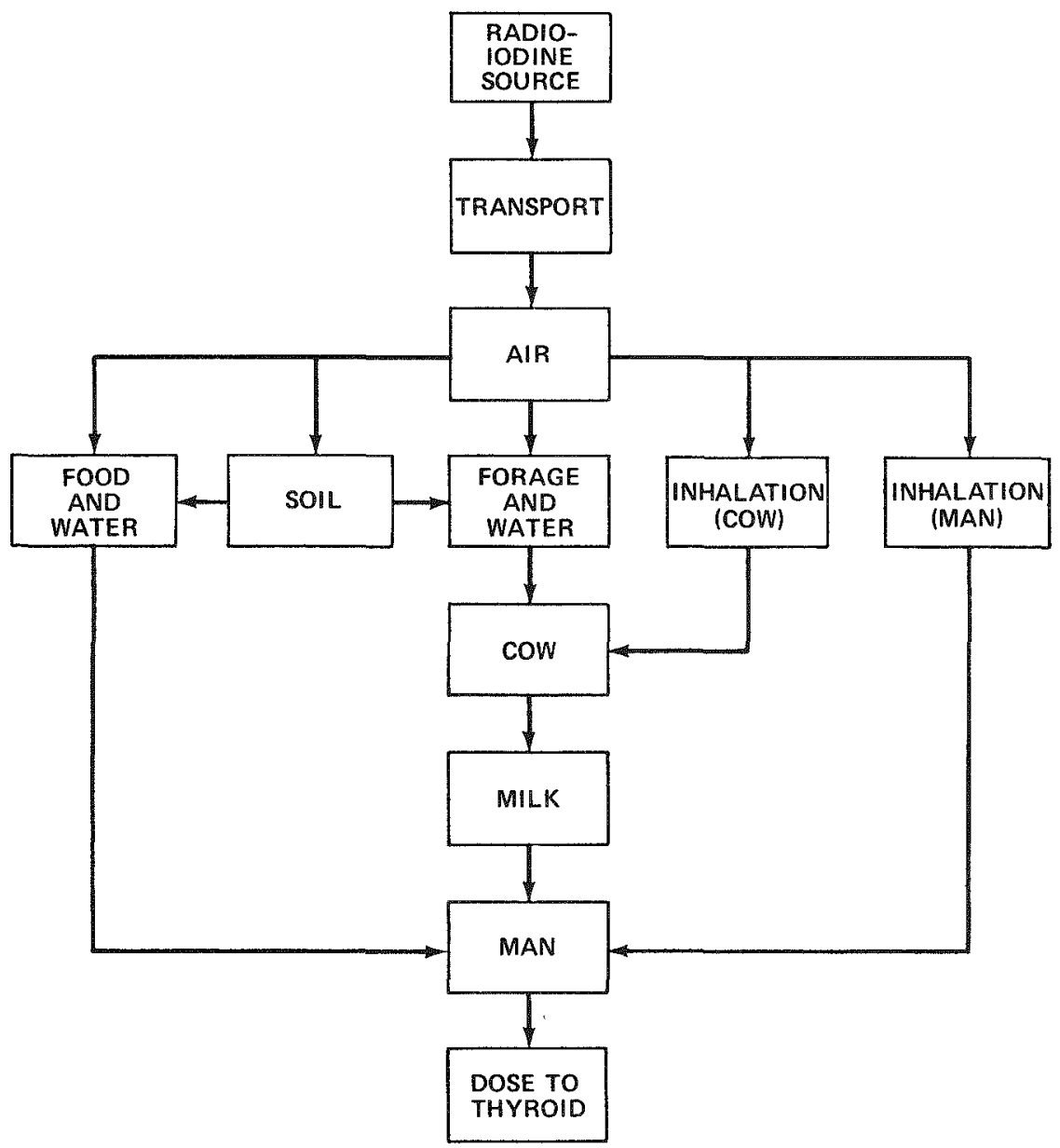

Fig. 8.8 Paths of radioiodines from fission products to man.

a program was established in 1963 by the U. S. Atomic Energy Commission, through the Southwestern Radiological Health Laboratory, to study the radioiodine problem. In the event of an accidental release of iodine to the atmosphere, the movement through the environment to man would be as depicted in Fig. 8.8.

Most of the radioactive iodine that enters the thyroid gland of children comes from cow's milk. Although the cow may obtain some radioiodine from the air by inhalation and from water, the main source is grass or other feed that has become contaminated directly with fallout. About 10 percent of the total intake of iodine, both radioactive and stable isotopes, appears in the cow's milk. The ratio 
of radioactive to nonradioactive iodine in the milk is essentially the same as in the feed.

As part of the radioiodine program for radiological safety in connection with underground nuclear detonations, field experiments are carried out for two types of situations. The first includes explosions, such as in experiments for earth excavation studies (see Appendix), in which the release of radioactivity to the atmosphere is, to a large extent, predictable. The second involves cases where radioactivity escapes accidentally.

Some of the important data obtained from the tests made on milk samples are the following: time after ingestion of contaminated forage for the radioiodine concentration in milk to reach a maximum; rate of decrease of the radioiodines in milk under various conditions after the peak has been reached; and the percentage of the radioactivity in the forage that subsequently appears in the milk.

When there is an accidental release of radioactivity at the Nevada Test Site, an attempt is made to obtain information similar to that described above. The studies are limited, however, to a few dairy farms in the immediate area where cooperation has been established in advance. An example of this type of situation is described in a later section.

To provide support for the radioiodine program, an experimental dairy farm has been operated by the Southwestern Radiological Health Laboratory at the Nevada Test Site for several years (F1g. 8.9). At this farm, alfalfa and other forage crops have been

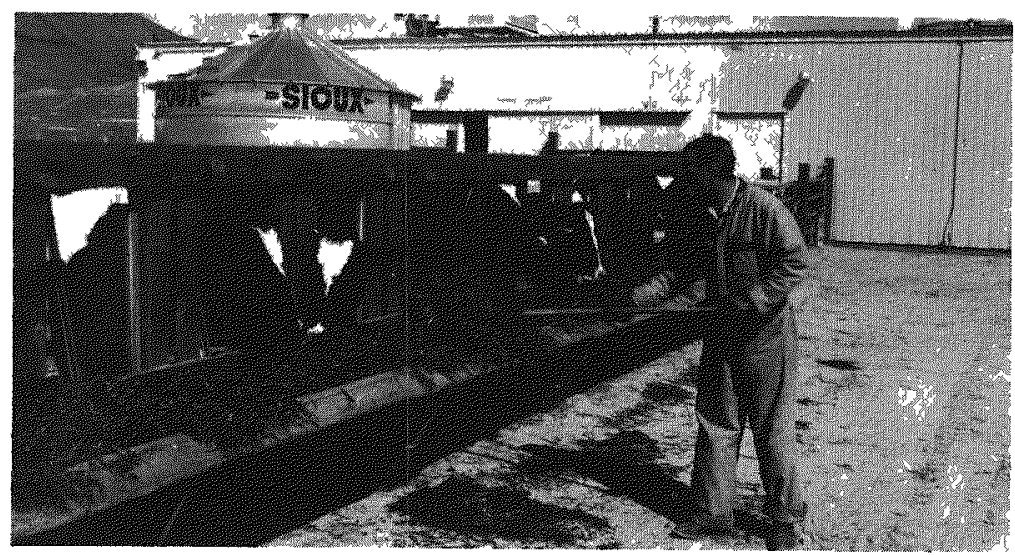

Fig. 8.9 Freshly cut green chop being fed to dairy cows at the Southwestern Radiological Health Laboratory's Experimental Farm on the Nevada Test Site. 
grown and exposed to radioiodine (and other fission products) by releasing known amounts over the plants. The crops have been harvested and fed to lactating cows. Both the forage and milk have been subjected to the same tests as the samples obtained from field stations. From the results of these experiments information is available for predicting the peak levels of radioiodine in milk from cows that consume forage contaminated by fission products.

\section{Other Radioisotopes in Milk}

Radioisotopes of iodine are of critical importance when the radioactive material escaping into the atmosphere consists largely of fission products. But for excavation experiments using explosive devices with relatively small yields of fission energy, other radioisotopes would probably be more significant biologically. A farm study program has, therefore, been initiated to include radioisotopes of other elements.

Dairy products, such as milk for children, are a major component of the food chain of man. Consequently, metabolism studies are being conducted to determine the fate of radioisotopes of several different elements, not present among the fission products, added to the feed of lactating cows. Experiments have been made with radioactive isotopes of the elements hydrogen (i.e., tritium), beryllium, lead, rubidium, thallium, and tungsten; additional studies are planned for iron, manganese, mercury, plutonium, and ruthenium.

\section{Protective Actions}

Appropriate measures have been developed for taking protective action in connection with an underground nuclear detonation to prevent any hazard to public health. These measures are examined in preparation for every test, and plans are made for dealing with any radiological situation that may arise, be it expected or highly improbable. The lack of a satisfactory plan for protective action would be sufficient grounds for canceling or delaying the event. If there is a possibility that any effluent will pass over an inhabited area while the radioactivity level is still fairly high, there must be assurance that the people can be evacuated temporarily or advised to take suitable cover before the effluent reaches the area. To facilitate any protective action that may be desirable, the Southwestern Radio- 
logical Health Laboratory obtains detailed information concerning the locations of people and of milk cows in the vicinity of the Nevada Test Site.

The protective actions outlined above apply to external exposure. Protective action is both feasible and effective against internal exposure, in particular for reducing the dose to the thyroid resulting from drinking milk contaminated with radioactive iodine. It is recognized that milk may not be the only significant source of radioiodines for adults, but it is of critical importance for young children. The protection of milk supplies is discussed in the next section.

\section{Protection of Milk Supplies}

If fission products should escape into the atmosphere, the major portion of the radioiodine appearing in cows' milk would be derived from eating contaminated forage, rather than from inhalation or open water supplies. A positive protective action would then be to substitute uncontaminated fodder for the contaminated forage as soon as possible. For cows that have eaten forage contaminated with fission products, the effective half-life of radioiodine-131 in their milk is about 5 days. But replacement of the forage by uncontaminated hay results in a reduction of the effective half-life to 1 to 2 days. In other words, the radioiodine in the milk decreases quite rapidly if the contaminated forage is replaced by uncontaminated feed.

The situation that arose after the accidental release of radioactivity from the Pin Stripe event at the Nevada Test Site in April 1966 is of interest in this connection. The highest radioactivity level of iodine-131, namely, 4.8 picocuries per milliliter of milk, was found at a dairy in Hiko, Nevada, 65 miles from the explosion point. The cows at this dairy had been feeding on fresh green chop that had become contaminated by radioactivity from the nuclear detonation.

Although the iodine-131 in the milk was much less than the Federal Radiation Council's Protective Action Guide, at which protective action is recommended in case of an emergency, the contaminated green chop was replaced by uncontaminated hay when the concentration of iodine-131 in the milk was at about its peak value. This was done as a precautionary measure to avoid any unnecessary exposure to radiation. Within a short time, the radio- 


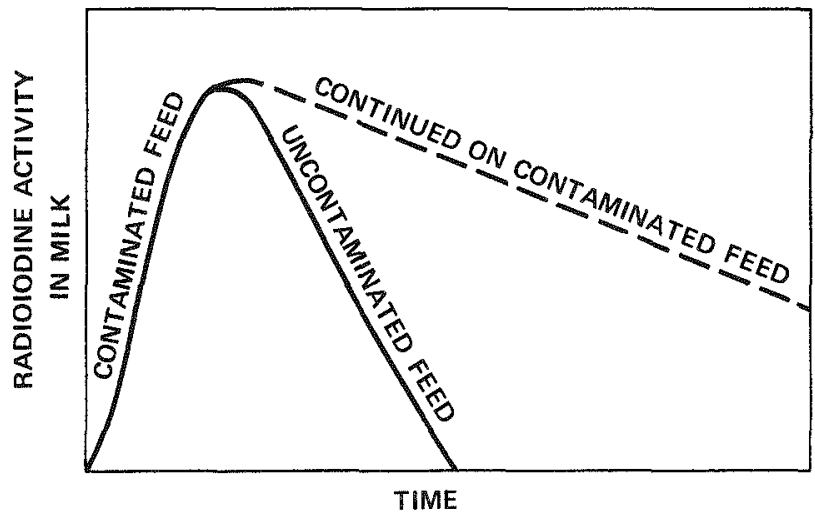

Fig. 8.10 Radioiodine in milk after cows' consumption of contaminated feed (not to scale).

activity of the milk began to drop sharply, as indicated in Fig. 8.10. Four days after starting the uncontaminated fodder, the radioactivity level had fallen to 0.14 picocurie per milliliter.

The full curve in Fig. 8.10 shows the variation with time of iodine-131 activity in the milk produced; the initial rapid increase, resulting from the consumption by the cows of contaminated green chop, was followed by a marked decrease when uncontaminated hay was substituted. The broken line indicates the radioactivity in milk from cows kept on the original contaminated forage. The potential dose equivalent of iodine-131 to the thyroid gland of a person consuming the milk was reduced an estimated 70 percent by using the uncontaminated fodder.

The milk from the dairy at which the test was made was picked up by tank truck, in the usual way, and transported to Las Vegas, Nevada, for processing. The peak radioactivity after dilution by other milk in the tank was 0.1 picocurie per milliliter. As the result of natural radioactive decay and normal operating procedures, no radioiodine was detectable in the processed milk sold in Las Vegas.

In the path of the fallout from the Pin Stripe test, there were a few ranches where cows were kept to provide milk for the families living there. This milk would not have been subjected to the dilution and processing that would have decreased the radioiodine activity. Consequently, all the milk from these family cows was collected as a prudent measure and used for experimental purposes. It was replaced by milk from uncontaminated sources. 
After the Pin Stripe event, 78 people living in the fallout area were monitored for the presence of radioiodines in the thyroid gland (p. 174). In 25 cases the amount was not detectable above the normal background. The estimated dose equivalent to the thyroid was less than 0.05 rem in 34 people and the remaining 19 had projected doses between 0.05 and $0.30 \mathrm{rem}$. These figures may be compared with the radiation protection standard value of 1.5 rems per annum for an individual, as given on page 158 .

Another way in which milk containing some radioiodine-131 could be treated would be to divert it to cheese making. Because of the relatively short half-life of this isotope (about 8 days), the radioactivity would be so small as to be undetectable above background when the cheese reached the market.

\section{MEDICAL AND VETERINARY INVESTIGATIONS}

\section{Medical Investigations}

The Medical Liaison Officer Network (MLON) has been established to investigate reported cases of possible radiation injury sustained by members of the general public as a result of nuclear detonations. Almost all of the 50 states are represented in the MLON which consists of physicians who have substantial knowledge and experience of radiation effects.

A MLON investigation is instituted upon receipt of an inquiry concerning the possibility of radiation injury resulting from the release of radioactivity from an underground nuclear explosion. Initial inquiries in the past have come from the Federal Bureau of Investigation, State Health Departments, and various regional operations offices of the U. S. Atomic Energy Commission. These inquiries are forwarded to the MLON Coordinator at the Southwestern Radiological Health Laboratory. The Coordinator reviews the inquiry as transmitted, and if indeed the reputed radioactivity may conceivably have resulted from a nuclear test, the appropriate MLON physician is assigned to investigate the case (Fig. 8.11).

The MLON physician makes contact with the patient and with the patient's physician in order to obtain firsthand information about the purported exposure and the resultant injury. The Southwestern Radiological Health Laboratory then provides environ- 


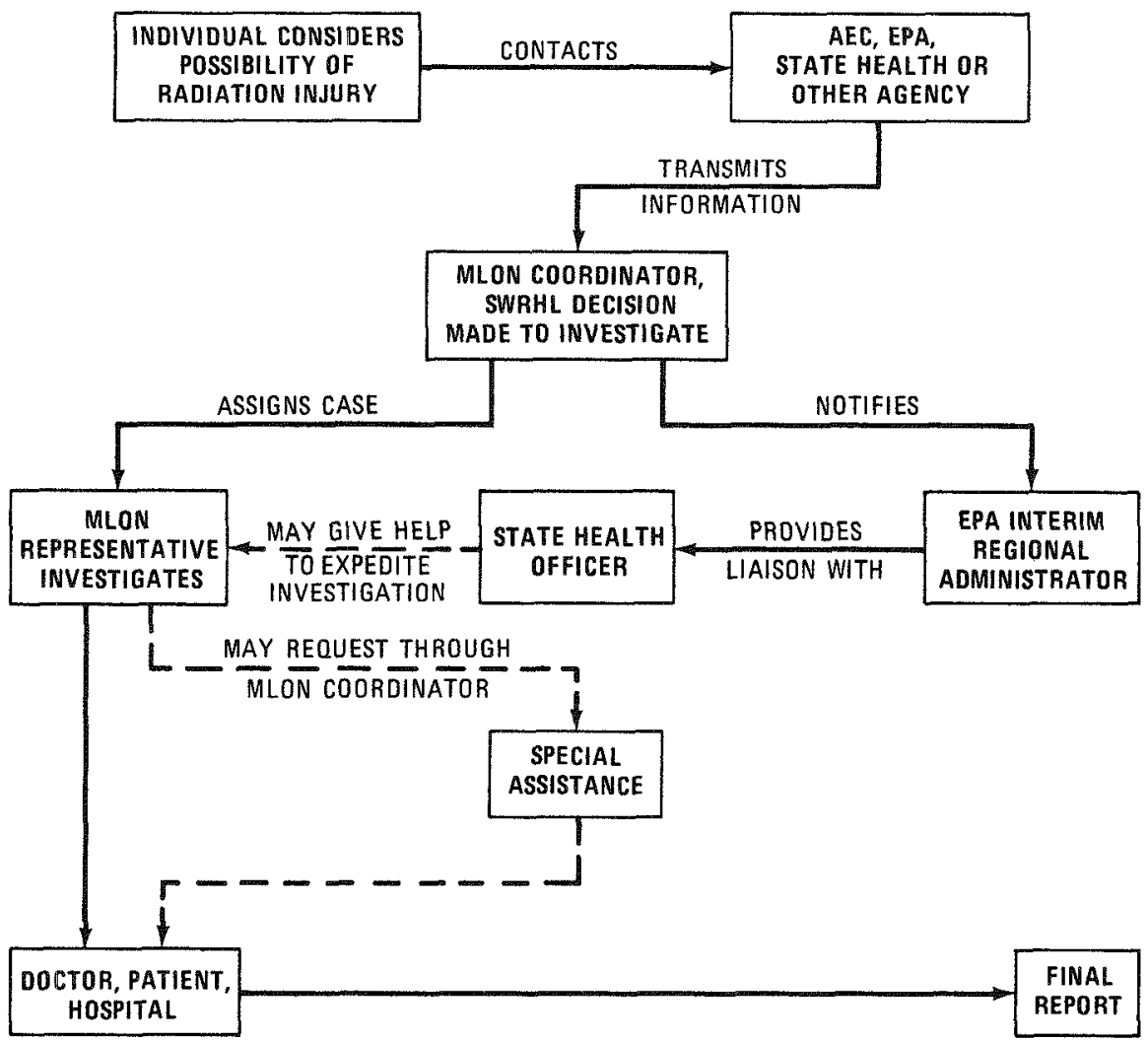

Fig. 8.11 Investigation procedure for possible radiation injury to people.

mental radioactivity data for the time and location of the alleged exposure. It has been found that most cases end here, with the patient's physician helping the MLON physician to explain why the particular injury was not due to radioactivity.

Should additional assistance be needed, the MLON physician is authorized to utilize local laboratory and hospital facilities (or both) in order to obtain information which will enable him to make a definite diagnosis. Experts in radiation injury or other medical subspecialties can be called upon after consultation with the MLON Coordinator.

The MLON Coordinator keeps the Interim Regional Administrator of the Environmental Protection Agency and State Health Officers informed of the progress of every case. In this way liaison can be maintained with the appropriate state's health organization. 
Upon completion of the investigation, a report is sent to the U.S. Atomic Energy Commission and to the agency initiating the inquiry.

Since 1965 a total of some twenty inquiries have been handled by the MLON. As of the end of 1970 , no case of radiation injury to nonoccupationally exposed individuals has been established. One claim of radiation injury, which was investigated by the Southwestern Radiological Health Laboratory physician and determined to be invalid, has been taken to court and legal proceedings are in progress.

\section{Veterinary Investigations}

The objectives of the veterinary (or animal) investigation program are to provide background information and answer inquiries concerning possible radiological effects on animals, and to resolve complaints by livestock raisers, wildlife management personnel, and others concerned with the welfare of animals. Inquiries are investigated by the Southwestern Radiological Health Laboratory, and the U.S. Atomic Energy Commission's Nevada Operations Office attempts to resolve any complaints that may be made.

A veterinarian from the Environmental Protection Agency (EPA) is in regular contact with local livestock regulatory officials, veterinarians, county agents, fish and wildlife officers, and other local agricultural leaders. They are kept informed of special nuclear events planned for the Nevada Test Site or elsewhere. These individuals are often able to resolve local problems, but they are asked to report to the EPA veterinarian any situations they cannot deal with themselves.

All possible claims or complaints concerning injury to stock and wildlife reported to the EPA veterinarian are investigated promptly. In order to reach a tentative diagnosis, the veterinarian carries equipment that permits him to measure physiological responses, to conduct routine field tests for various conditions, and to make a postmortem examination if necessary. But the final diagnosis must await the results of laboratory tests. If treatment appears desirable, a local veterinarian is called upon to provide the service. The findings and actions are reported to a representative of the U.S. Atomic Energy Commission.

Nearly all such investigations have been resolved by personal contact between the concerned person and the EPA veterinarian. No 
claim for radiation injury to animals from an underground nuclear test has resulted in litigation.

As part of the veterinary program to provide background information on the effects of radiation on animals, samples of tissues and forage are collected periodically from an experimental herd of range cattle which graze freely on the Nevada Test Site (Fig. 8.12).

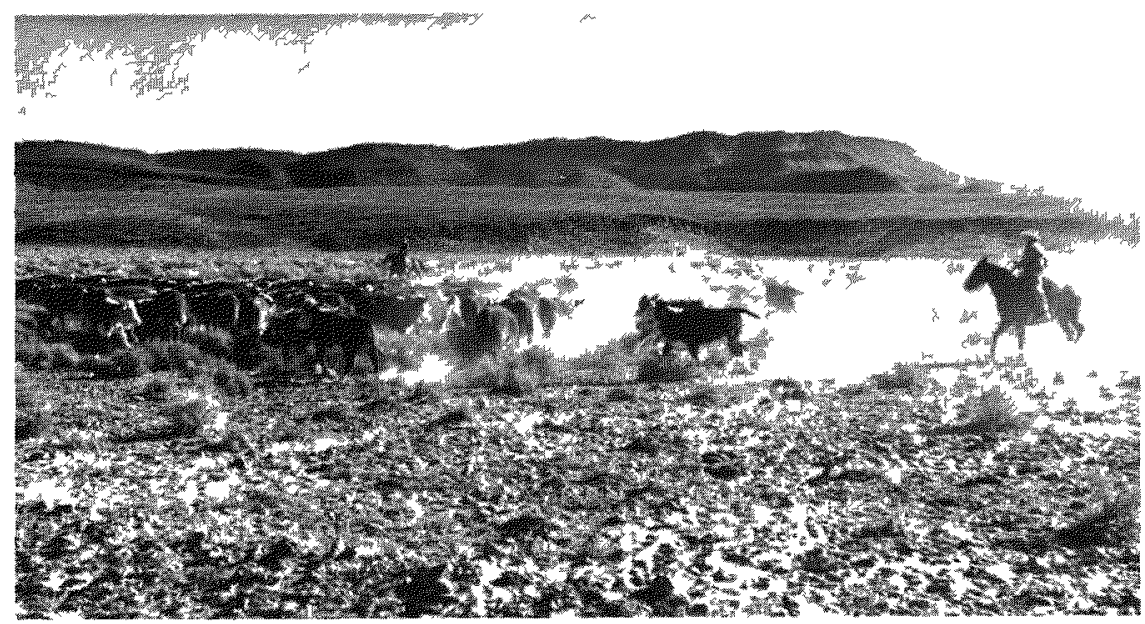

Fig. 8.12 Semiannual roundup of range cattle at the Nevada Test Site.

Management practices for this herd are comparable with general ranching practices in Nevada. The condition of the range cattle on the Test Site is similar to that of other herds in the state. Farm animals from other locations in the surrounding area and wildlife (e.g., mule deer) are sampled in a similar manner.

The concentrations of various radioactive isotopes in the samples have always been near the normal background levels except for those taken immediately after an occasional accidental release of radioactivity. Careful examination of the tissues collected postmortem from various animals has revealed no evidence of injury or damage from radiation.

\section{SUMMARY}

In underground nuclear detonations that are designed for containment, essentially no radioactivity escapes into the atmosphere and members of the general population are not exposed to radiation 
levels above the normal (natural) background. Only as the result of an accident is there a significant release of radioactivity. The purpose of the radiological safety program is to ensure that the public health is safeguarded should any radioactive residues from an underground detonation enter the atmosphere.

The Federal Radiation Council recommended Radiation Protection Guides and Radioactivity Concentration Guides for external and internal exposures, respectively, to radiation of the general population for normal peacetime operations. These guides have been used by the U.S. Atomic Energy Commission to formulate radiation protection standards. When conducting a nuclear detonation, every effort is made to keep radiation exposures as low as practicable.

The first indications of a possible release of radioactivity would usually come from a ground monitoring system (RAMS) within the Nevada Test Site. Aircraft flying over the immediate area above the detonation point carry instruments which would verify whether or not there has been a significant escape of radioactivity. If there has, the effluent is tracked to determine where monitoring on the ground for radiation may be necessary.

Measurements of radiation intensity (exposure rate) and of total accumulated exposure at various places on the ground around the Test Site would be made at frequent intervals, so that abnormal situations could be recognized immediately. Preparations will have been made in advance of the detonation for actions that might be required for the protection of people and domestic animals.

Samples of air, water, and milk are taken at a network of stations around the Nevada Test Site, some as far as 300 miles away, on a routine basis and analyzed for the presence of radioisotopes. Particular attention is paid to radioiodine in cows' milk, because a large proportion of iodine that enters the body is rapidly taken up by the thyroid gland. For detonations outside the Nevada Test Site, the procedures employed are similar to those used at this site but they are modified to suit the particular location and type of detonation. Programs have been established to investigate promptly all reported cases of possible radiation injury to people and to domesticated animals and wildlife. 


\title{
Chapter 9
}

\section{THE \\ BIOENVIRONMENTAL \\ SAFETY PROGRAM}

\author{
INTRODUCTION
}

\section{Objectives of the Bioenvironmental Program}

The safety programs described in the preceding chapters are aimed primarily at protecting people from direct injury and man-made structures from possible damage resulting from underground nuclear detonations. The bioenvironmental program discussed in this chapter is concerned with the possible indirect and long-range consequences of conducting underground nuclear explosions of all types, including those involving earth excavation (see Appendix). The effects considered include the following: potential exposure of people to internal radiation by way of food chains; damage to wildlife populations and to vegetation; and general disturbances that may lead to undesirable changes in ecological systems (or ecosystems), that is to say, in the balance of the relationships between living things and their environment.

The bioenvironmental safety program deals with the potential impact of all the effects of a nuclear detonation, namely, ground motion, pressure changes in air and water, surface and groundwater contamination, and release of radioactive isotopes to the atmosphere, on ecological systems. In addition, consideration is given to the ecological consequences of construction, site preparation, emplace- 
ment hole drilling, and other similar activities associated with carrying out the underground detonation.

In general terms, the program for safeguarding the environment has two main objectives which are interrelated. One is to identify food chain pathways, through plants and animals to man, that need to be monitored in the event of a release of radioactive materials to the atmosphere. The other is to avoid or minimize ecological changes, regardless of whether such changes arise from human occupancy of the detonation site, from construction activities, or from the direct effects of the explosion. In this regard, it is the aim of the U.S. Atomic Energy Commission to conduct underground testing in a manner that will ensure compliance with the National Environmental Policy Act of 1969.

In most cases, the physical disturbances resulting from operations in preparation for underground nuclear detonations have had more influence on the environment than the detonations themselves. However, such disturbances have been small in comparison with those accompanying the construction of a highway or a dam. The bioenvironmental effects of radiation from radioactive materials have been quite minor. Nevertheless, the ecological consequences of radiation are evaluated in order to make plans for protective measures should they become necessary after a nuclear event.

\section{Scope of the Bioenvironmental Program}

The operations for conducting a nuclear detonation will affect the surroundings to some extent. The approach adopted in planning an underground explosion is to estimate the possible ecological consequences in advance. If they appear to be detrimental, appropriate steps are taken to reduce or eliminate them where practical. In the final analysis, the costs of any unavoidable ecological effects must be weighed against the benefits to be gained by carrying out the nuclear experiment.

The bioenvironmental safety studies fall into the following four main categories: (1) Predictions of the potential effects of any given nuclear detonation on the ecological systems of the surrounding area. (2) Recommendations of courses of action to be taken to avoid or to minimize any harmful consequences that may be expected. (3) Evaluations of the ecological effects (if any) from the detonation. 
(4) Recommendations of corrective measures should there be any undesirable consequences.

Prediction of the effects of a nuclear detonation on the ecological system is, of course, of fundamental importance and more will be said about this later. One aspect of this activity is the identification of the components of the system that are most sensitive to disturbance. If a release of radioactivity is expected (e.g., from excavation explosions or from the initial flaring of natural gas), the environmental pathways of the radioactive isotopes through the ecosystems to man must be identified.

The sensitivity of many components of an ecological system may be strongly dependent on seasonal factors. An understanding of this dependence may suggest ways to minimize the effect of a disturbance. For example, if there is a bird population in the vicinity of a test area, it would probably be most seriously affected by a detonation during the nesting season. This would be taken into consideration in deciding upon the date for the nuclear event (p. 206).

Similar considerations may apply to the possible entry of radioactive isotopes into the bodies of people and animals by way of food chains as the result of an earth excavation explosion. The potential for such transfer might be high during one season but of lesser significance during another (e.g., when food crops are not growing).

\section{Dependence on Site and Event}

Because the problems to be dealt with are related both to the site and the event, bioenvironmental studies may vary widely in character and extent. The details will depend on the site location and the importance of the local ecological systems, and on the nature of the nuclear detonation.

When the device is exploded at a considerable depth, the probability of an escape of radioactivity is small. Nevertheless, the possible effects of radiation would be included in the bioenvironmental program in the unlikely event that a release to the atmosphere should occur. In experiments to stimulate the flow of natural gas, the probability of the escape of radioactivity at the time of the detonation is negligible, but it must be considered during subsequent drillback. In the course of flaring operations, some 
radioactivity is released to the atmosphere and the possible bioenvironmental effects are then taken into account.

Apart from any radiological aspects, the plans for a contained nuclear explosion of high energy yield may require a fairly extensive study of the ecological system. Such a study would be directed mainly at evaluating the sensitivity of the system to physical disturbance by effects related to the shock wave. The most important are ground motion, changes in terrain, and pressure effects in nearby bodies of water. This aspect of the bioenvironmental safety program received special attention in connection with the test site on Amchitka Island (see Fig. 1.3).

The discussion of bioenvironmental studies that follows is intended to cover all types of underground nuclear detonations in a variety of locations. A survey made before the event, taking into consideration the expected energy yield of the explosion, the purpose of the test, and the ecology of the area around the test site, will determine which aspects of the bioenvironmental safety program are essential in the particular circumstances. Of course, if the site is one that has been used previously for one or more detonations, the pretest survey will be less extensive than if a new site is involved, as it might be in a Plowshare experiment.

\section{DESCRIPTION OF THE ECOLOGICAL SYSTEMS}

\section{Delineation of Affected Area}

Bioenvironmental considerations enter into the process of selection of the sites for underground nuclear detonations. The sites preferred are those that appear, upon preliminary examination, to present the fewest problems from the standpoint of bioenvironmental safety; however, the actual site is likely to be selected on the basis of other overriding factors. After it has been chosen, a detailed assessment must be made of the potential ecological cost of the proposed event or events.

After the site is selected, approximate limits are set for the area in which ecological systems may be affected by the nuclear detonation. These limits are constantly re-evaluated as ecological data are collected and the bioenvironment situation is better understood. The local factors taken into account in deciding on the 
area to be included in the studies are site topography, hydrology, land usage, and animal, human, and plant population distributions.

A further consideration is the expected energy yield of the nuclear explosive; other conditions being equal, the area disturbed will be more-or-less directly related to the energy release. Moreover, both the yield and the depth of burial will determine the magnitude of the ground shock at various distances from the explosion site, the air blast in an excavation explosion (p. 234), and the probable areal extent of the fallout should there be an escape of radioactivity into the atmosphere (see Appendix).

\section{Ecology of the Area}

Once the area of bioenvironmental significance has been defined, the ecology of the area is characterized. The first step is to make a study of the available literature. Consultations are then held with biologists, fish and wildlife agents, agronomists, and others familiar with the region of the explosion site. The information obtained is supplemented where necessary by field studies. Efforts are concentrated on those aspects of the ecological systems that are expected to be most significantly affected by the nuclear test operation and on those components most likely to play a major role in the transfer of radioactive isotopes through the ecosystems should a release occur to the biosphere.

The methods employed in developing an ecological description of any site will depend on the characteristics of the ecological systems involved. Examples of such systems are agricultural lands, forests, grassland, bogs or marshes, streams, lakes and ponds, estuaries, open ocean, and so on.

The scope of the bioenvironmental study will consequently depend on the nature of the region where the detonation is to take place. At an inland site, the ecological system is largely (or wholly) terrestrial, although it may include a freshwater aspect if there are ponds, lakes, or rivers. For a site located on an island or near a seashore, on the other hand, marine life might be of primary importance. The subsequent discussion of ecological characterization will therefore be divided into two sections, dealing with terrestrial and aquatic (freshwater and marine) systems, respectively.

\section{Terrestrial Systems}

To characterize a terrestrial ecosystem, information is required on the distribution and composition of the major natural plant 
communities in the area. If any part of the area is under cultivation, similar information is also required about forage and food crops. This is especially important if a release of radioactivity to the environment is expected. Fallout may be intercepted by plants, and subsequently ingested directly by man and by herbivorous animals (e.g., cattle, sheep, and deer). Radioactive isotopes reaching the soil may also be taken up by plants through their roots and thus find their way into the food of people and animals.

Important food chains, including those in which humans are the ultimate consumers, must be identified and investigated. The food chain is traced through successively lower levels, often by examination of the stomach contents of animals, finally leading to the plant species of primary significance. Chemical analysis of samples taken at different stages of the food chain can provide data for computing the transport of radioactive isotopes through the ecological system, should this be necessary.

Part of the ecological description of a terrestrial system includes identification of the populations of living things that occupy the different habitats, that is, areas characterized by the plants and animals living there. For obvious reasons, attention is paid to those species that are eaten by man or those that constitute important economic resources in other respects. Consideration is also given to identifying rare or endangered species and others that merit special attention. In addition to knowing the nature of the main species, information is required about seasonal shifts in population density, migration patterns, and breeding habits of these species.

Another aspect of the bioenvironmental problem relating to plants arises when a considerable area is expected to be stripped of vegetation, for example, by site preparation activities. It may then be necessary to ascertain what pioneering plant species are available to invade the denuded area and how rapidly plant cover may be reestablished. Vegetative cover plays an essential role in regulating the runoff of rainwater and snow melt and in preventing erosion by wind and water.

\section{Aquatic Systems}

In studying the ecology of freshwater systems, such as ponds and lakes, it is desirable to know the rate at which organic matter is being formed by green plants, both floating and rooted. With the aid of the chlorophyll they contain, such plants are able to convert carbon 
dioxide into organic matter that serves as food for fish and other aquatic creatures. Thus, the primary productivity of the green plants, which can be measured by suitable experiments, is an index to the potential ability of the system to sustain consumer organisms.

Estimates of the populations of fish, shellfish, waterfowl, aquatic mammals, and other living things can be made, usually by direct sampling. Spawning areas are identified and seasonal sequences of spawning and egg hatching are determined by periodic examination of these areas. Food chain relationships are studied in essentially the same way as for terrestrial systems, working down to successively lower levels, when such detail appears to be justified.

Water chemistry has a bearing on the uptake of different elements by the various aquatic organisms. Radioactive isotopes would follow essentially the same paths as the stable isotopes of the same (or chemically similar) elements normally present in the water. Consequently, samples of water are collected and analyzed for those elements having radioisotopes that might be released in an underground detonation. Analyses may also be made for naturally occurring stable elements in order to predict how chemically similar radioactive species may behave in a water system. In addition, analyses may be made for nutrient elements, such as nitrogen, phosphorus, potassium, and others, that affect the growth of green plants and, hence, the primary production of organic matter referred to above.

For some locations, such as Amchitka Island, the bioenvironmental safety program also requires the investigation of marine ecological systems. The procedures used are, in general, similar to those described for terrestrial and freshwater systems. Particular attention is paid to marine species eaten by man, especially to commercially important fish that are harvested on a large scale.

In certain instances it may be sufficient to review catch statistics to ascertain the seasons of the year when the possible release of radioactive material to the ocean would be least likely to affect a commercial fishery resource. The event would then be executed in an off-season period, to allow time for the radioactivity concentration to decrease as a result of dilution and natural decay.

To study the dilution problem, information is obtained on ocean currents, transport rates, mixing processes, turbulence, and so on, for the area of bioenvironmental significance. The data may then be utilized to develop a model which will permit an estimate to be made 
of dispersion and dilution rates of any radioactive material that may enter the marine environment as the result of a nuclear explosion.

For detonations at sites close to a seacoast, consideration must be given to the potential effects of the shock wave in water on fish and their eggs and on other marine life. The effects of pressure changes in the water on marine organisms can be studied by using equipment specially designed to simulate the expected type of pressure pulse.

\section{ANALYSIS AND INTERPRETATION OF DATA}

\section{Introduction}

The large amount of data collected in making the ecological description of the detonation site in the manner outlined above must be subjected to systematic analysis and interpretation. In this way, useful predictions can be made of the bioenvironmental consequences of the planned nuclear explosion.

In a fairly complex ecological situation, the interpretative function involves application of the techniques of "systems analysis," which have been widely employed in recent years in connection with engineering and similar problems. Such procedures serve to utilize the available data in the most effective manner and also provide guidance concerning the types of ecological data and samples that need to be collected. There is consequently an interaction between the descriptive and interpretative aspects of the bioenvironmental safety program. The net result is an overall improvement in the predictive capability.

There are two main areas in which bioenvironmental predictions are required in connection with a proposed underground nuclear detonation. They are, first, the potential internal radiation pathways to man traversed by radioactive isotopes that may be released by the explosion and, second, the potential effects on plant and animal populations of radiation, direct shock, and other environmental disturbances. These two somewhat interrelated aspects of the problem will be considered in turn.

\section{Potential Internal Radiation Pathways to Man}

With regard to the possibility of radioactivity as an internal hazard to man (and animals), it appears that for most radioisotopes 
ingestion from food and water is more important than inhalation or absorption through the skin. For predicting internal radiation doses, and identifying the stages which should be monitored, a mathematical model can be constructed to represent the transfer of radioactivity from its source to man by way of all reasonable environmental and food-chain pathways.

A network of such pathways can be established by analyzing the dietary habits of the populations of interest and then determining the ecological system (or systems) from which the food and water originate. In a comprehensive study, a block diagram would be prepared showing the major possible pathways of material transfer in the vicinity of the explosion site. The general principles involved in investigating the fate of radioactive isotopes that might enter the ecosystem are described below. Although man is the end point of the model, information can also be obtained about the radioactivity in vegetation, animals, and aquatic life at intermediate stages of the food chain.

In the great majority of underground nuclear detonations, a much less detailed approach will be adequate. This is particularly true if the probability of appreciable release of radioactivity to the biosphere is small, as it would be in a deeply buried detonation. However, if radioactivity should enter the atmosphere as a result of postdetonation activities, due consideration would be given to the potential bioenvironmental effects of the radioisotopes released.

If a substantial release of radioactivity to a complex environment is to be expected, for example, as the result of a number of earth excavation explosions for the construction of a canal, the comprehensive model might be used. Considerable study of the populations and ecosystems would then be necessary, prior to the planned operation, to identify the potentially critical pathways, to determine the transport of various elements through the network, and to make numerical estimates of the coefficients upon which the rates of transfer through the various blocks of the network depend.

Bearing in mind the conditions under which it might be used, Fig. 9.1 shows an idealized network diagram for an area in which the human population obtains its water from streams and its food supply from terrestrial and aquatic systems in the vicinity of the explosion site. The blocks (or boxes) in the diagram are generalized to show the more important interconnecting stages in each ecological system and the principal pathways to man. In any actual situation, several of the 


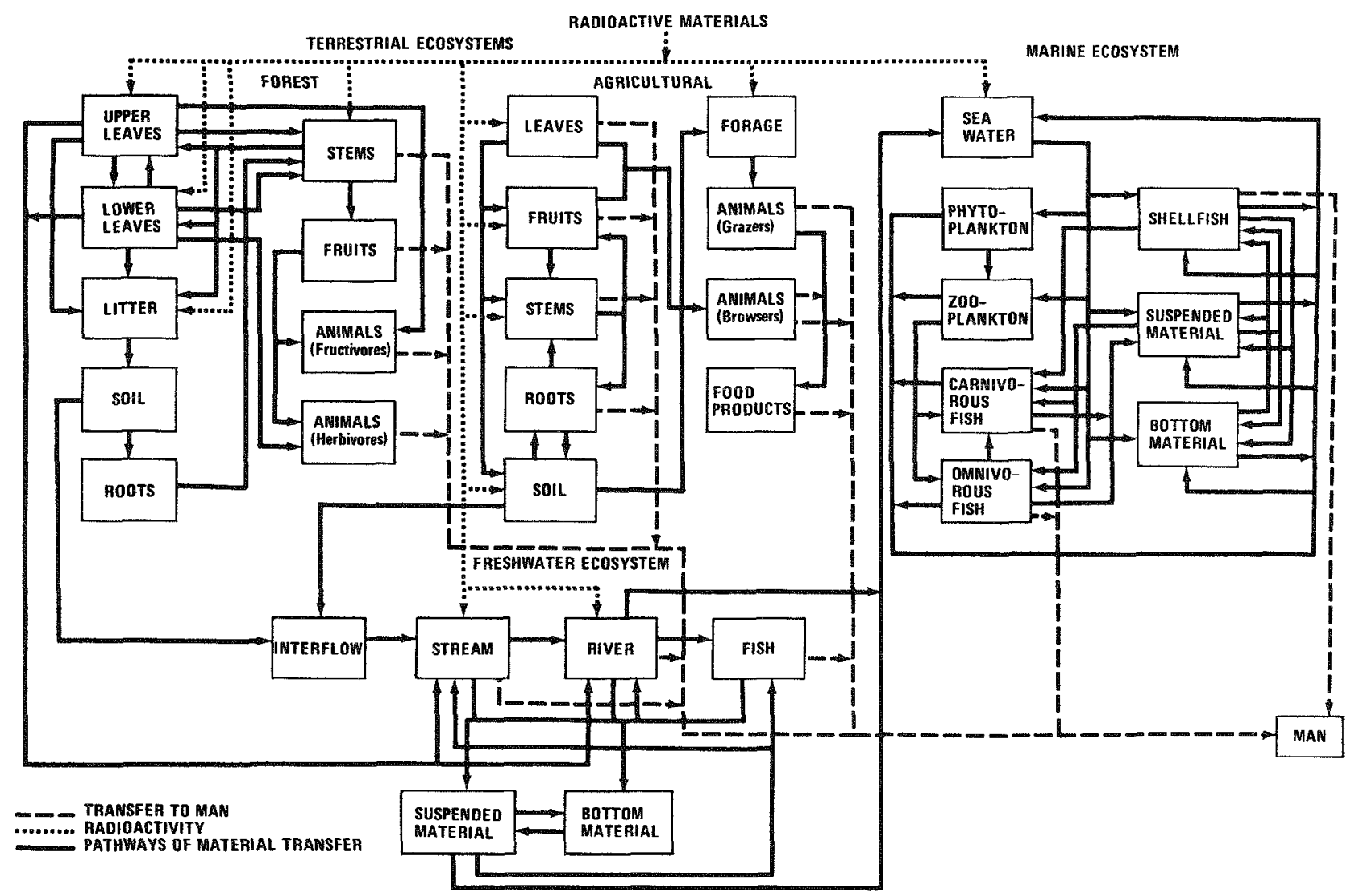

Fig. 9.1 Idealized network diagram of food pathways leading to man. 
indicated pathways might not be applicable and they would be omitted from the model.

When the model for the transfer of various radioactive isotopes from their source to man has been established, the next step is to represent the transfer through each block in the network by means of a mathematical equation. In order to do this, use is made of the data collected in the characterization and description of the ecological system.

The equations are programmed for solution by a computer. Sensitivity analysis, commonly used in such computations, will then identify the critical data and critical pathways under the existing conditions. This provides, on the one hand, a means for simplifying the network and, on the other hand, it indicates where better ecological data would lead to more accurate predictions. Steps can then be taken to secure such data if needed.

Apart from the ecological data that provide the input and output transfer coefficients for each block in the final mathematical (transfer) model, the only input information required is the amount and composition of the expected sources of radioactivity. The computer output will then be a prediction of the quantities of the various radioactive isotopes that may be expected to be present at each stage at a given time as the result of a particular nuclear detonation. If any hazard is indicated by the model, the critical stage (or stages) in the network can be identified where precautionary action taken before the detonation (e.g., advice to avoid certain foods) would be most effective.

It may take some time for radioactive isotopes released in a nuclear explosion to make their way through the various ecosystems. Consequently the transfer model could be used after an event to determine the fate of the isotopes in the ecological systems. In this case, the actual amount and composition of the radioactive release could be obtained from actual measurements and the final results would be more accurate than those based on a predicted situation.

The application of a comprehensive mathematical model, such as the one outlined above, requires a considerable knowledge of the populations and ecological systems in the area under study. When this information is available, accurate indications can be obtained of the critical internal radiation pathways for a wide variety of possible circumstances arising from an underground nuclear detonation. In the great majority of cases, considerable simplification is required and reliance is placed on predictions made in a less involved manner. 


\section{Potential Effects on Plants and Animals}

The potential effects of an underground nuclear detonation on populations or communities of plants and animals and on their physical environments (habitats) can be attributed to two main factors. These are the possible release of radioactivity to the biosphere and mechanical disturbances resulting from site preparation, emplacement hole drilling, and the detonation itself. This is a wider range of effects than is usually considered in assessing the potential consequences of the nuclear explosion to man. Only those plants and animals that are close to the detonation site are affected by mechanical disturbances. The area involved and, consequently, the extent of the bioenvironmental studies are therefore generally small.

The potential radiation doses to plant and animal populations can be estimated by the same general transfer model as that described above with man as the end point. As already mentioned, the computations in the latter case will give the doses for those plants, animals, fish, etc., which are part of man's food chain.

The radiation doses received from radioactive residues by plants and animals may be greater than for man. In the first place, plants may grow in areas close to the explosion site, from which man may be excluded. In these areas the radiation intensity, from both gamma rays and beta particles, is largest. Second, radioactive material intercepted by vegetation often becomes directly available in the food of herbivorous animals.

Radiation protection guides, such as have been recommended for humans, have not been established for plants and animals. Nevertheless, the bioenvironmental program takes cognizance of any potential radiation hazards to entire species or to rare or commercially valuable plant and animal populations.

Methods for assessing the potential effects of mechanical disturbance of the environment are difficult to describe precisely. It has been seen that these effects will usually be confined to close-in areas and are, therefore, of limited concern even though the local disturbance may be severe. No generalized modeling has been attempted for this kind of problem, largely because it can take a wide variety of forms. Consequently, each situation is analyzed on the basis of the existing circumstances.

One bioenvironmental generalization, in particular, may be useful for planning purposes. In ecological systems characterized by a large 
number of species and levels in the food chain and by environmental conditions that are relatively uniform (e.g., a tropical rain forest), the animal populations are generally more stable than in systems having few species and in which the conditions are both more severe and more variable (e.g., maritime tundra). In the former case, each consuming species will have a wide variety of food choices, so that considerable stability is to be expected. On the other hand, in the second situation, there are only a few species and a limited choice of food; a disturbance at any level in the food chain could then have serious consequences to the higher levels.

In relation to the effects of nuclear explosions, the implication is that harm to plant and animal populations is likely to be most severe in ecological systems characterized by few species and harsh environmental conditions, whereas it will be least severe in systems having many species and mild conditions. In general terms, disturbance of an apparently simple ecosystem may result in drastic population responses and slow recovery following the disturbance. On the other hand, similar disturbance of a more complex ecological system may result in only minor population responses which may be followed by rapid recovery.

In certain cases the important bioenvironmental problems will be immediately obvious to the trained ecologist. An example is given on page 206 in connection with disturbance of the tundra on Amchitka Island. The vegetation grows slowly, because of the poor soil and the severe climatic conditions. Hence, recovery after any disturbance that leads to denudation will be slow. Specific efforts to hasten recovery are being considered for Amchitka Island.

The potential effect of shock waves and related phenomena on individual members of important wildlife species can sometimes be assessed directly by experiments. Significant responses may be delayed and they may arise indirectly from destruction of the habitat or from disturbances that may be detrimental to food supplies.

\section{Postdetonation Evaluation of Bioenvironmental Effects}

After a nuclear detonation has been performed, the ecological consequences of the event must be evaluated. In the first place, this evaluation provides a necessary check on the reliability of the predictions. It indicates whether or not follow-up measures are required and what they might be. Furthermore, by comparing the 
predictions with the actual ecological effects, the predictive capabilities for subsequent detonations can be improved.

If postdetonation activities are accompanied by the escape of radioactive material into the atmosphere, the early stages of the bioenvironmental program will be concerned with the analysis of the biota (i.e., animal and plant life) and of soil and water in the area. The results of these early studies will indicate if any of the components of the biota are accumulating radioactivity to an extent that may be hazardous to individuals or to the population at large. Subsequently, if they appear desirable, long-range studies would be made to detect ecological changes that are not obvious at first sight but which may, nevertheless, be important.

The bioenvironmental program is concerned not only with radiological effects but with all the consequences of an underground nuclear explosion. Hence, surveys made after a detonation include the effects of ground motion and other phenomena arising from the shock wave on plants and animals and their habitats.

\section{BIOENVIRONMENTAL STUDIES AT THE TEST SITES}

\section{The Nevada Test Site}

The Nevada Test Site is an arid region with an irregular precipitation pattern. In the southern part, the ecology is typical of the Mojave Desert (Fig. 9.2), whereas the northern portion, with its high plateaus, resembles the Great Basin Desert (Fig. 9.3). Within these, there are some regions of desert woodland, known as the Piñon-Juniper community. The area of the Test Site has always been very sparsely populated by humans. Immediately before it was taken over by the U.S. Atomic Energy Commission it was used to a limited extent as grazing land for cattle.

Ecological damage at the Nevada Test Site has resulted mainly from construction activities associated with the nuclear testing program. Apart from these unavoidable consequences, completely contained detonations, in which there is no detectable escape of radioactivity, have caused only minor environmental changes. The subsidences on the surface above some of the detonation points (p. 40) have caused local disturbances to vegetation by providing invasion sites for pioneer species, both local and exotic. In some 


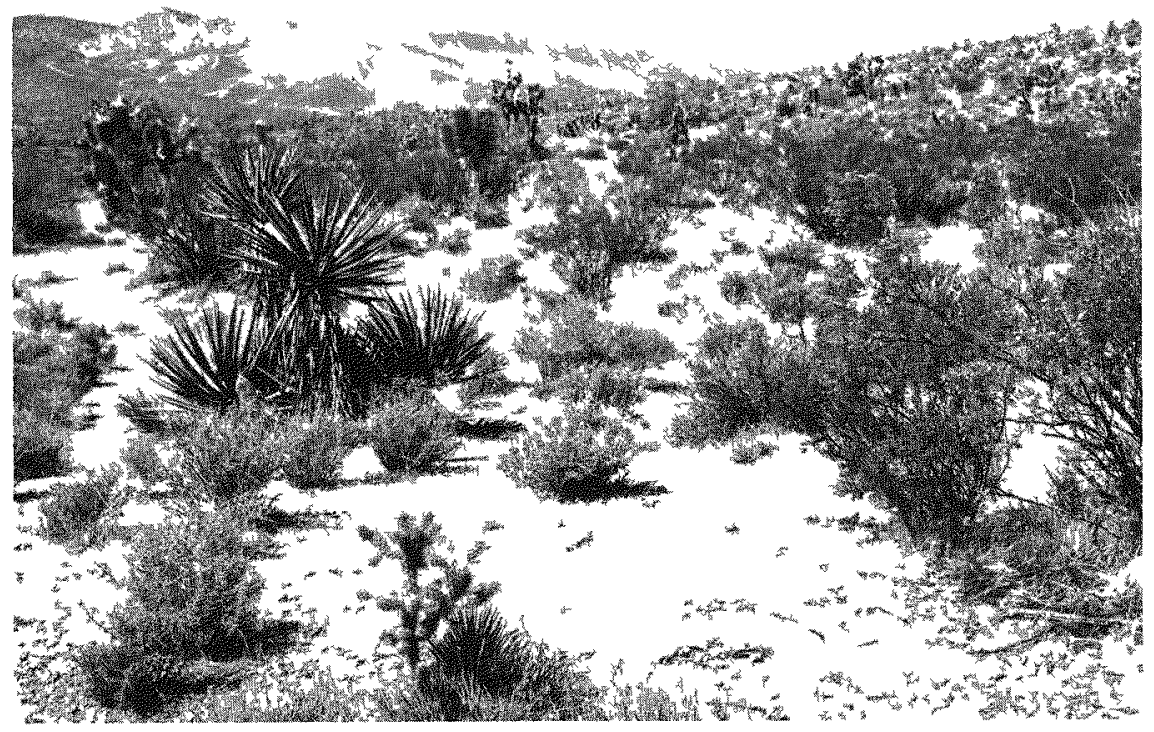

Fig. 9.2 Mojave Desert type vegetation at the Nevada Test Site

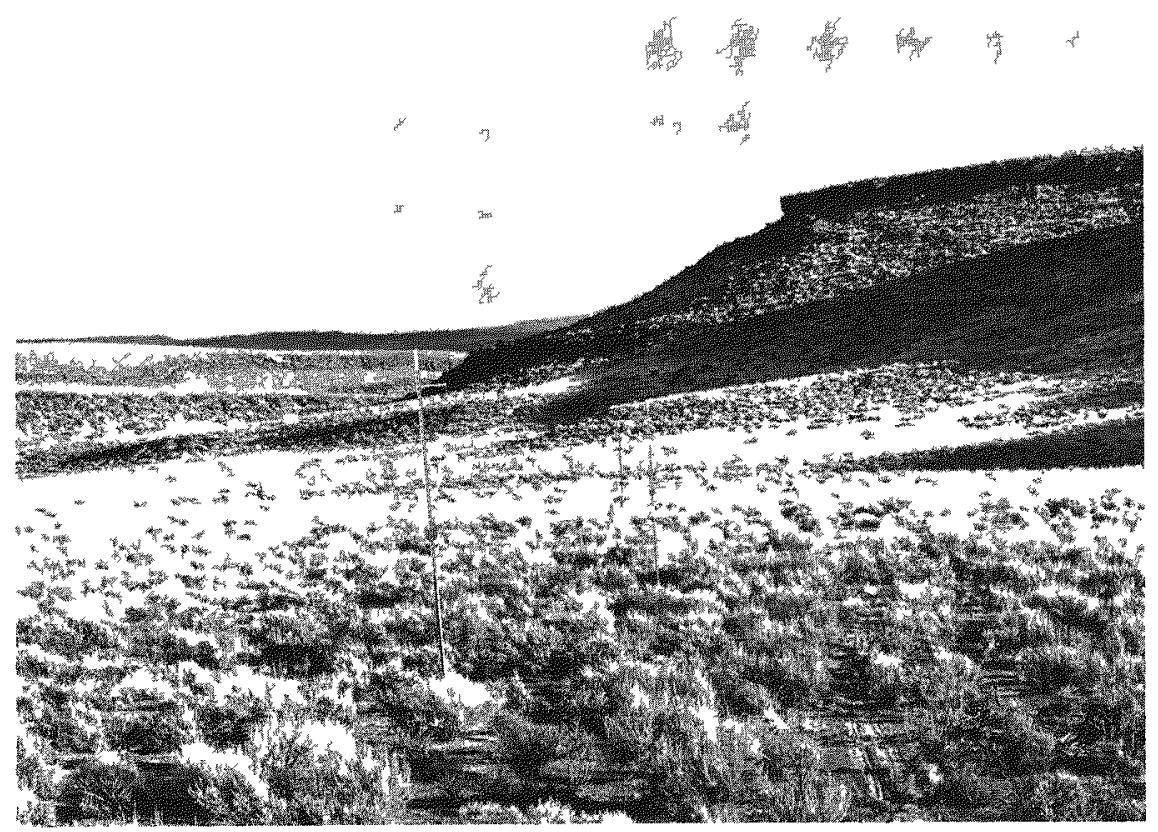

Fig 93 Great Basin Desert type vegetation at the Nevada Test Site 
cases the subsidences act as catchment basins for surface water. This has resulted in some soil changes by erosion and in the accumulation of salts due to evaporation. But the vegetation does not appear to have been affected to any marked degree.

Plowshare excavation tests have produced somewhat more disturbance to the bioenvironment because of the planned throwout of earth and the accompanying escape of radioactivity. The major visible effect has been to change the vegetation cover. Plants in the vicinity of the explosion site have been killed or injured as a result of smothering by the throwout and by radiation from close-in fallout, but the disturbed areas have been invaded by other species. Grasses, such as Indian ricegrass, have become more prevalent (Fig. 9.4) due to the absence of competition by shrubs.

These changes in the vegetation have undoubtedly affected the populations of small animals in the immediate area because of changes in their habitats and food sources. But the number of excavation tests has been small and so changes in the plant growth have not been extensive enough to have serious environmental consequences.

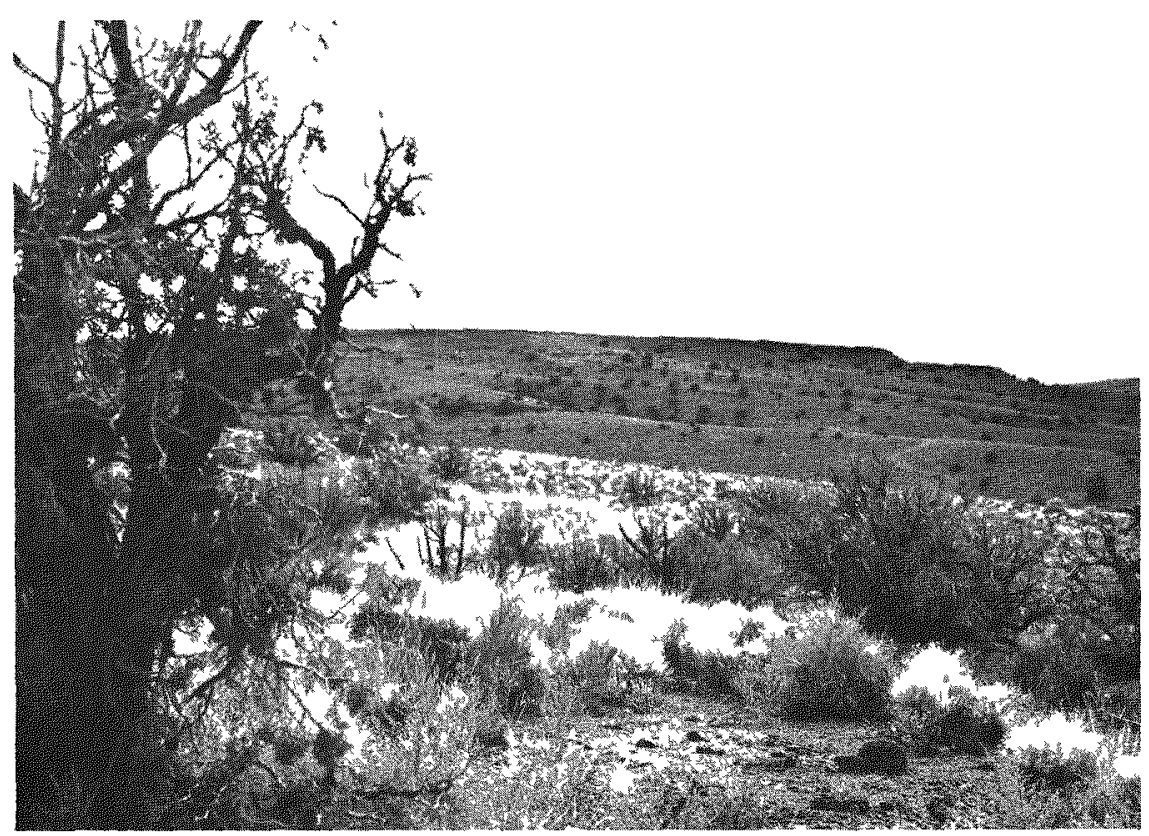

Fig. 9.4 Invasion of light-colored Indian ricegrass. (The trees and shrubs in the foreground were killed or injured by fallout.) 
Aquatic habitats are a minor feature of the Nevada Test Site. There are a number of springs and seeps, and after heavy rains the dry-lake beds are covered with water for a short period. No effects on these aquatic ecosystems have been identified.

Most of the changes in the environment caused by underground nuclear explosions have resulted from mechanical disturbances. The effects of radiation have been minor. During the early stages of underground testing, accidental leakage of radioactivity to the atmosphere killed a few piñon and juniper trees, located within a few hundred feet of the explosion area. In addition, some sagebrush shrubs (artemisia) and juniper trees, within a mile or so downwind, have been either injured or killed by the deposition of fairly large amounts of fallout from excavation experiments at the Nevada Test Site (see Fig. 9.4). It has been established that in these cases the damage was mainly due to beta-particle radiation rather than to gamma rays.

Since 1955, the Southwestern Radiological Health Laboratory has conducted an Animal Investigation Program in which mule deer and beef cattle (p. 186) from the Nevada Test Site are sampled on a routine basis for the presence of radioactivity. No unusual effects have been observed as a consequence of underground nuclear detonations. One result of these studies has been to show that the levels of such radioactive fission products as strontium-90 and cesium-137, present in the worldwide (delayed) fallout from atmospheric tests, are lower than in animals in wetter climates where these isotopes are carried down by rain and snow.

One consequence of the use of the Nevada Test Site by the U.S. Atomic Energy Commission is that some twenty-five organizations have conducted a large number and variety of ecological studies of the area. Research is under way to determine if there are any subtle, long-range bioenvironmental effects at the Test Site that may not yet be apparent. Active work in this connection is being done by the Ecology Division of the Laboratory of Nuclear Medicine and Radiation Biology, University of California at Los Angeles, by the Biomedical Division of the University of California's Lawrence Radiation Laboratory, and by the Environmental Protection Agency's Southwestern Radiological Health Laboratory, Las Vegas, Nevada, and by other organizations. Over two hundred reports dealing with various biological and ecological aspects of the Nevada Test Site have been written and many have been published in scientific journals. 


\section{Amchitka Island}

Long before any underground nuclear tests were contemplated for Amchitka Island, the environment had been disturbed both biologically and physically. Fur hunting since about the middle of the 18 th century led to the near extinction of the sea otter until this animal was protected by an international treaty in 1911 (p. 211). Blue fox farming was introduced in 1921 and hunting continued at least through 1936. The Island was occupied by up to about 10,000 members of the military forces of the United States from 1943 to 1951. Many hundreds of small buildings were erected and they are now in various stages of disintegration (Fig. 9.5). An extensive system of roads and trails also still remains. The Norway rat was inadvertently brought in during the military occupation, but the population was reduced and the foxes eliminated with poisons by the U.S. Fish and Wildlife Service from 1951 to 1957. Some bald eagles were poisoned at the same time, but the population apparently returned to normal within two or three years. Apart from man, the Norway rat is the only terrestrial mammal now on Amchitka Island.

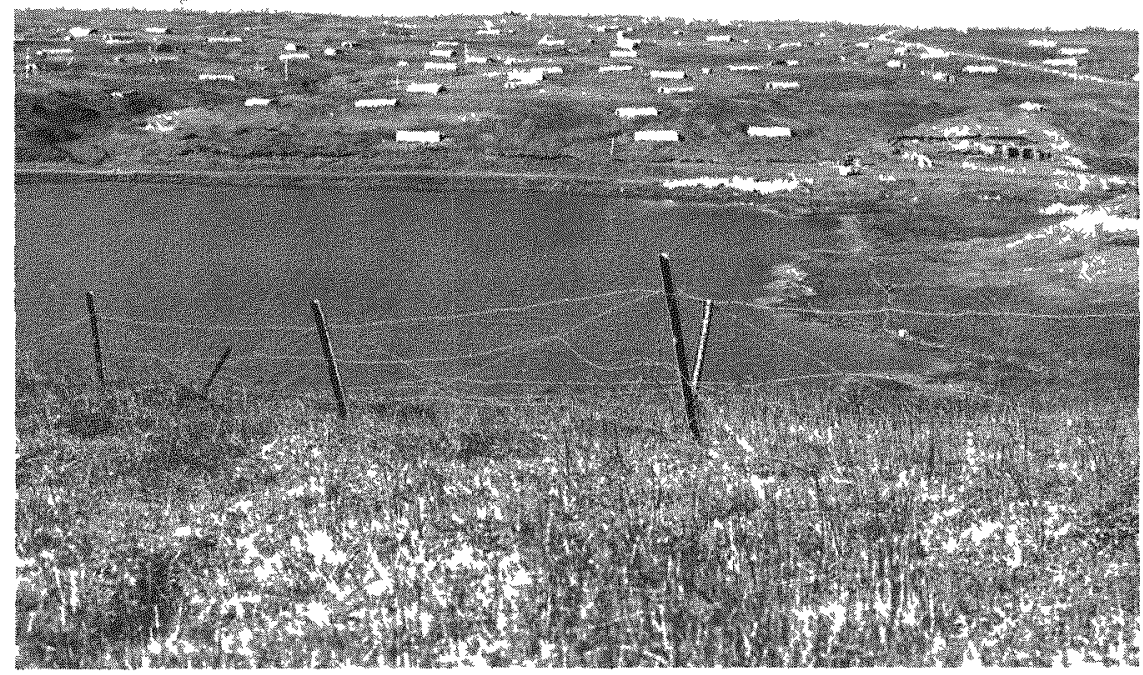

Fig. 9.5 Amchitka Island showing some of the buildings and roads remaining after the occupation by U. S. military forces. 
In October 1965, the Long Shot underground test, sponsored by the Department of Defense, was conducted on Amchitka Island (p.129), and some bioenvironmental studies were made in connection with this event. In 1966, the U. S. Atomic Energy Commission chose the island as a supplementary site for testing nuclear devices of high yield and operations started in the following year. A comprehensive program of bioenvironmental investigations was initiated at about the same time and has been continued since then under the coordination of Battelle Memorial Institute, Columbus Laboratories.

Much of the island is covered with a maritime tundra that is characteristic of the Aleutian chain. This consists of a thick mat of low-growing plants-grasses, sedges, lichens, mosses, and creeping shrubs - overlying a layer of spongy peat varying in thickness from a few inches to several feet. The tundra vegetation provides food and cover for birds that live or nest therein.

The mat of living vegetation is easily disrupted by vehicular and foot traffic. Since plants grow extremely slowly in the poor soil and harsh climate of the Aleutians, the vegetation of the tundra is fragile and slow to recover from physical disturbances. In fact ruts are still visible where vehicles were driven over the tundra of Amchitka Island during the military occupation. A certain amount of disturbance of the vegetation could not be avoided in the preparation for underground nuclear detonations on the island. Consequently, consideration is being given to the possibility of reseeding to hasten revegetation of the disturbed areas.

Two species of birds, the bald eagle and the peregrine falcon, which are classified as "endangered species" by the U. S. Department of the Interior, nest on Amchitka Island.* If the nesting areas are seriously disturbed, some birds may not nest again and their population will decrease. The nesting sites of these birds are mainly on sea stacks and sliffs (Fig. 9.6), whereas the nuclear test operations are largely in the interior of the island. Consequently, only a few nests were close enough to be affected. Nevertheless, the nesting season was one of the factors considered in choosing the date for the Milrow calibration test. This was conducted in October 1969, more than a month after the nesting season.

Another species that is rare elsewhere is the sea otter which is abundant in the waters around Amchitka Island and other islands in

\footnotetext{
*The bald eagle is not endangered in Alaska although it is elsewhere.
} 


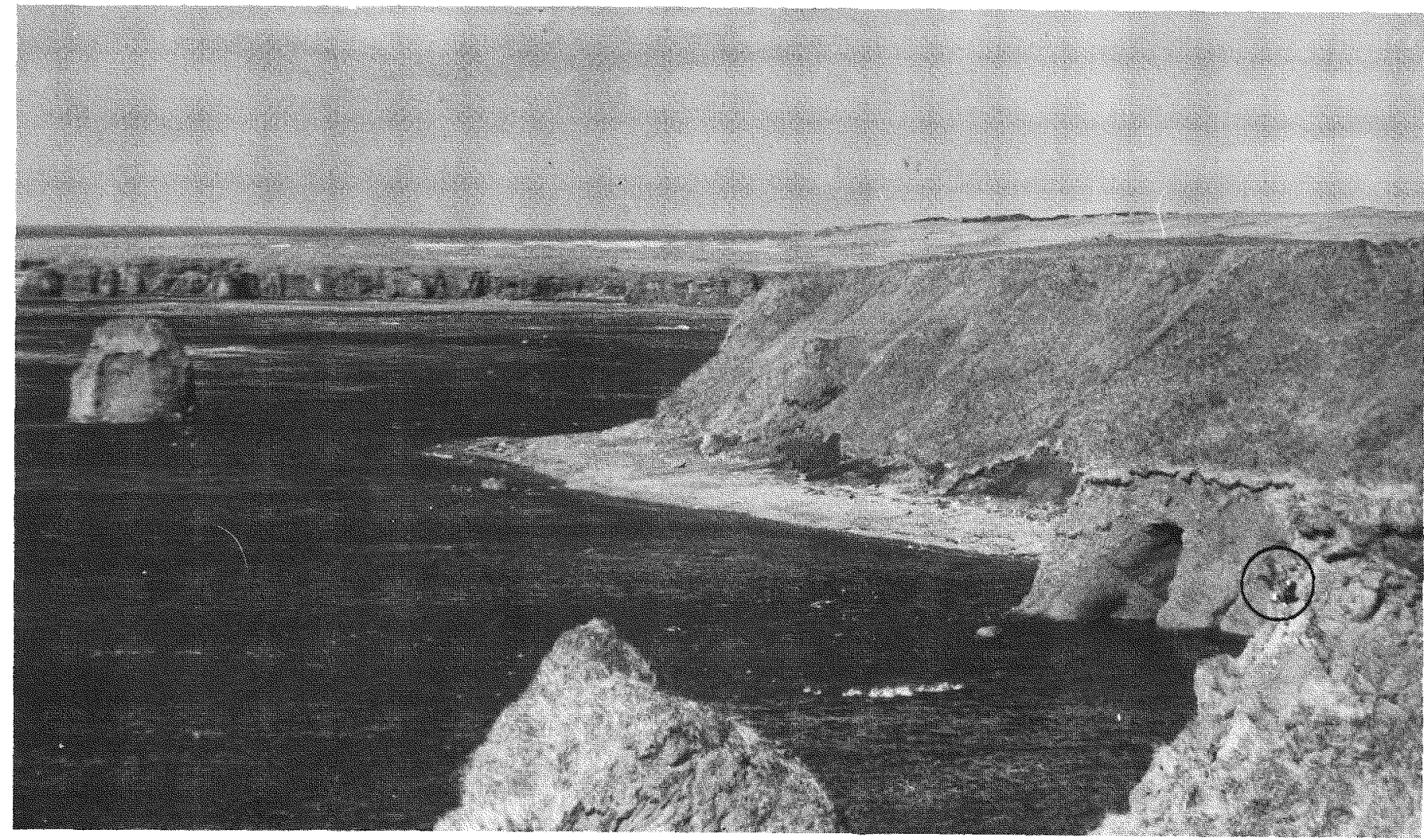


the Aleutian chain. Steps taken prior to the Milrow test in an effort to increase the numbers and distribution of this aquatic mammal are described on page 210 .

\section{Bioenvironmental Studies of Amchitka Island}

The bioenvironmental studies of Amchitka Island were designed to predict, document, and evaluate the potential effects of underground nuclear detonations and the activities associated with them on the island's plant and animal life, on its waters, and on the overall environment. An important objective of the program was to suggest how best to protect commercial marine fish (e.g., salmon, halibut, and Pacific Ocean perch), shellfish (e.g., king crab), marine mammals (e.g., sea otter), and birds (e.g., bald eagle, peregrine falcon, winter wren, and various water fowl), and the food chains and environments of these species.

The streams and lakes of Amchitka Island support only a limited number of fish used by man as food. The island itself is, therefore, not very important as a source of fish. The Dolly Varden (trout) is the only species of freshwater sport fish and a relatively small number of Pacific salmon which make a very minor contribution to the North Pacific salmon population spawn in the streams.

Commercial salmon fishing operations in the North Pacific and Bering Sea waters are conducted by Japanese fleets from about mid-May until early August. In addition, Japanese, Russian, and Korean fishing vessels are active in the area during the summer months and to a small extent in the autumn, fishing primarily for Pacific Ocean perch and halibut. Biological studies indicated that the minimum disturbance of marine (and freshwater) fish would result if the detonations were carried out in the autumn and winter months.

No escape of radioactivity to the atmosphere was expected from the Milrow event (and none occurred). Furthermore, calculations along the lines described in Chapter 4 indicated that, at worst, very low concentrations of radioactivity might eventually reach the Bering Sea by way of groundwater. An oceanographic survey was made to provide data for predicting the fate of any radioactivity that might enter the sea. The conclusion reached was that dilution would be so great that the radioactivity would probably not be detectable above the background. Nevertheless, an extensive monitoring program was established, both of seawater and of marine organisms, to check the predictions. 
Several months after the Long Shot test in 1965, radioactive tritium was found in three small ponds that had been used as sumps for drilling mud and in the drainage ditches from these ponds. The highest level of radioactivity observed was 13 picocuries per milliliter, which may be compared with the guideline value of 1000 picocuries per milliliter for drinking water used by the general public (p. 161). The manner in which the tritium entered the water is not known for certain. It is probable, however, that it seeped upward from the top of the rubble chimney formed by the explosion through the material used for stemming the emplacement hole. The depth of burial of the Milrow nuclear device (about 4000 feet) was much greater than for Long Shot and so seepage of radioactivity to the surface is less likely to occur. Nevertheless, surface waters on Amchitka Island are tested regularly to determine if the tritium exceeds the background level. Tests are also made for radioactive iron-55 and for isotopes that emit gamma rays.

Many studies of the flora and fauna of the island, including fish, animal, and bird population counts, were made visually and photographically. In order to observe the direct effects of the Milrow detonation on certain species, a number of Dolly Varden and salmon were penned in "live boxes" in several small lakes and streams on Amchitka Island just before the test. Similarly, several varieties of marine fish and crabs were placed in live boxes in the ocean, and sea otters were held in a pen located on the beach as well as in pens floating in the water (Fig. 9.7).

As a result of the bioenvironmental studies, it was predicted that, with the precautions taken, the effects of the Milrow explosion on living organisms and the environment would be minimal. This prediction was borne out by the surveys made after the event.

\section{Observation After the Milrow Event}

Observations made during shore walks and helicopter flights after the Milrow event revealed a number of dead sticklebacks (small freshwater fish) and three unidentified marine fish evidently killed by the explosion. Otherwise, there was no damage to fish, crabs, sea otters, or birds that could be attributed to the nuclear detonation. The sticklebacks, which were found in two lakes located about 2000 and 3000 feet surface distance from the explosion site, had apparently been killed by the effects of the shock wave from the detonation. 


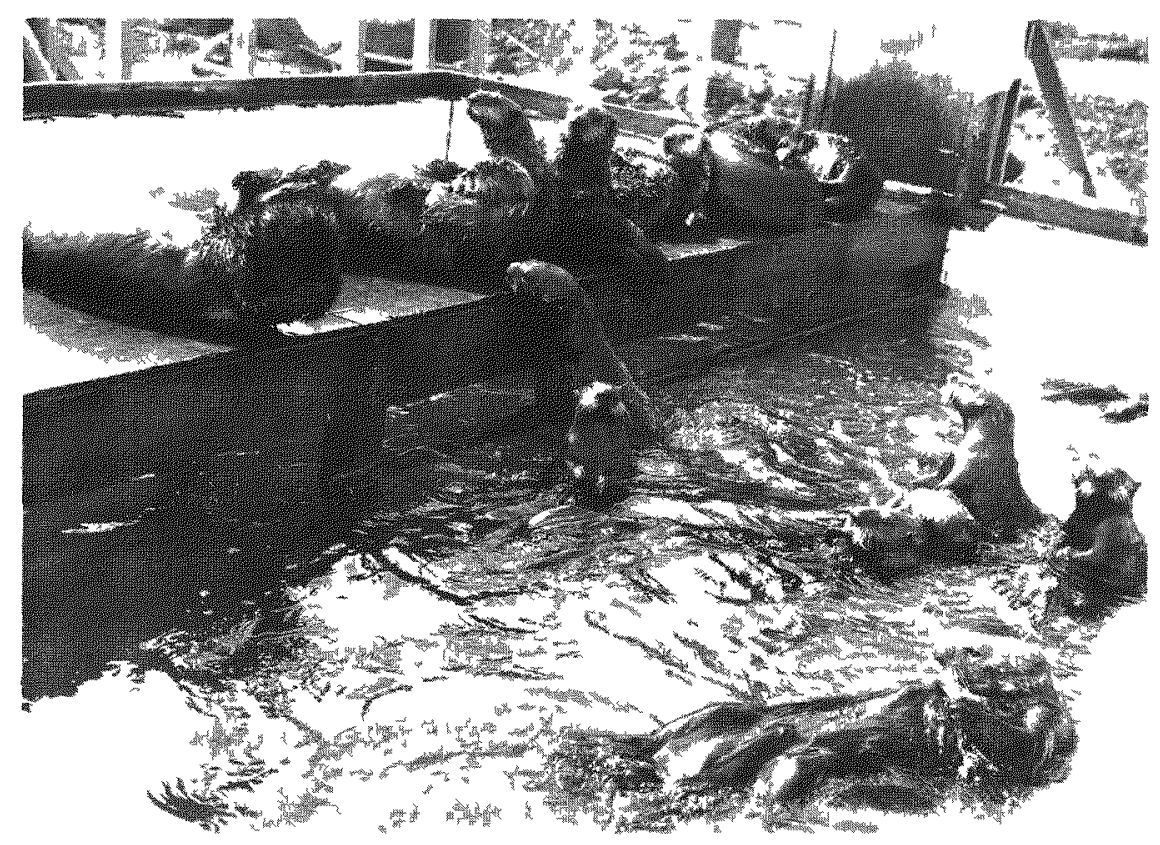

Fig. 9.7 Sea otters in a holding pen.

Several sea stacks close to Amchitka Island were damaged by the explosion but none were completely destroyed. The sea stacks are used as perches and nesting sites by birds, but the birds and their nests appeared not to have suffered at all. Eagles were observed sitting in pairs on damaged sea stacks within three hours after the Milrow test.

Aerial photographic surveys were made before and after the event to record changes, if any, in geology, vegetation, and wildlife. The only obvious effects (and these were minor) were seen in the geology of the coastal areas and in a few freshwater drainage systems. In addition to the damaged sea stacks already mentioned, rockfalls and peat and debris slides occurred, but nearly all were within two miles of the explosion site. Water in two ponds near to the site was partially drained by the subsidence in the ground caused by the explosion. All in all, the damage to the environment was quite small.

\section{Transplantation of Sea Otters}

The nuclear test activities on Amchitka Island were not expected to have any noticeable effect on the local sea otter population, as 
indeed proved to be the case. Nevertheless, preparations for the Milrow event may actually have had a beneficial effect in this respect.

The Aleutian Islands were at one time a major source of sea otters whose pelts were valuable in the fur industry. But, as a result of indiscriminate slaughter during the past two centuries, the population was so depleted that the animal had almost become extinct. In 1911, the United States agreed to an international treaty to protect sea otters and fur seals. Consequently, with a plentiful food supply, the sea otter population around Amchitka Island increased until it reached approximately 4500 in the early 1940's.

Such a large number of sea otters overtaxed the available food sources and the population declined, so that some twenty years later it had decreased to about 3000. Since the waters around Amchitka Island probably cannot sustain a stable population larger than this, the State of Alaska has been anxious to start new colonies of sea otters at other places where these animals once lived in considerable numbers. The development of the nuclear weapons test site at Amchitka Island provided the opportunity to achieve this objective.

With the help of the U.S. Atomic Energy Commission, from 1968 to 1970, the Alaska Department of Fish and Game transplanted about 650 sea otters from the Amchitka waters to eleven colonies in some of their former habitats in southeast Alaska, in the Pribilof Islands, and in Washington, Oregon, and British Columbia. Several years must elapse before the fate of these new colonies can be determined, but with plentiful food and careful protection there is every reason to believe that the sea otters will do well in their new homes.

\section{SUMMARY}

The bioenvironmental safety program in connection with underground nuclear detonations is concerned with all types of disturbances that may upset the ecology, that is, balance of living things and their environment. With regard to possible effects on plant and animal populations, consideration is given to ground motion, pressure in water, air blast, and radiation from radioactive materials that may be released into the biosphere. The pathways of such materials through the ecosystems to man must be traced in order to identify stages that need to be monitored. 
After a site has been tentatively selected for a nuclear detonation, the area in which disturbance of the biological environment may occur is delineated. A study of this area is then made to characterize the ecological systems. Special attention is paid to species which are of commercial importance or which are rare or endangered. The modes of transfer of materials through food chains, from the lowest stages to man, are investigated in the event that there is a release of radioactive isotopes to the biosphere as a result of the nuclear detonation.

On the basis of these studies, the expected bioenvironmental effects of site preparation, emplacement hole drilling, and the underground detonation itself are predicted. Recommendations are made, where necessary, to avoid or at least to minimize the potentially harmful ecological consequences. After the detonation, surveys are performed to determine the actual effects. If any significant damage were to be observed, steps would be taken to correct the situation. Differences between expected and actual consequences of the explosion would be used to improve the prediction methods.

Most of the ecological effects of the underground nuclear testing program have resulted from construction and related activities in preparation for the events. In addition, some localized changes in the vegetation pattern at the Nevada Test Site have been caused by debris thrown out in excavation experiments. Bioenvironmental effects of radiation, which have been minor and restricted to areas near the explosion point, have been observed only at this Test Site. 


\title{
Chapter 10 \\ SAFETY CONTROLS \\ FOR \\ NUCLEAR DETONATIONS
}

\author{
INTRODUCTION
}

\section{Planning for Nuclear Tests}

The United States Atomic Energy Commission is responsible for public health and safety in connection with nuclear detonations. The safety responsibility is implemented through the Commission's Nevada Operations Office, which coordinates the safety planning. This office conducts such studies and reviews as are necessary to make reliable predictions of those aspects of nuclear detonations that could affect the safety of people and property and disturb the environment.

No nuclear test is performed until the U.S. Atomic Energy Commission is convinced that the event can be conducted safely. In fact, safety is the overriding consideration in reaching the final decision whether to conduct a particular detonation or not.

Underground nuclear explosions have been in the nature of experiments needed to provide essential information that cannot be acquired by other means. Most such experiments are planned by three of the U. S. Atomic Energy Commission's laboratories, namely, the Los Alamos Scientific Laboratory and the Lawrence Radiation Laboratory, Livermore (both operated by the University of California), and the Sandia Laboratories. These three laboratories may also 
cooperate with the Defense Atomic Support Agency (U. S. Department of Defense) in the execution of special experimental and effects tests. Certain Plowshare experiments off the Nevada Test Site involve collaboration between the Atomic Energy Commission and industrial organizations.

Twice a year the Atomic Energy Commission obtains general approval from the President of the United States for nuclear detonations planned for the subsequent six-month period. Specific Presidential approval is required for all tests of high energy yield (i.e., 1 megaton TNT equivalent or more), for all nuclear excavation experiments and for all nuclear explosions outside the Nevada Test Site.

The planning for a test involving a nuclear detonation begins a long time ahead of the ultimate date of the event. In developing these plans, the laboratory that designs the experiment decides how deeply the nuclear explosive device is to be buried, how the emplacement hole is to be stemmed (i.e., filled), and how the explosion will affect the medium surrounding it. These and other considerations that have a bearing on safety are thus an integral part of the plans for each nuclear detonation.

On the basis of design data from the laboratory responsible for the proposed experiment and other pertinent information, the U.S. AEC's Nevada Operations Office makes an exhaustive investigation of the safety aspects. Every test, large or small, simple or complex, is analyzed for potential risk. If it is determined that the experiment as planned involves an unacceptable hazard, the plans are revised to eliminate or control the potential hazard. The modified plans are then re-evaluated to ensure that they meet safety criteria.

\section{Safety Reviews}

In reviewing the expected effects of a proposed nuclear detonation, the Nevada Operations Office obtains advice, guidance, and recommendations from several panels, groups, and subcommittees. The members of these bodies have special knowledge and experience in such disciplines as radiobiology, geology, hydrology, seismology, ground motion, and structural response. In addition, basic information is provided by contractors in private industry and by appropriate U. S. Government agencies.

The number and extent of the safety reviews conducted prior to a given nuclear detonation depend on the particular situation. If the 
location for the proposed explosion site is in an area which has been used in the past, the conditions might be sufficiently well known so that some aspects of the safety review would not be required. In a new area, however, the reviews would be quite comprehensive. The guiding principle behind the safety evaluation is that of checks and balances. Details of the plan for the proposed experiment are scrutinized and may be revised several times by many different people from various points of view. Only when the U.S. Atomic Energy Commission is satisfied that the test can be carried out safely is the Nevada Operations Office authorized to conduct the event.

\section{SAFETY PROCEDURES AND REVIEWS}

\section{Nuclear Systems Safety}

The laboratory responsible for the proposed experiment first evaluates what is called the "nuclear systems safety." This involves safety of procedures associated with assembly of the nuclear explosive device, its transportation to the detonation site, and emplacement in a hole or tunnel. The proposed means for firing the device are included in the nuclear safety plans. Positive measures are undertaken to prevent an accidental or unauthorized detonation.

The nuclear systems safety plans are then reviewed by independent groups of individuals with considerable experience in firing systems, in handling nuclear devices, and in conducting detonations. These individuals constitute the U. S. Atomic Energy Commission's Nuclear Safety Survey and Study Groups. If considered necessary, the groups recommend changes to assure that the device can be emplaced safely and that there will be no premature nuclear detonation. In the whole history of the testing of nuclear weapons, since 1945, there has not been an accidental or unauthorized nuclear explosion anywhere.

Another aspect of conducting the event with safety is the selection of the depth of burial and the means for stemming the emplacement and instrument holes. The sponsoring laboratory makes recommendations, based on man-made and natural (geologic) conditions which may influence the containment of the radioactive residues from the nuclear detonation.

The proposals for depth of burial and stemming, as well as for possible casing and cementing of the emplacement hole, are reviewed 
by the Containment Evaluation Panel which reports to the manager of the Nevada Operations Office. The panel is composed of voting members and alternates from the three U.S. AEC laboratories, the Defense Atomic Support Agency, and the U. S. Geological Survey (Department of the Interior). There are two other voting members selected because of their expertise in geology, hydrology, and underground nuclear phenomenology. In addition, there are advisors from the Southwestern Radiological Health Laboratory of the Environmental Protection Agency; the Air Resources Laboratory, Las Vegas, of the National Oceanic and Atmospheric Administration (Department of Commerce); and Reynolds Electrical and Engineering Co., Inc.

For a detonation that is designed for containment, every aspect that might relate to the possible escape of radioactivity is reviewed periodically by the Containment Evaluation Panel as preparations are made for conducting the nuclear event. A study of the geological features around the explosion point is performed by the U.S. Geological Survey and the results are presented to the panel.

The drilling, casing, and grouting history of each emplacement and instrument hole is followed to make sure that there will be no man-made path through which radioactivity can escape into the atmosphere. Finally, the proposed stemming (and other closure) and sampling plans must be approved by the Containment Evaluation Panel.

The foregoing has referred more specifically to underground nuclear detonations performed at the bottom of a deep hole, as the great majority are. Reviews of the same general nature, as appropriate, are also made to assure containment when the nuclear explosive device is placed at the end of a tunnel in a hillside.

\section{Effects Evaluation and Safety}

The safety reviews related to the potential effects of a particular underground detonation depend, to some extent, on the circumstances. For selected nuclear explosion experiments, a comprehensive Effects Evaluation Report is prepared. If the event is to be carried out at the Nevada Test Site and the explosion yield is not very large, the expected effects will lie well within the range of experience based on many previous events. For an explosion that will probably result in no release of radioactivity, a limited evaluation of the effects will then be adequate. 
For Plowshare (and other) tests in areas not previously used, for detonations of high energy yield, and for unusual situations, a detailed study is required of the expected effects of the proposed detonation. The effects include those which may affect people and structures as well as those which might cause disturbance of the biological environment. The report contains predictions of the effects of the detonation, including some that are considered to be very improbable. Safety procedures and operational controls to make sure that the planned detonation can be conducted safely take these predictions into account.

The Effects Evaluation Report is based on information from six Scientific Management Centers. These centers provide technical coordination of various contractors assigned by the U.S. AEC Nevada Operations Office to study special effects. The six Scientific Management Centers and the areas in which their responsibilities lie are enumerated below:

SANDIA LABORATORIES, ALBUQUERQUE: Ground motion; structure response; air blast; mines.

U. S. GEOLOGICAL SURVEY: Geologic studies; hydrologic studies; radioactivity in groundwater.

LAWRENCE RADIATION LABORATORY: Seismic (earthquake and aftershock) activity.

LOS ALAMOS SCIENTIFIC LABORATORY: Tsunami formation.

NATIONAL OCEANIC AND ATMOSPHERIC ADMINISTRATION: Meteorology and weather prediction; cloud trajectories; fallout patterns.

NEVADA APPLIED ECOLOGY GROUP: Bioenvironmental effects.

The actual report for a given event is prepared by a team consisting of a scientist from one of the Scientific Management Centers and a qualified member of the Office of Effects Evaluation of the U.S. AEC Nevada Operations Office. The report is reviewed by competent authorities from the Operations Office and from the three laboratories, and also, where deemed necessary, by independent panels, such as the one described below.

In special circumstances, for example, when the underground nuclear detonation is to be carried out at a new site or an explosive 
device of high energy yield is involved, the safety program and effects predictions may be reviewed by an independent Panel of Consultants which reports to the Manager, Nevada Operations Office. The regular panel consists of experts, from universities and industry, on such subjects as radiobiology, geology, ecology, hydrology, structural engineering, geophysics, and soil and rock mechanics. Consultants in seismology, tsunamis, air blast, etc., are included as required.

In addition to the reviews for specific events, studies are made of safety problems in general. In this connection, two subcommittees have been appointed by the Nevada Test Site Planning Board, an advisory group to the Manager, U. S. AEC Nevada Operations Office. The Seismic Evaluation Subcommittee reviews the accumulated data on natural seismicity and aftershocks, on ground motions, and on the response of structures, and the Radioactive Effluent Subcommittee studies radiation monitoring data and advises on the long-term success in controlling the release of radioactivity.

From time to time, ad hoc panels of experts not connected with nuclear testing activities are appointed to consider the safety procedures and to advise on new or difficult problems. The recommendations made by these panels contribute materially to the capability for carrying out underground nuclear detonations in a safe manner.

\section{Environmental Statements}

In accordance with the National Environmental Policy Act of 1969 and the guidelines established by the Council on Environmental Quality, the General Manager of the U.S. Atomic Energy Commission has directed that the Nevada Operations Office prepare in advance statements concerning the impact of underground nuclear explosions on the environment. For nuclear weapons tests with yields of 1 megaton or less to be conducted at the Nevada Test Site or at the Supplemental Test Site in Central Nevada, an environmental statement is prepared on an annual basis. For weapons tests of more than 1 megaton energy yield, however, at these Test Sites an environmental statement is required for each individual test. Individual statements are also required for the following: nuclear weapons tests on Amchitka Island; nuclear excavation experiments; and Plowshare projects in areas outside the Nevada Test Site. 


\section{SOURCES OF SAFETY INFORMATION}

\section{Ground Motion}

The predictions of ground motion, as described in Chapter 5, are made by the ground motion contractor, Environmental Research Corporation, formerly of Alexandria, Virginia, now of Las Vegas, Nevada. The data from previous underground detonations, upon which predictions are based, are obtained by the Special Projects Party, Las Vegas, Nevada, of the National Oceanic and Atmospheric Administration's National Ocean Survey (formerly the U.S. Coast and Geodetic Survey). Thus, members of the Special Projects Party are continuously engaged in procedures relating to the measurement of ground motion (and structural response) for essentially all underground nuclear detonations.

When a nuclear event has been scheduled, instrumentation plans are submitted by the ground motion contractor and the structural response contractor (see below) to the Office of Effects Evaluation, Nevada Operations Office. These plans contain recommendations for the location of instruments for measuring ground motion and structural response. They are reviewed and altered, as may appear desirable. When finally approved, the instrumentation plans are carried out by the National Ocean Survey, Special Projects Party.

The data acquired by the instrument array are analyzed by the ground motion contractor, Environmental Research Corporation, and compared with predictions for that particular detonation. The comparison is used by this contractor to improve the methods for predicting ground motion at various distances from other explosions.

About two months before a planned event, the ground motion contractor submits a report of the expected effects of the detonation. This report includes predictions of peak displacements, velocities, and accelerations of the ground at all towns and cities where motion might be felt. At selected locations, predictions of the frequency content (Chapter 5) are also given. These predictions are used by the structural response contractor to evaluate possible effects of the ground motion on structures that might be affected by the underground detonation.

\section{Response of Structures}

Predictions concerning the response of structures are made by the structural response contractor, John A. Blume and Associates, 
Research Division, San Francisco, California. This contractor has had many years of experience in studying the effects on various types of structures of ground motion caused by earthquakes, wind, and other phenomena, and in designing structures to withstand such motion. The procedures used in making predictions of structural response, based on expected ground motions, are outlined in Chapter 5.

Just as data from previous detonations are necessary for predicting ground motions, so also are measurements of structural response required to develop and improve prediction methods. The structural response contractor suggests the buildings where instruments should be placed. These will include experimental structures on the Nevada Test Site (p.97), as well as high-rise (and other) buildings some distance away. The measurements are made by the Special Projects Party, National Ocean Survey.

An important aspect of the work of the structural response contractor is to predict the effects to be expected from the proposed nuclear detonation. A first step in this direction is to survey the structures in the area that might be subjected to significant ground motion. In addition, the geologic conditions in the same area are considered.

When nuclear detonation events are conducted at the Nevada Test Site, the structures and geology are already well documented. For an event in a new area, such as a Plowshare experiment or for a test of a device of high energy yield, a survey is made to establish the characteristics and monetary values of structures that may be affected by ground motion from the proposed explosion. Structures are identified by their type, height, age, condition, and dollar value. Sometimes cost estimates may be made of possible damage to individual buildings.

In the course of the survey described above, structures may be noted where the ability to withstand ground motion could be improved with relatively little effort. Suggestions are then made as to the measures which should be taken, such as reinforcing chimneys and bracing walls.

The structural response contractor also makes specific recommendations regarding the safety of persons in or around structures. For example, before the Rulison event in Colorado, people within 50 miles were warned not to be in potential rock fall areas or in precarious positions, such as on ladders, scaffolding, roofs, or high walls. Although the ground motion was not expected to be severe, it 
was thought that if a person were in a precarious position at detonation time, he might be startled and lose his footing.

The Mine and Well Inspection Program is carried out by the U. S. Bureau of Mines (Department of Interior) Denver Mining Research Center and the Petroleum Research Center, Bartlesville, Oklahoma. A field survey is conducted to determine the location, ownership, condition, and history of mines and oil and gas wells in the area that might be subjected to appreciable ground motion.

Predictions concerning the possible effects of the planned detonation on mines and wells are based on ground motion studies made by the ground motion contractor. Where necessary, recommendations are made for steps to be taken that will minimize potential damage. People working in mines and areas where rock falls might occur are notified in advance of the plans for the detonation and are advised to leave the danger area just prior to the event.

\section{Geology, Hydrology, and Water Contamination}

The U.S. Geological Survey develops the geologic, hydrologic, and related information needed to evaluate the safety aspects of an underground nuclear detonation. In addition, the University of Nevada's Desert Research Institute provides a consulting service on groundwater.

Specifically, faults or other discontinuities which may lead to the escape of radioactivity are identified. Aquifers (i.e., water-bearing rock formations) in the vicinity of the explosion point are located and indications are provided of the groundwater pattern in the area. If possible, the rate of flow of such water is determined.

Teledyne Isotopes, Inc., makes predictions relating to the transport of radioisotopes in groundwater, based on data obtained from the U. S. Geological Survey and their own laboratory and field studies. Predictions are made of the possibility of radioactivity from an underground nuclear explosion reaching points where water is used or discharged beyond the immediate test area (Chapter 4).

The U.S. Geological Survey has drilled several hydrologic test holes at various distances from nuclear detonation points in Nevada. No radioactive isotopes have been found, in amounts exceeding the normal background values, in the water from any of the holes. This supports the predictions made by the contractor referred to above. Incidentally, people living and working on the Nevada Test 
Site in those areas where there are distribution systems drink water obtained from wells located within the Site. Continuous monitoring for radioactivity has confirmed the freedom of the water from contamination.

\section{Seismology and Water Waves}

Stations for observing seismic activity are operated by the U.S. Geological Survey and by the National Ocean Survey, Special Projects Party, Las Vegas. In addition, the Earthquake Mechanism Laboratory of the National Oceanic and Atmospheric Administration, the Colorado School of Mines, and California Institute of Technology have installed instruments for measuring earth strain. Studies related to aftershocks from underground nuclear detonations are being made by the University of Nevada and the University of Utah.

The possibility of tsunami formation (Chapter 6) is reviewed by the Tamarin Committee comprised of members from the Los Alamos Scientific Laboratory, the Lawrence Radiation Laboratory, Livermore, and the Defense Atomic Support Agency. This Committee, which reports to the Manager, Nevada Operations Office, is concerned primarily with developing an understanding of water waves generated by explosions and earthquakes and planning for the measurement of waves at specific points (and times) of interest. Support in pertinent theoretical matters is provided by Tetra Tech, Inc., Pasadena, California; this contractor conducts theoretical and model studies of tsunamis and of their interactions at coast lines. The Defense Research Laboratories of the Delco Electronics Division, General Motors Corporation, Goleta, California, are responsible for developing, emplacing, and recovering instruments for an extensive water-wave documentatior program. The University of Hawaii is also active in developing deep-sea wave gauges for emplacement at Amchitka Island and elsewhere in the Pacific Ocean.

\section{Meteorology, Weather Prediction, and Radiation Safety}

Activities related to meteorological recording and research and weather prediction are performed by the Air Resources Laboratory, Las Vegas, Nevada (Chapter 7). The laboratory provides predictions of wind speeds and directions and also of possible downwind cloud 
passage and radiation exposures should radioactivity be released either accidentally or as the result of an excavation explosion (see Appendix). Fallout models are developed by the Air Resources Laboratory, Silver Spring, Maryland.

The Environmental Protection Agency's Southwestern Radiological Health Laboratory, Las Vegas, Nevada, maintains records of populations and food chains that could be affected by underground nuclear detonations, and develops plans for their protection in the event of a release of radioactivity. The laboratory also provides a variety of radiation monitoring and related analytical services in support of radiological safety (Chapter 8 ).

\section{Bioenvironmental Studies}

The Columbus (Ohio) Laboratories of the Battelle Memorial Institute have the responsibility for the technical coordination of studies that are required to ensure bioenvironmental safety on Amchitka Island. They also act as ecological advisors for other bioenvironmental programs. Observations on the response of soil, vegetation, and animals to underground nuclear tests at Nevada are made by the University of Nevada, College of Agriculture, Reno, by the University of California, and by other organizations. Specialized ecological problems are examined by the National Marine Fisheries Service (U.S. Department of Commerce), the University of Washington, Ohio State University, the University of Tennessee, the Utah State University, the University of Arizona, the University of Alaska, and the Smithsonian Institution.

\section{MANAGEMENT CONTROL FOR SAFETY}

\section{Authorization for an Event}

When the U. S. AEC Nevada Operations Office is satisfied that a particular event can be conducted safely, that is, it will cause no injury to people and no unacceptable ground motion or damage to structures or to the environment, the safety plans for the detonation are submitted for review and approval by the Headquarters Staff of the Atomic Energy Commission. The Manager of the Nevada Operations Office then requests authority to carry out the planned 
test. When the Commission is satisfied that the specific event can be conducted safely the required authorization is given.

\section{Test Manager's Advisory Panel}

Responsibility for operational safety and authority to conduct the test are delegated to the Nevada Operations Office Test Manager (or to his equivalent for some Plowshare experiments off the Nevada Test Site). The Test Manager and his staff are assisted by an Advisory Panel composed mainly of specialists in meteorology, radiation, fallout, and medicine. Members of the panel include representatives of the three U.S. Atomic Energy Commission laboratories, the Environmental Protection Agency, and the National Oceanic and Atmospheric Administration, as well as special medical consultants. The Chairman is a Scientific Advisor from one of the three laboratories or from the Defense Atomic Support Agency. The panel examines predictions of the effects of the event and evaluates the preparations and operational controls designed to minimize possible hazards.

The Operational Safety Plan for the test includes precautionary measures which will be taken to clear potentially hazardous areas and to avoid injury to persons and damage to property. For events at locations outside the regular Test Sites, these measures may include recommendations to residents close to the explosion area to disconnect gas and electricity supplies, to remove fragile objects that might be disturbed by the detonation, and to avoid being in precarious positions. When there might be rock falls or landslides over highways or railroads, vehicular traffic would be regulated at the time of the event. If there is a remote possibility that a dam might be damaged significantly by the underground explosion, plans would be made for prompt removal of persons living downstream, should it be advisable.

The Southwestern Radiological Health Laboratory contributes to the radiation safety aspects of the Operational Safety Plan in several ways. The laboratory obtains information about the numbers and locations of people and milk cows in the area around the Nevada Test Site. Plans are developed for protective action should such action be desirable (Chapter 8). There are also plans for measuring actual radiation exposures and for the surveillance of the environment for radioactivity, including the testing of air, water, and milk 
after the detonation if it should be required Somewhat similar plans, as are appropriate to the curcumstances, are prepared for events outside the Nevada Test Site

For unusual tests that present special problems, the Test Manager's Advisory Panel may meet several times in advance of the event to review its safety aspects In any case, for events at the Nevada Test Site, the panel always convenes the day before the test is scheduled ( $F$ ig 101) A weather and radiation briefing is presented and a thorough review is made to ensure that the requirements of operational and safety plans have been $1 \mathrm{mplemented}$

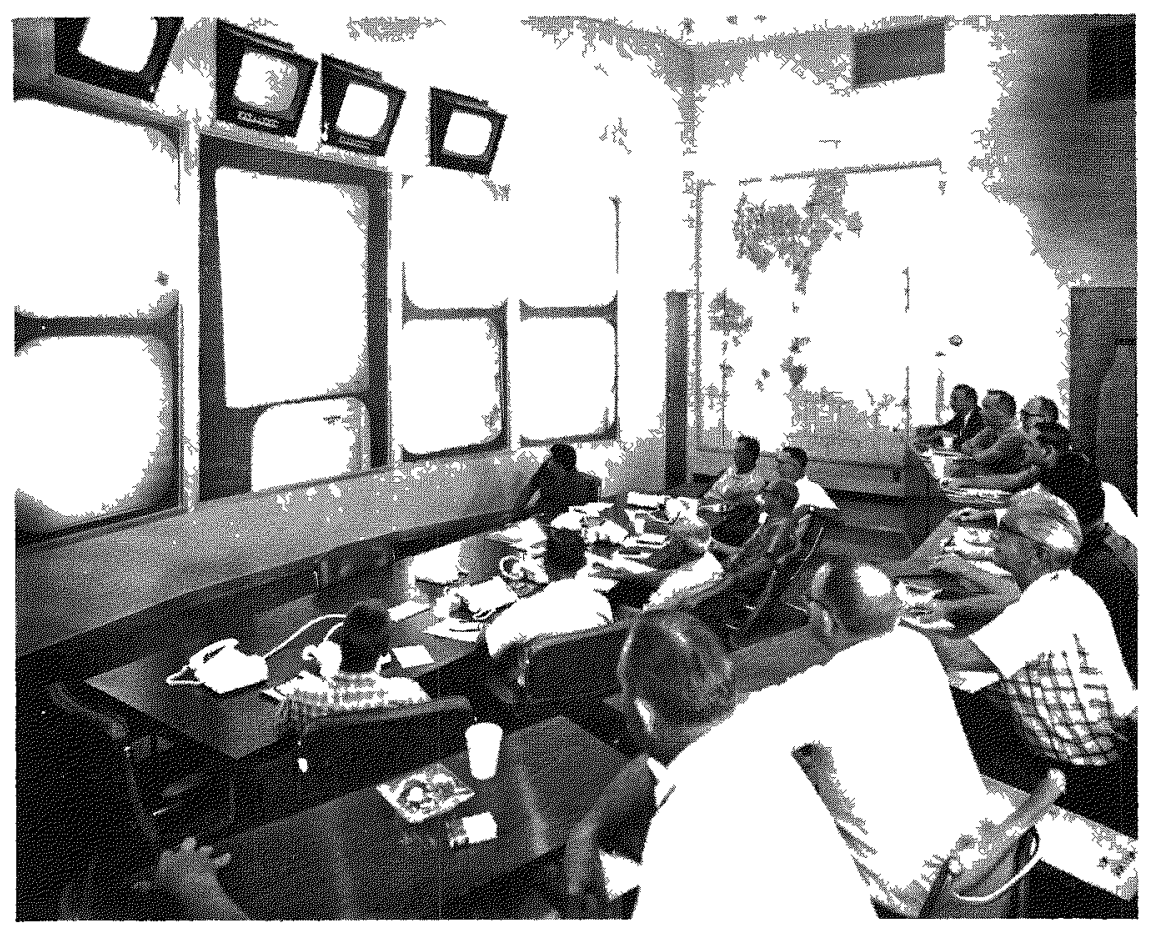

Fig 101 The Nevada Operations Office Test Manager's Advisory Panel in session before a nuclear detonation

If the detonation is not delayed by meteorological (or other) conditions, the Advisory Panel meets again on the day of the event, two or three hours before the scheduled detonation time If it is determined that the weather conditions are satisfactory and that the test can be conducted safely, assuming the hypothetical maximum 
credible accident, a recommendation is made to the Test Manager (or his equivalent) to proceed with the detonation.

The Advisory Panel remains in continuous session until the nuclear device has been fired and it has been verified that no radioactivity has escaped into the atmosphere and that nothing unexpected has occurred. However, at any time during the final countdown, up to the last second, the Test Manager can delay the event if any condition arises that might jeopardize the safety of people both on and off the Test Site.

\section{After the Event}

After the detonation, monitors of the Environmental Protection Agency make aerial radiation surveys to determine if there has been any escape of radioactivity beyond the limits of the Test Site. If there has, the trajectory of the radioactive cloud is followed in the air and at the same time extensive monitoring for radiation is carried out on the ground. Plans for environmental surveillance for radioactivity will also be implemented. Precautionary arrangements will have been made in advance, as mentioned earlier, to deal with any potentially adverse situation should it arise.

\section{CONCLUSION}

The United States' underground nuclear testing program has an excellent safety record. As a result of the planning, controls, and procedures outlined in this chapter, there has been no injury to persons and only minor damage to structures and the environment that could be definitely attributed to the detonations. Nevertheless, efforts are continuously being made to improve the capability for predicting accurately the effects of underground nuclear detonations. Techniques are constantly being improved to anticipate potential dangers and to avoid or minimize those which are unacceptable to the safe conduct of the testing program.

\section{SUMMARY}

The U. S. Atomic Energy Commission, with the collaboration of other Government agencies and the assistance of contractors from 
industry and individual experts, has established programs to ensure safety in conducting underground nuclear detonations. In fact, safety is the overriding consideration in reaching a decision whether to proceed with a particular event or not.

The safety programs and effects predictions are reviewed (and improved as may appear necessary) by appropriate experts before submission to the Headquarters Staff of the Atomic Energy Commission for approval. In special circumstances, a Panel of Consultants provides advice concerning safety aspects that need further consideration. Moreover, from time to time, ad hoc panels are appointed to review safety procedures.

For each event, the Test Manager (or his equivalent) is assisted by an Advisory Panel which includes specialists in meteorology, radiation, and medicine. The panel evaluates the safety plans and operational controls designed to avoid or minimize potential harmful effects of the explosion. Only when it is determined that a test can be conducted safely does the Test Manager proceed with the detonation. However, should the need arise, the event can be postponed as late as the final countdown. 


\section{Appendix \\ NUCLEAR EXCAVATION \\ SAFETY}

\section{CRATERING EXPLOSION PHENOMENA}

\section{Crater Formation}

The use of nuclear explosives for earth moving applications, commonly referred to as "nuclear excavation," depends on the formation of a "throwout" crater by an underground detonation at a suitable depth. Such a crater differs from a subsidence crater (p.40) and is produced as an indirect result of the rarefaction (or tension) wave reflected back into the ground from the surface (p.42). In a deep, fully contained explosion, the distance from the detonation point to the surface is large and the reflected wave is then too weak to have any significant effect. But at a somewhat smaller, although still substantial, depth of burial, the rarefaction wave can have important consequences.

The rarefaction wave causes the upper surface layers of the ground to spall, that is, to split off in more-or-less horizontal layers. As a result of the momentum imparted by the shock wave, these layers move upward. At a suitable depth of burial, when the shock wave and the reflected rarefaction wave are both fairly strong at the surface, the rate of upward motion of the spalled layers may be about 150 (or more) feet per second.

When it is reflected back from the surface, the rarefaction wave travels into the ground toward the cavity produced by the explosion. In a detonation at not too great a depth, this wave may reach the top 


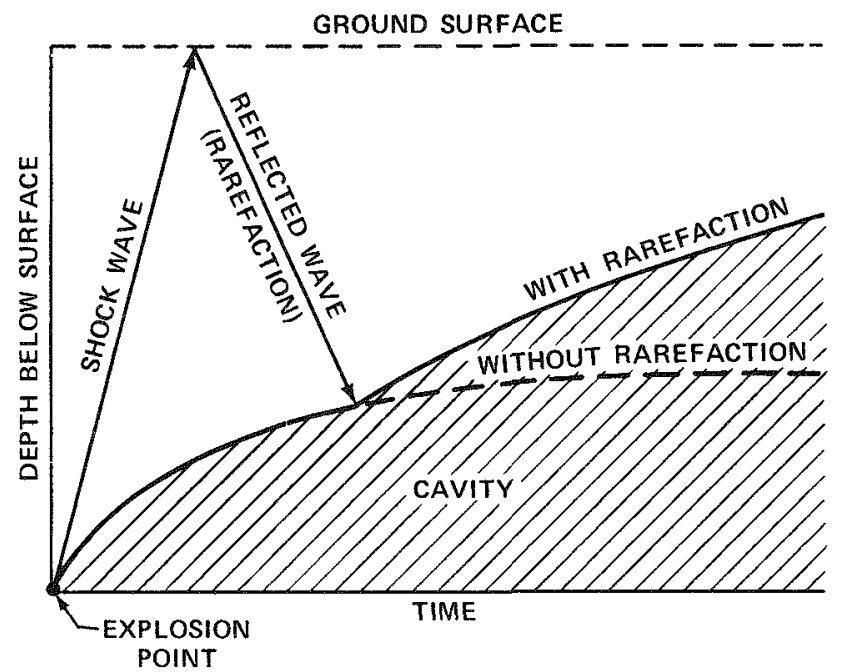

Fig. A.1 In a shallow explosion, the reflected wave reaches the cavity while it is still growing.

of the cavity while it is still growing. The external pressure resisting the upward growth of the cavity is then decreased to some extent, and the cavity expands rapidly in the upward direction (Fig. A.1). The expanding gases and vapors may thus supply additional energy to the spalled surface layers, so that they may move upward with increasing velocity. This velocity increase is called "gas acceleration."

The ground surface first assumes the shape of a dome, as a result of the upward motion. As the dome continues to increase in height, cracks form through which the cavity gases vent to the atmosphere within a few seconds. The mound then disintegrates completely and the rock fragments are thrown upward and outward. Subsequently, most of the ejected material collapses and falls back, partly into the newly formed crater and partly onto the surrounding "lip." The general term "fallback" is used to describe the material that immediately falls back in this way (Fig. A.2). The size of the remaining (or "apparent") crater depends on the energy yield of the explosion, the depth of burial, and the nature of the rock (see page 35 , footnote).

For each rock there is an optimum (or best) depth of burial at which an explosive of given yield will produce an excavation crater of the maximum size. If the detonation is too deep, much of the energy of the shock wave is expended by the time it reaches the 

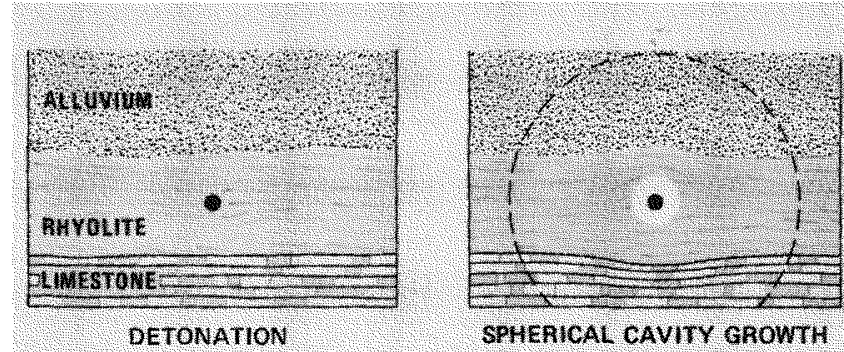

SPHEAICAL CAVITY GROWTH AND SHOCK FRONT

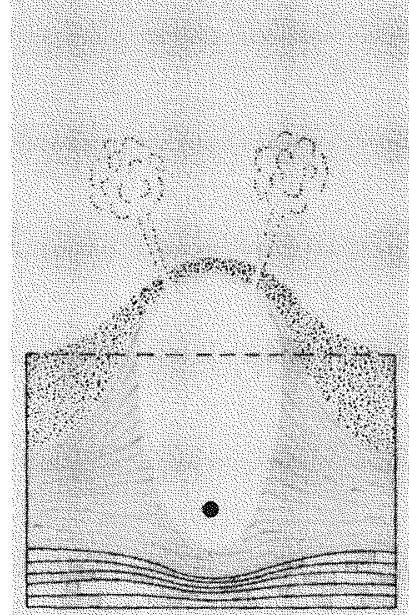

FIRST MAIOR VENTING

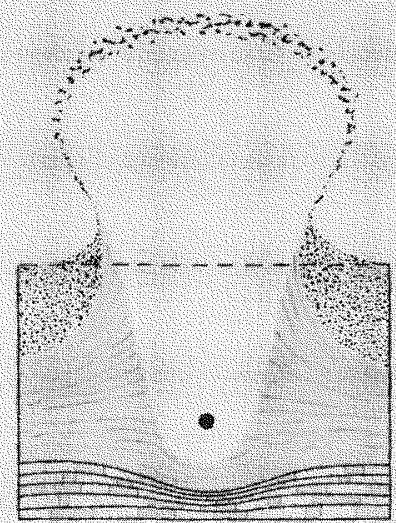

MOUND DISASSEMBLY
FOLD-OVER, AND

WITIATION OF COLLAPSE

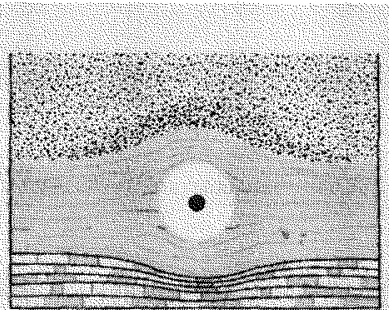

RAREFACTION RETURNS ro a AvTY

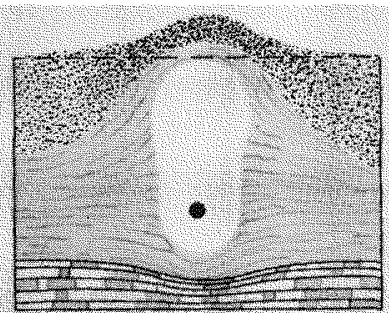

ASYMWE TRICAL CAVIT sfowth

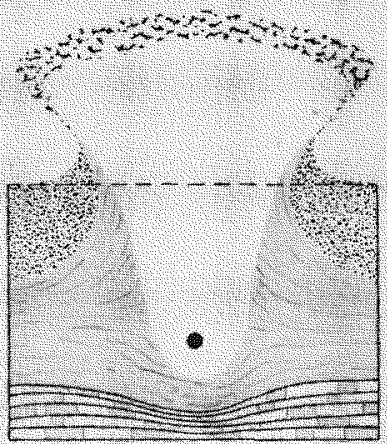

COLLAPSE AND TAL LBACK

Fig. A.2 Stages in the formation of an excavation crater. 
surface. On the other hand, if the explosion point is not deep enough, energy will be wasted in producing a blast wave in the atmosphere (p. 234). Calculations are made to predict the optimum burst depth for a set of assumed conditions that are believed to approximate the actual conditions of the detonation.

The nature of the rock can affect the mechanism of crater formation. If the medium has a low moisture content, the gas acceleration effect may be quite small, presumably because of the relatively low pressure of the gases and vapors in the cavity just before venting occurs. For example, in the Danny Boy test of March 1962, a low-yield (0.43-kiloton TNT equivalent) nuclear explosive was detonated at a depth of 110 feet in basalt, a hard rock, with a moisture content of less than 1 percent by weight. Observations of the ground showed that the upward motion was due almost entirely to spall. There was no significant increase in velocity when the gas in the cavity reached the surface. The apparent crater had a diameter of 214 feet and a depth of 62 feet.

In a rock containing a larger proportion of water than basalt, a considerable quantity of water is vaporized by the heat of the explosion. The vapor causes a large increase in the cavity pressure and then gas acceleration can make a significant contribution to the upward motion of the ground. In the Sedan excavation test of July 1962, a 100-kiloton nuclear (mainly fusion) device was exploded at a depth of 635 feet in alluvium containing 7 percent of water. In this case, the initial upward velocity caused by spall was definitely increased by gas acceleration. The resulting (excavation) crater, the largest explosion crater produced in the United States, is shown in Fig. A.3. The interior diameter of the apparent crater was more than 1200 feet and the depth 323 feet. The height of the lip ranged from 18 to 95 feet. The volume of the apparent crater corresponds to the removal of about 8.4 million tons of earth and rock.

The depth of burial, as well as the moisture content of the rock, can apparently affect the mechanism of crater formation. The Cabriolet experiment of January 1968 involved a 2.3-kiloton explosion in a dry (rhyolite) rock, but the depth of burial was less than the optimum for crater formation, such as was used for Danny Boy. Observations of the ground surface showed that gas acceleration contributed to the motion in the Cabriolet event, although it did not in the Danny Boy experiment. Thus, at less than optimum depths of burial gas acceleration may be favored. 


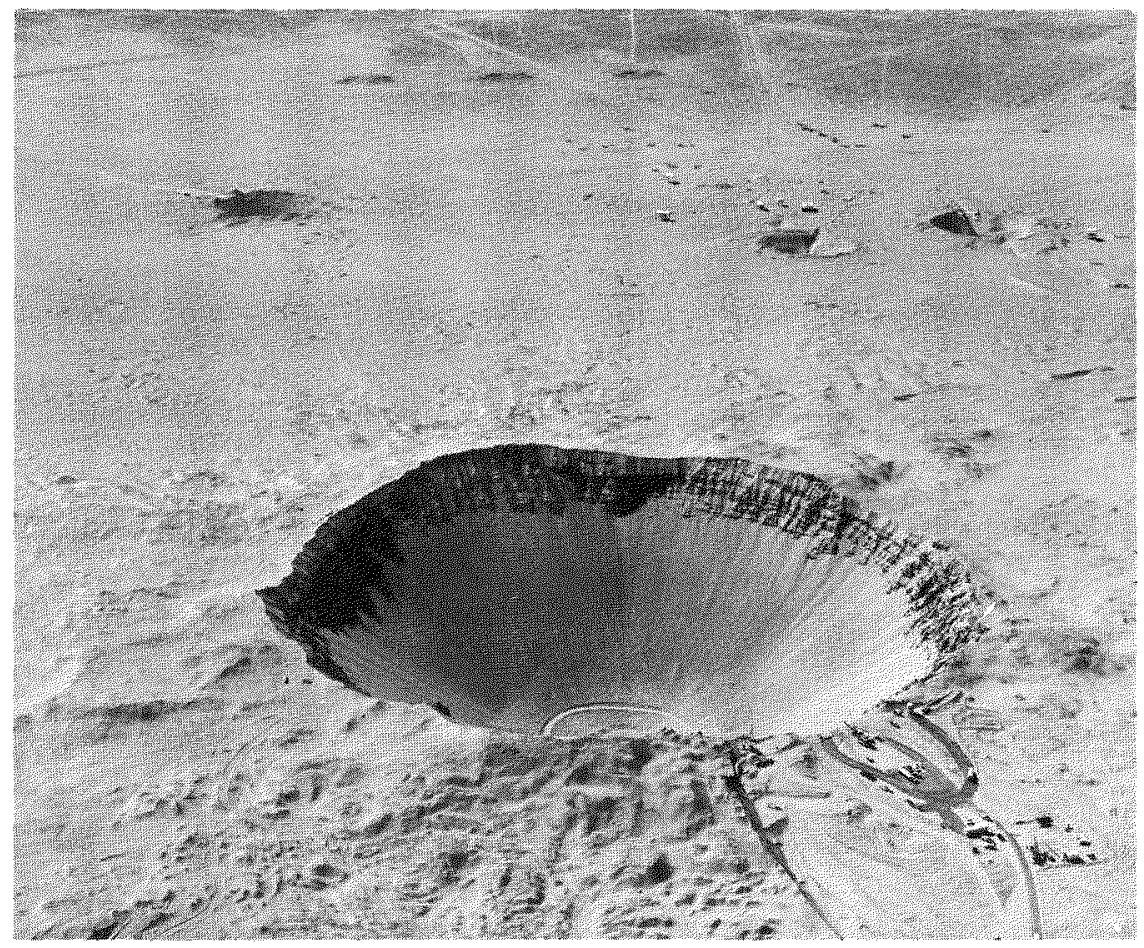

Fig. A.3 The Sedan crater.

\section{Main Dust Cloud and Base Surge}

When the fallback descends to the ground, it entrains air and fine dust particles which are carried downward. The dust-laden air upon reaching the ground then moves outward as a result of its momentum and density, thereby producing an unusual type of low-lying dust cloud called a "base surge." In the course of time, the base surge spreads out and gradually disperses.

Regardless of the mechanism, throwout crater formation is apparently always accompanied by a base surge cloud. If the detonation medium is one in which gas acceleration plays a role, however, a cloud consisting of particulate matter and the hot gases escaping from the cavity may also form and rise to a height of thousands of feet. This is generally referred to as the "main cloud," to distinguish it from the associated base surge cloud. The latter surrounds the base of the main cloud and spreads out initially to a 
greater distance. The main cloud and base surge formed in the Sedan explosion are seen in the photograph in Fig. A.4 taken six minutes after detonation time. In the Danny Boy event, on the other hand, in which gas acceleration was insignificant, there was no main cloud but only a base surge cloud, as shown in Fig. A.5. In the Cabriolet experiment, a small main cloud formed in addition to the base surge.

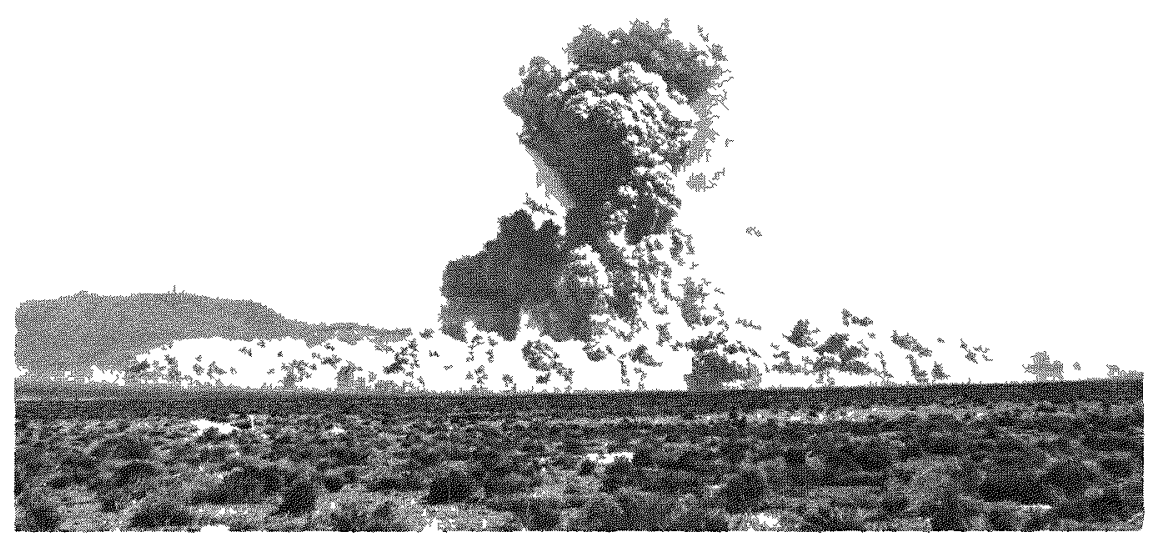

Fig. A.4 Main cloud and base surge from the Sedan test.

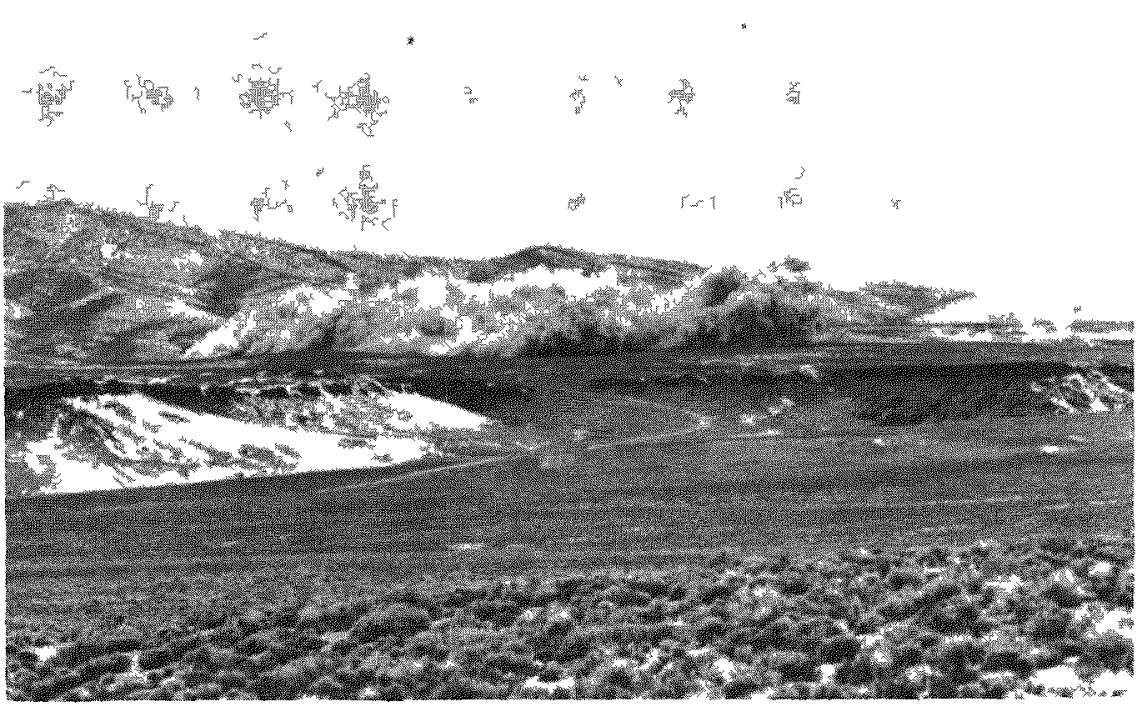

Fig. A.5 Base surge cloud from the Danny Boy test. 


\section{Early and Delayed Fallout}

The particles in the main cloud and base surge eventually fall to earth over a period of time; these particles constitute what is called the "fallout." The larger particles in the clouds descend in the first 24 hours or so after the explosion and are found mostly just downwind from the detonation site. This represents the "early" or "local" fallout. The very small particles may remain suspended in the air for considerably longer times before they reach the ground as "delayed" fallout. By the time the particles have descended to earth, mainly with rain or snow, they may have traveled great distances and are consequently spread widely, although in extremely small amounts, over large areas.

Because the base surge does not rise as high as the main cloud (see Fig. A.4), the fallout particles from the former reach the ground sooner. Most of the contribution of the base surge to the fallout generally occurs within a short distance of the explosion site. Thus, in the Danny Boy test, when essentially all the fallout came from the base surge cloud, the major part was found within a distance of 2 miles from the crater.

The importance of the fallout from the safety standpoint is that it carries some of the radioactive debris of the nuclear explosion. The major proportion of the radioactivity produced is carried down by the fallback which descends into the initial crater and in its immediate vicinity. Some radioactivity, however, remains with the particles in the main cloud and the base surge. Hence, the fallout also contains a fraction of the radioactive material. The distribution of the early fallout, in particular, and the associated radioactivity have therefore been studied extensively in connection with nuclear excavation projects. These studies, made in the interest of public safety, are described in later sections of this Appendix.

\section{Formation and Reflection of Blast Wave}

In addition to the shock wave in the ground, which is somewhat weaker than for a contained explosion of the same energy yield (Chapter 3), a nuclear excavation explosion is accompanied by the production of a shock wave in the air; this is usually referred to as a "blast wave." The strength (or overpressure) of the blast wave depends on the energy yield of the explosion, the depth of the 
detonation point, the nature of the underground rock, and the existing weather conditions. The smaller the yield and the greater the depth of burial, the weaker will be the air blast wave.

The air blast is first mainly directed upward and it then expands outward. There are various atmospheric conditions, however, which can cause the blast waves moving in different directions to be bent back and, at the same time, focused so that they strike the earth at distances of 50 to 150 miles (or more) from the point of origin. In between the areas where the blast wave is focused, there is essentially no overpressure (i.e., no pressure in excess of the ambient atmospheric pressure) at ground level and no significant noise. At the focal points, a small overpressure can be detected and the noise of the blast wave can be heard.

Even a fairly small overpressure can sometimes cause broken windows and cracked plaster. However, the meteorological phenomena and other atmospheric conditions that result in blast waves being bent back to earth and focused are fairly well understood. Nuclear excavation explosions would therefore be conducted only when the circumstances were such that focusing into populated areas, with the attendant probability of minor damage, would not occur.

\section{PREDICTION OF RADIATION EXPOSURES}

\section{Introduction}

In a nuclear excavation, a certain amount of local fallout cannot be avoided. The potential hazard can be reduced, however, by keeping the radioactivity of the fallout as low as practicable. Consequently, as an aspect of the safety program related to nuclear excavations, special "clean" explosives have been designed that produce minimal quantities of biologically important radioisotopes. The efficacy of these designs has been confirmed by experimental tests.

Since a fission explosion of a given energy yield leaves more radioactive residues of biological concern than does a fusion explosion of the same energy yield (p. 30), the explosives for nuclear excavations are designed to derive most of their energy from fusion. The amount of neutron-induced radioactivity from fusion is greater 
than from fission, but the materials used in the construction of the device are selected so as to avoid the production of biologically important radioisotopes as a result of neutron absorption. The neutron-induced radioactivity can be decreased to a large extent by the use of a neutron-absorbing shield, but even without a shield the amount of radioactivity of concern to man is much less than if the explosive energy were derived solely (or mainly) from fission.

In planning a nuclear excavation project, calculations are made to determine the wind conditions that will permit the operation to be conducted safely from the radiological standpoint. A mathematical method has been developed which yields accurate predictions of the areal distribution of the radiation intensity, but it requires the use of a computer. This method will be described on page 244 . But first, consideration will be given to a simpler approach. It is less exact than the mathematical model and provides more limited information, but it is useful for preliminary planning and for operational purposes.

\section{The Fallout Sector}

The (downwind) fallout sector is the area on the ground where local fallout may be expected (p. 150). Before a nuclear excavation event is conducted, there must be assurance that the fallout sector will not include any area where exposure from the radioactivity in the fallout would reach the appropriate radiation protection standards (Chapter 8). The fallout sector depends on the wind speeds and directions up to the top of the dust cloud (i.e., main cloud or base surge). Consequently, prediction of fallout sectors allows specification of the wind patterns that would lead to the desired conditions. The excavation would be carried out only if these conditions were met.

In order to illustrate a simple method for deriving fallout sectors, a particular wind pattern will be assumed. Suppose the circumstances are such that the dust cloud is expected to rise to an altitude of 5000 feet above the surface. On the basis of calculations and previous experience, satisfactory predictions can be made of the height attained by the cloud when it becomes stabilized, that is, before it starts to spread out by mixing with the surrounding atmosphere. An assumed pattern of wind speeds and directions at intervals of 1000 feet up to a height of 5000 feet above the explosion site is given in the accompanying table. 
Wind Data for Fallout Sector Estimate

\begin{tabular}{ccccc}
\hline $\begin{array}{c}\text { Height above } \\
\text { surface (feet) }\end{array}$ & $\begin{array}{c}\text { Direction } \\
\text { (degrees) }\end{array}$ & $\begin{array}{c}\text { Speed (miles } \\
\text { per hour) }\end{array}$ & $\begin{array}{c}\text { Weighting } \\
\text { factor }\end{array}$ & $\begin{array}{c}\text { Vector } \\
\text { in Fig. A.6 }\end{array}$ \\
\hline 0 & 150 & 10 & 0.5 & OA \\
1000 & 180 & 10 & 1.0 & AB \\
2000 & 200 & 15 & 1.0 & BC \\
3000 & 220 & 20 & 1.0 & CD \\
4000 & 230 & 20 & 1.0 & DE \\
5000 & 240 & 24 & 0.5 & EF \\
\hline
\end{tabular}

The wind speed indicated at each level in the table, with the exception of that at the surface and at 5000 feet, is assumed to represent the (constant) wind speed through a 1000-foot layer centered at each level; that is, from 500 to 1500 feet, from 1500 to 2500 feet, from 2500 to 3500 feet, and from 3500 to 4500 feet. The surface and 5000-feet values are representative of wind speeds in the 500 -foot layers from the surface to 500 feet, and from 4500 to 5000 feet, respectively. The "weighting factor" in the fourth column refers to the relative thickness of the layer to which each particular wind speed is applied; a layer 1000 feet thick has a weighting factor of 1.0. The wind speed multiplied by the weighting factor gives the weighted wind speed for each air layer (i.e., the wind speed weighted by the thickness of the layer).

Starting at the point $O$ in Fig. A.6, representing the surface just above the explosion point, a line OA is drawn for a wind direction of $150^{\circ}$ and length proportional to the weighted wind speed, i.e., $10 \times 0.5=5$ (miles per hour). This line is called the "weighted wind vector." It represents (approximately) the direction of movement and weighted speed of the dust cloud from the surface to a level of 500 feet. The vector $\mathrm{AB}$, for a wind direction of $180^{\circ}$ and length proportional to $10 \times 1.0$ (miles per hour), is drawn to represent the weighted wind vector from 500 to 1500 feet. Similarly, the lines BC, $\mathrm{CD}, \mathrm{DE}$, and $\mathrm{EF}$ give the weighted wind vectors to the top of the cloud at 5000 feet above the surface.

*A vector has both magnitude and direction; in this case the magnitude of the vector is the weighted wind speed and the direction is that of the wind in a given air layer. 


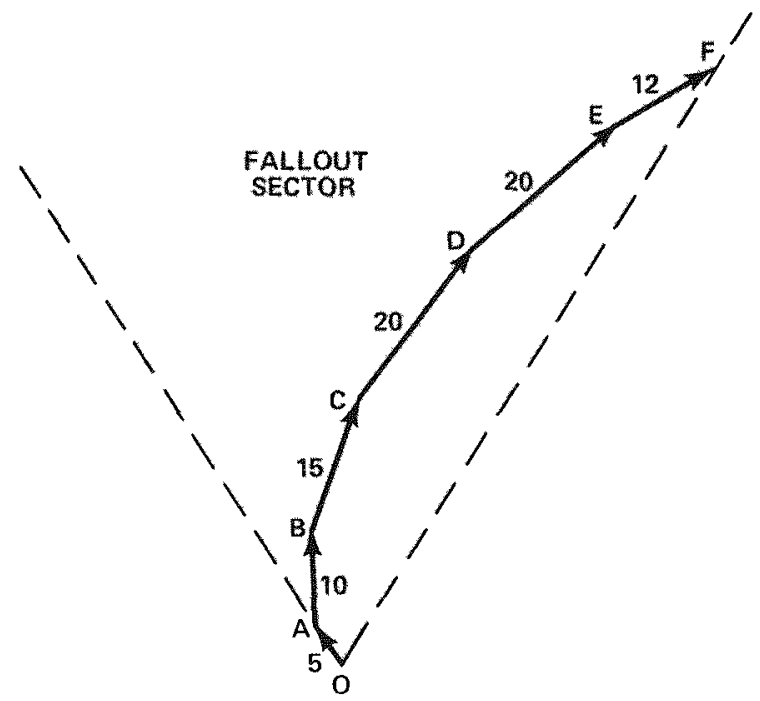

Fig. A.6 Prediction of downwind fallout sector (hypothetical).

The downwind fallout sector, where fallout may be expected on the ground, is then the shaded area enclosed by continuations of the lines OA and OF. Particles in the cloud of debris that have only reached a height between the surface and 500 feet, will be carried along the direction OA. They will thus gradually fall to the surface more or less along that line. At the other extreme, particles that have been carried to the top of the cloud will initially be transported by the wind at this level along the direction EF. Subsequently, as the particles descend, they will be affected by winds at successively lower levels and they will eventually reach the ground along the direction of the line OF. Hence, all the particles will be expected to descend to the ground in the directions between $\mathrm{OA}$ and $\mathrm{OF}$.

The shaded area in Fig. A.6 thus provides a general indication of the region in which particles may be expected to fall out from the dust cloud produced in an underground nuclear detonation. It should be pointed out that, in deriving this area, the basic assumption has been made that the wind speeds and directions remain unchanged over the whole distance traveled by the particles. Conditions are rarely steady, however, and the fallout sector at greater distances from the explosion point can be adjusted to allow for changes in the wind. Since several hours will elapse before the fallout reaches such 
distant areas, there is time to modify plans for protective actions should any changes be required.

The fallout sector is the area over which particles may descend to the ground. The important quantity, however, is the total radiation exposure that could result at various locations in the fallout area. For this purpose it is necessary to predict the radiation intensities (or exposure rates), that is to say, the radiation exposures per unit time. The total radiation exposure that may be expected during a specified period at a given location can then be calculated from the radiation intensities (see footnote, p. 167). The stages in the prediction of radiation exposures are described in the following sections.

\section{Radioactivity in Fallout}

In order to calculate the expected radiation intensities in the local fallout area, it is first necessary to know how much radioactivity is released to the atmosphere and what fraction of this is deposited as local fallout. The radioactivity released depends primarily on the total quantity of radioactivity produced, on the scaled depth of burial (p.54), and on the nature and degree of water saturation of the rock in which the explosion occurs.

The radioactivity produced by an underground explosion arises partly from fission products and partly from induced activity resulting from neutron absorption. The radioactivity from fission products can be calculated from the expected fission energy yield of the nuclear explosive used. The quantity of induced radioactivity depends on the fission and fusion energy yields, on the design and materials present in the nuclear explosive, on the nature of the rock being excavated, and on the possible use of a neutron shield. In spite of the complexity of the problem, reliable calculations can be made of the quantities of radioisotopes produced by neutron activation. For all practical purposes, the computations can be limited to some five important isotopes which contribute about 95 percent of the potential radiation exposure from the induced activity.

As a result of experience and theoretical studies, which are checked by data from actual tests, designers of nuclear explosives are able to predict fission and fusion energy yields quite accurately. For calculating the expected radioactivity release in an excavation operation, a design yield and a maximum credible yield are provided. In order to adopt a conservative approach, and tend to overestimate 
the probable consequences, the maximum credible yield is used in the calculations.

Next, the scaled depth of burial (i.e., the actual depth of burial in feet divided by the cube root of the energy yield in kilotons TNT equivalent) will be considered. If the nuclear explosive is at (or close to) the earth's surface, so that the depth of burial is essentially zero, a large proportion of the radioactive residues will enter the atmosphere. On the other hand, if the scaled depth of burial is great (e.g., more than about 350 feet), the radioactivity will generally be contained in the ground. Between these two extremes, the fraction of the total radioactivity that escapes from a detonation in a given rock (and water content) will depend on the scaled depth of burial of the nuclear explosive. By using data obtained from past nuclear excavation experiments, Fig. A.7 has been prepared. It shows the percentage of the radioactive material that is deposited as local fallout for explosions with scaled depths of burial up to about 250 feet.

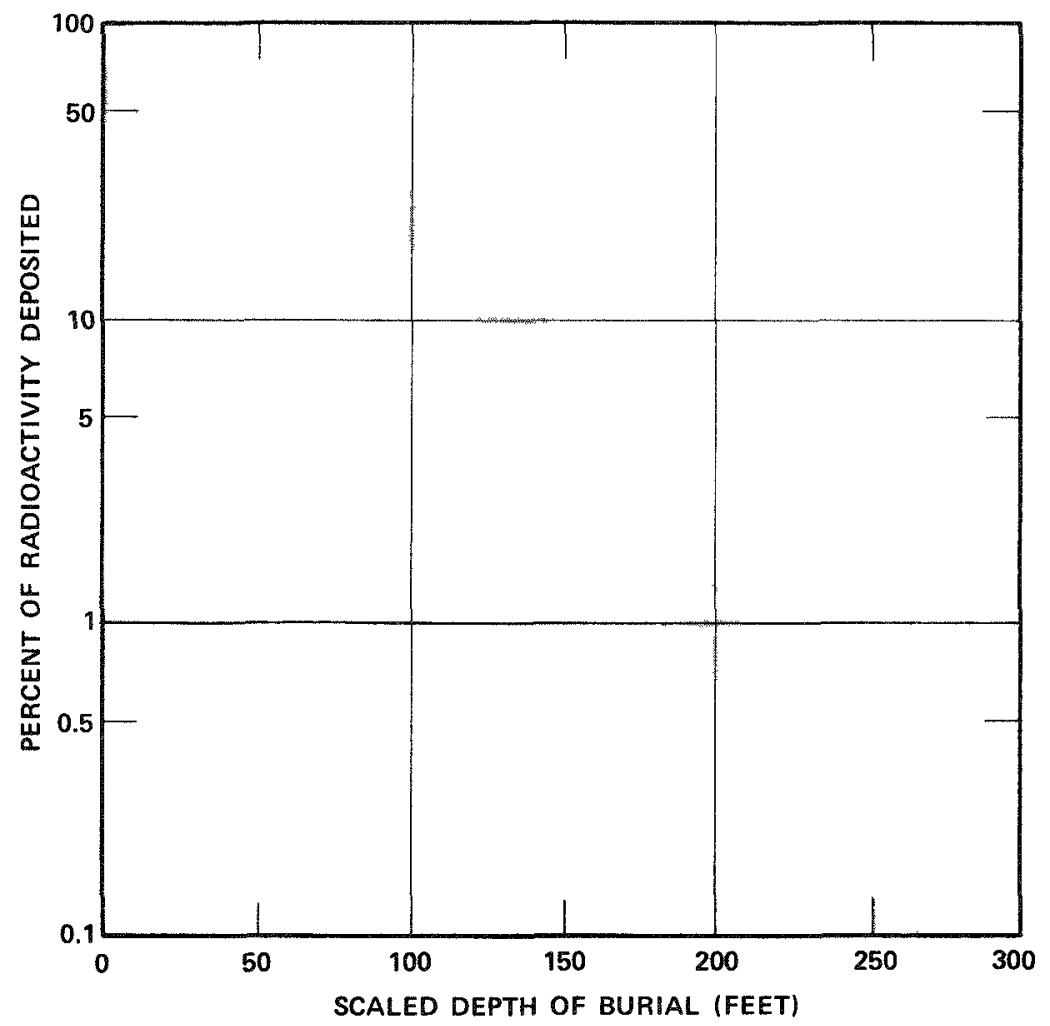

Fig. A.7 Percentage of radioactivity deposited as local fallout in cratering tests. 
It is seen that the relationship between the fallout fraction (or percentage) and scaled depth of burial is not a single line, but a fairly broad band. This means that, for a given scaled depth of burial, the fraction of the total radioactivity appearing as local fallout is not a precise quantity. The reason is that this fraction depends to some extent on the nature of the rock being excavated and, particularly, on the degree of water saturation. If possible, data from explosions in the same rock type are used to define the fallout percentage more exactly. But if such data are not available, then the maximum value from Fig. A.7 would be used for predicting the local fallout activity.

The total radioactivity of the explosion residues is now multiplied by the maximum fallout fraction for the known scaled depth of burial. The result gives the maximum amount of radioactivity that is expected to appear in the local fallout from the given excavation experiment. Since maximum yield and maximum fallout fraction are used at this stage of the calculation, the subsequent estimates of fallout radiation intensities include a substantial safety factor.

\section{Radiation Intensity from Fallout}

Two procedures are commonly used for predicting the downwind pattern of radiation intensities from the fallout in a nuclear excavation operation. The more accurate method, which requires the use of a computer, is outlined in the next section. Another approach, described below, satisfies the requirements of an operationally useful method for predicting radiation intensities that is both rapid and simple. It does not give a complete pattern of radiation intensities over the local fallout area, as does the other method, but only the approximate intensities along a downwind center line of the fallout sector where the values are expected to be maximal (Fig. A.8).

The simpler method involves an analog (or scaling) technique in which a prediction for a planned excavation explosion is based on observations made at previous events. The characteristics (e.g., scaled depth of burial) of the events chosen for comparison must be similar to those expected for the proposed detonation. The basic assumption of the analog technique is that the essential fallout pattern is determined mainly by the state of the atmosphere at the time of the underground nuclear detonation. The details, however, are modified in a relatively straightforward manner by the wind speed and direction, by the height attained by the radioactive cloud, and by the 


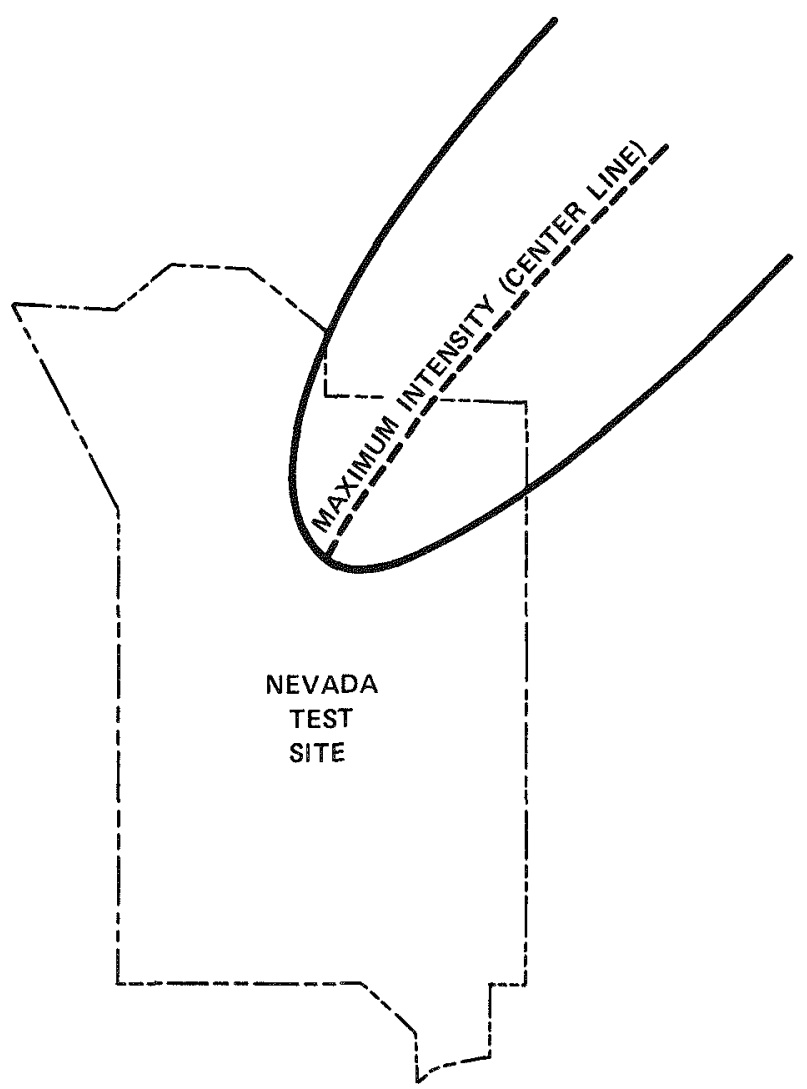

Fig. A.8 Predicted direction of maximum fallout radioactivity from a cratering test (hypothetical).

total radioactivity of the fallout. Thus, by comparing the expected values for these quantities for a planned detonation with those previously observed in a similar situation, the center-line radiation intensities can be predicted for various distances from the site of the explosion.

The procedure described above depends, of course, on the availability of data from previous nuclear excavation tests. In all such experiments, performed at the Nevada Test Site, measurements have been made of the radiation intensities from fallout over a large downwind area. By reducing the results to standard conditions, the external radiation intensities (i.e., from gamma rays) observed on the ground can be represented approximately by a single curve, as in Fig. A.9. In practice, the figure would show the actual exposure rate 


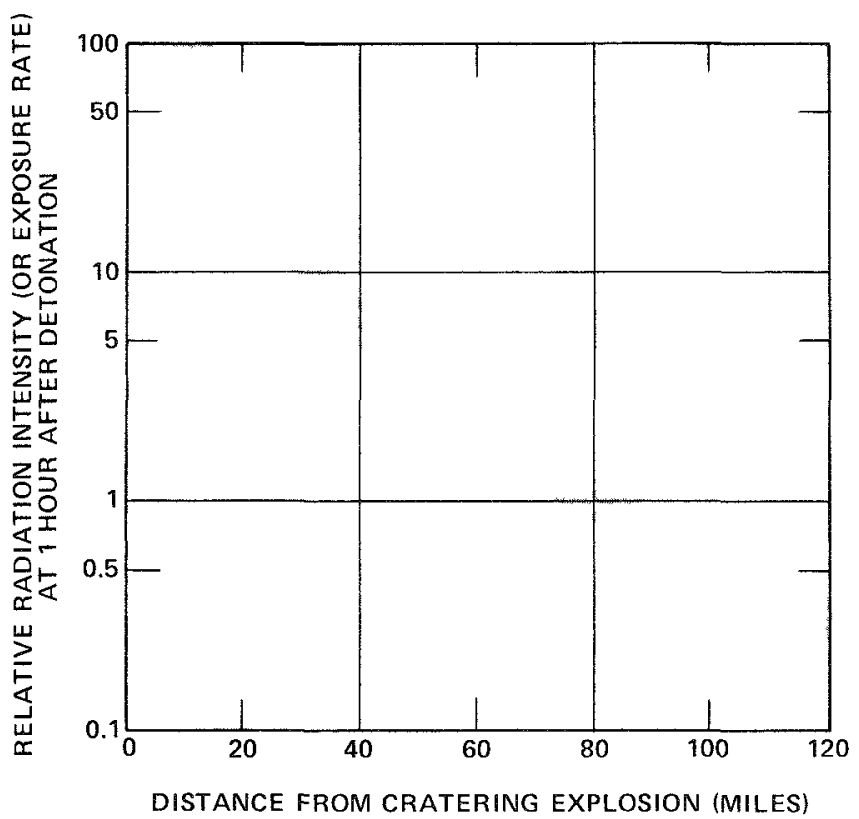

Fig. A.9 Radiation intensity (or exposure rate) at increasing distances from a cratering test.

at 1 hour after the detonation, expressed in roentgens (or milliroentgens) per hour, rather than relative values.

By means of the curve, approximate center-line radiation intensities (or exposure rates) can be predicted from the expected conditions for the excavation explosion. The predictions are not expected to be exact, as is implied by the width of the curve, since they do not yet allow for all possible variable factors (e.g., the nature and moisture content of the rock in which the device is exploded) and the detailed atmospheric conditions. Thus, the single curve in Fig. A.9 for all situations is somewhat of an oversimplification. Nevertheless, the scaling (or comparison) technique has been found to provide a useful indication of the expected center-line radiation intensities from fallout in excavation experiments with nuclear explosives.

A matter of special interest with regard to Fig. A.9 is that the expected radiation intensity decreases with increasing distance from the explosion point. Thus, at 120 miles away the expected intensity might be only about 1 percent of that at 20 miles. If the dust cloud 
should pass over an inhabited area at some distance downwind from the explosion site, the radiation level will then be quite low.

The predictions based on the scaling procedure do not take into account the possibility of the formation of hot spots as a result of precipitation (p.145). This can be included, however, in the more detailed fallout model described in the next section.

\section{Fallout Radiation Model}

The accurate procedure for predicting fallout radiation patterns is more fundamental than the scaling technique just described. It can be applied either to the fallout from the main cloud or from the base surge (or both). The basic quantities required for the calculations are itemized below and the methods whereby they are obtained are outlined.

(a) The heights of the top and bottom of the stabilized main cloud (or base surge). By the use of relationships derived from previous experience, the required heights can be predicted from the total energy yield of the explosion, the depth of burial of the explosive, and the nature (and moisture content) of the rock.

(b) The amount of radioactivity that is expected in the local fallout. This is obtained in the manner described above (p. 239). As already noted, there is some uncertainty in the fraction of the total radioactivity that appears in the local fallout. Studies are in progress for developing a mathematical model that will reduce the uncertainty.

(c) The variations of the radioactivity with the size of the particles in the cloud. In general, a larger particle is expected to carry more radioactivity than a smaller one. The distribution of activity with particle size is obtained from the best mathematical fit to the observations at previous nuclear excavations in the same rock type as that for which the predictions are being made.

(d) The rate of fall of particles in the cloud. The rate of fall depends on the size of the particle and to a minor extent on the height from which it falls. Rates of fall of particles of different sizes, having a given density, under the influence of gravity and against the resistance of the air can be calculated by established methods. While descending from the cloud, the rate of fall of a particle may occasionally be affected by the vertical component of the wind.

(e) The wind pattern over the area of the expected fallout. The wind speeds and directions at various altitudes determine the 
transport of the cloud, that is, the direction and rate at which the cloud moves, and also its dimensions, as a result of turbulent mixing with the atmosphere. If the wind data at the time and place of the explosion are used to predict the fallout pattern, location errors may result from changes in both time and place. Progress is being made in the development of a wind prediction model for following the transport of the cloud particles during their fall to earth. Calculation of the deformation of the cloud that results in an increase in its dimensions is based on the theory of eddy (or turbulent) diffusion such as is commonly used in atmospheric studies by meteorologists and others.

In the model for predicting the gamma-ray (external) exposure rate from fallout, utilizing the foregoing information, the initial cloud is considered to be comprised of eleven individual clouds, each containing particles of uniform size. Each cloud in the model is then divided into eleven horizontal disks, from bottom to top. There are thus 121 disks, in each of which the particles have the same rate of fall and are affected by essentially the same wind speed and direction. As it is transported downwind, each disk increases in size as a result of eddy diffusion into the surrounding air. A computer code is then used to determine when and where the particles of different sizes reach the ground as fallout. From the total expected radioactivity of the fallout and the distribution of activity with particle size, the external exposure rate on the ground can be computed.

By following the motion of only the center of each disk, where the radioactivity is at its highest, the center-line (or maximum) radiation intensity is obtained as a function of distance from the explosion site in a nuclear excavation explosion. The curve is similar to that in Fig. A.9, except that it gives the actual exposure rates. The predicted curves obtained in this manner have been found to be in good agreement with those observed in several excavation tests.

If the whole disk is included in the calculation, the final result gives the distribution of the external radiation exposure rates over the whole of the significant fallout area. The data are usually plotted in the form of a series of contour lines of equal exposure rates at 1 hour after the detonation (see Fig. A.9). From these rates the total (accumulated) exposure over a period of time can be calculated.

There are two main sources of uncertainty in the computations outlined above. They are concerned with the possibility of the formation of hot spots if the cloud encounters a region of 
precipitation and the difficulty of predicting the long-range cloud trajectory. Fortunately, these uncertainties are usually significant only at considerable distances from the excavation site. These areas are not reached for several hours after the detonation by which time the radioactivity of the cloud would be greatly decreased.

The effect of precipitation on the radiation distribution on the ground could be included in the mathematical model if the location, intensity, and duration of the precipitation were known. Although general precipitation patterns may be predictable, the details are uncertain, especially at long distances from the explosion site. In an actual operation, however, if the cloud were tracked at different levels and if radar information provided data concerning rain cells, it should be possible to identify hot spot locations and to estimate the radiation intensity with sufficient detail to be of practical use.

If the actual cloud trajectory differed from the predicted one used in the calculations, the radiation intensities at various distances from the excavation site would not be greatly in error. However, the geographical locations would not be the same as those expected. As already stated, it is probable that uncertainties in the cloud trajectory will be decreased as a result of the development of a better wind prediction model.

\section{Prediction of Total External Radiation Exposure}

It will be observed that the radiation intensities in Fig. A.9 refer to "1 hour after detonation," often represented in the abbreviated form of " $\mathrm{H}+1$ hours." The radioactive cloud may travel only a short way-perhaps 10 or 20 miles-within an hour after the detonation time, so that at greater distances there would be no actual fallout at that time. The hypothetical $\mathrm{H}+1$ hour radiation intensity is then a measure of the radioactivity that the fallout, when it does arrive at a particular location, would have had 1 hour after the detonation, that is, while it was still in transit.

The importance of the hypothetical $\mathrm{H}+1$ hour radiation intensity is that it can be used for calculating the total external radiation exposure that may be expected from fission products in fallout. It is for this reason that, when discussing the rate of decay of fission products in Chapter 2, the radioactivity was taken to be 100 units, as the reference value, at 1 hour after the explosion. The radioactivities at later times were then expressed in relation to this reference value. 
The total external exposure from fission products during a specific time period may be obtained from the area for that time period under a curve such as Fig. 2.17. In practice, however, it is preferable to express the time variation of the fission product activity by means of a simple equation from which the total radiation exposure can be obtained mathematically (by integration).

To the contribution from fission products there must be added the exposure arising from neutron-induced radioactivity. As seen on page 239 , the quantities of the most important radioisotopes produced in this manner can be predicted quite accurately. The associated radiation exposure rates and total exposures can then be computed from the known half-lives and the characteristics of the gamma radiation emitted. These are, in fact, included in the mathematical fallout model.

\section{Radiation from the Cloud}

The predicted radiation intensities and exposures considered so far are those that might result from fallout particles deposited on the ground. If the dust cloud does not rise very high, there may be some exposure to radiation on the ground directly from the cloud itself. This would result from the presence of radioactive isotopes that emit gamma rays. These radiations can penetrate to considerable distances in the air, although the intensity decreases quite rapidly with increasing distance from the source of the gamma rays (p. 14).

An idealized curve showing the variation of the radiation intensity on the ground with time during and after the passage of a cloud is given in Fig. A.10. It applies to a hypothetical case in which the maximum intensity is 10,000 times the background intensity of natural radiation prior to the arrival of the cloud. When the cloud approaches a given point, the radiation intensity on the ground starts to increase rapidly, reaching a maximum when the cloud is directly over the point (i.e., at 10 minutes in the case depicted in Fig. A.10). Then the cloud moves away and the radiation intensity falls off sharply.

During the passage of the cloud, some of the radioactivity is deposited on the surface as fallout. The radiation intensity on the ground after the cloud has moved away is then due to the fallout only. The radioactivity subsequently decreases with time in the usual way. 


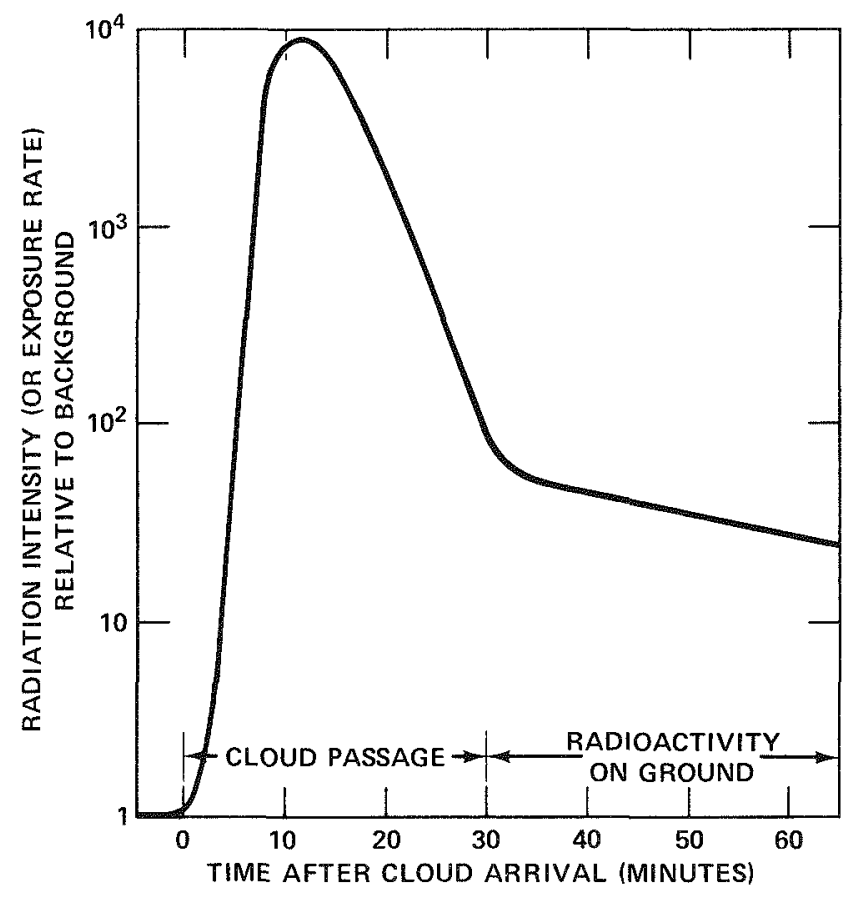

Fig. A.10 Radiation intensity on the ground as a result of cloud passage (hypothetical).

To obtain the total amount of external radiation exposure at a point on the ground, the amount contributed by the cloud during its passage must be added to that from fallout. The former is obtained from the area under the curve in Fig. A.10, after allowing for the radiation from fallout remaining after cloud passage. The fraction of the external exposure received from the cloud varies with the circumstances. If the cloud is fairly low and contains a large quantity of gamma-emitting isotopes, the fraction will be larger than from a high cloud. In the latter case absorption of part of the gammaradiation energy by the atmosphere and attenuation by distance will result in a substantial decrease in the radiation intensity on the ground.

\section{Long-Range Cloud Studies}

For the smaller particles in the dust cloud formed in a nuclear excavation explosion, the up and down transport by air motion is 
more important than the fall under gravity. Consequently, most of these small particles remain suspended in the air. However, the cloud dimensions continually increase-upward, downward, and sideways-as a result of turbulent diffusion. The clouds can thus become very large and travel great distances as the particles are transported by the wind. The small particles ultimately form the delayed fallout (p.234).

There is little or no actual fallout from these large clouds, but in the course of a few days they may expand so that their lower portions touch the earth's surface. Some of the particles may then be removed by impact with the ground and with vegetation. There is also the possibility that some particles will be brought to the ground by rain. Consideration must therefore be given to these long-range clouds, especially within the first three or four days after the excavation event, when they may have traveled distances of several hundred miles.

A diffusion model has been developed, with meteorological data (including precipitation conditions) as the input, which permits calculation of the concentrations of individual radioisotopes in the long-range cloud as a function of time (and distance). The model also provides the capability of predicting the amounts of such biologically important isotopes as iodine-131 deposited on the ground over which the cloud is expected to pass.

\section{Radiation Protection}

The calculations described in the preceding sections would be used to determine that the conditions are such that exposure from the radioactivity in the fallout will be less than the appropriate radiation protection standards. Only in this case would the proposed nuclear excavation event be conducted. The cloud (and base surge) formed would be tracked by aircraft and by tetroons, as explained in Chapter 7. The observations would indicate if any changes in the planned protective actions are desirable.

The radiological safety program described in Chapter 8 would be operated on the ground. As a general rule, additional radiation dosimeters would be issued to a number of individuals living downwind from the excavation site. In the environmental surveillance aspects of the program, special attention would be paid to iodine-131 and tritium in milk and water supplies. 


\section{Radioactive Isotopes in Surface Water}

The possibility of entry of radioactivity from a nuclear excavation explosion into groundwater is similar to that for a contained explosion. As seen in Chapter 4, there is essentially no danger that useable water supplies, originating from groundwater, will be contaminated. Some attention must be paid, however, to the effects of a nuclear excavation on surface waters.

There are two ways in which fallout could cause radioactivity to be present in surface waters: (1) by the particles falling directly onto open water surfaces, such as streams and lakes, and (2) by gradual transfer of the particles from the land surface into the water. The first type of potential contamination would occur within a few hours of the detonation while the fallout is descending. The water affected during this time would generally move on downstream. The second kind of contamination would continue, although usually at a very low level, for some time after the explosion.

If the fallout particles enter the water directly, some of the radioactive material will be dissolved by the water and some will remain as suspended particulate matter. As the water flows on, much of the dissolved radioactivity would be removed by sorption on soil, rock, and other solid matter (p. 78). As with groundwater, dispersion and natural decay would also tend to decrease the concentrations of radioisotopes. If adequate information is available concerning the flow rate of the water and the chemical and physical characteristics of the stream beds, prediction of the migration of individual isotopes could be made along the lines indicated in Chapter 4 for groundwater.

If fallout particles are transported from land surfaces into surface water by runoff from rainfall or other precipitation, prediction of the potential contamination requires, in the first place, that the drainage basins in the area of the nuclear excavation be delineated. Then, the expected areal distribution of the fallout activity must be available. Something must also be known about the attachment of fallout particles to the ground surface on which they have descended and their removal from this surface by rain, snow, and possibly wind. Predictions of the potential contamination are thus quite difficult. As a general rule, however, it is expected to be less significant than from the direct entry of fallout particles into surface waters.

In addition to calculations of possible radioactive contamination of surface waters by fallout, field tests have been performed with 
actual fallout material and also with known amounts of radioactive isotopes of cesium, iodine, and strontium. These tests are carried out by the U.S. Geological Survey and the results are used to improve the calculational procedures.

In the United States, the Nevada Test Site is the only region where nuclear excavation experiments have been conducted so far. In this area there are no permanent streams or bodies of water and the annual precipitation is small.* The contamination of surface waters has thus not been a problem. At other locations, appropriate steps would be taken for the protection of any useable surface water supplies that might be in the vicinity of the excavation site. In any event, mixing and dilution of water and other factors, such as sorption and natural decay, would tend to decrease the concentration of radioisotopes. The radioactivity of surface waters entering the ground would be rapidly reduced by sorption and dispersion.

\section{Bioenvironmental Effects}

Radioisotopes falling on the ground or entering surface waters could have various effects on the environment and on food chains that lead to man. These subjects have been included in the discussion of the bioenvironmental program in Chapter 9. Adequate precautions are taken in connection with nuclear cratering projects to avoid hazards to man, to animals, and to the environment. The only area significantly affected would be that close to the excavation site.

\section{SUMMARY}

For excavation applications, a nuclear explosive is detonated at an appropriate depth. The cavity formed by the explosion breaks through the surface, leaving an excavation crater. The breakthrough is accompanied by a shock (or blast) wave in the air.

Most of the radioactivity produced by the nuclear detonation remains in the material that falls back immediately into the initially formed crater and onto the surrounding lip. Some of the radio-

\footnotetext{
* Lake Mead, at a distance of more than 100 miles, is too far away to be appreciably affected by fallout from experiments at the Nevada Test Site.
} 
activity, however, is in the dust clouds (i.e., the base surge and main cloud) that may form. The main cloud may rise to a height of many thousand feet, and the larger particles present descend to the ground as local fallout, chiefly in the downwind direction. Special nuclear explosives have been developed that produce less radioactivity of concern to man than from an explosion of the same energy yield derived from fission alone.

Methods have been developed for predicting the area that might be affected by fallout and for calculating the radiation exposures that might be expected in that area at various distances from the explosion point. A nuclear excavation is conducted only if there is assurance that exposure from the radioactivity present in the fallout will be within the limits of the appropriate radiation protection guidelines. The radiological program following the detonation would indicate if any changes are desirable in the protective actions planned before the event.

Fallout particles can enter surface waters, either directly or indirectly. So far, nuclear excavation explosions have been performed in the United States only at the Nevada Test Site. Because the rainfall is small and there are no permanent streams in the area, contamination of surface waters has not been a problem. If nuclear excavations are conducted at other sites, precautions would be taken to assure the safety of water supplies. 


\section{REFERENCES}

A Report on Underground Nuclear Testing, U. S. Atomic Energy Commission, September 1969, TID-25180.

A Selected Bibliography of the Civil, Industrial, and Scientific Uses for Nuclear Explosives, U.S. Atomic Energy Commission, TID-3522 and Supplements. Bioenvironmental Safety Studies, Amchitka Island, Progress Report for Fiscal Year 1968, Battelle Memorial Institute, BMI-171-116.

Environmental Statement: Cannikin, U. S. Atomic Energy Commission, 1971.

Environmental Statement: Underground Nuclear Test Programs, Nevada Test

Site, Fiscal Year 1971, U. S. Atomic Energy Commission, November 1970.

Fuller, R. G., Amchitka Biological Information Summary, Battelle Memorial Institute, 1969.

Glasstone, S. (Editor), The Effects of Nuclear Weapons, U.S. Government Printing Office, 1962.

Holzer, A., Plowshare and the Environment, University of California Radiation Laboratory, November 1970, UCRL-72830.

Knox, J. B., et al., Radioactivity Released from Underground Nuclear Detonations: Source, Transport, Diffusion, and Deposition, University of California Radiation Laboratory, March 1970, UCRL-50230(Rev. 1).

Merritt, M. L., Physical and Biological Effects: Milrow Event, U.S. Atomic Energy Commission Nevada Operations Office, December 1970, NVO-79.

Nevada Test Site: Studies of Geology and Hydrology, The Geological Society of America, Inc., Memoir 110, 1968.

Proceedings of the Symposium on Engineering with Nuclear Explosives, Las Vegas, Nevada, January 1970, CONF-700101, Vols. 1 and 2.

Proceedings for the Symposium on Public Health Aspects of Peaceful Uses of

Nuclear Explosives, Las Vegas, Nevada, April 1969, U. S. Department of Health, Education and Welfare, SWRHL-82.

Project Rulison: Post-Shot Plans and Evaluations, U.S. Atomic Energy Commission Nevada Operations Office, December 1969, NVO-61. 
Reports Currently Available in Plowshare Open File, U. S. Atomic Energy Commission Nevada Operations Office.

Schultz, V., References on Nevada Test Site Biological Research. Great Basin Naturalist, XXVI, Dec. 31, 1966, Nos. 3-4, p. 79.

Special Papers on Underground Nuclear Explosions at the Nevada Test Site. Bulletin of the Seismological Society of America, Vol. 59, No. 6, December 1969 , pp. 2167-2382.

Technical Discussions of Offsite Safety Programs for Underground Nuclear Detonations, U.S. Atomic Energy Commission Nevada Operations Office, May 1969, NVO-40 (Rev. 2). 


\section{GLOSSARY}

AFTERSHOCKS Minor seismic tremors that may follow an underground nuclear detonation. (Also the secondary tremors following the main shock of an earthquake.)

ALLUVIUM Compacted particles of rock, sand, clay, etc., deposited from water over long periods of time. Much of the earth's surface is covered with alluvium.

ALPHA PARTICLE A particle emitted spontaneously from the nuclei of some radioactive elements. It is identical with the helium nucleus, having a mass of four units (two neutrons and two protons) and an electric charge of two positive units. See Neutron, Nucleus, Proton.

AMPLITUDE In general, the maximum value of an oscillatory quantity measured from its neutral or undisturbed value in a single vibration of a series. In this book, amplitude generally refers to the displacement of a structure or of the ground.

AQUIFER An underground rock structure through which water moves.

ATOM The smallest particle of an element retaining the characteristics of that element. It has a positively charged nucleus surrounded by negative electrons with the same total charge as the nucleus. Thus, the atom is electrically neutral. See Electron, Nucleus.

ATOMIC NUMBER The number of protons (positive charges) in the nucleus. It is the same for all atomic nuclei of a given element. See Nucleus, Proton.

BACKGROUND RADIATION Nuclear (or ionizing) radiations arising from within the body and from the surroundings to which 
individuals are always exposed. The main sources of natural background radiation are potassium-40 and carbon-14 in the body; potassium-40, thorium, uranium, and their decay products (including radon gas) present in soil, rocks, and air; and cosmic rays.

BASE SURGE In this book, the low-lying dust cloud formed in an excavation explosion when the fallback descends to the ground. See Dust Cloud, Fallback.

BETA PARTICLE A charged particle of very small mass emitted spontaneously from nuclei of certain radioactive elements. Physically, beta particles are identical with electrons moving with high velocities. Most, if not all, of the primary fission products emit negatively charged beta particles. See Electron, Fission Products.

BIOENVIRONMENTAL PROGRAM See Ecology.

BIOSPHERE That portion of the earth's surface and atmosphere that can sustain living organisms.

BIOTA The animal and plant life of a region.

BLAST WAVE A compressional wave in the air, propagated from an explosion, in which the pressure rises sharply at the front. See Shock Wave.

CAVITY An expanding sphere of material vaporized by the shock wave and heat from an underground nuclear detonation; at this stage the cavity is known as the vaporization cavity. The stable cavity results when pressure in the cavity decreases and expansion stops. The radius of the stable cavity is about seven times that of the vaporization cavity.

CHIMNEY A tall, roughly cylindrical volume of broken rock and rubble formed by the collapse of the overlying medium (overburden) into the cavity produced by an underground explosion. See Cavity.

CLEAN NUCLEAR EXPLOSIVE In this book, a nuclear device which produces minimum amounts of biologically significant isotopes for a particular application in the Plowshare program.

CONTAINED EXPLOSION An explosion at such a depth underground that there should be no significant escape of radioactive material to the atmosphere.

COSMIC RAYS Radiations from space of many kinds with very high energies; a part of background radiation. 
CRATER In this book, a depression in the ground left by some underground nuclear explosions. A throwout crater results from the expulsion of ground material by the expanding gases in the explosion cavity. A subsidence crater may be formed if the collapse of the chimney material reaches the ground surface. See Cavity, Chimney.

CRITICAL DAMPING See Damping.

CRITICAL MASS The minimum mass of fissile material that can sustain a chain reaction under the existing conditions.

CURIE A quantity of radioactive material undergoing $3.7 \times 10^{10}$ (37 billion) transformations (or disintegrations) per second.

DAMPING In this book, a means for dissipating (or absorbing) the vibrational energy of a structure after a disturbance, such as ground motion. Critical damping is the minimum damping that will make a structure become nonoscillatory after a disturbance. Damping ratio is the fraction (or percentage) that the actual damping of a structure is of its critical damping. The larger the damping ratio, the smaller the amplitude of motion when the structure is subjected to a given ground motion. The damping ratios of most structures lie between 0.01 and 0.1 ( 1 and 10 percent), with 0.05 ( 5 percent) as a practical average.

\section{DAMPING RATIO See Damping.}

DESORPTION In general, the freeing or the removal of a substance from its sorbed state; in this book, the removal by relatively uncontaminated water of radioactivity held by rock surfaces (i.e., by sorption). See Sorption.

DISPERSION In this book, the mixing of contaminated water with uncontaminated water at the boundary where they meet. Dispersion results in a decrease in the concentration of radioactivity in the water by dilution.

DISTRIBUTION COEFFICIENT In this book, a measure of the way in which a particular isotope is apportioned between groundwater and the solid rock material in an aquifer.

DOSE In this book, dose or radiation dose implies dose equivalent in rems. See Rem.

DOSE EQUIVALENT A measure of the biological effect of nuclear (or ionizing) radiation absorbed by the body. See Rem.

DOSE RATE See Exposure Rate. 
DOSIMETER An instrument, such as a film badge or a thermoluminescent dosimeter (TLD), for determining accumulated exposure to gamma rays. See Thermoluminescent Dosimeter.

DUST CLOUD Either the main cloud or the base surge cloud formed in an excavation explosion. A main cloud may be formed by the venting of high-pressure gases from the cavity. See Base Surge, Cavity.

ECOLOGY The branch of science concerned with the relationships of the various forms of life in an environment with each other and with the environment. A bioenvironmental safety program with regard to nuclear detonations is concerned with minimizing the disturbance to ecological systems (or ecosystems).

ECOSYSTEM See Ecology.

EFFECTIVE HALF-LIFE See Half-life.

ELECTRON A particle having a very small mass (about $1 / 1800$ the mass of a proton or neutron) and a unit negative charge; it is present in all atoms. See Atom, Proton.

EPICENTER In an earthquake (or aftershock), the point on the earth's surface vertically above the hypocenter, the location on a fault where motion (or slip) has caused the earthquake (or aftershock).

EXPOSURE The total (or accumulated) exposure to gamma radiation over an appreciable period of time, expressed in roentgens (or milliroentgens). See Dosimeter, Roentgen.

EXPOSURE RATE The amount of gamma radiation to which an individual would be exposed per unit time, often expressed in roentgens (or milliroentgens) per hour. The exposure rate is commonly used to indicate the level of radioactivity or radiation intensity at a particular time and place. See Roentgen.

FALLBACK The material that falls back to the ground soon after it has been thrown upward and outward in a cratering explosion. The fallback carries down with it most of the radioactivity produced in a nuclear explosion.

FALLOUT The gradual return of particles to the ground from the clouds (main cloud and base surge) formed in a cratering explosion. Also, the material itself. Local (or early) fallout occurs downwind from the explosion site within about one day after the detonation. Delayed fallout consists of very small particles that ascend to high altitudes and return to earth slowly over a very large area. Fallout sector is the area over which local fallout is 
predicted from wind speeds and directions from the surface to the top of the cloud.

\section{FALLOUT SECTOR See Fallout.}

FAULT A break in the earth's crust and a displacement of rock (in any direction) along the length of the break. A fault may be apparent at the ground surface or it may be hidden (or inferred).

FISSION The splitting of the nucleus of a heavy atom into nuclei of two lighter atoms, with an accompanying decrease in mass. The loss of mass results in the release of a large amount of energy. Fission is usually caused by the absorption of a neutron by the nucleus. The important fissile nuclei are uranium-235 and plutonium-239. Compare Fusion. See Neutron, Nucleus.

FISSION PRODUCTS A general term for the complex mixture of substances produced from nuclear fission. Approximately 80 different primary fission fragments result directly from some 40 different modes of fission. These radioactive fission fragments through decay produce additional (daughter) products; the resulting complex mixture of fission products contains about 200 different isotopes of more than 35 elements. See Fission, Radioactivity.

FLARING In this book, the burning, at the top of a tall pipe or stack, of natural gas released in an underground nuclear explosion the purpose of which has been to stimulate the flow of the gas.

FOCAL DEPTH The vertical distance between the hypocenter and the earth's surface (epicenter) in an earthquake (or aftershock). See Aftershocks, Epicenter, Hypocenter.

FUSION The combining (or fusing) of two very light nuclei to form a nucleus of a heavier atom. There is a net loss of mass which results in the release of a large amount of energy. Deuterium and tritium, the isotopes of hydrogen, are commonly used in this process. Compare Fission. See Isotopes, Nucleus.

GAMMA RAYS Radiations of high energy originating in atomic nuclei and frequently accompanying the emission of alpha and beta particles in radioactive decay. Gamma rays can travel considerable distances through air, although the intensity decreases rapidly at increasing distances from the source. See Radioactivity.

GROUNDWATER A natural underground water system. See Aquifer. 
HABITAT The environment (e.g., desert, seashore, woodland, etc.) in which a particular plant or animal is normally found.

HALF-LIFE The time for the radioactivity (the emission of alpha or beta particles or gamma rays) of a quantity of an isotope to decay to half its original value. The effective half-life of an isotope is the time required for the radioactivity of that isotope in the body (or in an organ) to decrease to half its original value through both decay and biological elimination. The effective half-life is also applied in this book to the radioactivity of iodine in cows' milk. See Isotopes, Radioactivity.

HYPOCENTER The location on a fault where the motion (or slip) responsible for an earthquake (or aftershock) occurs; the focus of an earthquake (or aftershock). See Aftershocks.

INDUCED ACTIVITY In this book, radioactivity that results when certain substances absorb neutrons from a nuclear explosion. See Radioactivity.

ISOTOPES Forms of the same element having identical chemical properties but differing in their atomic masses (owing to different numbers of neutrons but the same numbers of protons in their respective nuclei) and in their nuclear properties (e.g., radioactivity and fission). See Neutron, Nucleus, Proton.

KILOTON One thousand tons; used to express energy released in a nuclear explosion. See Yield.

LINE-OF-SIGHT PIPE A straight pipe leading from a nuclear device through the stemming material for the transmission of radiations produced in the explosion. Line-of-sight pipes are generally used for scientific experiments. See Stemming.

MEGATON One million tons or one thousand kilotons; used to express the energy released in a nuclear explosion. See Yield.

NEUTRON An uncharged (neutral) particle present in all atomic nuclei except those of ordinary (light) hydrogen. Its mass is approximately the same as that of a proton. Neutrons are required to initiate fission, and large numbers of neutrons are produced by both fission and (especially) fusion reactions in nuclear explosions. Absorption of neutrons by some substances leads to the production of radioactive isotopes. See Induced Activity, Nucleus, Proton.

NUCLEUS The small, positively charged core of an atom. It is only about $1 / 10,000$ the diameter of the atom but contains nearly all the atom's mass. All nuclei contain both protons and neutrons, 
except the nucleus of ordinary (light) hydrogen, which consists of a single proton. See Neutron, Proton.

PERIOD OF VIBRATION In this book, the time taken for a structure or the ground to move from one extreme position to the other and back again in vibrations caused by a disturbance.

PICOCURIE One picocurie is a millionth of a millionth part $\left(10^{-12}\right)$ of a curie. It represents a quantity of radioactive material that undergoes 2.2 transformations (or disintegrations) per minute. See Curie.

PLOWSHARE A program of the U.S. Atomic Energy Commission for the research and development of peaceful uses of nuclear explosives. The name comes from the Bible: Isaiah 2:4: ". . and they shall beat their swords into plowshares."

PLUME In this book, a visible column of noncondensable gases escaping from an underground nuclear explosion that is not completely contained. See Contained Explosion.

PROTON A particle, about 1800 times as heavy as an electron, with a unit positive charge. It is physically identical with the nucleus of an ordinary (light) hydrogen atom. All atomic nuclei contain protons. See Electron, Nucleus.

RAD (Acronym for Radiation Absorbed Dose.) The unit of absorbed dose of ionizing radiation. A dose of 1 rad means the absorption of 100 ergs of radiation energy per gram of absorbing material.

RADIATION DOSE See Dose.

RADIATION INTENSITY See Exposure Rate.

RADIATION PROTECTION GUIDE The total radiation dose equivalent received over a certain period of time (e.g., 1 year), in addition to that from natural background radiation and medical and dental $\mathrm{X}$ rays, that should not be exceeded as a result of normal peacetime activities. The guides recommended by the Federal Radiation Council have been used by the U.S. Atomic Energy Commission in formulating standards for radiation protection. See Dose Equivalent, Rem.

RADIOACTIVITY The spontaneous emission of radiation, generally alpha or beta particles, often accompanied by gamma rays, from the nuclei of an (unstable) isotope. As a result of this emission, the radioactive nucleus changes (decays) into a nucleus of an isotope of a different element, which may or may not be radioactive. Ultimately, as a result of decay, a stable (nonradio- 
active) end product is formed. See Alpha Particle, Beta Particle, Gamma Rays, Half-life, Isotopes.

RADIOACTIVITY CONCENTRATION GUIDE The concentration of any specified radioisotope, often expressed as picocuries per unit volume (e.g., 1 milliliter), that should not be exceeded in air and water (or milk) for unrestricted consumption. The radiation dose would be equivalent to the Radiation Protection Guide value. The Federal Radiation Council's guides are the basis of the U. S. Atomic Energy Commission's concentration standards for radioactivity in air and water. See Curie, Picocurie, Radiation Protection Guide.

RADIOISOTOPE A radioactive isotope. See Isotopes, Radioactivity.

RADIOLOGICAL SAFETY PROGRAM In this book, plans and operations designed to assure that the radioactivity from an underground nuclear detonation will not be a hazard.

RAMS (Acronym for Remote Area Monitoring System.) A network of permanent and temporary instrument stations for monitoring gamma-ray exposure rates on the ground within the Nevada Test Site. May also be used in nuclear explosions off the Test Site. RAMS would generally provide the first indication if any radioactivity escaped into the atmosphere.

RAREFACTION WAVE In this book, the wave reflected back into the ground when the shock wave from an underground explosion reaches the surface; a tension wave rather than a pressure wave, for its pressure is less than that of the surrounding medium (whereas the pressure of the shock wave is greater than that of the surrounding medium). See Shock Wave.

REFLECTED WAVE See Rarefaction Wave.

REM [Acronym for Roentgen Equivalent (in) Man.] The unit of dose equivalent of radiation. The dose equivalent (or dose) in rems is a measure of the biological effect of the radiation exposure and is obtained by multiplying the absorbed dose (in rads) by a "quality factor" for the particular radiation (or radiations). A millirem is a one-thousandth part of a rem. See Dose Equivalent, Rad.

RESONANCE In this book, the situation in which the natural vibration period of a structure is equal (or close) to that of a ground motion period. At resonance the response of the 
structure to the ground motion is greatly enhanced, especially if the structure has a small damping ratio. See Damping, Period.

RESPONSE SPECTRUM The response of a series (or spectrum) of simple oscillators, with a postulated damping ratio, subjected mathematically to a particular ground motion. In this book, the response spectrum is plotted as a curve on four-way logarithmic graph paper showing the variations of the peak acceleration, displacement, and velocity of the oscillator with its vibration period. See Damping, Period of Vibration.

ROENTGEN A unit of exposure to gamma (or $\mathrm{X}$ ) radiation. It is that amount of gamma (or $\mathrm{X}$ ) rays required to produce ions carrying 1 electrostatic unit of electrical charge (either positive or negative) in 1 cubic centimeter of dry air under standard conditions. A milliroentgen is a one-thousandth part of a roentgen.

SCALED DEPTH OF BURIAL In this book, the actual depth of burial (in feet) of an underground nuclear device divided by the cube root of the energy yield of the explosion (in kilotons TNT equivalent). It provides a comparison of the effective depths of burial for explosions of different yields. For a contained explosion, the scaled depth of burial must exceed a value appropriate to the yield. See Contained Explosion.

SEEPAGE Slow diffusion to the atmosphere of noncondensable gases from an underground nuclear explosion.

SEICHE An up-and-down surface oscillation in a partially or completely landlocked body of water usually induced by a strong earthquake.

SEISMIC WAVE A wave resembling an earthquake wave and causing only a temporary displacement of the rock medium, the recovery of which is accompanied by ground vibrations.

SEISMOGRAM A record of ground motion or of the vibrations of a structure caused by a disturbance, such as an underground detonation or an earthquake.

SEISMOMETER An instrument used to indicate ground motion caused by an underground detonation (or an earthquake) and the response of structures to ground motion.

SEISMOGRAPH A system for amplifying and recording the signals from seismometers.

SHOCK WAVE A sharp pressure pulse in air, water, or earth, propagated from an explosion. The pressure rises sharply to a 
peak at the shock front, and then decreases fairly rapidly. A shock wave in air usually is called a blast wave. See Blast Wave, Rarefaction Wave.

SORPTION A general term to include the different ways in which a solid can remove dissolved substances from a solution. Specifically, in the movement of water contaminated by radioactivity over rock surfaces, the rock takes up (sorbs) much of the radioactivity. See Desorption.

STABLE CAVITY See Cavity.

STEMMING The plugging of a hole or tunnel, in which a nuclear detonation device has been placed, to prevent the escape of radioactivity into the atmosphere.

STRAIN A measure of the displacement or distortion of a material caused by stress. See Stress.

STRESS A measure of the internal forces generated in a material in resisting changes that tend to be induced by external forces. The stress causes strain in the material. See Strain.

SUBSIDENCE CRATER See Crater.

THERMOLUMINESCENT DOSIMETER (or TLD) A dosimeter containing a chemical that emits light upon heating after exposure to gamma radiation. See Dosimeter.

\section{THROWOUT CRATER See Crater.}

TNT EQUIVALENT See Yield.

TRITIUM A radioactive isotope of hydrogen with a mass of 3 units (two neutrons and one proton in the nucleus) produced, in particular, by the action of neutrons on lithium nuclei. It has a half-life of 12.3 years. All natural waters contain a certain (background) concentration of tritium. See Half-Life, Isotopes, Neutron, Nucleus, Radioactivity.

TSUNAMI A train of traveling waves generated in the ocean by a large-scale submarine disturbance caused by substantial vertical displacement of the sea floor as a result of a strong earthquake. When a tsunami reaches shallow water at a shoreline, the height of the waves can be greatly amplified and serious flooding may occur in appropriate situations.

TUFF Various types of consolidated particulate material of volcanic origin.

VAPORIZATION CAVITY See Cavity.

VENTING In this book, the prompt escape to the atmosphere of gases and solid residues from an underground explosion. 
VIBRATION PERIOD See Period of Vibration.

WIND SHEAR Vertical changes of wind speed and direction over a particular location on the ground.

YIELD The total effective energy released in a nuclear explosion. It is usually expressed in terms of the equivalent tonnage of TNT required to produce the same explosive energy release. See Kiloton, Megaton. 


\section{INDEX}

Aardvark event, 62

Accidental release, radioactivity, 52 , 53

meteorological program, 139, 150

prevention, 55,56

radiological program, 162

Aerial sampling, 165, 166

surveys, 226

tracking, 146, 149, 165, 166, 175, 249

Aftershocks, 121, 122, 255

energy, 122

epicenters, 127-129

and fault displacement, 128, 129

frequency, 126-129

hypocenters, 128,129

Air, radiological surveillance, 171,

176,177

sampling stations, 171

Air Force, U. S., radar, 146

Air Resources Laboratory, 138, 139 , $165,175,216,222,223$

Aircraft tracking, 146, 149, 165, 166, 175,249

Alaska, Department of Fish and Game, 211

University of, 223

Aleutian Islands, 4, 206, 211 see also Amchitka Island earthquakes in, 134,135

tsunami formation, 134, 135

Alluvium, $56 \mathrm{fn}, 61,255$

ground motion, 85,103

seismic effects, 118

Alpha particles, 12, 13, 255

Amargosa Desert springs, 64, 65, 81

Amchitka Island, 2, 4

see also Milrow event

aftershocks, 128, 129

bald eagle on, 205, 208

bioenvironment, 205-208

bioenvironmental studies, 191, 194, $208,209,223$

endangered species, 206

environmental statement, 218

fault displacement, 125

hydrology, 68

peregrine falcon on, 206, 208

sea otters, 206, 210, 211

surface waters, radioactivity, 209

tsunamis, 133,134

vegetation, 200

wave measurement, 134, 222

Amplitude, vibration, $88 \mathrm{fn}, 91,255$

and damping, 92

Animal Investigation Program, 204

Aquifer, 60, 221, 255

see also Groundwater 
flow rate in, $66,67,70$

radioactivity in, $71-81$

Arizona, University of, 223

Atom, 9, 255

structure of, 10

Atomic energy, 18

see also Nuclear energy

number, 11,255

Background radiation, 155, 161, 162, 255

Bandicoot event, 62,63

Base surge, 232, 233, 255

and fallout, 234, 236

Battelle Memorial Institute, 206, 223

Benham event, 111

aftershocks, 126, 128

damage to structures, 111

fault displacement, 124, 125

Beta particle, 13, 256

emission, 17

Bilby event, 62

Bioenvironmental effects, 195-204, 209,210

and earth excavation, 201-203, 251

local, 201-203

postdetonation evaluation, 200, 201

in Milrow event, 209, 210

predictions, 195-201

radiation, 189

shock wave, 191, 195, 201

safety programs, $188-211$

objectives, 188

scope, 189-190

studies, 189-195

at Amchitka Island, 205-210

at Nevada Test Site, 201-204

Biological dose, 156

see also Radiation dose

Biosphere, 256

Biota, 201, 256

Blast wave, 234, 235, 256
Blume, John A., and Associates, 107, 219,220

Boxcar event, aftershocks, 126

fault displacement, 125

ground motion, 103, 104

Bureau of Mines, U. S., 114, 221

Burial, depth of, see Depth of burial

Cabriolet event, 231, 233

Calibration test, 2, 4

California, University of, 204, 213

California Institute of Technology, 130

Cattle, on Nevada Test Site, farm, 179

range, 186

Cavity, 36-40, 43-47, 256

collapse, 39

formation, 36-39, 43

radius, and energy yield, 37,38

calculation, $44-47$

and depth, 46

stable, 37-39

temperature and pressure, 50

vaporization, $36-38$

Central Nevada Supplemental Area, 4 calibration test, 87 see also Faultless event

hydrology, 67, 68, 70

Cesium-137, in chimney, 71

in groundwater, 71,78

half-life, 16

sorption in aquifer, 78

Chimney, 40, 41, 256

dimensions, 48

formation, 40,41

groundwater in, $71,74,75$

Clean explosive, $31,164,235,256$

Cloud, base surge, see Base surge dust, 232, 233

fallout from, 234

see also Fallout 
height, $147,148,244$

main, 232, 233

radiation from, 247,248

tracking, 146, 147, 165, 166, 249

Coast and Geodetic Survey, U. S., see National Ocean Survey

Colorado School of Mines, 130

Concentration guides, radioactivity, 160,161

Contained explosion, 42, 43, 256 radiological safety, 137, 138, 150, $155,162,215,216$

Containment Evaluation Panel, 216

Cosmic rays, $155,161,162,256$

Council on Environmental Quality, 218

Cows' fodder, analysis, 174 radioiodine in, 178,181

Cracking radius, 47,48

Crater, 40, 41, 228-231, 257 see also Excavation

apparent, 229

subsidence, 40,41

throwout, 228

and depth of burial, 229-231

and dust clouds, 232,233

formation, 228-231

lip, 229

Critical damping, 93, 257

Critical mass, 23, 257

Curie, 160, 257

Damage, see Structures

Damping, 92, 257

critical, 93, 257

ratio, 93,257

and structural response, 92, 93, 95

Dams, instrumentation, 88 safety of, 113, 114

Danny Boy event, 231, 233

Daughter nucleus, 16, 17

Defense Atomic Support Agency, $214,216,222,224$
Defense Nuclear Agency, see Defense Atomic Support Agency

Delco Electronics Division, General

Motors Corp., 222

Depth of burial, $54-56$

and crater formation, 229-231

scaled, 54, 55, 263

minimum for containment, 55

and radioactivity in fallout, 229231

Desorption, 73, 75, 78, 257

Dispersion, in water, 73,257

effect of , 76, 77

Displacement, peak, 99, 100

Distribution coefficients, 75,257

and sorption, 75

Dose, 257

see also Radiation dose

Dose equivalent $156,157,257$

Dosimeter, 167, 258

film badge, 167

stations, 167,168

thermoluminescent (or TLD), 167 , 264

Dust cloud, see Cloud

Earth excavation, see Excavation

Earthquake Mechanism Laboratory, 130,222

Earthquake terminology, 121, 123

Earthquakes, 121

see also Aftershocks

and seiches, 134

and tsunamis, 131, 132

and underground detonations, 121 , $122,130,131$

Ecological systems, aquatic, 193-195

description of, 191-195

effects on, 189, 191, 201

see also Bioenvironmental effects

freshwater, 193, 194, 197

marine, 194, 195, 197

terrestrial, 192, 193, 197 
Ecology, 258

Ecosystems, see Ecological systems

Effective half-life, 163, 260 iodine-131, in milk, 181 tritium, 164

Effects Evaluation Report, 216, 217

Elastic deformation, 36 wave, 36,83 see also Seismic wave

Electron, 10, 258 see also Beta particle

Elements, 9

Endangered species, 193, 205

Environmental effects, see Bioenvironmental effects

Environmental Protection Agency, $165,171,185,216,223,226$ see also Southwestern Radiological Health Laboratory veterinarian, 185, 186

Environmental Research Corporation, $103,105,219$

Environmental statements, 218 surveillance, radiological, see Radiological surveillance

Epicenter, 123, 258 of aftershocks, 127-129

Equation of state, 44, 45, 47

Excavation, 228 see also Crater, throwout base surge, $232-236$ bioenvironmental effects, 201-204 program, $188,190,192$

blast wave, 234,235 dust clouds, 146, 232-238 tracking, 146-149, 249

explosives for, 31,235 and fallout, 234-247 see also Fallout protective action, 249 radiation predictions, 235-249 and food chains, 196 mechanism, 228-231
Explosion, 18, 23

fission, 23, 24

fusion, 29-31

nuclear, 18-32 yield of, 20

Explosive, 18 see also Explosion clean, see Clean explosive

Exposure, radiation, 155-157, $167 \mathrm{fn}, 258$ see also Radiation exposure rate, $167 \mathrm{fn}, 258$

Extensometer, 116

Fallback, 229, 232, 258

Fallout, 31, 139, 258, 234, 258 delayed (or worldwide), 204, 234 , 249,258

early (or local), 234, 235, 258

models, 223, 244-246

and precipitation, 145, 245, 246

radiation exposure, $246-248$ intensity, 241-246

radioactivity in, $234,239-241$ sector, 150, 152, 236-239, 258

Faultless event, 87, 89 aftershocks, 126 fault displacements, 123

Faults, 61, 259 concealed, 61,62 and detonation point, 56-58 displacements, 123-126 and aftershocks, 128 observable, 61 and surface fractures, 62 at Yucca Flat, 61

Federal Aviation Administration radar, 145, 146

Federal Radiation Council (FRC), 157, 159-161, 181

Film badge, 167

Fish and Wildlife Service, U. S., 205 
Fission, 21-28, 259

chain, 22,23

energy from, 21

explosion, 23,24

fragments, 21, 25

products, $24-28,51,239,246$, 259

see also Fallout

in animals, 204

in flared gas, see Flaring

in groundwater, see Groundwater

radiation exposure, 246,247

radioactive decay, $26-28,71$

release, accidental, 52,53

Flaring, gas, 140, 151, 162, 163, 259

bioenvironmental program, 190

radiological program, 190

Focal depth, 123, 259

of aftershocks, 128, 129

for tsunami formation, 132

Focus, earthquake, see Hypocenter

Food chains, 193-198

Fundamental mode, vibration, 92, 93

Fusion, 29-31, 259

energy from, 29

explosion, 30

radioactivity from, 30,31

Gamma rays, 13-14, 247, 259

Gas acceleration, 229-231, 233

Gas plume, 150

Gas wells, see Wells

Gasbuggy event, 48, 151

damage to structures, 111

ground motion, 105

radioactivity in gas, 164

Geological Survey, U. S., 59, 64, 126, $127,130,216,217,221,222,251$

Geology, and ground motion, 85 and hydrology, studies, 59-70 and underground detonations, $60-63$
Gnome event, 39

cavity, 39, 40

Ground motion, 82-88, 94, 95

see also Response spectrum

contractor, 103, 105, 219

factors affecting, 84, 85, 119

human perception, 83

measurement, see Seismograph

prediction, 103

see also Response spectrum

sensitivity to, 83

structural response to, see

Structural response

studies, 117

Groundwater, 59, 259

see also Aquifer

cesium-137 in, 71, 78

flow rate, $66,67,70$

radioactivity in, $60,70-81$

decay, 77,80

dispersion, 73, 76, 77

migration, 71, 72, 78-81

sorption and desorption, 73,75

strontium-90 in, $77-79,81$

tritium in, 77, 79-81

Gun-assembly device, 24

Habitat, 193, 199, 203, 260

aquatic, 204

Half-life, 15, 16, 26, 27, 260

effective, 163,260

iodine-131, in milk, 181

tritium, 163, 164

Handley event, 111

aftershocks, 128

damage to structures, 111

fault displacements, 125

Harvey Gap Dam, 113, 114

Hawaii, University of, 222

Haymaker event, 62

Heat of vaporization, 44

High-rise structures, 82

and ground acceleration, 103, 108 
and ground motion, 96

analysis, 101, 102

effect of distance, 106, 107

effect of yield, 106, 107

instrumentation, 88

vibration, $88,90,92,93$

period, 92

Hoover Dam, 88, 134, 135

Hot Creek Valley, 2-4 see also Faultless event hydrology, 67, 68, 70

Hot spots, 145, 245

Hydrology studies, 59, 60, 63-70 objectives, 63,64

regional, $64-68$

site, 68,69

special, 69,70

Hypocenter, 123, 260

of aftershocks, 128

Implosion device, 24

Induced activity, $28,30,260$

in fallout, 235, 236, 239

in fission explosion, 26-29

in fusion explosion, 30,31

International Commission on Radiological Protection, 157

Iodine, radioactive, in air, 171

escape of, 162,163

in fission products, 51

in milk, 163, 178-181

pathways to man, 178

in thyroid, 159, 160

Iodine-131, in fallout, 249

half-life, 16

effective, in milk, 181

in milk, 173, 181

concentration guide, 161

in thyroid, 174

Isotopes, 11, 260

radioactive, see Radioisotopes

Jorum event, aftershocks, 128

fault displacement, 125
Krypton, radioactive, fission product, 51

escape of, 162, 163

Krypton-85, analysis, 176

in flaring, 140, 151, 163, 176

half-life, 16

Lake Mead, 134, 135, $251 \mathrm{fn}$

Lapse rate, 148

Las Vegas, high-rise buildings, 82, 107 see also High-rise structures strengthening, 110 monitored structures, 90,118 response spectrum at, 100

Lawrence Radiation Laboratory, Livermore, 213, 216, 217, 222, 224

Limited test ban treaty, v, 1, 52

Line-of-sight pipe, 52, 53, 260

Long Shot event, 129, 206, 209

Los Alamos Scientific Laboratory, $213,216,217,222,224$

Mead Lake, see Lake Mead

Medical investigation, 183-185

Medical Liaison Officer Network

(MLON), 183-185

Meteorological program, 137-153, 222

see also Weather

after detonation, 139-40

and detonation time, 139

final, 139

for flaring, 140, 176

measurements, 140-149

and radiation predictions, $149-151$

Milk, iodine in, $161,163,173,178-$

181

after Pin Stripe event, 181

protection of, $181-183$

radioisotopes in, 180

radiological surveillance, 171,176 , 249

sampling stations, $171-173$ 
Milrow event, 125

aftershocks, 128, 129

bioenvironmental effects, 209,210

studies for, $208-210$

and fault displacement, 125

and wave formation, 134

Mines, inspection, 114-116, 221

Modes, vibration, see Vibration period

Nash event, 53

National Center for Earthquake Research, 126, 130

National Council on Radiation Protection, 157, 159, 160

National Environmental Policy Act, 189, 218

National Marine Fisheries Service, 223

National Meteorological Center, 140,143

National Ocean Survey, 87, 126, 219 , 220,222

National Oceanic and Atmospheric Administration (NOAA), see Air Resources Laboratory, Earthquake Mechanism Laboratory, National Ocean Survey

National Weather Service, 138

Neutron, 10, 260

and beta decay, 17

in fission, 21

from fusion, 29,30

induced radioactivity, see Induced activity

Nevada, University of, 130, 221-223

Nevada Applied Ecology Group, 217

Nevada Test Site, 1, 3

animal investigation program, 204

bioenvironmental studies, 201 204

cattle, dairy, 179

range, 186,204

dairy farm, 179 ecology, 201-204

effects evaluation, 216

excavation tests, 251

faults on, 56

geology of, 60-65

hydrology of, $65-67,70$

map of, 3

meteorological measurements, $140-149$

Planning Board, 218

radioactivity, escape, 53

radiological safety program, 164174

structures, test, 90, 97, 118

weather briefings, $151,152,225$

pattern, 138

predictions, $143,148,152$

Noncondensable gases, 51, 71, 150

Nuclear energy, 18

release, fission, $21-24$

see also Fusion

fusion, 29-31

see also Fusion

Nuclear excavation, see Excavation

fission, see Fission

fusion, see Fusion

Nuclear Safety Groups, 215

Nuclear Systems Safety, 215, 216

Nucleus, atomic, 10, 260

daughter, 16, 17

parent, 17

Ohio State University, 223

Oil wells, see Wells

Operational Safety Plan, 224

Pahute Mesa, 3, 124, 125

seismograph stations, 126

Panel of consultants, 218

Parent nucleus, 17

Pasteurized Milk Network, 171, 173 
Period, vibration, see Vibration period

Pibals, 143, 144

Picocurie, 160, 261

Pin Stripe event, 181-183

Plastic deformation, 35, 36

Plowshare program, 261 see also Excavation and commercial products, 164 bioenvironmental studies, 191 effects, 203 effects evaluation, 217 environmental statement, 218 explosive, selection, 31,32 see also Clean explosive geology and hydrology, 59 meteorology for, 138-140, 150, $151,153,176$

objectives, vi radioactivity surveillance, 176 radiological program, 175-177 safety planning, 229 stemming, 33 tests, 1

Plume, gas, 150, 261

Plutonium-239, in explosive, 16 fission, $21-26$

Potassium-40, in body, 161 in soil, 161

Precipitation, and fallout, 145, 146, 246

Protective Action Guide, 181

Proton, 10, 261

Public Health Service, 154, 162, 165, see also Environmental Protection Agency

Quality factor, radiation, 156

Rad, 156, 261

Radiation, and animals, 199 background, $155,161,162$ bioenvironmental program, 189

briefings, 151-153, 225

dose, absorbed, 156

biological, 156

equivalent, $156,157,257$

exposure, $155-157$

from cloud, 155-157

external, 14, 160 measurement, see Dosimeter from fallout, $246-248$

from gas plume, 150

internal, 14, 160 measurement, 174 predictions, $149-151,235-249$ rate, $167 \mathrm{fn}$ injury, investigation, 183-186 intensity, $167 \mathrm{fn}$ monitoring, 149, 165, 175, 226 environmental, 169-174, 176, 177

on ground, 167-169

stations, 169

and plants, 199, 203

protection, guidelines, 157-161 standards, 157-161

Protection Guides, 157, 159-161, 261

safety plans, 222,223

see also Radiological safety program

units, 156,157

Radioactive cloud, see Cloud decay, $12-18$ of fission products, $26-28$

products of, $16-18$

rate of, 14-16 see also Half-life isotopes, see Radioisotopes residues, 5,137 see also Cloud, Fallout, Plume

Radioactivity, 12-18, 261 see also Radioactive decay accidental release, see Accidental release 
background, 161

in the body, 161

Concentration Guides, 160, 161, 262

in excavation, 239-241

in groundwater, see Groundwater

in rock melt, 39, 60, 71

Radioiodine program, $156,177-180$ see also Iodine

Radioisotopes, 12

see also Radioactivity

in food chains, 193-198

half-life, see Half-life

in milk, 180

see also Milk

pathways to man, 195-199

model of, 196-198

in water systems, 194

see also Groundwater

Radiological safety program, 154

$187,249,262$

meteorological support, 137-153

see also Meteorological program

for Nevada Test Site, 164-174

for other sites, $175-177$

purpose of, 154

surveillance programs, 169

air, 171, 176, 177

milk, 171, 176, 249

water, 171, 176, 249

RAMS (Remote Area Monitoring

System), 165, 167, 175, 262

Rarefaction wave, 42, 43, 228, 229 , 262

Reflected (shock) wave, 42, 43, 228 , 229,262

Rem, 156, 262

Resonance, 94, 262

see also Seiche

and structural response, 94-97

Response spectrum, 263

determination, $98-101$

and distance from explosion, 106,

107 prediction, 101, 105-107

and structures, 106, 107

and yield of explosion, 106, 107

Reynolds Electrical and Engineering

Co., Inc., 216

Roentgen, 156, 157, 263

Rulison event, 68,151

damage, 111,112

dams, safety of, 113, 114

geology of site, 68

radioactivity in gas, 164

radiological safety plans, 176

safety measures, 220

seismic instrumentation, 88

Safety controls, 213-226

management, 223-226

programs, 9,10

see also Meteorological program,

Radiological safety program

Salmon event, 39

calculations, 49

cavity, 39

damage to structures, 111

- Sandia Laboratories, 213, 216, 217 , 224

Scaled depth of burial, see Depth of burial

Scanner, 174

Scientific Management Centers, 217

Sea otters, 205, 210, 211

Sedan event, 231

crater, 231,232

dust clouds, 231, 233

Seepage, radioactivity, 52-56, 263, prevention, 55, 56

Seiche, 134, 135, 263

Seismic Evaluation Subcommittee, 218

Seismic wave, energy, 36 and aftershock energy, 122, 129 formation, 36,83 reflection and refraction, 84,85 
Seismogram, 87-89, 263

Seismograph, 86, 87, 263 systems, 87,88

Seismometer, 86, 87, 263 on structures, 88

Shock front, 35 wave, $5,228,263$

in air, 234, 235 see also Blast wave formation, 34 propagation, $35,36,46,47$ reflection, 42, 43, 228, 229, 262

Smithsonian Institution, 223

Southwestern Radiological Health Laboratory, 165, 166, 171, 174, $175,178-181,183-185,204$, $223,224,226$

Sorption, 73, 75, 77-79, 264 and distribution coefficient, 75

Spalling, 43, 228, 229

Standards, radiation protection, $155-161$

Stemming, 33, 34, 264

platform, 34

safety plans, 214-216

Strain, 47, 264

earth, measurement, 130

meter, 130

in rock, 47

Stress, 47, 264

in rock, 47

and damage to structures, 86

Strontium-90, in groundwater, 71 , $77-79,81$

distribution coefficient, 78 migration in, 78,79

sorption and desorption, 78

half-life, 16

Structural response, $86,88,95-102$, 105-112 see also Response spectrum contractor, 107, 219, 220 and damping, 95 to ground motion, $83,96-102$ measurement, 85

and resonance, 94--96

studies of, 96-102

variations, 86

Structures, damage, experience, 111 , 112

prediction, 107-112

probable, 109, 110

survey, 108, 109

vibration, 90-96

modes, 92,93

period, $91,92,94-96$

measurement, 97

and resonance, 94-97

Subcritical mass, 23

Submarine earthquakes, and

tsunamis, 131,132

Supercritical mass, 23,24

Surface water, 65

radioisotopes in, 250,251

Tamarin Committee, 222

Teledyne Isotopes, Inc., 71, 221

Temperature, air, 148

Tennessee, University of, 223

Test Manager, 224-226

advisory panel, 224-226

Tetra Tech, Inc., 222

Tetroons, 146, 147

Thermoluminescent dosimeter (TLD), 167,264

Thermonuclear device, 30 see also Fusion

Thyroid, dose from Pin Stripe event, 183

iodine in, $158,159,160,174,177$, 178

protection standard, 158,159

Time-history response method, 101, 102,117

Tonopah, ground motion, 119

Tritiated water, 29, 51, 52, 72 see also Tritium 
in body, 164

in flared gas, $140,151,163$

in gas plume, 150

Tritium, 11

see also Tritiated water

analysis for, in body, 174

in flaring, 163

in the body, 163, 164

in fission, 32

in flared gas, 163,176

in fusion explosions, $29-32$

in groundwater, see Tritiated water in natural gas, 164

in nuclear explosions, $30-32$

in water, concentration guide, 161 , 164

on Amchitka Island, 209

Tsunami, 131-134, 264

causes, 132

characteristics, 132

formation, 131,132

research on, 134, 222

and underground detonations, 133 , 134

Tuff, $56 \mathrm{fn}, 61,264$

Underground testing, objectives, v, vi

Uranium-235, 12

fission, $21-26$

Uranium-238, 12

Urine, analysis for tritium, 174, 177

Utah, University of, 130, 222, 223

Utah State University, 223

Venting, of radioactivity, 52, 56-58, 264

Veterinary investigations, 185, 186

Vibration period, $88 \mathrm{fn}, 91,92,94-$

96, 261

and damping, 92,93

measurement, 97

modes, 92,93 and resonance, see Resonance

and response spectrum, 98-101

see also Response spectrum

of structures, see Structures

Washington, University of, 223

Water, sampling stations, 171

radiological surveillance, 171,176 , 249

supplies, safety of, 70-81

absence of radioactivity, 70,71 ,

221

surface, see Surface water

tritiated, see Tritiated water

underground, see Groundwater

wave effects, $131-135$

see also Seiche, Tsunami

Weather, briefings, $139,151,152$, 225

distribution of, 145

predictions, 222, 223

program, see Meteorological program

stations, 140, 141

Wells, inspection, 114, 116, 221

Whole-body counter, 174

Wind, and fallout sector, 236-239

measurements, 140-145

predictions, 143,145

shear, 142, 265

vector, weighted, 237

Xenon, $51,71,162,163$

Yield, 20, 265

Yucca Flat, 2, 78, 81

geology, 60-63

hydrology, $64-67$

temperature, 148

weather pattern, 138

winds, 142 
Printed in the United States of America USAEC Division of Techmcal Information Extension, Oak Ridge, Tennessee 